\title{
ELECTRON-PHONON INTERACTIONS
}

\section{IN LOW DIMENSIONAL STRUCTURES}

\author{
David Romwald Leadley
}

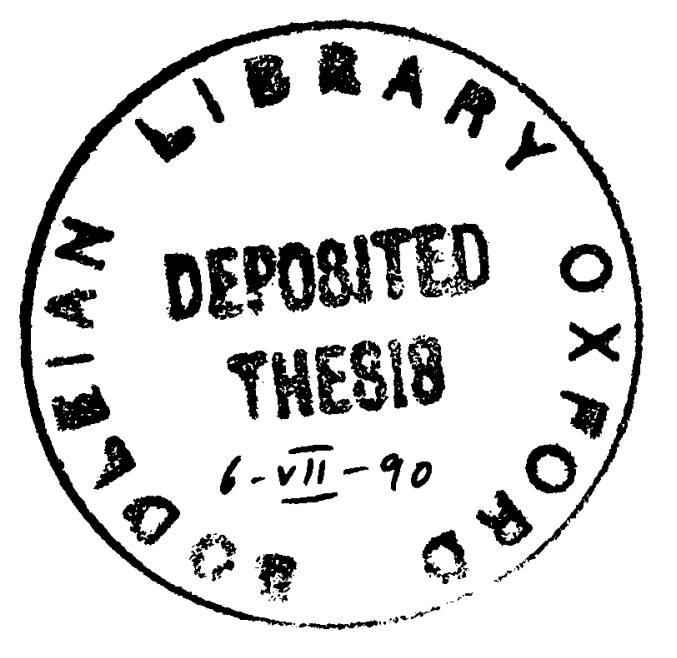

A thesis submitted for the degree of

Doctor of Philosophy

University College,

Oxford

Trinity Term, 1989 


\title{
ELECTRON-PHONON INTERACTIONS IN LOW DIMENSIONAL STRUCTURES
}

\author{
By David R. Leadley
}

A thesis submitted for the degree of Doctor of Philosophy University College, Oxford.

Trinity 1989

\section{ABSTRACT}

Transport properties of the two-dimensional electron gas (2DEG) in high magnetic fields are used to investigate scattering processes affecting the resistivity of GaAs-GaAlAs and GaInAs-InP heterojunctions and quantum wells: especially coupling of electrons to acoustic and optic phonons; and transitions between electric subbands.

The experiments fall into two groups:

A systematic study of magnetophonon resonance (MPR) between 30K and 300K. Resonance positions indicate a coupling substantially below the LO phonon energy, expected from 3D measurements. GaAs-GaAlAs heterojunctions show amplitudes varying smoothly with electron density $\left(n_{s}\right)$ and closely related to the $4 \mathrm{~K}$ mobility. On rotation in magnetic field they decrease rapidly as the resonance position returns to the LO value. In modulation doped structures the damping factor is determined by remote impurity scattering. As $n_{s}$ is increased in GaInAs-InP the coupling frequency decreases dramatically from the GaAs-like $\mathrm{LO}$ at $272 \mathrm{~cm}^{-1}$ to the InAs-like TO at $226 \mathrm{~cm}^{-1}$. At higher electric fields the 'normal' MPR maxima invert, starting at low magnetic fields, to form 'hot electron' MPR minima, with maximum amplitude at $\sim 60 \mathrm{~K}$. This is the first direct observation of HEMPR in $2 \mathrm{D}$ and is explained in a diffusion picture. At lower electric fields, additional resonances are identified with resonant cooling by inter-subband scattering. Comparisons are made with calculations and explanations sought including consideration of interface phonons; coupled plasmon-phonon modes; and shifts of the resonance positions due to the shape of the density of states.

Low temperature magnetoresistance measurements in GaAs-GaAlAs heterojunctions with more than one occupied electric subband. Shubnikov-de Haas oscillations in perpendicular magnetic fields contain non-additive terms at electron temperatures $\gtrsim 2 \mathrm{~K}$, where acoustic phonon mediated inter-subband scattering is comparable to intra-subband scattering. Subband separations and greatly enhanced $\mathrm{g}$-factors [largest for electrons in the upper subband] are deduced from the oscillations. Damping of the oscillations in field, gives values for quantum lifetimes $\left(\tau_{s}\right)$, much smaller than $\tau_{t}$, deduced from mobility. With two subbands occupied $\tau_{s}$ is always largest for the upper subband, while relative sizes of $\tau_{t}$ depend on sample quality. Study of electron energy loss rates, from thermal damping of the oscillations, shows enhancement in the region $k T_{e} \sim \hbar \omega_{c}$, which is evidence for cyclotron phonon emission. Depopulating subbands in parallel fields causes the resistance to drop, by up to $60 \%$, due to suppression of inter-subband scattering. Systematic studies show this scattering rate is independent of $n_{s}$. 


\section{Acknowledgements}

I wish to express my gratitude to all those who have made the work of this thesis both possible and enjoyable. I would especially like to thank:-

Professors E.W.J. Mitchell, W. Hayes, P.G.H. Sanders and R.A. Cowley for extending me the facilities of the Clarendon Laboratory.

My supervisor Dr. Robin Nicholas for invaluable encouragement and guidance, his continual enthusiasm and for many stimulating discussions.

Drs J.J. Harris and C.T. Foxon of the Philips Research Laboratories, Redhill and Drs. M.S. Skolnick, S.J. Bass and L.L. Taylor from the Royal Signals and Radar Establishment, Malvern for supplying me with many world class samples without which none of this work could have been performed, and Prof. J.C. Portal for allowing me the use of some of his material grown by Mdme. M. Razighi at Thomson C.S.F.

My research colleagues: Mark Brummell for starting me off in the right direction; Debra Barnes and Margaret Hopkins for GaAs-GaAlAs cyclotron resonance measurements; Didier Gauthier for close collaboration on the magnetophonon effect in GaInAs-InP; Alan Usher for creating and taming the ${ }^{3} \mathrm{He}$ system; Dave Howell for many length discussions; and Niel Bassom, Stephanie Hayward, Jenny Mallet, Rob Martin, Rick Martinez, Firoz Nasir, Nick Pulsford, John Singleton, Andrew Suckling, Ganesh Sundaram, Martin Tatham, Richard Warburton, Mark Watts and Graham Wiggins for generally improving the quality of life in the Clarendon.

Drs. P. Warmenbol, F.M. Peeters and Prof. J.T. Devreese of the University of Antwerp for helpful discussions and calculations of the magnetophonon effect.

The staff of the Clarendon Laboratory, especially Harry Jones, Martin Whitworth and Jack Day for provision (and maintainance!) of the high magnetic field facility.

Finally I must thank those who supported me financially: the Science and Engineering Research Council and G.E.C. Hirst Research Centre for providing my CASE Studentship, and Dr Mike Kelly my industrial supervisor;

Philips Research Laboratories, Redhill for funding my Junior Research Fellowship at University College;

The Dee Corporation and St. Hugh's College for providing a most generous Senior Scholarship, and Dr. Gillian Gehring for extending support and a warm welcome;

Last, but far from least, Christ Church for providing a Dixon Scholarship and five memorable and enjoyable years of my life. 


\section{CONTENTS}

Abstract vi

Acknowledgements $\quad$ iv

Contents iii

CHAPTER 1: Electronic States in Semiconductor Heterostructures

1.1 Introduction 2

1.2 Semiconductor Heterostructures 3

1.3 Quantisation in Heterostructures 9

$1.4 \quad$ Additional Couplings Affecting the Band Structure 15

1.5 Densities of States in 2-D 19

CHAPTER 2: Magneto-transport Measurements and Sample Characteristics

$\begin{array}{lll}2.1 & \text { Introduction } & 25\end{array}$

2.2 Magneto-transport 25

2.3 The Magnetophonon Effect 34

$2.4 \quad$ Cyclotron Resonance 39

2.5 The Magnets 43

2.6 Cryogenics 44

2.7 Electrical Measurements 45

$2.8 \quad$ Sample Parameters 51

CHAPTER 3: Magnetophonon Resonance in Two-dimensional GaAs-GaAlAs Structures

$\begin{array}{lll}3.1 & \text { Introduction } & 60\end{array}$

3.2 Damping of the Oscillations 64

3.3 Magnetophonon Resonance Amplitudes 70

3.4 Magnetophonon Effective Mass $\quad 88$

3.5 Resonance Positions and Phonon Frequencies 93

3.6 Magnetophonon Oscillations in the Hall Coefficient 102

$\begin{array}{lll}3.7 & \text { Rotation in Magnetic Field } & 103\end{array}$

$\begin{array}{lll}3.8 & \text { Resonances in GaAs-GaAlAs Quantum Wells } & 107\end{array}$

$\begin{array}{lll}3.9 & \text { Discussion of Possible Explanations } & 111\end{array}$

$\begin{array}{lll}3.10 & \text { Summary } & 118\end{array}$ 
CHAPTER 4: Magnetophonon Resonance in GaInAs-InP Heterostructures

$\begin{array}{lll}4.1 & \text { Introduction } & 120\end{array}$

4.2 Optic Phonon Modes in GaInAs-InP 121

$\begin{array}{lll}4.3 & \text { The Resonance Position Varies with Carrier Density } & 124\end{array}$

$\begin{array}{lll}4.4 & \text { Rotation in a Magnetic Field } & 130\end{array}$

4.5 Resonance Amplitudes and Damping Factors 132

4.6 Discussion of the Resonance Postitions 136

$\begin{array}{lll}4.7 & \text { Summary } & 141\end{array}$

CHAPTER 5: Hot Electron Magnetophonon Resonance in Two Dimensions

$\begin{array}{lll}5.1 & \text { Introduction } & 143\end{array}$

$\begin{array}{lll}5.2 & \text { Hot Electron Energy Loss Mechanisms } & 144\end{array}$

$\begin{array}{ll}\text { 5.3 Hot Electron Magnetophonon Resonance in GaAs-GaAlAs } & 146\end{array}$

$\begin{array}{lll}5.4 & \text { Comparison with Theory } & 152\end{array}$

5.6 Energy Loss by Resonant Inter-subband Scattering 160

$\begin{array}{lll}5.7 & \text { Summary } & 163\end{array}$

CHAPTER 6: Inter-subband Scattering in GaAs-GaAlAs Heterojunctions

$\begin{array}{lll}6.1 & \text { Introduction } & 165\end{array}$

6.2 Occupation of Higher Electric Subbands 166

6.3 Magnetoresistance Measurements in Perpendicular Fields 172

$\begin{array}{lll}6.4 & \text { Effective } \mathrm{g} \text {-Factors } & 178\end{array}$

6.5 Magnetoresistance Measurements in Parallel Magnetic Fields 184

6.6 Effects of Inter-Subband Scattering on Shubnikov-deHaas Oscillations 191

$\begin{array}{lll}6.7 & \text { Summary } & 199\end{array}$

CHAPTER 7: Quantum and Classical Relaxation Times - Their Relation to Scattering Mechansims in Multisubband GaAs-AlGaAs Heterojunctions

$\begin{array}{lll}7.1 & \text { Introduction to } \tau_{s} \text { and } \tau_{\mathfrak{t}} & 201\end{array}$

7.2 Measurement of the Quantum Lifetime 202

7.3 Upper Subband Transport Mobility 208

7.4 Other Sources of Low Field Magnetoresistance 212

$\begin{array}{lll}7.5 & \text { Summary } & 216\end{array}$ 
CHAPTER 8: Cyclotron Phonon Emission and Electron Energy Loss Rates in GaAs-GaAlAs Heterojunctions

$\begin{array}{lll}8.1 & \text { Introduction } & 218\end{array}$

$\begin{array}{lll}8.2 & \text { Measurement of Electron Temperature } & 220\end{array}$

8.3 Enhancement of Loss Rate in Small Magnetic Fields 226

$\begin{array}{lll}8.4 & \text { Cyclotron Phonon Emission } & 229\end{array}$

8.5 Intra-Landau Level Energy Loss 231

$\begin{array}{lll}8.6 & \text { Summary } & 233\end{array}$

References 
CHAPTER 1

ELECTRONIC STATES IN SEMICONDUCTOR HETEROSTRUCTURES

1.1 Introduction

2

1.2 Semiconductor Heterostructures

1.2.1 Heterojuntions 3

1.2.2 Modulation Doping 5

1.2.3 Quantum Wells 5

1.2.4 Growth Techniques 6

1.2.5 Control of Electron Density 8

1.3 Quantisation in Heterostructures

1.3.1 Electrical Quantisation - Subbands 9

1.3.2 Quantisation in Magnetic Fields 12

1.4 Additional Couplings Affecting the Band Structure

1.4.1 Band Non-parabolicity

1.4.2 Polaron Coupling

1.5 Densities of States in 2-D

1.5.1 Broadening Mechanisms 19

1.5.2 Localised or Extended? 20

1.5.3 The Shape of the Landau Levels 21 


\section{$1.1 \quad$ INTRODUCTION}

This thesis is concerned with the electronic transport properties of the two-dimensional electron gas (2DEG) found in III-V semiconductors. The experiments are performed in high magnetic fields, at temperatures from $500 \mathrm{mK}$ up to room temperature, and enable deductions to be made about the scattering mechanisms dominating the transport properties under various sets of conditions.

First it is necessary to discuss how the electrons are confined to move in only two dimensions and investigate the resulting distribution of electron energy states. There are naturally occuring 2-D systems, but advances in crystal growth technology have allowed a rapid expansion in the study of specially fabricated structures. In these structures there is much more control over the position of the electrons and so it is possible to achieve very high electron mobilities (Harris et al. 1986). The electronic properties of two dimensional systems were extensively reviewed by Ando, Fowler \& Stern (1982).

One example of a naturally occuring two-dimensional system is provided by electrons bound to the surface of liquid helium. An electron close to the helium surface experiences a force due to the image potential induced by the differing dielectric constants in the gas and the liquid. This force is attractive for electrons outside the helium, but becomes strongly repulsive once inside the liquid, thus binding the electrons to the surface. This system has been studied extensively and is reviewed by Grimes (1978). Another naturally occuring 2DEG is found in the layer semiconductor InSe, first reported by Portal et al. (1980) and discussed more recently by Howell (1989).

In semiconductors, two-dimensional electron systems were first studied in silicon metal-oxide-semiconductor (MOS) devices, where electrons are confined to an inversion layer parallel to the surface, by an electrric field applied to the gate (Fowler et al. 1966). It is also possible to form a 2DEG from charges of the same sign to those found in the bulk of the material in an accumulation layer. The early work on $\mathrm{Si}$ has been extensively reviewed by Ando et al. (1982).

Of interest here are structures with $2 \mathrm{DEG}$ localised at the interfaces between III-V semiconductor alloys (Störmer et al. 1979), and it is to these that we now turn. 


\subsection{SEMICONDUCTOR HETEROSTRUCTURES}

\subsubsection{Heterojuntions}

A heterojunction is an interface between two semiconductors which, in general, have different band gaps and may have different lattice constants. Here we are only concerned with interfaces between lattice matched alloys - for $\mathrm{Ga}_{0.7} \mathrm{Al}_{0.3} \mathrm{As}$ grown on $\mathrm{GaAs}$ the lattice mismatch is $<0.1 \%$ and is only slightly larger for $\mathrm{Ga}_{0.47} \mathrm{In}_{0.53} \mathrm{As}-\mathrm{InP}$. There is currently also much intrest in the properties of strained layers eg. Si-Ge and GaAs-InAs but we will not discuss them here.

When an interface is formed between alloys of different band gap, eg. GaAs and GaAlAs as shown in Fig. 1.1a, electrons will flow from the material of higher conduction band energy to that of lower conduction band energy until the Fermi energies at the interface are equalised, and the Fermi levels at a long distance correspond to charge neutrality in each material. The movement of electrons creates a depletion region of positive space charge in the wide gap material (in the example of Fig. 1.1 - GaAlAs) and an accumlation of electrons in the narrow gap material (GaAs). The resulting potential distribution appears as a bending of the bands which, together with the conduction band discontinuity, forms a potential well in the narrow gap material, as shown in Fig. $1.1 \mathrm{~b}$. This potential well is roughly triangular in shape and, if sufficiently narrow, will give rise to widely spaced quantised electron energies (in the $z$ direction), called subbands, and constrain the electrons to move parallel to the interface (in the $x y$ plane). In p-type material the above mechanism gives rise to holes localised in a potential well in the valence band of the narrow gap material. It should be noted that this thesis is exclusively concerned with Type 1 heterostructures, in which the conduction band of the larger band gap material is higher in energy and the valence band lower in energy than those of the narrower gap material, which means both electrons and holes will be localised in the narrow gap material as opposed to Type II material where 2D hole gases may occur on the opposite side of the interface to the 2DEG. 
(a)

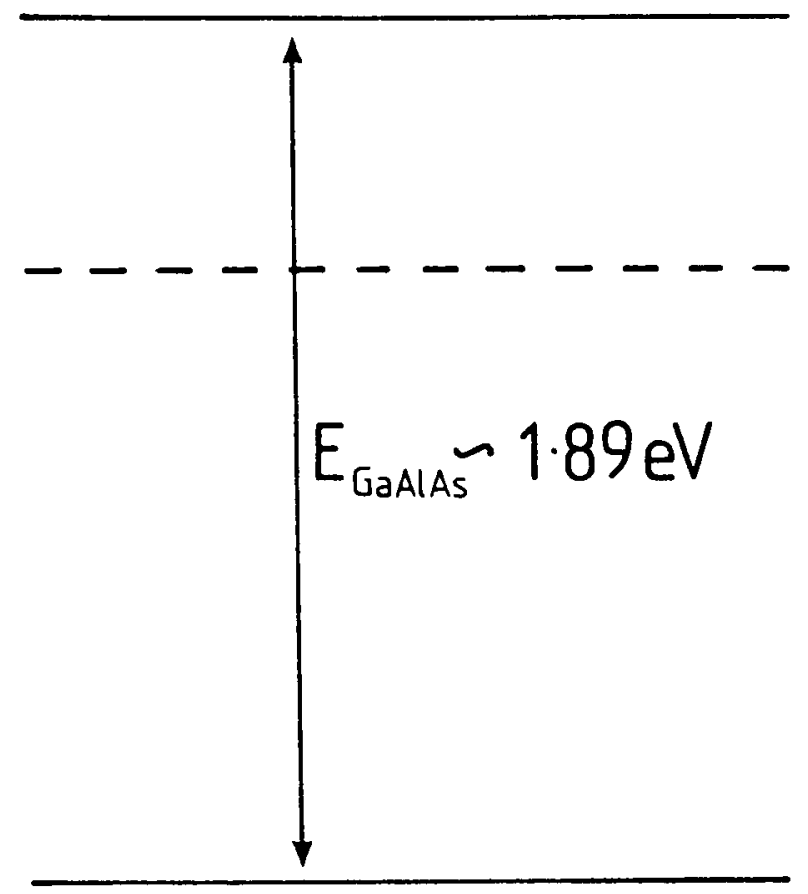

$\mathrm{Ga}_{07} \mathrm{Al}_{0.3} \mathrm{As}$

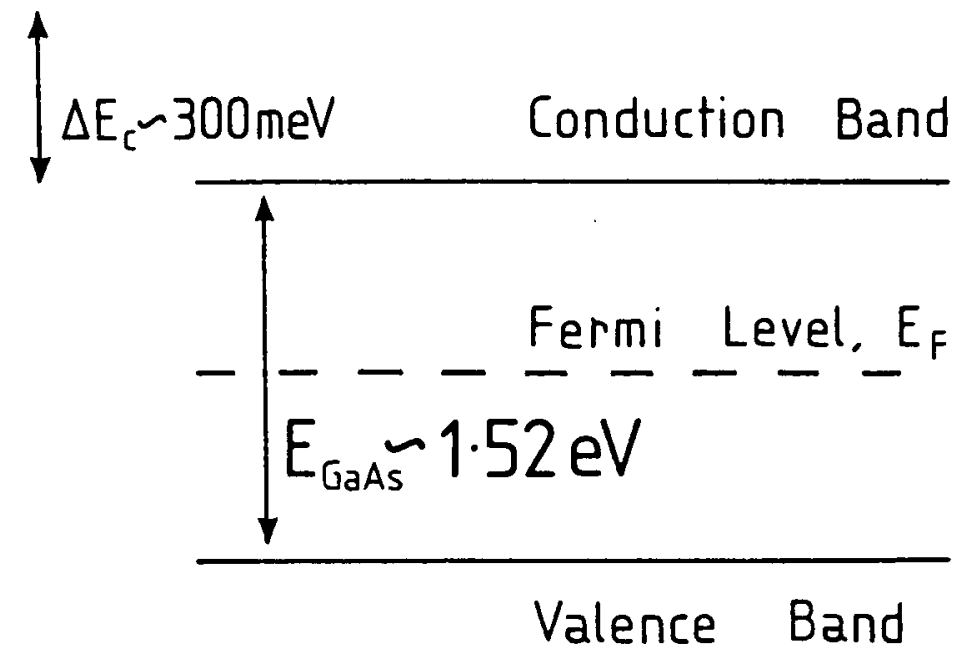

GaAs

(b)

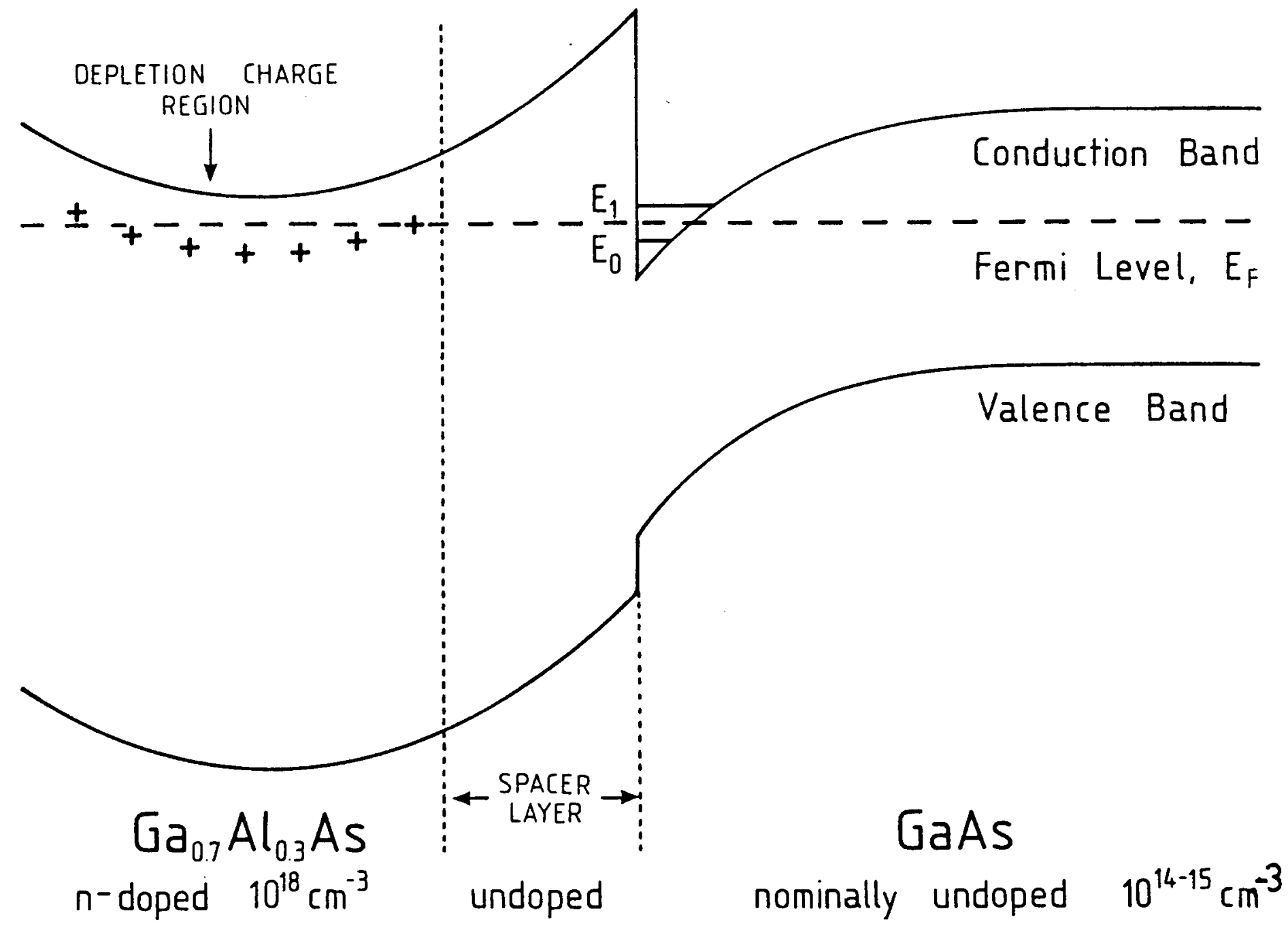

Figure 1.1 Formation of a heterojunction. A modulation doped structure is shown in which the undoped spacer layer separates the ionised donors from the $2 \mathrm{D}$ electron gas. 


\subsubsection{Modulation Doping}

The electrical quality of a structure is well described by the electron mobility, $\mu$. For electrons in the 2DEG the major limitation on $\mu$ at low temperatures comes from ionised impurity scattering and so the mobility can be greatly enhanced over the bulk value by modulation doping, whereby the electrons are spatially separated from their parent donors (Dingle et al. 1978). [At higher temperatures phonon scattering dominates the mobility, which is why many transport experiments are performed at liquid helium temperatures.] This is achieved by growing the narrow gap material as pure as possible and confining the $n$-type doping to the wide gap material. The mobility is further enhanced by growing a thin, undoped spacer layer of the wide gap material beween the interface and the doped region. Using this technique mobilities greater than $100 \mathrm{~V}^{-1} \mathrm{~s}^{-1} \mathrm{~m}^{2}$ can now be obtained routinely (Harris et al. 1986), with values an order of magnitude larger possible by chosing more subtle doping profiles (Foxon \& Harris 1989).

A thin GaAs capping layer is often grown on top of the GaAlAs layer to fix the surface potential and aid contacting, but it must be sufficiently thin to prevent a second 2DEG forming. Carriers can also be introduced into quantum wells by modulation doping on one, or both, sides of the well (Harris et al. 1988). Full details of the structure and properties of each sample will be given in the next chapter.

\section{$\underline{1.2 .3 \quad \text { Quantum Wells }}$}

Two heterojunctions formed back-to-back, ie. a thin layer of narrow gap material sandwiched between layers of wide gap material, constitutes a quantum well - Fig. 1.2a illustrates a narrow modulation doped well. If the intervening layer of narrow gap material is sufficiently thin, $\sim 100 \mathrm{~A}$, there will be very little band bending and the carriers will be bound by the band discontinuities in approximately square potential wells. There will also be a square well in the valence band in which a hole gas could be confined. In undoped wells, used in optical experiments where a population of charge carriers is not required, there will not be the same band bending as illustrated in

Fig. 1.2. Also it is possible to just dope the wells from one side, in which case they 
will appear asymmetric. As the well width is increased the band bending becomes more important and the structure will resemble a pair of heterojunctions - Fig. 1.2b. Often the well will be asymmetric and the ground state on one side will have a lower energy than the other, resulting in different carrier densities at the two interfaces. There may also be an excited state which will extend right across the well.
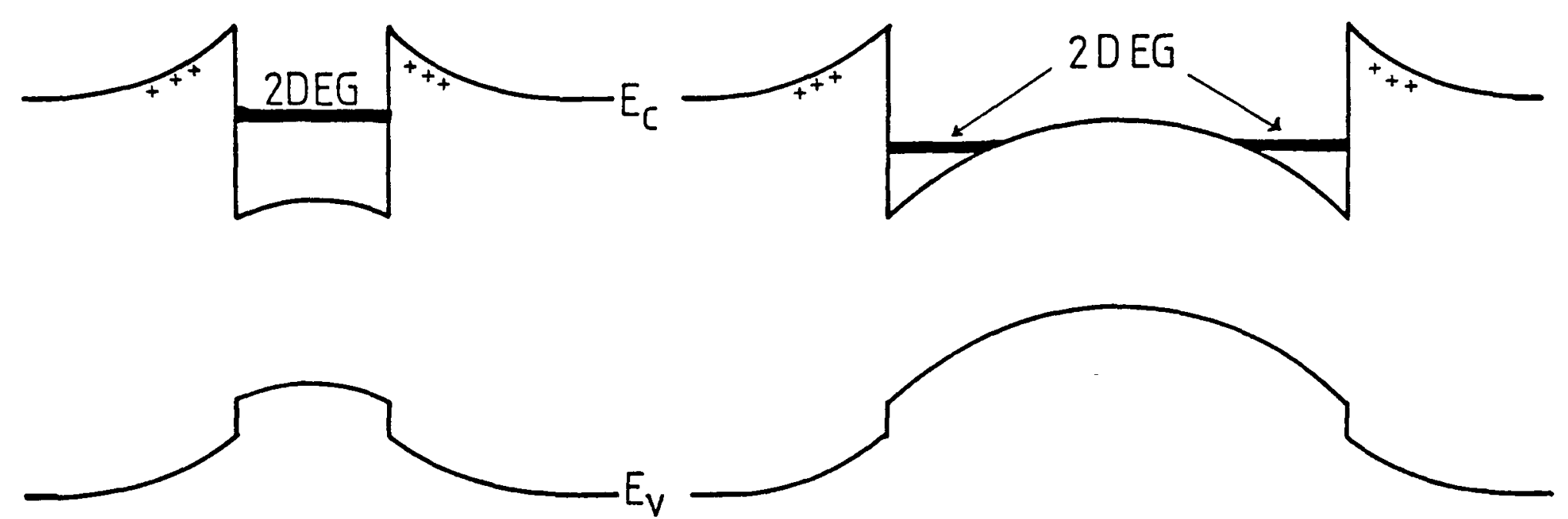

$\begin{array}{ll}\text { (a) Narrow Well } & \text { (b) Wide Well }\end{array}$

Figure 1.2 Band shape of a quantum well.

Often a series of quantum wells are grown, giving several layers of electrons, in a multi-quantum well structure (MQW). If the barriers between the wells are thin, the electronic wavefunctions from each well may overlap, and the structure is known as a superlattice, with the well spacing introducing a new periodicity into the system. We shall be mainly concerned with single heterojunctions and single quantum wells.

\subsubsection{Growth Techniques}

There are two widely used methods of growing semiconductor heterostructures, both of which can produce uniform layers of atomic thickness over regions of several $\mathrm{mm}^{2}$.

\section{(i) Molecular Beam Epitaxy}

MBE is a refined form of vacuum evaporation in which thermally generated atomic or molecular beams impinge on a heated substrate under ultra-high vacuum (UHV) conditions (Cho \& Arthur 1975, Joyce 1985). It is energetically favourable for atoms 
reaching the surface to adhere to the sides of imperfections and so, under suitable conditions, the crystal grows one atomic layer at a time. When growing GaAs by this method, the As atoms will only adhere to sites adjoining Ga atoms, but $\mathrm{Ga}$ atoms will adhere to any site. Thus, provided that an excess flux of As is maintained, it is possible to grow crystals of extremely high quality. Alloy compounds (such as AlGaAs) are grown by introducing a controlled flux of a third element and dopants are introduced in a similar way. In order to obtain sharp interfaces the sources are individually switched by electro- mechanical shutters, usually controlled by a computer. For GaAs, growth rates are typically one monolayer $(\sim 3 \AA)$ per second with a substrate temperature of $\sim 650^{\circ} \mathrm{C}$. Unfortunately, $\mathrm{MBE}$ is not very suitable for the growth of materials containing phosphorus as there are severe problems due to the phosphorus deposition on the inside of the apparatus and hence cross contamination.

\section{(ii) Metal-Organic Vapour Phase Epitaxy}

An alternative method is MOVPE - a form of metal-organic chemical vapour deposition (MOCVD) which results in epitaxial growth (Razeghi et al. 1982 \& 1983, Bass \& Young 1984). In this technique a carrier gas (usually hydrogen) transports alkyl compounds of the group III elements to a heated substrate where they react with hydrides of the group $\mathrm{V}$ elements. For example, GaAs would be grown using trimethylgallium, $\mathrm{Ga}\left(\mathrm{CH}_{3}\right)_{3}$, or triethylgallium, $\mathrm{Ga}\left(\mathrm{C}_{2} \mathrm{H}_{5}\right)_{3}$ and arsine $\left(\mathrm{AsH} \mathrm{H}_{3}\right.$. By adding a controlled flux of trimethylaluminium, the alloy AlGaAs can be grown. MOVPE can produce interfaces of similar, though slightly inferior, quality to MBE. However it is a more flexible technique since the growth of compounds containing phosphorus presents no major difficulties. There has been much debate amongst crystyal growers over the pressure that should be used. Some of the samples of this thesis were grown at atmospheric pressure (AP-MOCVD) and some at $76 \mathrm{mmHg}$ (LP-MOCVD), but were quite different structures and so no direct comparison could be made. Ougezzaden et al. (1989) have compared material grown from identical recipies in the same reactor vessel, using low- and high pressure techniques and find no obvious differences in either the surface quality or luminescene from the two materials. 


\subsubsection{Control of Electron Density}

There are a number of ways in which the electron density, $n_{s}$, can be changed in a modulation doped semiconductor heterojunction, each of which may affect $\mu$ by the way the scattering is changed:

(i) Changing the Spacer Layer Thickness - more electrons will be able to enter the 2DEG if there is a narrower spacer layer. This will also increase the remote ionised impurity scattering, as the scattering centres are brought closer to the electrons.

(ii) Increasing the Number of Donors - this could either be achieved by increasing the doping density or the width of doped material, and has a similar effect on the 2DEG as changing the spacer thickness.

(iii) Application of a Gate Voltage - a voltage between the surface of the material and the 2DEG will change the electrostatics of the potential well. A positive surface voltage increases $n_{s}$. This mechanism leaves the numbers of scatterers unaltered, but tends to squash the electrons towards the interface, which brings them closer to the impurities and would also accentuate any interface scattering. 'Back gates', with the voltage applied between the substrate and 2DEG, have also been used, especially for optical work where the front surface must not be disturbed, but have a smaller effect on $n_{s}$ than front gates.

(iv) Persistent Photoconductivity - electrons can be excited into the 2DEG by illumination with the red (or infra-red) light from an LED. Below $\sim 100 \mathrm{~K}$ the effect is persistent (Nelson 1977, Lacklison et al. 1988): at higher temperatures continuous illumination is required. There are a number of mechanisms responsible for this effect:

(a) Above band-gap radiation can excite electrons from the valance band of the narrow gap material (eg. GaAs in a GaAs-GaAlAs heterojunction), which then drop into the 2DEG. The holes created in this process recombine with acceptor states in the GaAs within $\sim 1 \mu \mathrm{m}$ of the interface. These states are metastable since re-ionisation is energetically favourable after overcoming an. 
initial ionisation barrier.

(b) Electrons may also be excited from deep trap states. In GaAs the DX centres play an important role (Lang et al. 1979). Changes to the scattering on photoexcitation will depend on the charge state of the traps, which may be initially neutral or negative.

Photodeexcitation has also been observed (Usher 1988), but the mechanism although thought to involve multiple stages is not well understood.

(v) Hydrostatic Pressure - under pressure the band structure changes, resulting in changes in $n_{s}$. In GaAs and GaInAs systems the carrier concentration decreases as the pressure is increased (Greorgis et al. 1988, Gauthier et al. 1988).

In this thesis samples of different spacer thickness will be used and the carrier concentration changed in a given sample by photoexcitation with a red LED.

\subsection{QUANTISATION IN HETEROSTRUCTURES}

This section will present some of the more important results for the energy levels and wavefunctions of electrons in semiconductor heterostructures. The treatment will be brief as this is now a standard problem shortly to appear in undergraduate textbooks.

\subsubsection{Electrical Quantisation - Subbands}

If the one-dimensional potential well confining the carriers is sufficiently narrow the motion perpendicular to the interface will be quantised whilst motion parallel to the interface will remain essentially free. The quantised levels for motion perpendicular to the interface are known as subbands, since each level corresponds to the lower edge of a two-dimensional dispersion relation for motion parallel to the interface. Since the thicknesses of the two-dimensional layers considered are $\sim 100 \AA$, which is large compared to the atomic scale, the effective mass approximation is justified.

Define the plane containing the interface as the $x y$-plane, the confining potential will ony depend on $z$, and so the spinless one electron Schrodinger equation is:

$$
\left[\frac{p^{2}}{2 m^{*}}+e V(z)+E_{k}\right] \psi_{k}^{\prime}=0
$$


where $\mathrm{p}$ is the electron momentum operator and $\mathrm{m}^{*}$ is the effective mass (assumed isotropic). This has solutions

$$
\begin{aligned}
\psi_{\mathrm{k}}(\mathrm{x}, \mathrm{y}, \mathrm{z}) & =\varphi_{\mathrm{i}}(\mathrm{z}) \exp \left\{\mathrm{i}\left(\mathrm{k}_{\mathrm{x}} \mathrm{x}+\mathrm{k}_{\mathrm{y}} \mathrm{y}\right)\right\} \\
\mathrm{E}_{\mathrm{k}} & =\mathrm{E}_{\mathrm{i}}+\frac{\hbar^{2}}{2 \mathrm{~m}^{*}}\left(\mathrm{k}_{\mathrm{x}}{ }^{2}+\mathrm{k}_{\mathrm{y}}{ }^{2}\right)
\end{aligned}
$$

and

where the wavefunction and energy of the $i^{\text {th }}$ subband, $\varphi_{i}(z)$ and $E_{i}$, satisfy the one-dimensional equation:

$$
\left[\frac{\hbar^{2}}{2 m^{*}} \frac{\partial^{2}}{\partial z^{2}}+e V(z)+E_{i}\right] \varphi_{i}(z)=0
$$

Thus, the electrons move freely in the $x y$-plane, and $k_{x} \& k_{y}$ remain good quantum numbers and, to a first approximation, the in-plane dispersion curve is parabolic. However, their motion in the $\mathrm{z}$ direction is now determined by Eqn. 1.4, with the potential $V(z)$ given by the solution of Poisson's equation

$$
\frac{\partial^{2}}{\partial z^{2}} V(z)=-\frac{\rho}{\epsilon_{\mathrm{r}} \epsilon_{0}}
$$

In this equation $\rho$ is the charge density due to: (i) space charge in the depletion regions of the higher gap material, due to the ionised donors; (ii) depletion charge in the lower gap material arising from residual impurities and (iii) the 2DEG itself, whose charge density is given by

$$
\rho_{2 D E G}=e \sum_{i} n_{i} \int \varphi_{1}^{*}(z) \varphi_{i}(z) d z
$$

where $n_{i}$ is the number of electrons in the $i^{\text {th }}$ electric subband. The eigenstates for z-motion must be found by solving Eqns. 1.4 - 1.6 self-consistently, which requires numerical calculations for complete solutions. The results of some of these including many-body exchange and correlation effects (Ando 1982a, Stern \& Das Sarma 1984) will be considered in Chapter 6 . For true two-dimensional behaviour all the electrons must be in the lowest subband, which requires the elecrtric quantum limit and the subband separation to be much less than the thermal energy. This latter condition will rarely be satisfied in the high temperature experiments of this thesis and so motion in the third 
direction, characterised by $\varphi_{\mathrm{i}}(z)$ and $E_{\mathrm{j}}$, may be important.

The density of states in a two-dimensional system has a particularly simple form:

$$
D(E)=\frac{m^{*}}{\pi \hbar^{2}} \quad \sum_{i} \delta\left(E>E_{i}\right)
$$

where a factor of 2 has been included to account for spin. For GaAs, with $\mathrm{m}^{*}=0.07 \mathrm{~m}_{\mathrm{e}}$ this has a value $2.92 \times 10^{10} \mathrm{~cm}^{-2} \mathrm{meV}^{-1}$

\section{$\underline{\text { Heterojunctions }}$}

One useful approximation in calculating the subband energy in heterojunctions is the variational calculation of Stern (1972), where only occupation of the lowest subband is considered (the electric quantum limit). He used the trial wavefunction, first proposed by Fang \& Howard (1966):

$$
\varphi_{0}(z)= \begin{cases}\left(b^{3 / 2}\right)^{1 / 2} z \exp (-b z / 2), & z \geq 0 \\ 0, & z<0\end{cases}
$$

where $b$ is a variational parameter. This gives the energy of the lowest electric subband as

$$
\mathrm{E}_{0} \approx \frac{5}{32}\left[\frac{33 \mathrm{n}_{0} \mathrm{e}^{2} \hbar}{\left(\mathrm{m}^{*}\right)^{\frac{1}{2} \epsilon \epsilon_{0}}}\right]^{2 / 3}
$$

and the mean distance of electrons from the interface as

$$
\mathrm{z}_{0} \approx \frac{3}{\mathrm{~b}} \approx 2\left[\frac{9 \epsilon \epsilon_{0} \hbar^{2}}{11 \mathrm{n}_{0} \mathrm{e}^{2} \mathrm{~m}^{*}}\right]^{1 / 3}
$$

where

$$
\mathrm{n}_{0}=\mathrm{n}_{\mathrm{s}}+\frac{32}{11} \mathrm{n}_{\text {dep }}
$$

$\mathrm{n}_{\mathrm{s}}$ is the $2 \mathrm{D}$ electron concentration and $\mathrm{n}_{\text {dep }}$ the depletion charge density.

The above calculation assumes that the electron wavefunction does not penetrate the barrier material. Whilst this is not strictly true for a heterojunction, such a simple analytic solution is useful, especially as it enables calculation of $\langle T\rangle_{z}$, the z-component of the kinetic energy. Penetration of the barrier has been considered in variational calculations by Ando (1982b) and Bastard (1983a \& 1984). Comparison of their results with those calculated above show that $E_{0}$ and $z_{0}$ are reduced by $10-15 \%$, although only about $1 \%$ of the electron density is in the barrier (Ando 1982b). 


\section{Quantum Wells}

For an isolated, undoped quantum well we can ignore the effects of space charge and simply use the finite square well model from elementary quantum mechanics to calculate the subband energies. The eigenvalues for motion in the plane perpendicular to the well are therefore given by

$$
\epsilon \tan \epsilon=\left[\frac{\Delta \mathrm{E}_{\mathrm{c}} \mathrm{m}^{*} \mathrm{a}^{2}}{2 \hbar^{2}}-\epsilon^{2}\right]^{1 / 2}
$$

for the even parity solutions, and

$$
-\epsilon \cot \epsilon=\left[\frac{\Delta \mathrm{E}_{\mathrm{c} \mathrm{m}^{*} \mathrm{a}^{2}}}{2 \hbar^{2}}-\epsilon^{2}\right]^{1 / 2}
$$

for the odd parity solutions, where $\epsilon^{2}=\mathrm{m}^{*} \mathrm{E}_{\mathrm{i}^{2}}{ }^{2} / 2, \Delta \mathrm{E}_{\mathrm{c}}$ is the conduction band discontinuity and $a$ is the width of the well (see, for example, Schiff 1968). It is clear that the subband energies are strong functions of the well width and thus a large measure of control over the subband structure is afforded by simply adjusting the layer thickness. As a result these structures have enormous device potential, particularly in opto-electronic applications where, for example, the bandgap can be tailored to give laser action at a particular frequency.

For a more realistic calculation it is necessary to take into account band bending, particularly in heavily doped structures with wide wells, and the difference in the effective masses of the two materials (Bastard 1983b).

\subsubsection{Quantisation in Magnetic Fields}

Most of the measurements in this thesis rely on the quantisation of electron energy levels in a magnetic field. Neglecting spin, the influence of a magnetic field may be accounted for by making the transformation $p \rightarrow p-e A$ in the Hamiltonian. A general magnetic field (in the yz-plane) can be represented in the Landau gauge $A=\left(-B_{y} z, B_{z} x, 0\right)$. The Hamiltonian is therefore given by

$$
H=\frac{1}{2 m^{*}}\left\{\left(p_{x}+e B_{y} z\right)^{2}+\left(p_{y}-e B_{z} x\right)^{2}+p_{z}^{2}\right\}+e V(z)
$$

Clearly $\mathrm{ip}_{\mathrm{y}}=\left[\mathrm{p}_{\mathrm{y}}, \mathrm{H}\right]=0$ so $\mathrm{p}_{\mathrm{y}}$ is a constant of the motion and can be replaced by 
$\hbar \mathbf{k}_{\mathbf{y}}$. Making the substitutions

$$
x^{\prime}=x-x_{0}=x-\frac{\hbar k_{y}}{m^{*} \omega_{c}}
$$

where $\omega_{c}$ is the cyclotron frequency for motion in the plane equal to $e B_{z} / m^{*}$; and $p_{x^{\prime}}=p_{x}$; the Hamiltonian can be rewriten in the form:

where,

$$
\mathrm{H}=\mathrm{H}_{1}+\mathrm{H}_{2}+\mathrm{H}_{3}+\mathrm{H}_{4}
$$

$$
\begin{aligned}
& \mathrm{H}_{1}=\frac{1}{2 \mathrm{~m}^{*}}\left(\mathrm{p}_{\mathrm{x}^{\prime}}{ }^{2}+\mathrm{m}^{* 2} \omega_{\mathrm{c}} \mathrm{x}^{2}\right) \\
& \mathrm{H}_{2}=\frac{e \mathrm{~B}_{\mathrm{y}}}{\mathrm{m}^{*}}(\mathrm{z}-<\mathrm{z}>) \mathrm{p}_{\mathrm{x}^{\prime}} \\
& \mathrm{H}_{3}=\frac{\mathrm{e}^{2} \mathrm{~B}_{\mathrm{y}}{ }^{2}}{2 \mathrm{~m}^{*}}\left(\mathrm{z}^{2}+<\mathrm{z}>^{2}-2 \mathrm{z}<\mathrm{z}>\right) \\
& \mathrm{H}_{4}=\frac{\mathrm{p}_{\mathrm{z}}{ }^{2}}{2 \mathrm{~m}^{*}}+\mathrm{eV}(\mathrm{z})
\end{aligned}
$$

The term $\mathrm{H}_{2}$, containing the factor $(z-<z>)$, should be small and if treated as a perturbation then the Hamiltonian is separable. We shall now consider different orientations of the magnetic field.

\section{(i) Perpendicular Fields}

In the usual experimental configuration the magnetic field is perpendicular to the plane of the 2DEG, ie. $\mathrm{B}_{\mathrm{y}}=0$, therefore $\mathrm{H}_{2}=\mathrm{H}_{3}=0$. This leaves $\mathrm{H}_{4}$, which gives the electric subband energies, and $\mathrm{H}_{1}$ which is the Hamiltonian for a simple harmonic oscillator of frequency $\omega_{\mathfrak{c}}$, giving rise to a set of discrete energy eigenvalues known as Landau levels. The energy levels are thus given by

$$
\mathrm{E}_{\mathrm{N}, \mathrm{i}}=\mathrm{E}_{\mathrm{i}}+\left(\mathrm{N}+\frac{1}{2}\right) \hbar \omega_{\mathrm{c}}
$$

where the positive integer $\mathrm{N}$ is the Landau index. The formation of discrete Landau levels has a profound effect on the density of states, transforming the even form of Eqn. 1.7 into a set of delta functions. Since $E_{N, i}$ is independent of $k$ these Landau 
levels will be highly degenerate. As the total number of states can not change and so each level must contain all the states that were in an energy range of $\hbar \omega_{c}$ in the absence of magnetic field. Thus from Eqn. 1.7 the density of states per unit area is:

$$
D(E)=2 \frac{\mathrm{eB}}{\mathrm{h}}
$$

in each Landau level and zero elsewhere. In 3-D the Landau levels are much broader because motion in the $z$-direction is unrestrained, giving an $E^{\frac{1}{2}}$ dependence to the density of states. However, in any real system the levels will be broadened by scattering and this will be discussed in Section 1.5 .

\section{(ii) Tilted Fields}

When a component of magnetic field is introduced parallel to the 2DEG [experimentally this is achieved by tilting the sample] the terms $\mathrm{H}_{2}$ and $\mathrm{H}_{3}$ are no longer zero. Treating $\mathrm{H}_{3}$ as a perturbation and gives energy levels:

$$
E_{N, i}=\left(n_{L}+\frac{1}{2}\right) \hbar \omega_{c}+E_{i}+\frac{e^{2} B_{y}^{2}}{2 m^{*}}\left(\left\langle z^{2}\right\rangle_{i}-\langle z\rangle_{i}^{2}\right)
$$

Thus the Landau level separation only depends on the component of the magnetic field along the z-direction, whilst the "parallel" field component changes the subband energies by an amount given by the last term in Eqn 1.23. This shift in the subband energies due to a parallel field was first observed by Tsui 1971 in tunneling experiments on InAs accumulation layers, and is responsible for subband depopulation in high fields which will be discussed in Section 6.5.

Although, the term $\mathrm{H}_{2}$ was neglected in arriving at Eqn. 1.23, it has important consequences since it couples motion in the $x y$-plane to that in the $z$-direction. Thus, although the motion of electrons in each subband is independent in perpendicular magnetic fields, application of even a very small parallel field component will cause anti-crossing type behaviour from the Landau levels of each subband. This subband mixing has been detected in cyclotron resonance measurements and may important for explaining some of the magnetophonon resonance data found in Chapters $3 \& 4$. 


\section{(iii) Spin Splitting}

So far only spinless electrons have been considered, however interaction of the magnetic field with the electronic spin causes each Landau level to be split into two spin-polarised levels, seperated in energy by $g^{*} \mu_{B} B$, where $g^{*}$ is the effective Landé g-factor. The resulting electron energy levels are thus given by:-

$$
\mathrm{E}_{\mathrm{N}}=\mathrm{E}_{\mathrm{i}}+\left(\mathrm{N}+\frac{1}{2}\right) \hbar \omega_{\mathrm{c}} \pm \frac{1}{2} \mathrm{~g}^{*} \mu_{\mathrm{B}} \mathrm{B}
$$

Each of these levels may then contain $e B / h$ electrons per unit area. It is useful to introduce the filling factor, $"$, which tells how many spin split levels are filled, eg $v=2$ means one completely full spinless Landau level. In the absence of electron-electron interactions $g^{*}=g$, the bare Landé $g$-factor, however when the many-body interactions are acounted for a much enhanced value of $g^{*}$ may result and this is measured in Section 6.4 .

Note that the spin splitting depends on the total magnetic field, whilst the Landau level separation $\left(\hbar \omega_{c}\right)$ only depends on the component of field perpendicular to the interface. This means that as the parallel component is increased the spin splitting will increase faster than the Landau splitting giving a point of 'coincidence', where they are equal, which may be used to determine $\mathrm{g}^{*}$.

\subsection{ADDITIONAL COUPLINGS AFFECTING THE BAND STRUCTURE}

The model used in the previous sections only considers non-interacting subbands and a rigid lattice. Interband couplings and lattice distortions may be accommodated within the effective mass approach by use of suitable perturbations:

\subsubsection{Band Non-parabolicity}

Deviations from a parabolic band occur near to band extrema due to interband couplings and can be calculated using k.p perturbation theory (Kane 1957). Using a three band k.p theory -1 conduction +2 valance bands - Palik et al. (1961) derived the relation:

$$
E(k)=E_{i}+\frac{\hbar^{2} k^{2}}{2 m^{*}}+\frac{K_{2}}{\bar{E}_{g}}\left[\frac{\hbar^{2} k^{2}}{2 m^{*}}\right]^{2}
$$


where $\mathrm{E}_{\mathrm{g}}$ is the band gap and the coefficient $\mathrm{K}_{2}$ depends on the band parameters. In an heterojunction this results in an increase in mass above the band edge value, $\mathrm{m}_{\mathrm{o}}^{*}$ :

$$
\frac{1}{\mathrm{~m}^{*}} \quad=\frac{1}{\mathrm{~m}_{\mathrm{o}}^{*}}\left\{1+\frac{2 \mathrm{~K}_{2}}{\mathrm{E}_{\mathrm{g}}}\left[\overline{\mathrm{E}}+\left\langle\mathrm{T}_{\mathrm{z}}\right\rangle\right]\right\}
$$

where $\bar{E}$ is the average energy above the conduction band edge of the electrons responsible for conduction. $\left\langle\mathrm{T}_{\mathrm{z}}\right\rangle$ represents the additional kinetic energy due to quantum confinement in the third direction and may be estimated from the variational wavefunction given in Eqn. 1.8 as

$$
\left\langle\mathrm{T}_{\mathrm{z}}\right\rangle=\frac{\hbar^{2} \mathrm{~b}^{2}}{8 \mathrm{~m}^{*}}
$$

Using the three band model $\mathrm{K}_{2}$ is predicted to be -0.9 in GaAs, however this model will only be accurate in materials where the band gap is much smaller than the separation between conduction bands (Hermann \& Weisbuch 1977). In GaAs where $E_{\mathrm{g}}=1.52 \mathrm{eV}$ and $\mathrm{E}_{1}=4.49 \mathrm{eV}$ a 5-band model is necessary (sometimes refered to as 14-band if the spin degeneracy is included). Braun \& Rössler (1985) and Zawadzki \& Pfeffer (1987) predict values of $\mathrm{K}_{2}$ of -1.1 and -1.5 respectively. Low temperature cyclotron resonance performed as a function of frequency $n$ similar GaAs-GaAlAs heterojunctions gave a good fit to $\mathrm{K}_{2}=-1.4$ (Hopkins et al. 1987).

\subsubsection{Polaron Coupling}

A polaron is the combination of an electron and its associated strain field in a polar crystal. The electron polarises its surroundings and the resulting lattice distortion is equivalent to a cloud of virtual longitudinal optic (LO) phonons. In a cyclotron resonance experiment, the polaron effect manifests itself by modifying the Landau level structure and hence the observed resonance positions. In magnetophonon resonance the coupling is between electrons and real LO phonons, but it is still the same polar coupling (Nicholas et al. 1989a). For a general discussion of polaron physics see Devreese (1972) and Devreese \& Peeters (1984). 


\section{Non-Resonant Polaron Coupling}

The Fröhlich Hamiltonian, which describes an electron interacting with a system of LO phonons, is given by

$$
H=\frac{p^{2}}{2 m^{*}}+\hbar \omega_{L O} \prod_{q}\left(a_{q}^{\dagger} a_{q}+\frac{1}{2}\right)+\left[\frac{4 \pi \alpha}{v}\right]^{1 / 2}\left[\frac{\hbar}{2 m^{*} \omega_{L O}}\right]^{4} \hbar \omega_{L O} \Gamma_{\dot{q}} \frac{1}{q}\left(a_{q} e^{i q \cdot r}-a_{q}^{\dagger} e^{-i q \cdot r}\right)
$$

(Fröhlich 1954), where $\omega_{\text {LO }}$ is the LO phonon frequency, $V$ is the volume of the crystal and $\mathrm{a}_{\mathrm{q}}^{\dagger}$ and $\mathrm{a}_{\mathrm{q}}$ are creation and annihilation operators respectively for an LO phonon of wavevector $q$. Magnetic field effects can be accounted for replacing $p$ by $(p-e A)$ in the first term. The Fröhlich coupling constant $\alpha$ is given by

$$
\alpha=\frac{\mathrm{e}^{2}}{4 \pi \epsilon_{0} \hbar}\left[\frac{\mathrm{m}^{*}}{2 \hbar \omega_{\mathrm{LO}}}\right]^{1 / 2}\left[\frac{1}{\epsilon(\infty)}-\frac{1}{\epsilon(0)}\right]
$$

where $\epsilon(0)$ and $\epsilon(\infty)$ are the static and high frequency dielectric constants respectively. For the semiconductors considered in this thesis $\alpha \ll 1$, eg. in GaAs $\alpha \approx 0.07$, and so, in the limit of small $\mathrm{q}$ and $\mathrm{B}=0$, Eqn. 1.28 can be solved perturbatively (Fröhlich 1954). This leads to a reduction in the energy by an amount $\Delta \mathrm{E}=\alpha \hbar \omega_{\mathrm{LO}}$ (the self-energy correction); and to an increase in the effective mass to $\mathrm{m}_{\mathrm{pol}}^{*}=\mathrm{m}^{*}(1-\alpha / 6)^{-1}$ (mass renormalisation).

For small magnetic fields and parabolic bands, Eqn. 1.28 can be solved by a variational method (Harper et al. 1973, Larsen 1964, 1972, Lindemann et al. 1983, Peeters et al. 1986), which yields the following expression for the energy difference between the ground state and the first excited Landau level

$$
\hbar \omega_{\mathrm{c}}=\left[1-\frac{\alpha}{6}\right]_{\mathrm{m}^{*}}^{\hbar \mathrm{eB}}-\frac{3 \alpha \hbar}{20 \omega_{\mathrm{LO}}}\left[\frac{\mathrm{eB}}{\mathrm{m}^{*}}\right]^{2}
$$

The first term is simply $\hbar \omega_{c}$ with $\mathrm{m}^{*}$ replaced by $\mathrm{m}^{*}{ }_{\mathrm{pol}}$ and second term represents the polaron contribution to the non-parabolicity. Rearranging this equation into the same form as Eqn. 1.26, gives the following approximate expression for the effective mass of a polaron in a weak magnetic field:- 


$$
\frac{1}{\mathrm{~m}^{*}}=\frac{1}{\mathrm{~m}_{\mathrm{pol}}^{*}}\left\{1+\frac{2 \mathrm{~K}_{2}^{\mathrm{pol}}}{\mathrm{E}_{\mathrm{g}}} \hbar \omega_{\mathrm{c}}\right\}
$$

where $\mathrm{K}_{2}^{\mathrm{ol}}$ is the quantity refered to in Section 1.4.1 and given by

$$
\mathrm{K}_{2}^{\mathrm{ol}}=-\frac{3 \alpha \mathrm{E}_{\mathrm{g}}}{40 \hbar \omega_{\mathrm{LO}}}
$$

The non-resonant polaron coupling is thus very similar to the effects of band non-parabolicity and so experimentaly these two are often indistinguishable and parameterised by a modified value of $\mathrm{K}_{2}^{\text {tot }}=\mathrm{K}_{2}^{\mathrm{np}}+\mathrm{K}_{2}{ }^{\mathrm{pol}}$.

In GaAs, the most comprehensive theoretical study by Peeters et al. (1986), including all transitions to higher levels, finds $\mathrm{K}_{2}{ }^{\mathrm{pol}}=-0.4$ in bulk and -1.0 in $2-\mathrm{D}$, however this value will be reduced by the finite extent of the wavefunction in the third direction and by screening. Hopkins et al. (1987) find a value of $\mathrm{K}_{2}^{\text {tot }}=-1.8$, corresponding to $\mathrm{K}_{2}{ }^{\mathrm{pol}}=-0.4$, fits their cyclotron resonance data on similar samples.

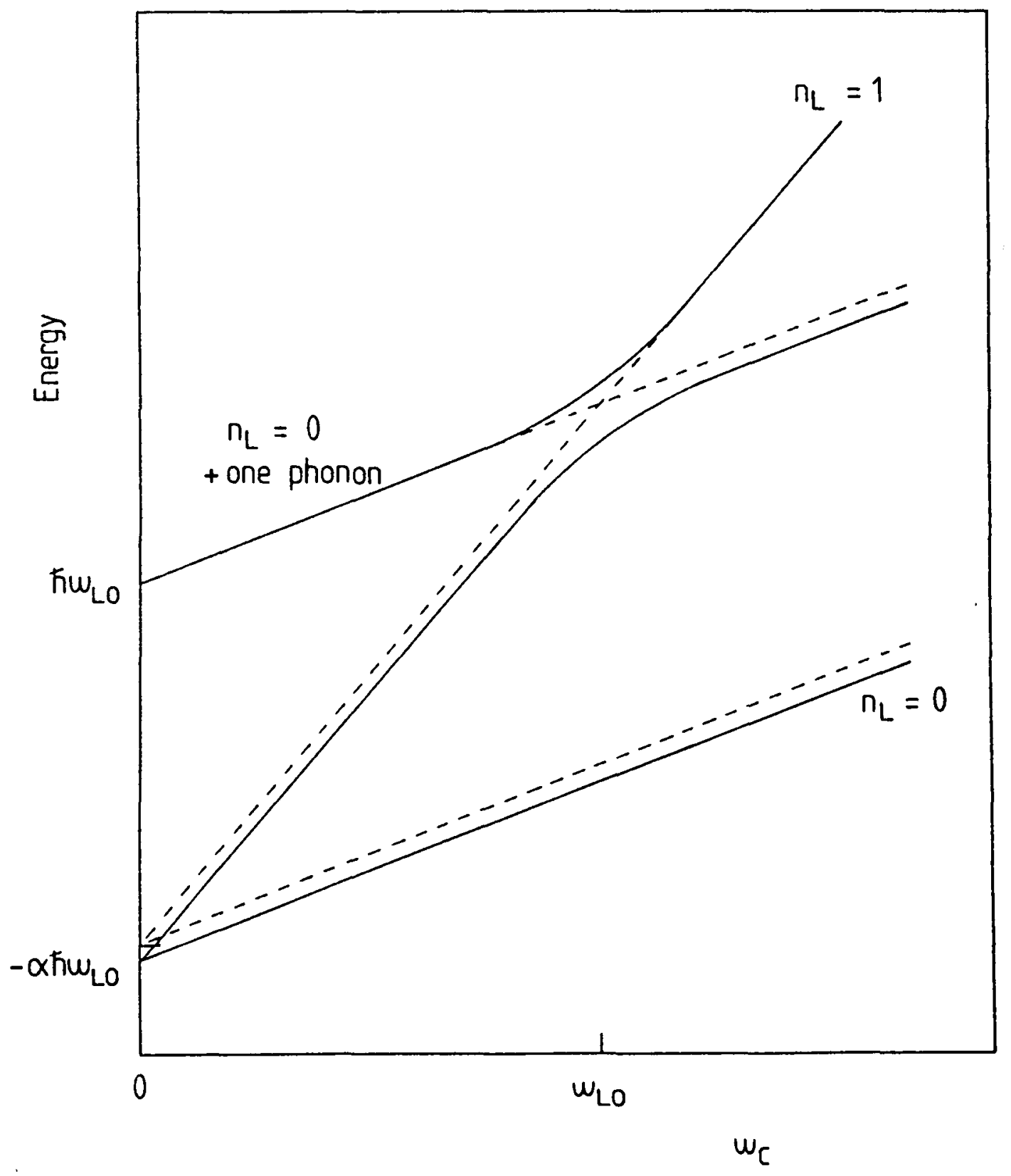

Figure 1.3 Energy of lowest two Landau levels as a function of magnetic field. Solid lines show the effect of polaron coupling (dashed lines are unperturbed levels). Resonant polaron coupling at $\omega_{\mathfrak{c}}=\omega_{L O}$ causes level anti-crossing. 


\section{Resonant Polaron Coupling}

At a sufficiently high magnetic field, the cyclotron energy is equal to the LO phonon energy and, in the absence of electron-LO phonon coupling, an electron in the $N^{\text {th }}$ Landau level would be degenerate with an electron in the $(N-1)^{\text {th }}$ level plus an optic phonon. The polaron interaction lifts this degeneracy to give hybridised states and anti-crossing behaviour, as illustrated in Fig. 1.3. By thus changing the band structure resonant polaron coupling alters the measured effective mass, the magnitude of which will be discussed in Section 3.4. Some recent temperature dependent cyclotron resonance results (Barnes et al. 1989). suggest resonant coupling may also be seen at harmonics of the phonon energy.

Two different theoretical approaches have been used in an attempt to describe the polaron energy spectrum in the resonant region. The first (Larsen 1966, 1972 \& 1974, Lindemann et al. 1983, Peeters \& Devreese 1985) relies on calculating the positions of the Landau levels, usually by treating the electron-LO phonon coupling term as a perturbation. Alternatively the cyclotron resonance spectrum may be calculated directly, using a memory function formalism (Peeters et al. 1986a, Wu et al. 1986 \& 1987).

\subsection{DENSITIES OF STATES IN 2-D}

\subsubsection{Broadening Mechanisms}

Up to now the Landau levels have been taken as $\delta$-function singularities. In practice they will be broadened by any processes which scatter the electrons. In the semiconductor heterojunctions studied in this thesis there are a number of possible mechanisms, which can be divided into two catergories: those which affect all electrons equally, homogeneous scattering; and those which are essentially local in character, inhomogeneous. There now follows a brief description of each [for theoretical descriptions see for example Rode (1975), or Walukiewicz et al. (1984)]:

(i) Phonon Scattering - At any finite temperature there will be some form of phonon scattering. There are three main mechanisms: deformation potential acoustic phonon coupling; piezzoelectric coupling to acoustic phonons; and the 
polar optic phonon interaction.

(ii) Ionised Impurity Scattering - Any ionised impurity in the system, deliberately introduced for doping or otherwise, will give rise to a Coulomb scattering potential. In modulation doped heterostructures these can be divided into two groups: remote impurities in the barrier material; and the background impurities, which are kept as low as possible by the growth procedure. In the Philips grown material there tend to be residual acceptor states in the GaAs.

(iii) Alloy scattering - In ternary compounds the alloy-disorder potential must be considered. In GaAs-GaAlAs the electrons are confined to the binary and so it is only the part of the wavefunction that penetrates the GaAlAs barrier $(\sim 5 \%)$ that will be subject to this potential. By contrast in GaInAs-InP the electrons are confined to the ternary and so will all be affected.

(iv) Interface Roughness - Any inhomogeneity in the hetero-interface or at the crystal surface will give rise to scattering. This process limits the mobility in $\mathrm{Si}$ MOS devices (Ando 1982b), but in MBE grown material the interfaces are usually extremely smooth. However, the so called 'inverted' interface of GaAs grown on GaAlAs turns out to much rougher than the 'normal' interface of GaAlAs on GaAs. Sakaki et al. (1987) suggest a roughness for the inverted interface of height $\sim 4 \mathrm{~A}$ and size $\sim 60 \mathrm{~A}$ and claims that this dominates the mobility in narrow quantum wells. This scattering is more important for MOVPE grown material.

The long range potentials amongst these - piezzoelectric, optic phonon, ionised impurity - can in addition be screened by the presence of a high electron density. Most interactions are also wavevector dependent, meaning that the electron Fermi wavevector, determined by the occupation number, must be taken into account.

\subsubsection{Localised or Extended?}

In a pure sample, all the electron states are equipotentials extended through the crystal. However, in the presence of a disorder potential, the equipotentials lying close to extrema in this potential will become closed loops and the electrons occupying these states will become localised and so unable to transport current through the sample. 
Since the mechanisms responsible for broadening the Landau levels and localising states are the same, it follows that the localised states are those in the tails of the levels. At $\mathrm{T}=0$ there may only be one extended state, but at higher temperatures, where $\mathrm{kT}$ exceeds the localising potential, more states will become delocalised. In general, transport measurements will only depend on the extended states, and so at low temperatures will reveal an approximately rectangular density of states in the centre of each Landau level (Usher 1988).

\subsubsection{The Shape of the Landau Levels}

Most theoretical treatments assume a form for the scattering and then calculate a shape for the density of states. Ando \& Uemura (1974) considered short range scattering and found an elliptical form for the density of states. This is not well behaved and produces unphysical structures in the conductivity if Landau levels are allowed to overlap. By considering higher order processes, eg multiple impurity scattering, a more reasonable Gaussian form results (Ando 1974a, Gerhardts 1975). This is now the conventional shape for inhomogeneous scattering. By contrast cyclotron resonance is a local transition and so will only be susceptible to homogeneous level broadening. Thus theories of cyclotron resonance lineshapes (eg Sarkar \& Nicholas 1982) predict a Lorentzian form for the density of states, with the linewidth given by the sum of widths due to each homogeneous scattering process.

Experimentally, the results are usually fitted to one of these forms, with the exact nature of the transitions involved determining the best shape.

\section{Density of States between the Landau Levels}

The existance of plateaux in the Quantum Hall Effect means that there must be states localised between the regions of extended states at the centres of the Landau levels. The dissappearance of the plateaux as the temperature is increased could give information about the distribution in energy of the localised states (Wei et al. 1985). This is more easily obtained by studying activated conduction. In these experiments the conductivity is measured as a function of temperature, which reveals an activation energy 
equal to the seperation of the Fermi energy from the extended states. The density of states profile is then deduced either by moving the position of the Landau levels with magnetic field, or by changing $E_{F}$ with carrier concentration. Although the conductivity is proportional to the density of extended states, it is the total density of states that is measured since this is what fixes $E_{F}$. Several experiments on samples of different qualities, as measured from the zero field mobility, (Weiss et al. 1986) show that there is a substantial density of states in the centre of the gap between Landau levels (ie at $v=2$ ). This was found to be larger in lower mobility structures - as much as $25 \%$ of the zero field density of states $\left(D_{0}\right)$ in a Si MOSFET (Gavrilov \& Kukushkin 1986) and $\sim 7 \% \mathrm{D}_{\mathrm{o}}$ in a high mobility GaAs heterojunction (Stahl et al. 1985). Predictions from a simple set of Gaussians would be more than 2 orders of magnitude too small, but using Gaussian peaks, with a width $\sim 1 \mathrm{meV}$, on a constant background fitted the data well. From studies of $\rho_{\mathrm{xx}}$ and the dissappearance of the plateaux in $\rho_{\mathrm{xy}}$ Wei et al. (1985) found a density of states in the gap of $\sim 1 \times 10^{10} \mathrm{~cm}^{-2} \mathrm{meV}^{-1}$ in a GaInAs-InP heterojunction, corresponding to a background of $\sim 50 \% \mathrm{D}_{0}$ !

The total density of states can be measured more directly by equilibrium measurements. Gornik et al. (1985) observed oscillations of the heat capacity in magnetic field, measuring changes in temperature of $\sim 30 \mathrm{mK}$ in response to $100 \mu \mathrm{s}$ heat pulses, and found the best fit to their data was from Gaussian peaks of $\sim 2 \mathrm{meV}$ width superimposed on a $20 \%$ background. Eisenstein et al. 1985 measured the magnetic susceptibility, using a torsion method giving deflections of $<10^{-4} \mathrm{rad}$, and modelled their data with a field dependent linewidth of $11 \mathrm{~K} / \mathrm{T}^{\frac{1}{2}}$. This data was also modelled by Gudmundsson \& Gerhardts (1987) with a $40 \%$ background density of states.

Smith et al. (1985 \& 1986) made magneto-capacitance measurements and found linewidths of $\sim 1 \mathrm{meV}$ and suggested the states in the mobility gap might be due to sample inhomegeneity. This idea was developed theoretically by Gerhardts \& Gudmundsson (1986) and Gudmundsson \& Gerhardts (1987), who considered local statistical fluctuations in the electron density and the chemical potential. These statistical models allowed a Gaussian distribution of $\delta \mathrm{n}_{\mathrm{s}}$ and $\delta \mu$ and produced finite 
densities of states between Landau levels, of a comperable value to the experimental results, with some kind of a physical justification rather than the arbitary flat background. Das Sarma \& Xie (1988) have performed a self consistent calculation for modulation doped heterostructures, where they allowed a random distribution of the remote ionised donors and include coupling between Landau levels. This also produces considerable densities of states between levels, and in fact, at low fields, shows the density of states in 2-D to be relatively featureless. By contrast, the photoluminescence measurements of Skolnick et al. (1988) gave an upper limit to the density of states between Landau levels of only $4 \% \mathrm{D}_{\mathrm{o}}$. This is because photoluminescence, like cyclotron and magnetophonon resonance, is a local transition and so will only be susceptible to sample inhomogeneities on the scale of the cyclotron radius. This maybe suggests that to model magnetophonon resonance experiments a rather small density of states is required in the Landau gap.

The importance of the exact form for the density of states is illustrated by calculations of the magnetophonon resonance position, which is shown in Section 3.2 to shift in opposite directions for different models. Although no concensus yet exists it seems clear that there is a substantial density of states betweeen Landau levels, which will be localised at low temperatures, but the actual amount detected depends on the method of measurement. A simple model to work with seems to be a set of Gaussian peaks superimposed on a flat background, with the fraction of states in the background an adjustable parameter. 


\title{
CHAPTER 2
}

\section{MAGNETO-TRANSPORT MEASUREMENTS}

\author{
AND
}

\section{SAMPLE CHARACTERISTICS}

$2.1 \quad$ Introduction

2.2 Magneto-transport

2.2.1 Transport in High Magnetic Fields 25

2.2.2 The Shubnikov-de Haas Effect 29

2.2.3 The Hall Effect 32

2.3 The Magnetophonon Effect

2.3.1 Overview 34

2.3.2 Calculations for Magnetophonon Resonance 36

$2.4 \quad$ Cyclotron Resonance

2.4.1 Theory $\quad 39$

2.4.2 Experiment 41

2.5 The Magnets 43

$\begin{array}{lll}2.6 & \text { Cryogenics } & 44\end{array}$

2.7 Electrical Measurements

2.7.1 Experimental Arrangement 45

2.7.2 Detection of Small Oscillations 47

2.8 Sample Parameters

2.8.1 GaAs-GaAlAs Heterojunctions 51

2.8.2 GaAs-GaAlAs Quantum Wells 54

2.8.3 GaInAs-InP Quantum Wells 55

2.8.4 GaInAs-InP Quantum Heterojunctions 57 


\section{$\underline{2.1 \quad \text { INTRODUCTION }}$}

This chapter will describe the basic experimental methods used for this thesis and give brief indications of how these can be modelled theoretically. The bulk of the measurements are of magnetoresistance: Shubnikov-de Haas oscillations at low temperatures; and magnetophonon resonance at higher temperatures. Cyclotron resonance was used to measure electron effective masses and the carrier densities were determined at elevated temperatures from the Hall coefficient.

The last section will describe the samples used in detail, so that in subsequent chapters they may just be refered to by number. I would again like to express my gratitude to all those involved in the preparation of these high quality samples, without which there could be no experimental results.

\subsection{MAGNETO-TRANSPORT MEASUREMENTS}

\subsubsection{Transport in High Magnetic Fields}

As was seen in Section 1.3.2 the application of high magnetic fields to a 2-D system completely quantises the electron motion into cyclotron orbits. For any electrical transport to occur these electrons must be able to scatter to orbits with different centre co-ordinates, $x_{0}$, which involves a change of the in plane wavevector, $k_{y}$. Thus high field conductivity will be proportional to the scattering rate, which will depend on the strength of the interaction and the density of empty states into which the electron can scatter ie. within $\sim \mathrm{kT}$ of the Fermi level for elastic scattering, although the final states may be further removed for inelastic photon/phonon assisted transitions.

Due to the singular nature of the density of states for a $2 D E G$ in a magnetic field the possible electron scatterings fall into two catergories: those involving transitions within a single Landau level; and inter-level scattering where initial and final states are in different Landau levels. The latter may be further complicated by scattering between states in different electric subbands. (In the remainder of this thesis the term subband will refer to electic subbands, and magnetic subbands will be called as Landau levels.)

In all the experiments described here the quantity measured is the sample resistivity. Unfortunately, most of the calculations are performed for conductivity, as this is usually 
more straight forward and historically was the property measured when experiments were performed on Corbino geometry samples. The resistivity must therefore be calculated from the conductivity from the tensor relation $\underline{\underline{\rho}}=\underline{\underline{\sigma}}^{-1}$. Thus:

$$
\rho_{x x}=\frac{\sigma_{x x}}{\sigma_{x x}^{2}+\sigma_{x y}^{2}}
$$

In high fields $\sigma_{x y} \gg \sigma_{x x}$ and so $\rho_{x x} \approx \sigma_{x x} J \sigma_{x y}{ }^{2}$. The off-diagonal conductivity increases linearly with $B$ and shows little structure, which means that oscillations in the resistivity will be proportional to those in the conductivity and thus the resistivity depends on the scattering rate. This is of course what happens in the abscence of magnetic field, but the indirect relationship makes for some counter intuitive situations in high fields. For instance both $\rho_{\mathrm{xx}}$ and $\sigma_{\mathrm{xx}}$ may be zero in the dissipationless states found when $\mathrm{E}_{\mathrm{F}}$ lies between filled Landau levels. This gives rise to some very interesting transport properties of the 2DEG as discussed below.

Magnetotransport theory has traditionally been based on application of the Boltzmann equation (Peierls 1931) to the distribution function $f(\mathbf{k})$ for electrons in the state $\mathbf{k}$

$$
\frac{1}{\hbar} \frac{\partial f(\mathbf{k})}{\partial \mathbf{k}} \cdot\left[\mathrm{e} \mathfrak{E}+\frac{\mathrm{e}}{\hbar} \frac{\partial \mathrm{E}_{\mathbf{k}}}{\partial \mathbf{k}} \wedge \mathrm{B}\right]+\frac{2 \pi}{\hbar} \sum_{\mathbf{k}^{\prime}} \mathrm{W}_{\mathbf{k} \mathbf{k}^{\prime}}\left[\mathrm{f}\left(\mathbf{k}^{\prime}\right)-\mathrm{f}(\mathbf{k})\right]=0
$$

where $\mathbb{E}$ is the electric field, $E_{\mathbf{k}}$ is the Bloch energy of state $\mathbf{k}$ and $W_{\mathbf{k k}}$ is the scattering probability from state $\mathbf{k}$ to state $\mathbf{k}^{\prime}$. The current is then obtained from

$$
\bar{j}_{\mu}=\frac{e}{\hbar} \int \frac{\partial E_{k}}{\partial \mathbf{k}_{\mu}} f(k) d^{3} \mathbf{k}
$$

Although originally developed for the limit of $\omega \tau<1$, the Boltzmann equation has been derived by Stinchcombe (1961) for arbitary $\omega \tau$ provided that (i) $\hbar \omega$ is much less than both the thermal energy and $E_{F}$ ie. magnetic quantisation is unimportant, and (ii) the scattering is both elastic and sufficiently weak so that $\hbar / \tau \ll E_{\mathrm{F}}$.

Outside these limits the Boltzmann equation is no longer valid and quantum effects become important (Stichcombe 1974) manifesting themselves in oscillatory behaviour of the transport coefficients in magnetic field. Adams \& Holstein (1959) and Argyres \& Roth (1959) developed transport theories by directly solving the equations for the density 
matrix, with the latter including inelastic scattering. This approach can be troublesome in high magnetic fields where there is no convinient representation in which to diagonalise the density matrix. Usually the Landau guage is used but unless the scattering is weak the off-diagonal components are not always small.

A alternative approach was made by Kubo based on his theory of irriversible processes (Kubo 1957), from which exact formal expressions for the conductivity arise. For static conductivity Kubo et al. (1965) give expressions like:

$$
\sigma_{\mathrm{xx}}=\frac{\mathrm{e}^{2}}{2 \mathrm{kT}} \int_{-\infty}^{\infty} \operatorname{Tr}<\rho_{0} \dot{\mathrm{X}}(0) \dot{\mathrm{X}}(\mathrm{t})>\mathrm{dt}
$$

where

$$
\mathrm{X}(\mathrm{t})=\exp [\mathrm{i} H \mathrm{t} / \hbar] \mathrm{X}(0) \exp [-\mathrm{i} H \mathrm{t} / \hbar]
$$

is the second quantised guiding centre coordinate operator. In order to extract any useful information from the Kubo formulae functional forms must be provided for $\rho_{0}$ and $H$ (the Hamiltonian, which may contain many perturbative terms) which entails certain approximations being made regarding the nature of the physical system. The scattering potential, $\mathrm{U}$ contained in $H$, automatically entres the Kubo formula to first order through the equation of motion:

$$
\dot{\mathrm{x}}=\frac{\mathrm{i}}{\hbar}[H, \mathrm{X}]=\frac{l^{2}}{\hbar} \cdot \frac{\partial \mathrm{U}}{\partial \mathrm{y}}
$$

and may also include dissipative scattering by phonons, which caused problems with the Boltzman approach.

One other possible treatment of dissipative transport in electron-phonon soupled systems involves the use of Feynman path integrals (Thornber \& Feynman 1970). This has been used by Peeters \& Devreese (1981) to find expressions for the electric field as a function of the average velocity of the electron system, $v$, via the momentum balance equation:

$$
\mathrm{e}[E+(\mathrm{v} \wedge \mathrm{B})]=\mathrm{F}(\mathrm{v})
$$

where $\mathbf{F}(\mathbf{v})$ is the force exerted on the electrons by interaction with the phonons and contains terms in the phonon occupation numbers as well as the electron density-density correlation function. The resistivity components are then obtained as follows:

$$
\rho_{x x}=\frac{v \cdot F(v)}{n(v e)^{2}} ; \quad \rho_{x y}=\frac{B}{n e}
$$


Figure 2.1 Typical experimental recording of the resistivity of a GaInAs-InP heterojunction. (a) $\rho_{\mathbf{x x}}$ - showing Shubnikov-de Haas oscillations with minima at the same magnetic fields as the Quantized Hall plateaux found in $\rho_{x y}-$ shown in (b).

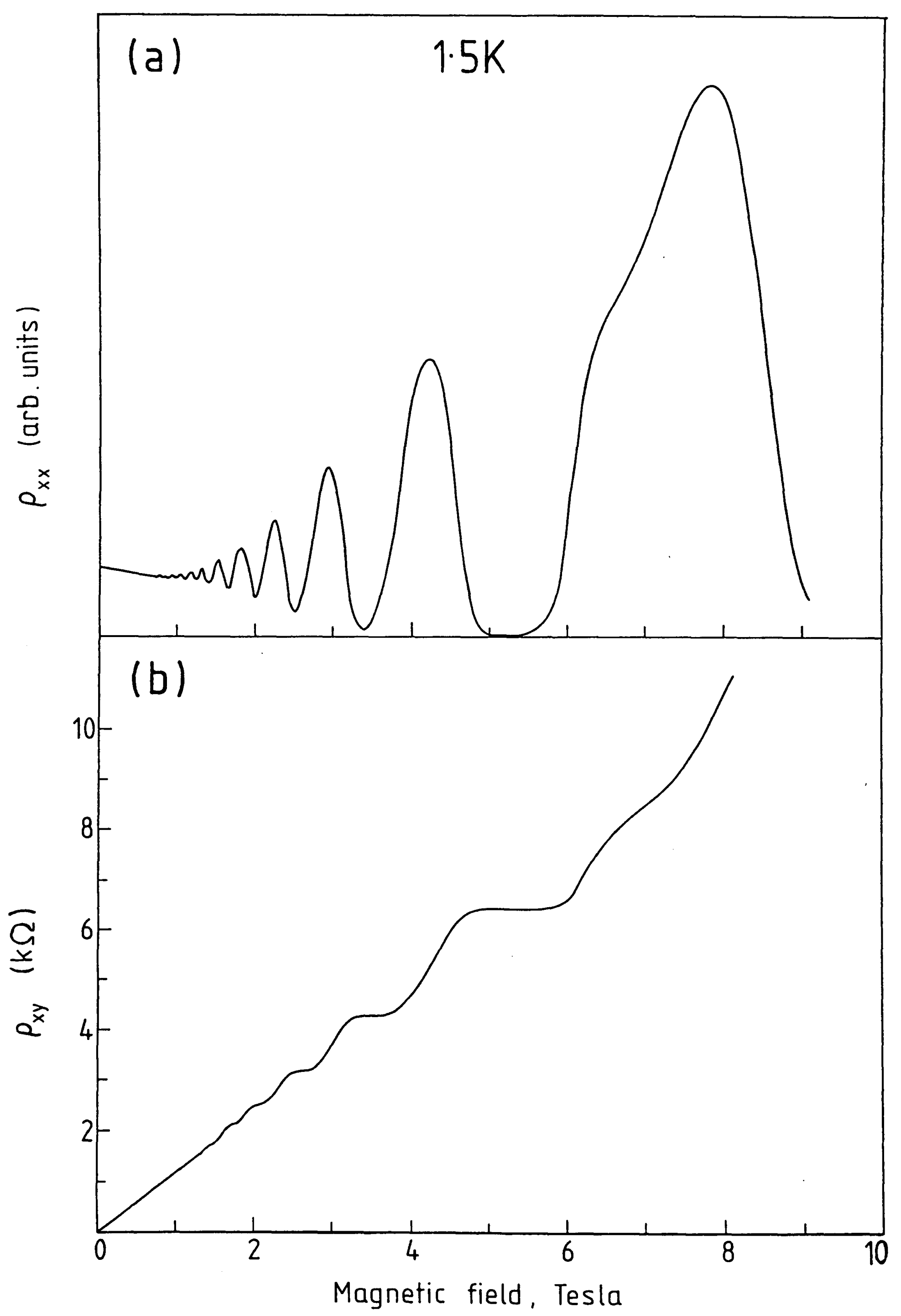




\subsubsection{The Shubnikov-de Haas Effect}

When a magnetic field is applied perpendicularly to a.2DEG Eqn. 1.21 shows that the energy of the Landau levels, and their separation, will increase linearly with the magnetic field. Now the Fermi energy, $E_{F}$, will be roughly constant with magnetic field, being fixed by the surface states, so as the magnetic field is increased the Landau levels will be swept through it. The density of states at the Fermi energy will thus vary, and the electrical conductivity will show oscillations periodic in inverse magnetic field. The resulting quantum oscillations are called Shubnikov-de Haas oscillations, after the researchers who first discovered the equivalent three-dimensional effect in Bismuth (Shubnikov \& de Haas 1930) and a typical set of oscillations are illustrated in Fig. 2.1a. The condition for observing these oscillations is that the Landau level separation must be much larger than both the thermal energy, $\left(\hbar \omega_{c} \gg k T\right)$ and the level broadening.

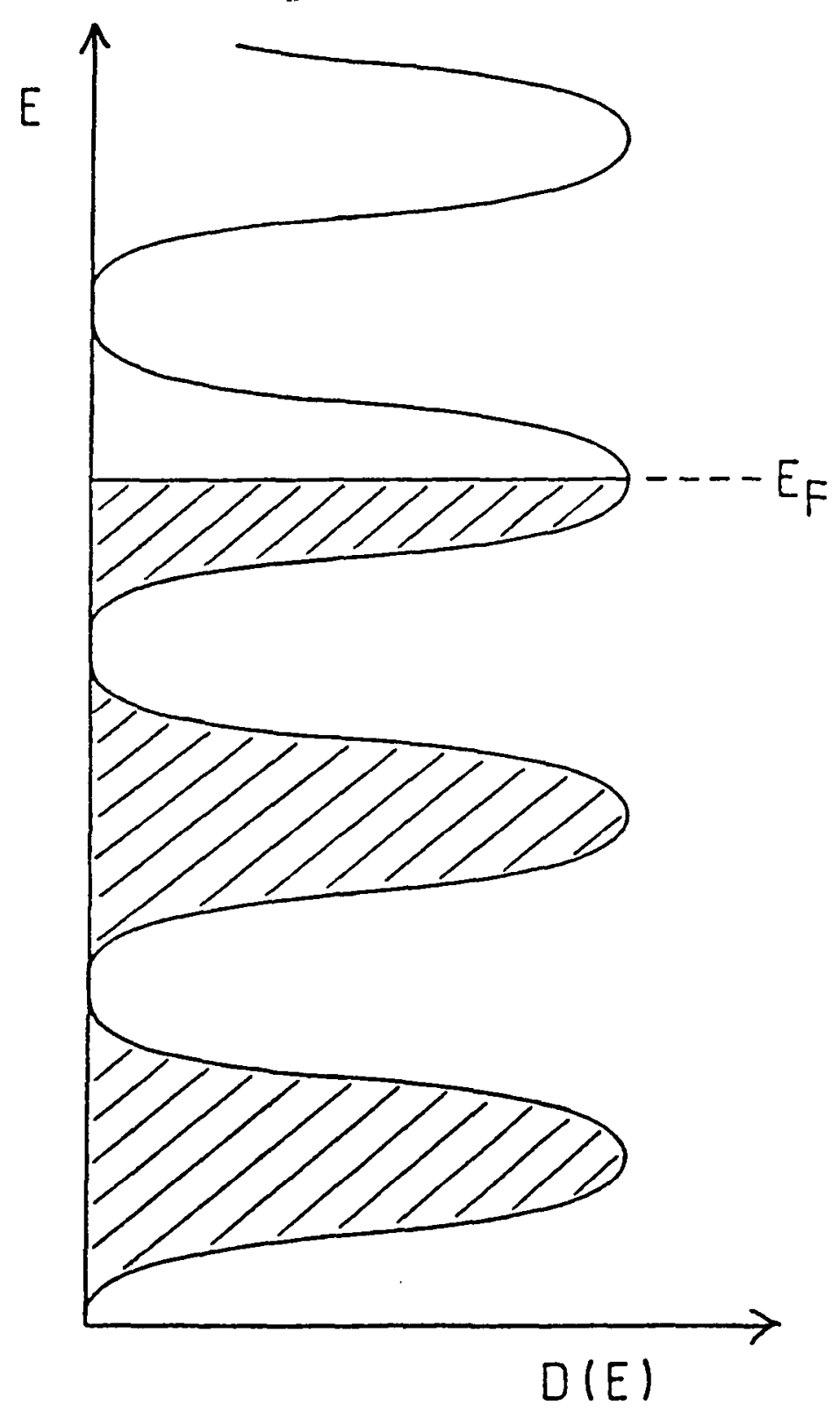

(a)

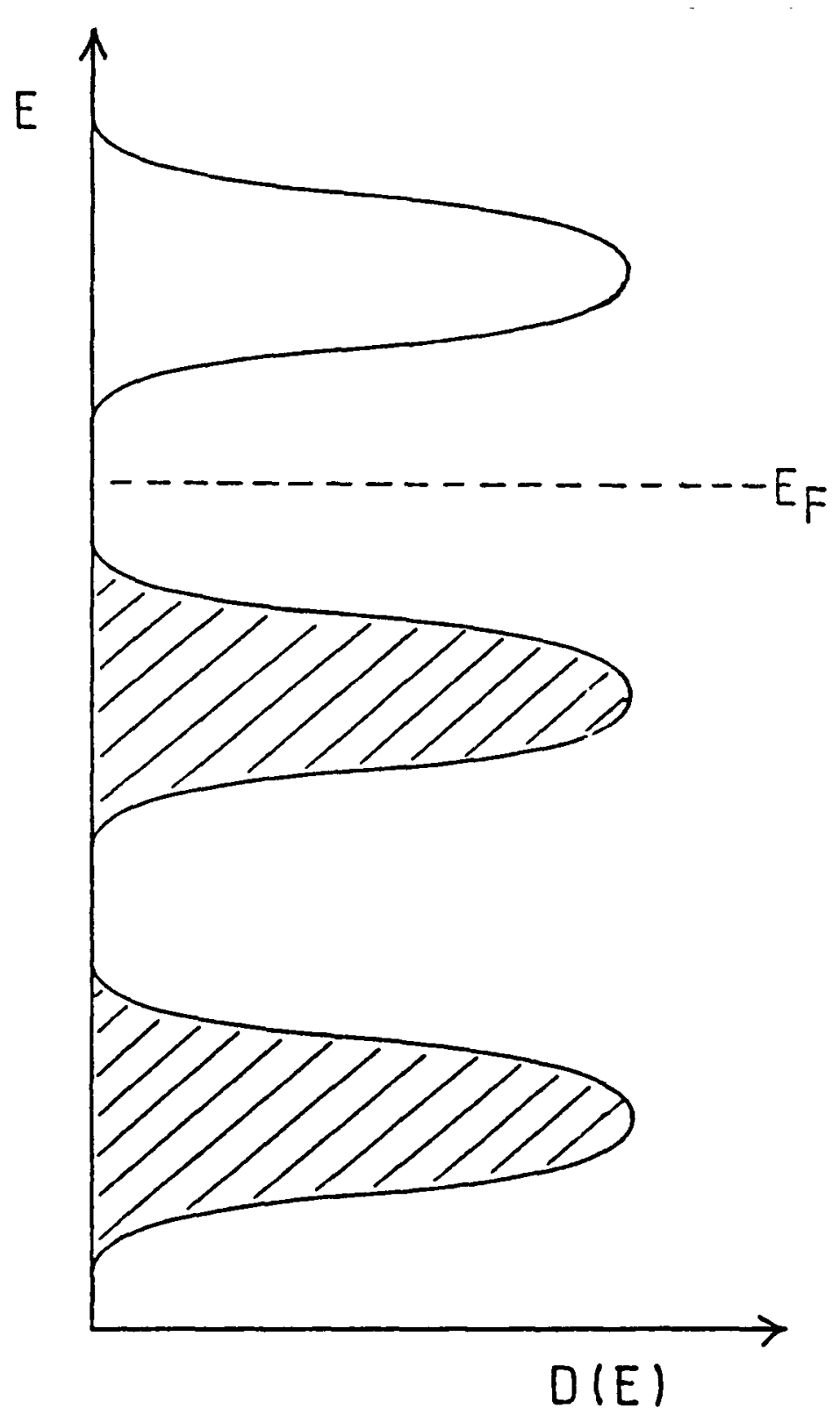

(b)

Figure 2.2 The density of states of a 2DEG in a magnetic field showing (a) metallic and (b) insulating situations, corresponding to maxima and minima in $\sigma_{x x}$. 
Figure 2.2 shows the (broadened) density of states for a $2 \mathrm{DEG}$ in two different magnetic fields, with the Fermi energy shown lying within a half filled Landau level (a) and between a completely filled level and a completely empty one (b). It can be seen from the numbers of empty states within $\sim \mathrm{kT}$ of $E_{F}$ that these represent the cases for maximum and minimum conductivity respectively, thus whenever an integral number of Landau levels are full the conductivity is a minimum. If there are no extended states between the Landau levels then the conductivity will actually be zero in this case. From Eqn. 1.22 with $\mathrm{N}$ Landau levels filled the carrier density in the system will be $\mathrm{n}_{\mathrm{s}}=\mathrm{N} 2 \mathrm{eB} / \mathrm{h}$, which gives a fundemental field for the Shubnikov-de Haas minima of

$$
B_{F}=\frac{n_{s} h}{2 e}
$$

$\mathrm{B}_{\mathrm{F}}$ represents a field above which all the electrons are in the lowest Landau level corresponding to the magnetic quantum limit. With $B_{F}$ in tesla the Shubnikov-de Haas effect may thus be used to calculate a sheet carrier density, in $\mathrm{cm}^{-2}$, as

$$
\mathrm{n}_{\mathrm{s}} \quad=0.484 \mathrm{~B}_{\mathrm{F}}
$$

\section{Ando's Formula}

Starting from the Kubo formula (Eqn. 2.4) and considering just short-ranged scattering in the self consistent Born approximation (SCBA) Ando (1974) found an expression for the conductivity demonstrating Shubnikov-deHaas oscillations. To first order is:-

$$
\sigma_{\mathrm{xx}}=\left[\frac{\sigma_{0}}{1+\left(\omega_{\mathrm{c}^{\top} \mathrm{s}}\right)^{2}}+\sigma_{\mathrm{osc}} \cos \left[\frac{2 \pi \mathrm{E}_{\mathrm{E}}}{\hbar \omega_{\mathrm{c}}}+\varphi\right]\right]
$$

where $\sigma_{0}$ is the conductivity at $\mathrm{B}=0$ and the amplitude of the oscillatory term is

$$
\sigma_{\text {osc }}=\frac{\sigma_{0}}{1+\left(\omega_{c^{\tau} s}\right)^{2}} \quad \frac{\chi}{\sinh \chi} \quad \mathrm{P}\left(\mathrm{B}, \tau_{\mathrm{s}}\right) \exp \quad\left(-\frac{\pi}{\omega_{\mathrm{c}} \tau_{\mathrm{s}}}\right)
$$

with $\lambda=\frac{2 \pi^{2} \mathrm{kT}}{\hbar \omega_{\mathrm{c}}}$

and

$$
P\left(B, \tau_{s}\right)=\frac{2 \pi\left(\omega_{c^{\top}}\right)^{2}}{1+\left(\omega_{c} \tau_{s}\right)^{2}}
$$

is a pre-exponential factor resulting from the self-consistency. This formula was considered valid in the low temperature limit where $\Delta \sigma \ll \sigma$. 
Subsequent workers have inverted the conductivity tensor (Eqn. 2.1) assuming:

$$
\sigma_{\mathrm{xy}}=-\omega_{\mathrm{c}^{\tau}} \sigma_{\mathrm{xx}}
$$

which is valid for $\mu \mathrm{B} \gg 1$, to give

$$
\frac{\Delta \rho_{x x}}{\rho_{x x}}=f\left(\omega_{c} \tau\right) \quad \frac{\chi}{\sinh \chi} \exp \left(-\frac{\pi}{\omega_{c} \tau_{s}}\right) \cos \left[\frac{2 \pi E_{F}}{\hbar \omega_{c}}\right]
$$

where $\rho$ is the background resistivity (constant in this model) and $f\left(\omega_{c} \tau\right)=P / \pi$. The value that should be used for $\tau$ is not clear. Certainly in the exponential the single particle lifetime $\left(\tau_{S}-\right.$ Chapter 7$)$ is required, but in the prefactor there are arguements for either this or the zero-field relaxation time, $\tau_{t}$, which appears in the original Ando paper. The descrepancy is particularly striking in modulation doped structures where the scattering is predominately small-angled from the remote impurities, making an order of magnitude difference between the two characteristic times. In contrast the theories only use a single, field independent, relaxation time. Coleridge et al. (1989) have considered the magnetic field dependent, Lorentzian broadened, density of states and using:

$$
\sigma_{\mathrm{xy}}=\mathrm{e} \frac{\partial \mathrm{N}}{\partial \mathrm{B}}-\omega_{\mathrm{c}} \tau \sigma_{\mathrm{xx}}
$$

where $\mathrm{N}$ is the number of states below $E_{F}$, find $f=2$ (avoiding the confusion of which $\tau$ to use) and that Eqn. 7.6 is valid for all $\omega_{\mathrm{c}^{\top}}$ until $\Delta \rho \sim \rho$ ie. before any flat bottomed minima appear in the magnetoresistace. However, their calculations, based on those of Isihara \& Smrčka (1986), assume a constant Fermi energy and allow the electron concentration to vary, which may well be true for surface accumulation layers, but in these modulation doped structures the picture is not so clear.

At sufficiently high magnetic fields, the spin-splitting of the Landau levels (Eqn. 1.24) will become comparable to the level separation and the oscillations will apparently double in frequency, as twice as many distinct levels pass through $E_{F}$.

Other complications arise when more than one subband lies below the Fermi energy, giving rise, at low fields, to independent series from each subband of periodicity $B_{F i}=n_{i} h / 2 e$, corresponding to the $n_{i}$ carriers in each subband. At higher magnetic fields the magnetoresistance becomes more complicated due to movement of $E_{F}$ between the subbands. Chapter 6 is devoted to the study of multiple subband occupation. 


\subsubsection{Hall Effect}

\section{(i) Measurement of Carrier Densities}

The Hall resistivity of a single channel, in which conductivity is by one type of carrier, is $\rho_{x y}=B / n_{s} e$. This is true at all temperatures and so can be used to measure the carrier concentration, even when Shubnikov-de Haas oscillations are not resolved. When more than one $2 D E G$ is populated the Hall voltage will depend on the total number of carriers present, whereas the periodicity of Shubnikov-de Haas oscillations is determined by the number in each 2DEG. Thus comparison between $\mathrm{n}_{\mathrm{Hall}}$ with $\mathrm{n}_{\mathrm{SdH}}$ could, for instance, determine the number of layers contacted to in a multi-quantum well.

However, measuring carrier densities from the Hall voltage has its problems: too small a voltage will be measured if the Hall voltage contacts are not at the edge of the sample and so lead to over estimates of the carrier density - in a given sample this will be a systematic error, which can be corrected for by comparison of $n_{\text {Hall }}$ and $n_{S d H}$ at $4.2 \mathrm{~K}$; at high temperatures there is often a contribution from parallel bulk conduction which will also tend to reduce the measured Hall voltage.

With two carrier types present of respective densities $n_{1}$ and $n_{2}$ and mobilities $\mu_{1}$ and $\mu_{2}$ the Hall coefficient has been calculated (Smith 1978) as:

$$
R=\frac{\rho_{x y}}{B}=-\frac{1}{e \cdot \frac{\left(n_{1} \mu_{1}^{2}+n_{2} \mu_{2}^{2}\right)+\mu_{1}^{2} \mu_{2}^{2}\left(n_{1}+n_{2}\right) B^{2}}{\left(n_{1} \mu_{1}-n_{2} \mu_{2}\right)^{2}+\mu_{1}^{2} \mu_{2}^{2}\left(n_{1}+n_{2}\right)^{2} B^{2}}}
$$

This shows the well known result as $B \rightarrow 0$ and in the high field limit gives $\mathrm{R}=-1 / \Sigma \mathrm{n}_{\mathrm{i}} \mathrm{e}$. The quadratic terms in Eqn. 2.14 give rise to deviations from a straight line when $\rho_{x y}$ is measured as a function of $B$, which is a useful indication of multi-channel transport. Notice that this equation has no linear terms in $\mathbf{B}$, so any asymmetry between recordings of $\rho_{x y}$ with the direction of magnetic field reversed are due to mixtures of the $\rho_{x x}$ - usually caused by the pairs of voltage contacts not being exactly opposite each other. To remove this source of error averages were taken of traces with $\pm \mathbf{B}$. 
The diagonal component of the conductivity is calculated, with $\sigma_{\mathrm{T}}=\Sigma \mathrm{n}_{\mathrm{i}} \mathrm{e} \mu_{\mathrm{i}}$, as:

$$
\sigma_{\mathrm{xx}}=\frac{\sigma_{\mathrm{T}}^{2}+\mu_{1}^{2} \mu_{2}^{2}\left(\mathrm{n}_{1}+\mathrm{n}_{2}\right)^{2} \mathrm{e}^{2 \mathrm{~B}}{ }^{2}}{\sigma_{\mathrm{T}}+\mu_{1} \mu_{2}\left(\mathrm{n}_{1} \mu_{2}+\mathrm{n}_{2} \mu_{1}\right) \mathrm{e}^{2 \mathrm{~B}}{ }^{2}}
$$

The different types of carriers could be electrons in different subbands - as in Chapter 6; or mixed electrons and holes. In the latter case, $\left(n_{1}, \mu_{1}\right)$ should be replaced by $\left(\mathrm{n}, \mu_{\mathrm{e}}\right)$ and $\left(\mathrm{n}_{2}, \mu_{2}\right)$ by $\left(-\mathrm{p}, \mu_{\mathrm{h}}\right)$, which gives $\rho_{x y} \propto 1 /(\mathrm{p}-\mathrm{n})$ in the high field limit.

\section{(ii) Quantised Hall Effect}

When an integral number of Landau levels, $p$ (counting spin-split levels seperately), are filled the carrier density is $\mathrm{peB} / \mathrm{h}$ and so at these points

$$
\rho_{\mathrm{xy}}=\frac{1}{\mathrm{p}} \frac{\mathrm{h}}{\mathrm{e}^{2}}
$$

It is also found that, in the presence of mild disorder, plateaux form in $\rho_{x x}$ which are accurately quantised at the values given by Eqn. 2.16 as shown in Fig. 2.1b. This is the famous Quantum Hall Effect (QHE) first reported by von Klitzing, Dorder \& Pepper in 1980, which earned von Klitzing the Nobel Prize for demonstrating the incredible accuracy of the quantisation - measured to better than 1 part in $10^{8}$ and now used as a standard of resistance.

The formation of plateaux, when $E_{F}$ lies between Landau levels, relies on the Landau levels being broadened with some states localised in the tails. The disorder potential is responsible for localising states, but also causes electrons in extended states to be accelerated when passing close to the localised states. They are therefore able to exactly compensate for the failure of the localised states to carry any current. Provided all the extended states in a Landau level are occupied the Hall resistivity will be exactly $\mathrm{h} / \mathrm{pe}^{2}$. As the occupancy increases $E_{F}$ will move through the localised states in the Landau level tail and $\rho_{x y}$ will not change, until $E_{F}$ gets to within $\sim k T$ of the extended states in the next Landau level. Thus the plateaux will narrow as the temperature is increased, or the disorder decreased. Obviously if there is too much disorder all the states will be localised and there will be no transport. 
In very pure samples at extremely low temperatures $(\sim 100 \mathrm{mK})$ additional plateaux form between the QHE plateaux at positions described by fractions $p / q$ rather than the integers p. This is the Fractional QHE (FQHE) which is due to correlated motion of the electrons, condensing out of the 2DEG to form a Fermi liquid. Study of the FQHE has evoked great passion amongst the rival workers, but I promise not to mention this effect again!

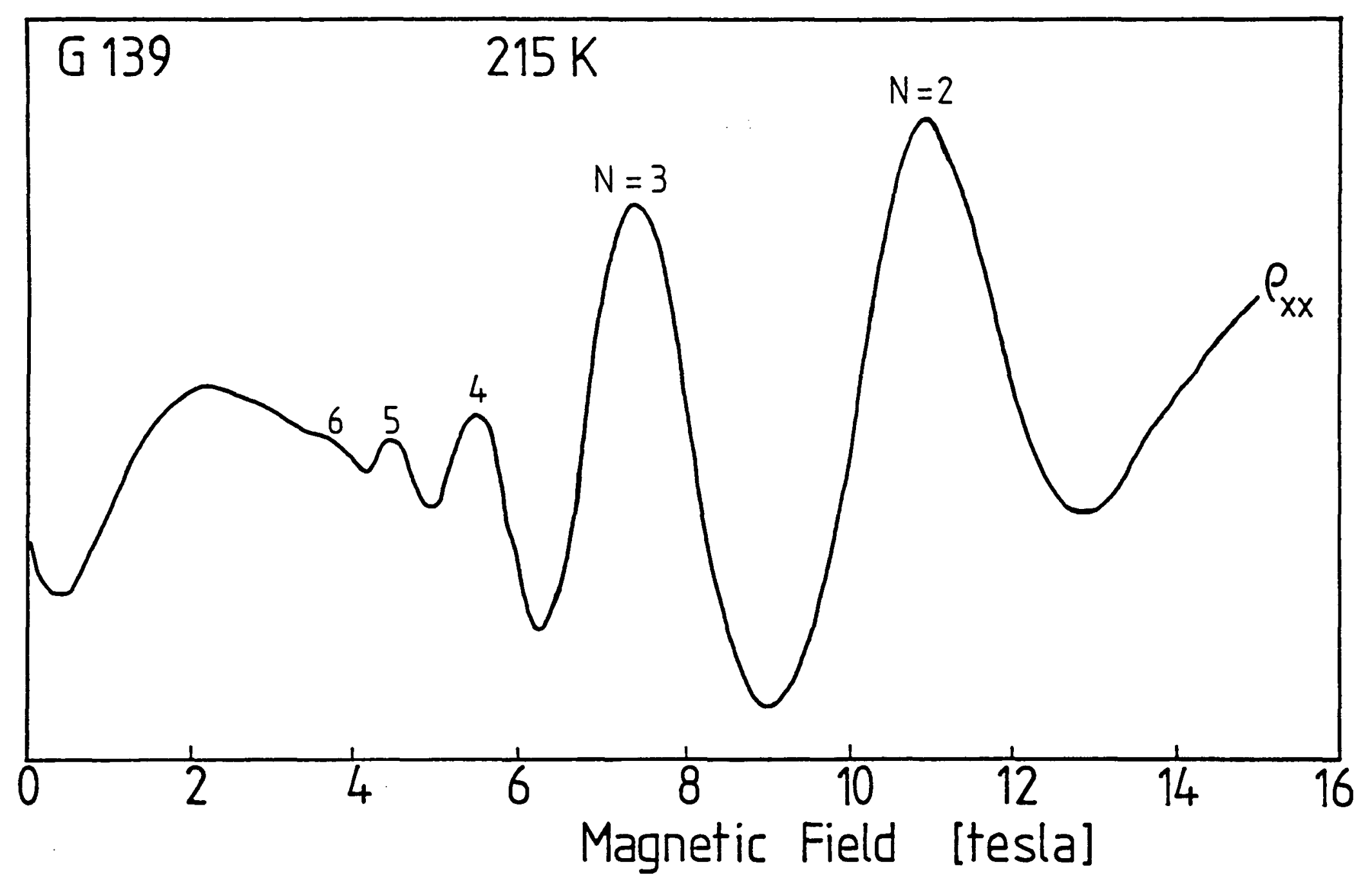

Figure 2.3 Experimental recording of magnetophonon resonance at $215 \mathrm{~K}$ in a low density GaAs-GaAlAs heterojunction.

\subsection{THE MAGNETOPHONON EFFECT}

\section{$\underline{2.3 .1 \quad \text { Overview }}$}

Electrons may resonantly absorb optic phonons whenever the phonon energy is equal to an integral number of Landau level separations. This leads to relaxation of the electron momentum and hence an increase in the resistivity at the resonance condition

$$
\hbar \omega_{0}=N \hbar \omega_{c}=N \hbar \frac{e B}{m^{*}}
$$


A series of maxima periodic in $1 / B$ with fundemental field $B_{F}=N B_{N}$ thus appear in the magnetoresistance - Fig. 2.3 is.typical - which can be used to deduce $\mathrm{m}^{*}$ or $\omega_{0}$ if the other is known. In the past most workers have assumed coupling is to the bulk longitudinal optic (LO) phonon and used magnetophonon resonance to measure the effective mass. In Chapters $3 \& 4$ the mass will be calculated from that measured in cyclotron resonance and some suprising information deduced regarding the coupling, which is not always found at the LO phonon energy.

It can be seen from Fig. 2.3 that the resonances form a damped series of oscillations which can be descibed empirically by the expression, first found by Stradling \& Wood (1968):

$$
\Delta \rho \quad \propto \exp \left(-\tilde{\gamma} B_{F} / B\right) \cdot \cos \left(2 \pi B_{F} / B\right)
$$

where $\tilde{\gamma}$ is an empirical damping factor, which will be shown below to be related to the broadening of the Landau levels. A study of this and the absolute values of the resonance amplitudes should yeild information about the important scattering mechanisms and the strength of the electron-phonon interaction. This forms the rest of Chapter 3.

The process of phonon emission can also occur, if an electron has sufficient energy, at the same resonance condition. The associated energy relaxation leads to resonant cooling of the electrons and a series of oscillations in the magnetoresistance, with minima at the resonance condition. This is known as "Hot Electron Magnetophonon Resonance" (HEMPR) and is studied in Chapter 5.

Magnetophonon resonance experiments are usually performed in the magnetic quantum limit: at higher fields than the Shubnikov-de Haas oscillations. Oscillations from these two processes are both periodic in 1/B and may appear similar, but can be distinguished firstly by their temperature dependences - Shubnikov-de Haas oscillations decrease monotonically with increasing temperature, while magnetophonon resonances have a peak amplitude around $150 \mathrm{~K}$; and also by their response to a change in carrier concentration - the Shubnikov-de Haas periodicity increases proportional to $n_{s}$, whereas that for magnetophonon resonance is expected to stay roughly constant, only changing through small changes in the mass which should be a second order effect. 


\subsubsection{Calculations for Magnetophonon Resonance}

There have been several calculations of the magnetoresistance which include magnetophonon resonances [see Peterson 1975 for review of 3-D work]. The first of these was by Gurevich \& Firsov in 1961 who used the Kubo formula and found logrithmic divergences in the conductivity at the resonance condition. Barker (1972) also used a perturbation expansion of the Kubo formula to calculate the amplitude of magnetophonon oscillations in bulk semiconductors. By assuming Lorentzian broadened Landau levels of width $\Gamma$, which could vary with magnetic field, he was able to remove the divergences and found the oscillatory part of the magnetoresistance to be:

$$
\Delta \rho_{x x} \sim \sum_{r=1}^{\infty} r^{-1} \exp (-2 \pi r \gamma) \cos \left(2 \pi r \omega_{0} / \omega_{c}\right)
$$

where $\gamma$ is the theoretical damping factor related to the level width by $\gamma=\Gamma / \hbar \omega_{c}$. For $2 \pi \gamma>1$ the $\mathrm{r}=1$ term dominates and the series collapses to the empirical form Eqn. 2.18, allowing a correspondence to be drawn between the theoretical and experimental damping factors:

$$
\tilde{\gamma}=2 \pi \gamma \frac{\omega_{c}}{\omega_{0}}=\frac{2 \pi \Gamma}{\hbar \omega_{0}}
$$

Thus the empirical damping factor will depend on magnetic field in the same way as the level width. This is only strictly true for Lorentzian broadening and although other shapes of the density of states lead to equations similar to Eqn. 2.19, the damping factor does not obey Eqn. 2.20 and becomes difficult to calculate.

Barker noticed that the major contribution to $\sigma_{\mathrm{xx}}$ came from processes involving small momentum transfer along the magnetic field, so the $q_{z}$ dependence of the interactions could be neglected. Thus, to a first approximation, the results should also hold for two-dimensional electrons interacting with $3-D$ phonons. Lassnig \& Zawadzki (1983) performed calculations in 2-D starting from the Kubo formula adapted for inelastic optic phonon scattering:

$$
\begin{array}{r}
\sigma_{x x}=\frac{e^{2}}{2 k T} \int_{-\infty}^{\infty} d E \sum_{-q} \frac{2 \pi}{\hbar}\left[l^{2} q_{y}\right]^{2} C(q)^{2} N_{q}\left(N_{q}+1\right)\left[f\left(E-\hbar \omega_{0}\right)-f(E)\right] \\
\times \operatorname{Tr}\left[\delta\left(E-H_{0}\right) \exp (i q . r) \delta\left(E-\hbar \omega_{0}-H_{0}\right) \exp (-i q . r)\right]
\end{array}
$$

where $N_{q}$ is the distribution function for optic phonons of energy $\hbar \omega_{0}, H_{0}$ is the 
unperturbed electron Hamiltonian and $|C(q)|^{2}$ is the Fröhlich interaction potential given by the expectation value of the last term in Eqn. 1.28. In 2-D there are delta function discontiuities at the resonance condition which are removed by replacing the delta functions with Lorentzian spectral densities of width $\Gamma$. Physically this is due to scattering of electrons by processes other than polar optic phonon, but in the calculations only short range scattering has been assumed. The electron wavefuncions are treated by the Fang-Howard variational approach (Eqn. 1.8) and the calculations performed numerically. The results agree with the oscillatory part of the measured resistance and also predict a non-oscillatory contribution from electron-optic phonon scattering of $\sim 20 \%$ of the normalised resistance.

From similar calculations Mori et al. (1988a) have found analytic expressions for Lorentzian, Gaussian and elliptic densities of states. For Lorentzian broadening the oscillatory part of the transverse magnetoresistance is:

$$
\frac{\Delta \rho_{x x}}{\rho_{0}}=2 \overline{\Delta \rho} \sum_{\mathrm{r}=1}^{\infty} \exp (-2 \pi \mathrm{r} \gamma) \cos \left(2 \pi \mathrm{r} \omega_{0} / \omega_{\mathrm{c}}\right)
$$

where the major difference from the bulk case of Eqn. 2.19 is the omission of the $1 / \mathrm{r}$ prefactor, and the pre-exponential which is given by:

$$
\overline{\Delta \rho}=\frac{3}{32}\left[1+\frac{1}{3} \frac{\hbar^{2} b^{2}}{2 m} \frac{1}{\hbar \omega_{0}}\right]^{-1} \mathrm{~N}_{0}\left(\mathrm{~N}_{0}+1\right) \alpha \frac{b \omega_{0}^{2} \tau}{\mathrm{n}_{\mathrm{s}} \mathrm{kT}} \sqrt{ }\left(2 \hbar \omega_{\mathrm{o}} \mathrm{m}^{*}\right) \Theta
$$

where $b$ is the Fang-Howard variational parameter (Eqn. 1.10), $\alpha$ is the Froblich coupling constant (Eqn. 1.29)

and $\theta=\frac{\omega_{c}}{\omega_{0}} \sum_{N}\left\{f\left(E_{N}\right)-f\left(E_{N}+\hbar \omega_{0}\right)\right\}, \quad$ almost independent of magnetic field, with $f\left(E_{N}\right)$ being the Fermi-Dirac distribution function for the $N^{\text {th }}$ Landau level. The second term in the square brackets looks like $1 / 3\left\langle\mathrm{~T}_{\mathrm{Z}}\right\rangle$ in units of the optic phonon energy and so will usually be $<<1$. This leaves $\overline{\Delta p} \propto n_{s}^{-2 / 3}$, field independent and with a temperature dependence fixed by the phonon occupation number. Further since $\rho_{0} \approx 1 / \mathrm{n}_{\mathrm{s}} \mathrm{e} \mu$ and $\mu$ is optic phonon limited at high temperatures, $\Delta \rho_{\mathrm{xx}}$ should decrease rapidly with increasing carrier concentration. 
From the same theory Hamaguchi et al. (1988) find the non-oscillatory part of the magnetoresistance to be given by

$$
\rho_{\mathrm{B}}=\rho_{0}+2 \overline{\Delta \rho}[1 / \pi] \tan ^{-1}[1 / \gamma]
$$

ie. the background magnetoresistance should show a similar variation between samples as the magnetophonon resonance amplitude. These predictions will be discussed in the light of the experimental results of Chapter 3.

By contrast, Warmenbol et al. (1988) used the momentum balance equation 2.6 , and considered a set of Gaussian broadened Landau levels superimposed on a flat background [Section 1.5 considered the experimental evidence for such a model]. Taking account of phonon absorbtion and emission the driving force becomes:

$$
\begin{aligned}
F(v)=\frac{1}{n e} \sum_{-q} \frac{q}{\hbar}\left|V_{q}\right|^{2} & \int_{-\infty}^{\infty} d t \quad\left\{\left[N_{q}+1\right] \exp \left\{i t\left(\omega_{q}-q \cdot v\right)\right\}-N_{q} \exp \left\{-i t\left(\omega_{q}-q \cdot v\right)\right\}\right\} \\
\times & \operatorname{Tr}[f(H) \exp \{-i q \cdot r(0)\}[1-f(H)] \exp \{-i q \cdot r(t)\}]
\end{aligned}
$$

where the trace is of the Fourier transform of the electron density-density correlation function. In the linear regime $\mathbf{v} \rightarrow 0$ and the results are identical to those from the Kubo formula at high fields. The non-linear regime, which corresponds to the case of HEMPR, is more complicated and will be left until Chapter 5.

In the absence of the background the oscillatory part of the resistivity is given by

$$
\begin{aligned}
\Delta \rho_{\mathbf{x x}}=\sqrt{\pi} & \frac{\alpha \beta}{\Gamma} \frac{\omega_{\mathrm{c}}}{\omega_{0}} \mathrm{n}\left(\omega_{0}\right) \quad\left[1-\exp \left(-\beta \hbar \omega_{\mathrm{c}}\right)\right] \\
& \times \Gamma_{\mathbf{n}, \mathrm{m}} \mathrm{I}_{\mathrm{x}}^{\mathrm{n}, \mathrm{m}} \exp \left[-\left[\frac{(\mathrm{n}-\mathrm{m}) \hbar \omega_{\mathrm{c}}-\hbar \omega_{0}}{\Gamma}\right]+\frac{\hbar^{2} \omega_{0}}{2 \mathrm{kT}}\left[\omega_{0}-(\mathrm{n}+\mathrm{m}) \omega_{\mathrm{c}}\right]-\frac{(\beta \Gamma)^{2}}{16}\right]
\end{aligned}
$$

where $\quad I_{x}^{n, m}=\int_{0}^{\infty} J_{n, m}(x) \exists(x / a) x^{\frac{1}{2}} d x$

and $\exists(x / a)$ is a form factor equal to unity for ideal $2-D$ behaviour. Notice that the last term in Eqn. 2.26 will cause a significant shift of the magnetophonon resonance peak if $\Gamma$ is not $\ll \mathrm{kT}$. Inclusion of the flat background exagerates this shift and reduces the amplitude of the oscillatory part by increasing $\rho_{\mathbf{x x}}$ appreciably between the resonace maxima. 


\subsection{CYCLOTRON RESONANCE}

\subsubsection{Theory}

Electrons may also be excited between Landau levels by photons of electromagnetic radiation. This is known as cyclotron resonance, semi-classically the electrons can be thought to move from one cyclotron orbit to the next higher in energy whenever the incident photon energy equals the cyclotron energy $\hbar \omega_{c}$, which leads to the resonance condition:

$$
\omega=\omega_{c}=\frac{\mathrm{eB}}{\mathrm{m}^{*}}
$$

Thus cyclotron resonance could be detected either by varying the frequency of radiation at a fixed magnetic field or, as used in this case, by using fixed frequency laser radiation and sweeping the magnetic field. For most semiconductors far infra red (FIR) radiation and magnetic fields $2 \sim 10 \mathrm{~T}$ are suitable to observe the resonances. Figure 2.4 illustrates a typical trace for a GaAs-GaAlAs heterojunction.

Detection of the resonance position allows an effective mass to be deduced for the electrons, and it is for this purpose that cyclotron resonance measurements were made in this study. Before this cyclotron resonance mass can be used for any calculations involving magnetophonon resonance it needs to be altered to take account of the weighting of the different transitions occuring in the two resonance phenomena; to allow for the non-parabolicity of the conduction band; and to include polaron contributions. This will be discussed again in Section 3.4.

Strictly cyclotron resonance measures discontinuities in the dynamical conductivity:

$$
\sigma_{ \pm}(\omega)=\frac{\text { in }_{s} e^{2 / m^{*}}}{\omega \pm \omega_{c}-\Sigma(\omega)}
$$

where $\Sigma(\omega)$ is the memory function (Van Royen et al. 1984, Wu et al. 1987), which includes polaron coupling matrix elements summed over initial and final electron states (making it filling factor dependent) and varies with temperature through the phonon occupation number. The effective mass in Eqn. 2.28 includes corrections for band non-parobolicity. The magneto-optical absorbtion spectrum is then proportional to: 


$$
\frac{-\operatorname{Im} \Sigma(\omega)}{\left[\omega-\omega_{c}-\operatorname{Re} \Sigma(\omega)\right]^{2}+[\operatorname{Im} \Sigma(\omega)]^{2}}
$$

which gives peaks in the optical absorbtion when

$$
\omega-\omega_{\mathrm{c}}-\operatorname{Re} \Sigma(\omega)=0 .
$$

This non-linear equation must be solved self consistently. It shows the resonance position will be shifted by polaron coupling. Far from the condition $\omega=\omega_{\text {LO }}$ this shift will be small and can be treated pertabatively. The peak width is given by $\operatorname{Im} \Sigma(\omega)$.

Study of the cyclotron resonance linewidth, $\Gamma_{\mathrm{CR}}$, can give information regarding the level broadenings and the dominant scattering processes. For short range scattering Ando \& Uemura (1974) find the linewidth is related to the mobility by:

$$
\Gamma_{\mathrm{CR}}=\left[\frac{2 \mathrm{~B}_{0}}{\pi \mu}\right]^{\frac{1}{2}}
$$

ie. is proportional to $\mathrm{J}$, in contrast to classical theory which predicts no change in magnetic field. Unscreened ionized impurity scattering should give a field-independent linewidth, but inclusion of screening, which removes the long range contributions at low temperature, results in a similar $\sqrt{ } B$ behaviour. As the polaron region is approached the linewidth will broaden rapidly due to the increase in scattering [and $\operatorname{Im} \Sigma(\omega)$ ]. Other mechanisms responsible for maxima in the linewidth include coupling at the subband energy, magnetoplasmon modes and the filling factor dependence of the screening.

The cyclotron resonance linewidth is often used to put in a value for broadening in magnetotransport calculations, however great care must be excercised. Since cyclotron resonance is a local transition only homogeneous broadening will be important and as such much larger values of mobility are deduced from $\Gamma_{\mathrm{CR}}$ than the d.c. transport mobility, which is sensetive to all broadeing mechanisms. For example Hopkins et al. (1989) report mobilities of $\sim 10^{9} \mathrm{~V}^{-1} \mathrm{~s}^{-1} \mathrm{~cm}^{2}$ in a sample (G139) with a d.c. mobility less than $10^{6} \mathrm{~V}^{-1} \mathrm{~s}^{-1} \mathrm{~cm}^{2}$. Comparisons with the magnetophonon resonance broadening are even more dangerous, since this phenomenum is always observed under resonant polaron coupling conditions where cyclotron resonance could not be performed (in the Reststralen). 


\section{$\underline{\mathbf{2 . 4}} \quad$ Experiment}

The FIR used in the cyclotron resonance experiments was generated by a molecular gas laser, pumped by an Edinburgh Instruments PL4, grating tunable, $\mathrm{CO}_{2}$ laser. By using a variety of molecular gases in the FIR cavity, such a laser system can produce large numbers of lines with wavelengths in the range $35 \mu \mathrm{m}$ to $1200 \mu \mathrm{m}$ (1 to $35 \mathrm{meV}$ ). The optically pumped FIR laser system was first described by Chang \& Bridges (1970) and to date thousands of lines, generated by some tens of different gases, have been obtained (Knight 1982). For these measurements methanol was principally used, and especially the line at $118.83 \mu \mathrm{m}$. The wavelengths of lines used were measured with a Michelson interferometer to an accuracy of $\approx 0.1 \%$.

The resonances were detected from the increased attenuation of the radiation transmitted through the sample by a Ge bolometer, positioned away from the magnet (being field sensetive) and cooled to $\approx 4.2 \mathrm{~K}$ to enhance its efficiency. In order to provide a reference signal for the bolometer response the FIR radiation was modulated by chopping the $\mathrm{CO}_{2}$ laser output at approx. $20 \mathrm{~Hz}$ and the detector voltage fed into a phase sensitive detector referenced to the chopper. The time constants used for signal integration led to a small shift in the resonance position and to correct for this the peak position was averaged from sweeps up and down in field. The resonance is usually observed superimposed on a constant background and its position is taken as the minimum in transmission. To avoid distortion due to interference effects from multiple internal reflections the sample substrate was polished to form a wedge of $\approx 2^{\circ}$. Further details of cyclotron resonance experiments and the operation of the laser system are given by Hopkins (1987) and by Howell (1989). 
Figure 2.4 Typical experimental recording of cyclotron resonance in a GaAs-GaAlAs heterojunction at $1.6 \mathrm{~K}$. The insert shows an expansion of the resonance.

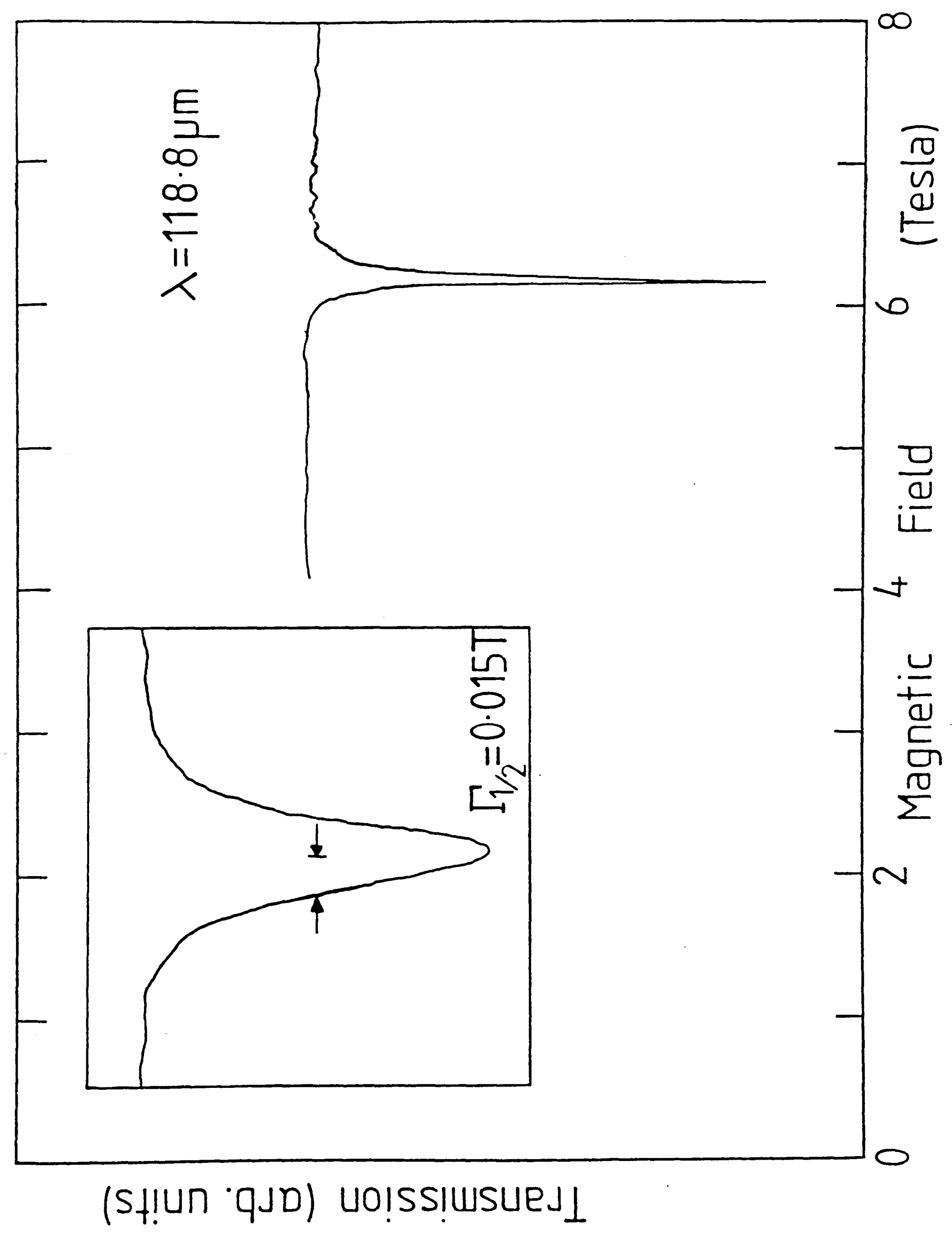




\subsection{THE MAGNETS}

The high magnetic field system at the Clarendon Laboratory is based around a 2.2MW motor generator set used to energise a number of air (room temperature)-bore solenoid magnets to $4800 \mathrm{~A}$ at $450 \mathrm{~V}$ d.c. The generator current is controlled to within a few parts in $10^{5}$ (Smith 1967), and the field is calculated from a shunt voltage. Most of the experiments were performed using $50 \mathrm{~mm}$ bore, resistive solenoids constructed on the Tsai pattern (flat, copper wound pancakes) and capable of producing magnetic fields of just over $9 \mathrm{~T}$ (Hudson 1972, Hudson \& Jones 1981). The field centre and calibration were determined using the NMR signal of protons and fluorine nuclei; the calibration is accurate to $0.1 \%$, and the homogeneity at the field centre is better than $0.5 \%$ over a sphere of $2 \mathrm{~cm}$ diameter. Over the life of the magnets the calibration has changed slightly due to degradation of the epoxy resin used to insulate the coils. The magnet coils are cooled by a flow of $2 \mathrm{~m}^{3} /$ minute of deionised water, circulated from a tank on the roof by an 80 h.p. pump. During magnet operation the water temperature is kept below $30^{\circ} \mathrm{C}$ by a forced blast cooling tower with a cooling power of $1 \mathrm{MW}$.

Magnetotransport experiments have also been performed using the hybrid magnet (Hudson \& Jones 1981) which is a coaxial arrangements of two coils: the outer coil is a superconductor producing a central field of $6.5 \mathrm{~T}$ in a $245 \mathrm{~mm}$ bore; while the inner is a water cooled solenoid of the polyhelix design (Hudson et al. 1984, Jones \& Whitworth 1986), producing an additional field of $10 \mathrm{~T}$ in a $50 \mathrm{~mm}$ bore or $13.5 \mathrm{~T}$ in a $30 \mathrm{~mm}$ bore, giving combined fields of $16 \frac{1}{2}$ and $20 \mathrm{~T}$ respectively. The hybrid system thus enables rapid sweeps of the magnetic field over a 10 to $13 \mathrm{~T}$ range up to the combined field limit of $20 \mathrm{~T}$.

The principle advantages of using these resistive magnets, as opposed to state-of-the-art superconductors, is in the speed with which the field can be swept. This was particularly useful when taking differentiated traces (see below) and when rotating the samples in field allowed a large number of data points to be assimilated in a reasonable time. 


\section{$\underline{2.6}$ CRYOGENICS}

Nearly all these experiments were performed below room temperature. Three cryogenic arrangements were used:

(i) Bath Cryostat - A standard ${ }^{4} \mathrm{He}$ bath cryostat, capable of fitting in the $50 \mathrm{~mm}$ bore magnet, was use for transport measurements between $4.2 \mathrm{~K}$ and $1.5 \mathrm{~K}$. This cryostat was also used for magnetophonon measurements: suitable cooling from liquid nitrogen (which was not allowed to condense) allowed experiments to be performed at reasonably stable temperatures between $120 \mathrm{~K}$ and $300 \mathrm{~K}$; liquid nitrogen at reduced pressure was used for some hot electron magnetoresonance experiments between $77 \mathrm{~K}$ and 50K. In this set up thermometry was performed using the vapour pressure of liquid helium, or nitrogen. At higher temperatures a semiconductor diode, mounted close to the sample, was used: with a constant current of $10 \mu \mathrm{A}$ the forward voltage changed linearly from $0.53 \mathrm{~V}$ at $300 \mathrm{~K}$ to $1.10 \mathrm{~V}$ at $77 \mathrm{~K}$.

(ii) Flow Cryostat - An Oxford Instuments CF1200 continuous-flow cryostat was used between $300 \mathrm{~K}$ and $4.2 \mathrm{~K}$ for magnetophonon and cyclotron resonance experiments. This was of a smaller bore and so fitted in the $30 \mathrm{~mm}$ bore hybrid magnet. The temperature was altered by balancing the flow rate of ${ }^{4} \mathrm{He}$ and power supplied to a heater. Thermometry was by thermocouple, conected to the flow controller, and temperatures compared favourably with those deduced from the diode.

(iii) $500 \mathrm{mK}{ }^{3} \mathrm{He}$ System - Use of this cryostat, which is top loaded into a standard ${ }^{4} \mathrm{He}$ bath cryostat, allowed magnetoresistance measurements to be made between $500 \mathrm{mK}$ and 4.2K. Details of the design are given by Usher (1988). Being designed to fit in the $30 \mathrm{~mm}$ bore magnets, unlike the other two system, there was not space for a sample rotation mechanism inside the insert. Temperature measurement was by germanium resistance thermometer, calibrated between $300 \mathrm{mK}$ and $6 \mathrm{~K}$ by Lake Shore Cryogenics, in conjunction with ${ }^{3} \mathrm{He}$ vapour pressure. The $\mathrm{Ge}$ resistor, although accurate and reproducible, was affected by the magnetic field and so temperature had to be measured in zero field. Care had to be taken in sweeping the magnet, as sweep rates greater than $80 \mathrm{mT} / \mathrm{s}$ tended to warm the system, as did excessive use of an LED. 


\subsection{ELECTRICAL MEASUREMENTS}

\subsubsection{Experimental Arrangement}

Most of the experiments reported in this thesis involve measuring the resistivity of samples under various conditions. Wherever possible four contact measurements were made, which avoids measuring parasitic contact resistances. The usual method was to apply a constant current $(0.1-100 \mu \mathrm{A})$ to the ends of a long, thin bar of material and record the voltage produced between probes along the sides of the bar as a function of magnetic field, on an $x-y$ recorder. In some cases it was found that the input impedence of the chart recorder had an effect on the signal (ie. was too small), and so a Keithley 147 buffer amplifier was used.

Use of d.c. was found to be unsuitable for the very low temperature Shubnikov-de Haas measurements described in Chapters $6 \& 8$, as there was either excessive heating of the electron gas or a poor signal-to-noise ratio. Consequently in these experiments an a.c. system was used with the signal being detected at the frequency of the current using a lock-in amplifier. This allowed suitable recordings to be taken at currents as small as $10 \mathrm{nA}$, at a frequency of $\sim 17 \mathrm{~Hz}$.

\section{Sample Geometry}

The samples used were either cut into bars approx. $2 \times 10 \mathrm{~mm}$ using a diamond saw, or were supplied with a Hall bar defined by photolithography, as shown in Fig. 2.5. The Hall bars supplied by Philips were fabricated from three mask shapes and came in two sizes $-2.5 \mathrm{~mm}$ and $5 \mathrm{~mm}$ squares, with both sized samples having the same aspect ratio. The dimensions of each type of bar are given in Table 2.1.

\begin{tabular}{|c|c|c|c|c|c|}
\hline Mask \# & \multicolumn{2}{|c|}{$\begin{array}{c}\text { Large }(5 \mathrm{~mm} \\
\mathrm{w}(\mu \mathrm{q})\end{array}$} & \multicolumn{2}{|c|}{$\begin{array}{c}\text { Sma11 } \\
\mathrm{w} \\
(2.5 \mathrm{~mm}) \mathrm{sq})\end{array}$} & $\frac{l}{\mathrm{w}}$ \\
\hline 1 & 50 & 758 & 25 & 379 & 30.33 \\
\hline 2 & 100 & 767 & 50 & 383 & 15.33 \\
\hline 3 & 150 & 775 & 75 & 388 & 10.33 \\
\hline
\end{tabular}

\section{Table 2.1}




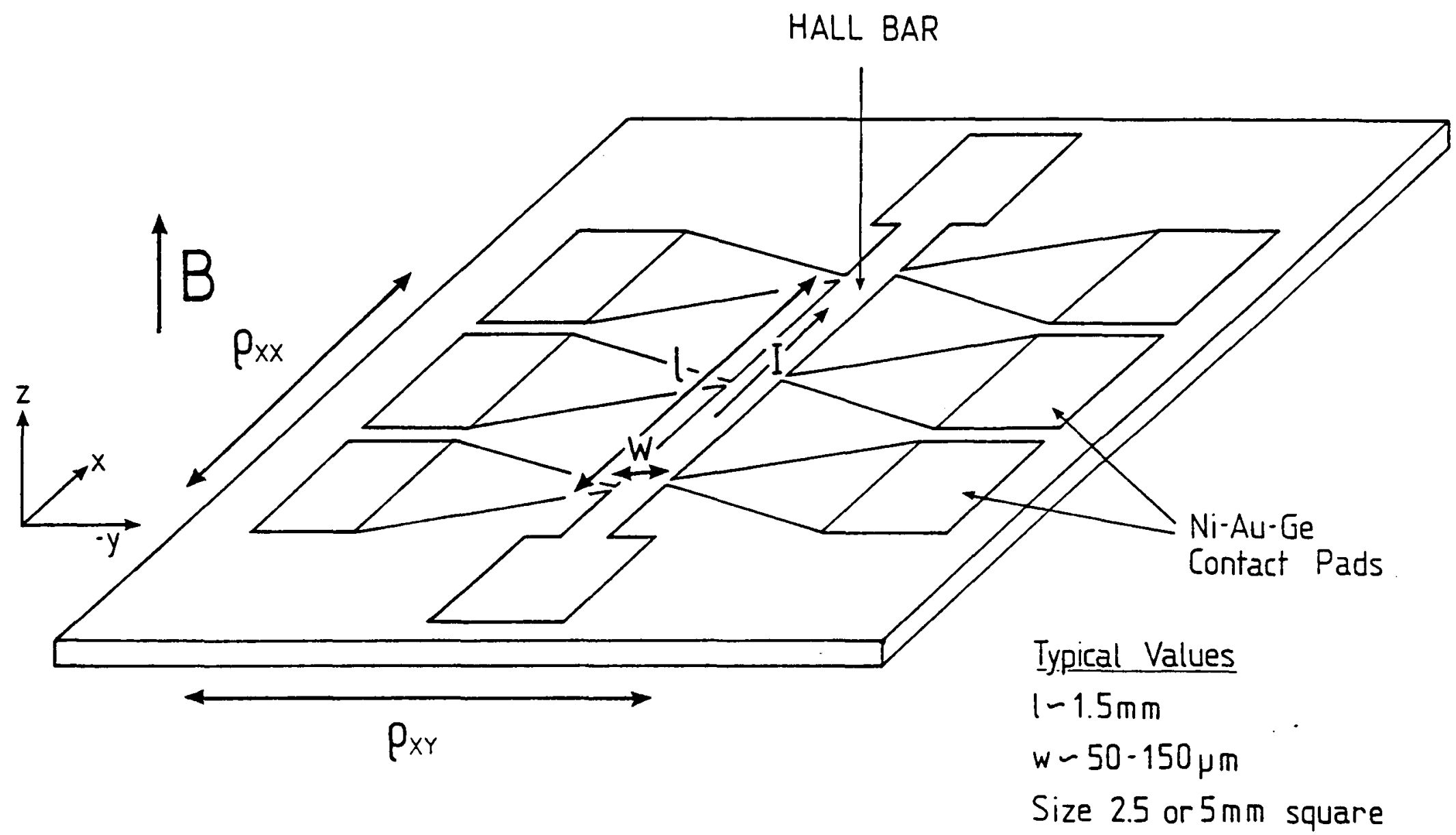

Figure 2.5 Lithographed Hall Bar Sample.

In each case current contacts were provided at either end of the bar together with three pairs of voltage contacts along the bar. These enabled the sheet carrier density, $\mathrm{n}_{\mathrm{s}}$, and mobility, $\mu$, to be easily determined:

$$
\mathrm{n}_{\mathrm{s}}=\frac{I}{\mathrm{eV}_{\mathrm{H}}}
$$

where $I$ is the current flowing along the bar and $\mathrm{V}_{\mathrm{H}}$ is the Hall voltage per unit magnetic field measured between any pair of opposite contacts:

$$
\mu=\frac{I}{\mathrm{n}_{\mathrm{s}} \mathrm{eV}} \frac{l}{\mathrm{w}}
$$

where $V_{0}$ is the zero field voltage along the bar. By making simultaneous measurements of $V_{O}$ and $V_{H}$ the current and carrier density can be eliminated in measuring the mobility:

$$
\mu=\overrightarrow{\mathrm{V}}_{\mathrm{O}} \frac{l}{\mathrm{w}}
$$




\section{Electrical Contacts}

The fabrication of reliable ohmic contacts is very important and extremely difficult. The lithographed GaAs-GaAlAs bars had Ni-Au-Ge contacts diffused into the 2DEG by the sample growers at Philips. The Ge dopes the contact region heavily n-type, then the $\mathrm{Ni}$ acts as a wetting agent for the $\mathrm{Au}$, which replaces atoms on the $\mathrm{Ga}$ sites. Since the diffusion depth in GaAlAs is $\sim 2000 \AA$, this contacting technique becomes unreliable for samples with spacer layers of this order or greater.

For the other bars electrical contacts were made by alloying indium into the semiconductor. Small pieces of In $(\sim 0.2 \mathrm{~mm}$ diameter $)$ were placed on the cleaned surface of the bar and heated in a reducing $\mathrm{H}_{2}-\mathrm{N}_{2}$ atmosphere. The flowing gas was passed through conc. $\mathrm{HCl}$ for a few seconds at the melting point of $\mathrm{In}\left(\sim 160^{\circ} \mathrm{C}\right)$ and again at the maximum alloying temperature to provide a flux. However excess flux was avoided as this would be liable to etch away at the surface or form $\mathrm{InCl}_{3}$. The alloying temperature used varied with material: for a GaAs-GaAlAs heterojunction $350^{\circ} \mathrm{C}$ for 2 minutes was suitable. Contacts were much easier to alloy into indium containing compounds, but trouble was expirienced with the thick layers of GaAlAs found in the MQWs. This meant that in stacks of 30 wells all the wells were not always contacted to. Tin contacts have also been successfully used (Ploog \& Brummell 1988).

\subsubsection{Detection of Small Oscillations}

The oscillatory effects considered in this thesis (magnetophonon resonances and Shubnikov-de Haas oscillations) often lead to rather small changes in the resisitivity superimposed on a background showing a fairly rapid monotonic increase with magnetic field. This makes analysis from direct recordings of the magnetoresistance difficult, and so to extract these small oscillations two methods have been used:

\section{Compensation}

At high temperatures the background magnetoresistance is approximately linear (Fig. 2.6a) and so one way of extracting the oscillatory structure is by subtraction of a voltage linearly proportional to the magnetic field (Fig. 2.6b). In practice this was 
Figure 2.6 Magnetophonon oscillations from G148 showing (a) direct, (b) compensated and (c) double differentiated trraces.

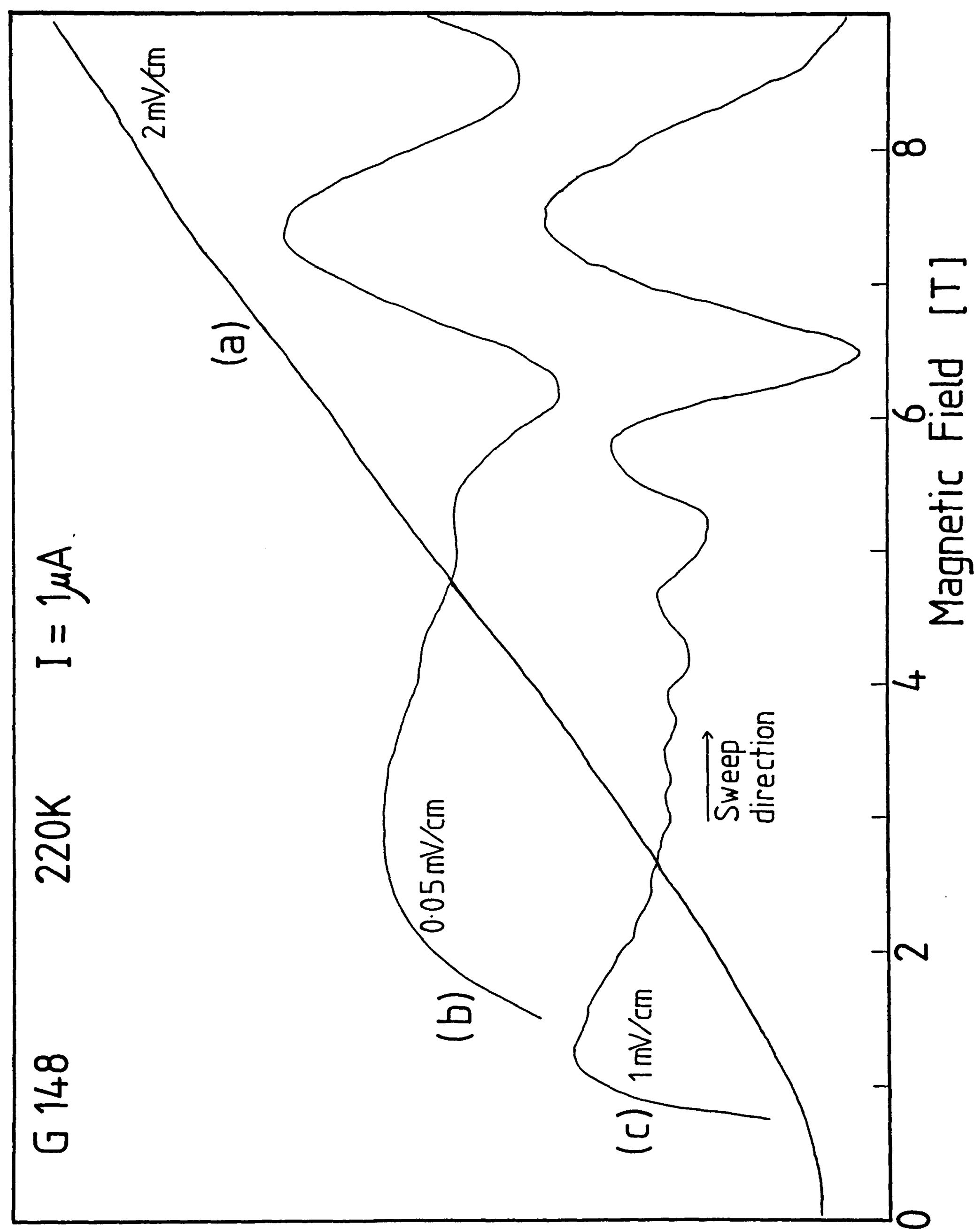


achieved by taking a voltage proportional to the magnet current and optimising the constant of proportionality according to the sample, temperature and magnetic field region of intrest. By this technique oscillations $10^{-4}$ of the background could be resolved, making it ideal for measuring oscillation amplitudes.

At lower temperatures and higher measuring currents (see Chapters $5 \& 8$ for measurements outside the ohmic region) the magnetoresistance becomes highly non-linear and so this technique becomes less useful. In principle a polynomial in $\mathbf{B}$ could be subtracted, but the time required to fit it to the background would be prohibitively long and using a computer algorithm would probably also subtract the oscillations themselves!

At the lowest temperatures used the background magnetoresistance was much smaller and direct traces were often adequet.

\section{Double Differentiation}

An alternative method of suppressing the background is to take the second derivative of the magnetoresistance with respect to magnetic field. This has the effect of enhancing rapidly varying oscillatory components and is particularly useful for finding accurate resonance positions (Fig. 2.6c). There are a number of possible practical methods for taking second derivatives, discussed in detail by Hoult (1974), including magnetic field modulation, usually with additional modulation coils; electronic filtering; and digital processing.

In this work the second method was chosen with the signal beind fed through a pair of high- and low-pass active filters, using a circuit devised by Sallen \& Key in 1955. The high-pass filter has a $12 \mathrm{~dB}$ per octave response proportional to $\omega^{2}$ and so produces the second derivative of a sinusoidal input. The $12 \mathrm{~dB}$ per octave low-pass filter eliminates high frequency noise and amplifies the signal by $26 \mathrm{~dB}$. The cut off frequency could be adjusted between 0.16 and $1.0 \mathrm{~Hz}$, with differentiation occuring for all frequencies below this. Active filters coupled to a d.c. amplifier are prefered to passive components where the large inductors used tend to create hum. By sweeping the magnetic field this filtering is equivalent to a field derivative (and will be refered to as $d^{2} / d \mathrm{~B}^{2}$ rather than $\left.d^{2} / d \mathrm{t}^{2}\right)$, picking out certain ranges of periodicites in $\mathrm{B}$ depending 
on the sweep rate.

For the magnetophonon experiments the required components could be obtained with sweep rates between 50 and $200 \mathrm{mTs}^{-1}$ for the resonances between 3 and $9 \mathrm{~T}$. As the magnetophonon extrema are periodic in $1 / B$, not $B$, they all correspond to a different frequency and so the amplification of each resonance will be different. This means accurate amplitude measurements can only be made at the same magnetic field and sweep rate, although the $\omega^{2}$ response of the filters can be used to make approximate comparisons between different fields or sweep rates. To resolve resonances of higher harmonic number, at lower. fields, slower sweep rates are required, but this makes the signal more susceptible to noise.

The second derivative technique was also used for the low temperature Shubnikov-de Haas measurements below 1T, described in Chapters $6 \& 8$. In these experiments the harmonic numbers of the oscillations were typically $>20$ and so the response of the filters was less critical providing that a slow enough sweep rate was used - typically $\sim 3 \mathrm{mTs}^{-1}$.

Thus, differentiation allowed much weaker signals to be extracted than was possible by straight subtraction, but had several problems:

(i) The action of taking a second derivative changes the sign of all the oscillation extrema - for this reason all traces will display $-d^{2} \rho / d^{2}$ to give maxima at positions of maxima in the resistivity.

(ii) As well as differentiating the filters produce a phase shift, which can be removed by averaging peak positions from sweeps up and down in magnetic field. This phase shift is frequency dependent and so results in asymmetric distortion of the peaks making absolute measurements of peak amplitude unreliable. Although comparison of peak heights of a given harmonic number is fine, absolute amplitudes must be found by comparison with a suitable direct/compensated trace.

(iii) Transients at the beginning of each sweep limit the useful field range, especially at the higher sweep speeds. Also noise induced transients at the resonant frequency of the filters can look remarkably similar to magnetophonon resonances! 


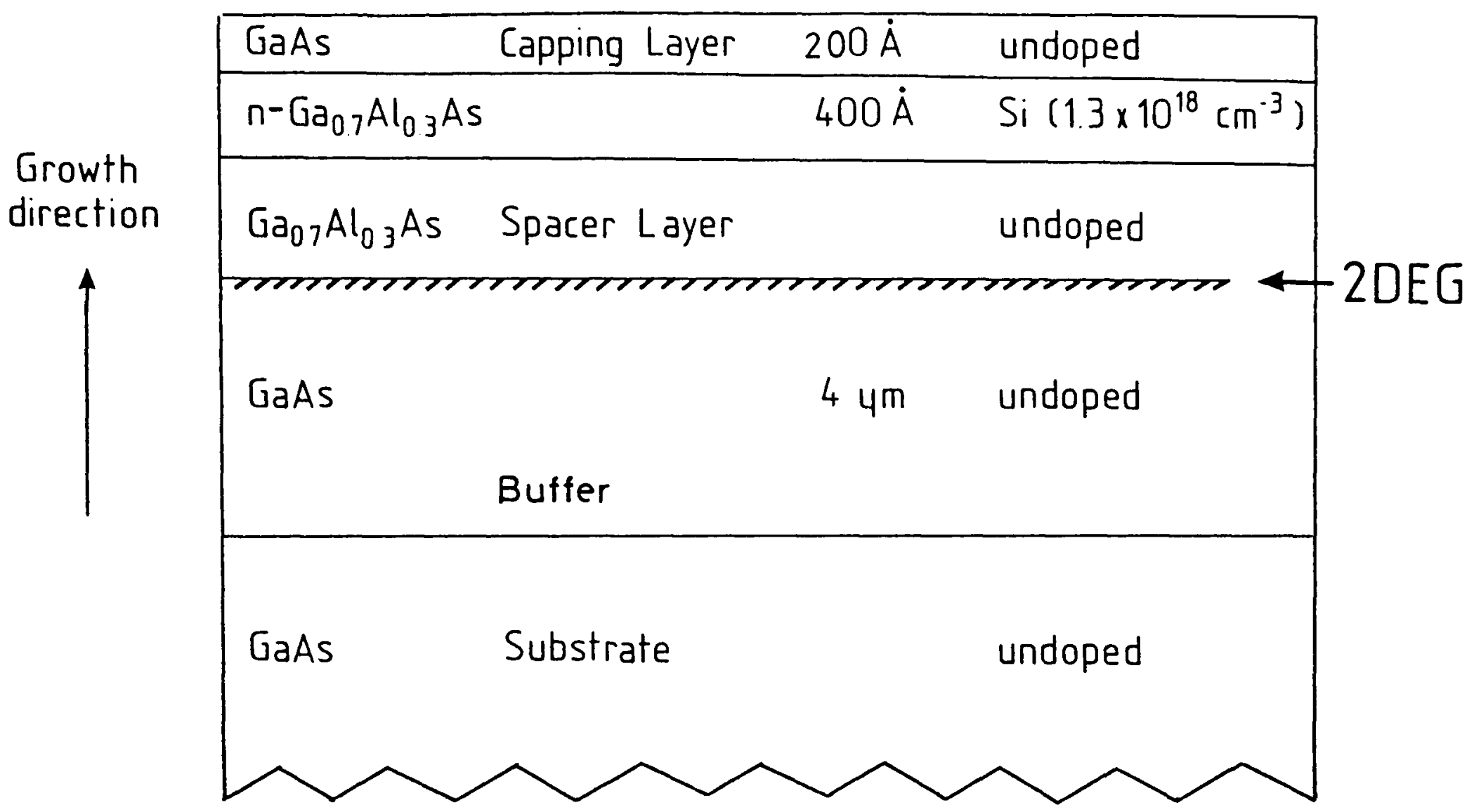

Figure 2.7 Schematic growth profile of a GaAs-GaAlAs heterojunction.

\subsection{SAMPLE PARAMETERS}

\subsubsection{GaAs-GaAlAs Heterojunctions}

The GaAs-GaAlAs heterojunctions used for this work were grown by MBE at Philips Research Laboratories, Redhill, Surrey by Dr. C.T. Foxon (Foxon \& Harris 1986, Harris et al. 1986). They are all conventional modulation doped structures, as depicted schematically in Fig. 2.7. By use of spacer layers beween $17 \AA$ and $3200 \AA$ electron densities in the 2DEG from below $1 \times 10^{10} \mathrm{~cm}^{-2}$ to greater than $1 \times 10^{12} \mathrm{~cm}^{-2}$ were achieved as detailed in Table 2.2. Growth was performed at a steady temperature of $650^{\circ} \mathrm{C}$. The Al fraction in the alloy layers was $32 \pm 1 \%$ so there should be little change between samples in the barrier heights. Similarly the doping density was kept between $1.29 \& 1.41 \times 10^{18} \mathrm{~cm}^{-2}$ in all samples. Samples G156 and G148 had thicker doped regions of $500 \dot{A}$, which led to slightly higher mobilities than found at the same carrier density in otherwise identical samples.

Most samples had a closely compensated p-type buffer layer, with a background

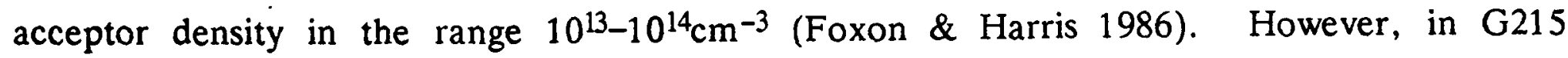
the buffer layer was unintentionally n-type - giving G215 a quasi-accumulation layer appearance and a larger carrier density than expected for a $200 \AA$ spacer layer sample. 


\begin{tabular}{|c|c|c|c|}
\hline \multicolumn{2}{|c|}{ 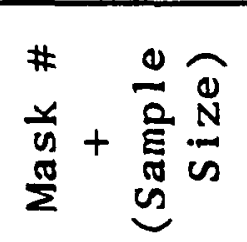 } & 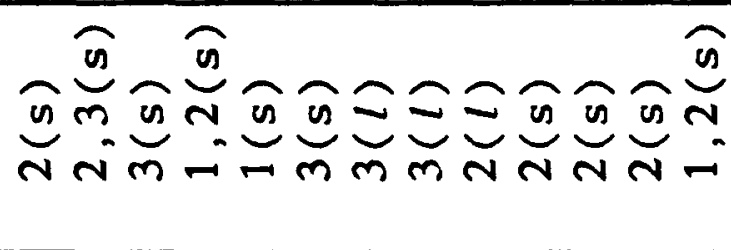 & \multirow{11}{*}{ 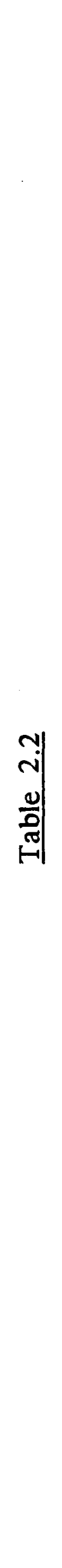 } \\
\hline \multirow{2}{*}{$\underset{\Xi}{N_{a}^{i}}$} & 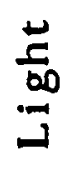 & 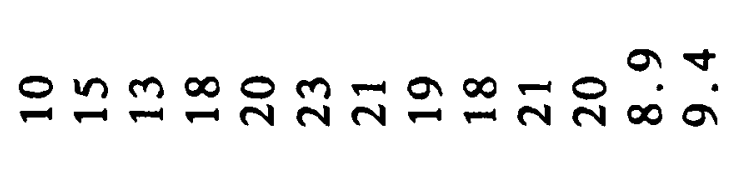 & \\
\hline & $\frac{\ddot{L}}{\underline{a}}$ & 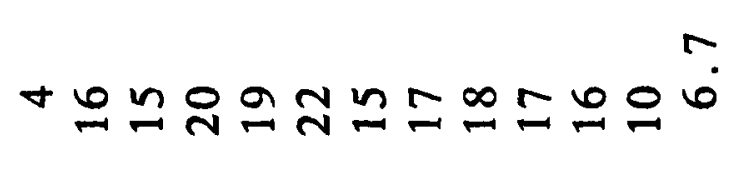 & \\
\hline \multirow{2}{*}{ 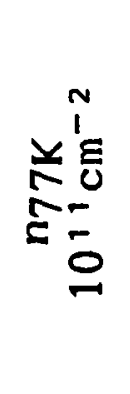 } & 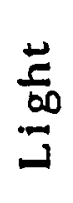 & 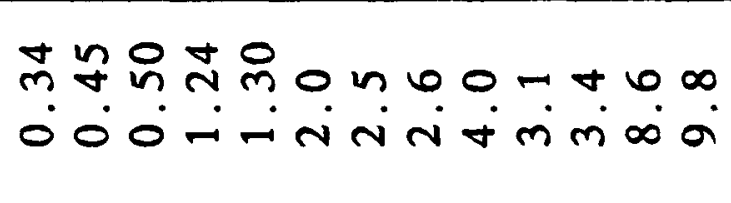 & \\
\hline & 光 & 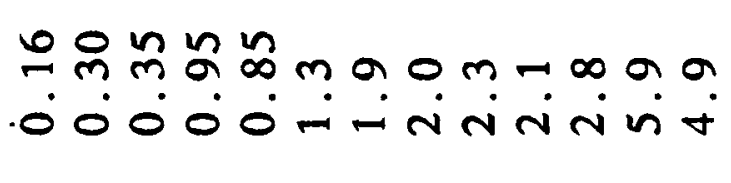 & \\
\hline${ }^{i}=$ & $\frac{\overrightarrow{5}}{a}$ & 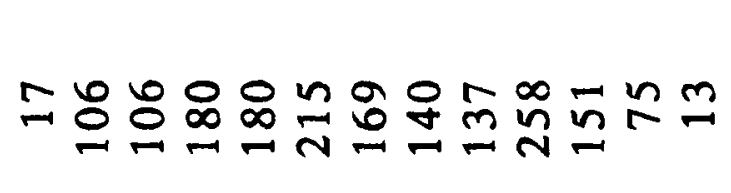 & \\
\hline N & 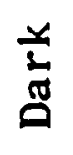 & । m & \\
\hline 将 & $\begin{array}{l}\stackrel{\overrightarrow{5}}{\infty} \\
\vec{J}\end{array}$ & 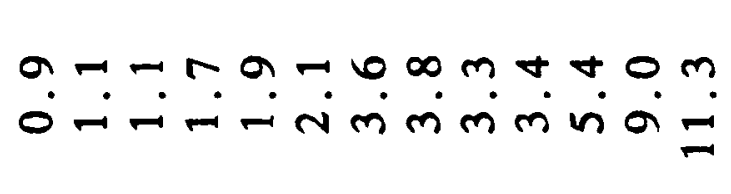 & \\
\hline 0 & 竞 & I & \\
\hline \multicolumn{2}{|c|}{ 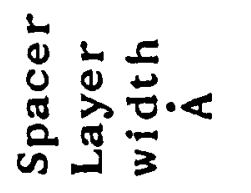 } & 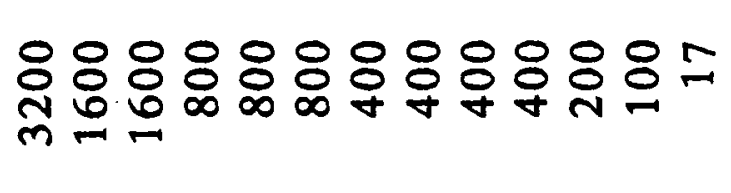 & \\
\hline \multicolumn{2}{|c|}{$\frac{\omega}{2}$} & 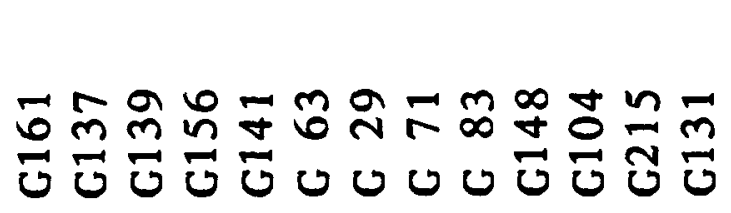 & \\
\hline
\end{tabular}


It also had a larger mobility than expected for its carrier density, due to fewer ionised impurities in the GaAlAs.

Sample G161 was also slightly strange: the sheet electron density of only $1 \times 10^{10} \mathrm{~cm}^{-2}$ is comperable to the background acceptor density and consequently G161 showed mixed conduction. At this carrier density the average in plane distance centres of the electron wavefunctions is $\sim 1000 \AA$, which is comparable to, or larger than, the expected extent of the said wavefunction. This makes the oveerlap between adjacent states small and so means the sample resistance is unusually high in the dark. Making electrical contact to this sample was also a problem as the contacts had to penetrate $3600 \AA$ of $\mathrm{GaAlAs}$ to reach the $2 \mathrm{DEG}$. In practice reproducible results were only obtained after the electron density had been increased to $\sim 3 \times 10^{10}$ by illumination.

The parameters that best describe the samples are the zero field mobility, in the dark and after illumination with a red LED, and the corresponding carrier densities at $4 \mathrm{~K}$ and $77 \mathrm{~K}$. These are tabulated in Table 2.2. Further measurements and comments on the sample mobilities at low temperatures will be made in Chapter 7 . At $\sim 150 \mathrm{~K}$ the mobility is phonon limited and is approximately constant in all the samples, as shown in Fig. 2.8 .

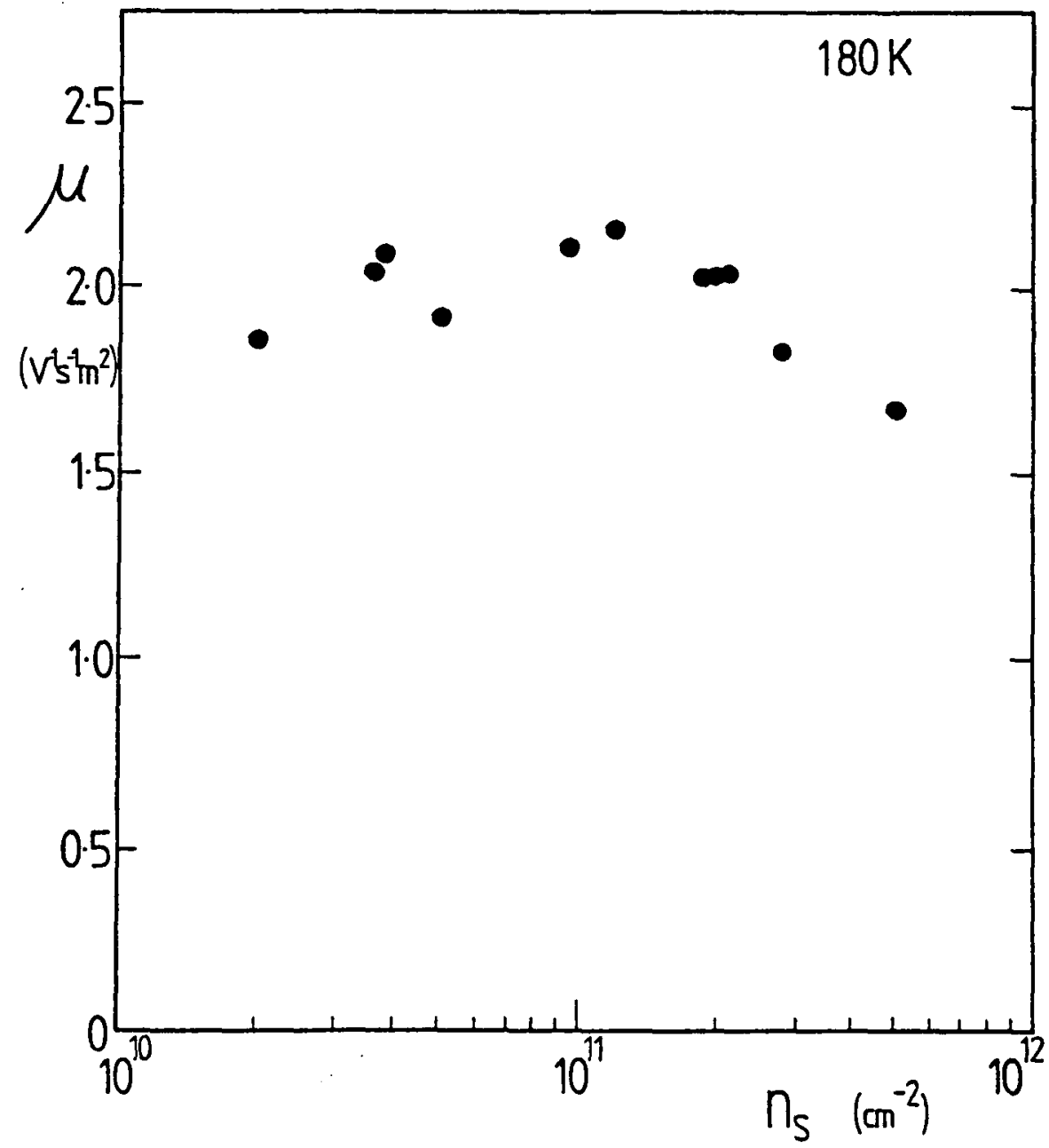

Figure 2.8 Change in GaAs-GaAlAs heterojunction mobility with carrier concentration. 


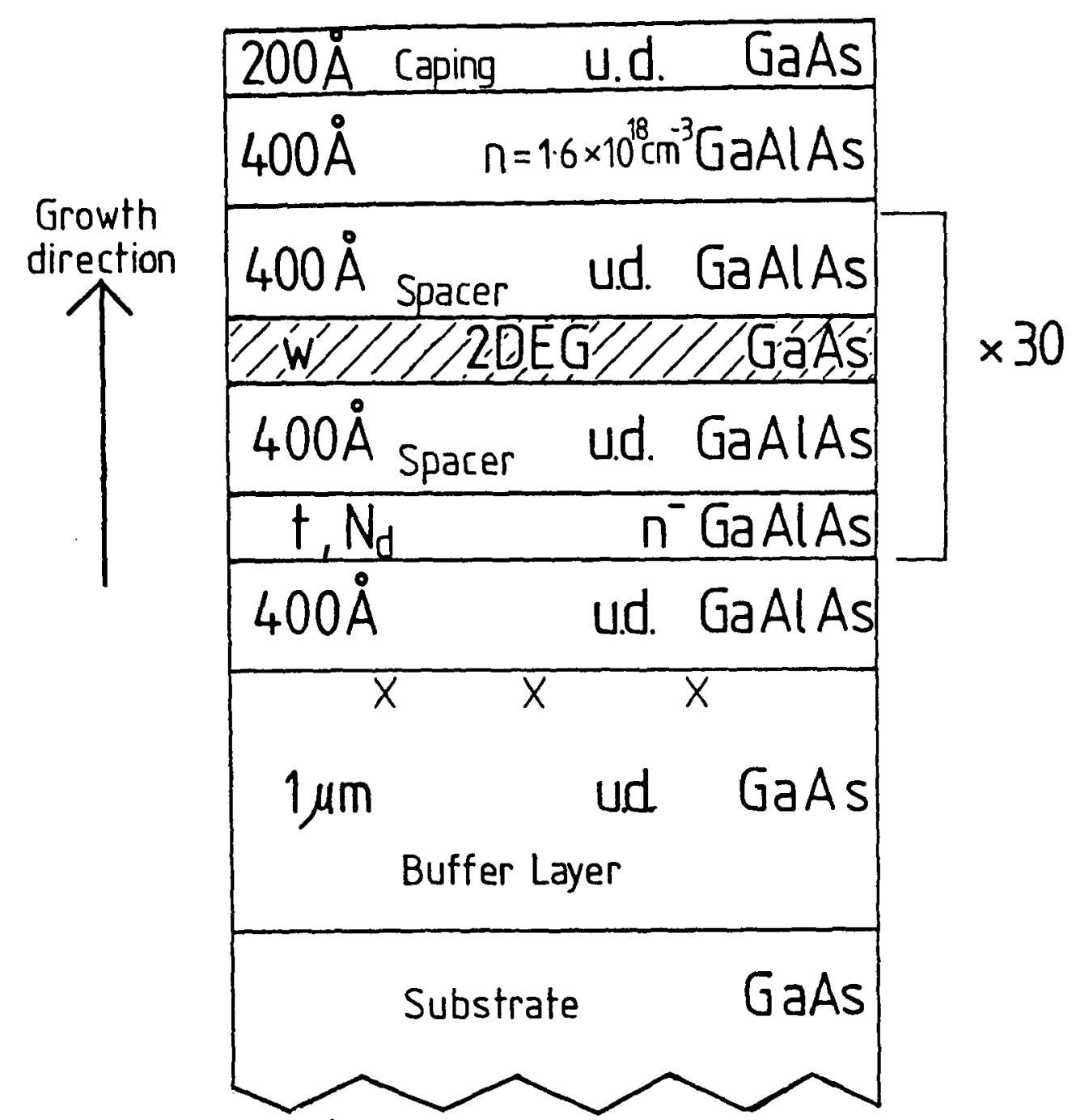

Figure 2.9 Growth profile of GaAs-GaAlAs Multiple Quantum Well sample.

\subsubsection{GaAs-GaAlAs Quantum Wells}

Multi-quantum wells were also grown by MBE at Philips. In growing quantum wells there is a problem in forming the inverted interface of GaAs on GaAlAs (Drummond et al. 1983). The basic difficulties are: diffusion of the Si dopant upwards towards the interface; a higher interface disorder; and accumulation of $\mathrm{C}$ impurities on the GaAlAs surface - this is why a GaAs caping layer is usually grown on top of heterojunctions.

The samples used consisted of sets of 30 quantum wells with the basic structure shown in Fig. 2.9. The spacer layer thicknesses were $400 \AA$ in all samples, except $G 182$ where they are $200 \AA$. The width of the doped layer and doping densities varied (see Table 2.3) but gave a sheet impurity density of $4 \times 10^{11} \mathrm{~cm}^{-2}$, in all except $\mathrm{G} 182$ where it is $5 \times 10^{10} \mathrm{~cm}^{-2}$. Table 2.3 also gives the carrier densities, deduced from the Hall voltage, $\mathrm{n}_{\mathrm{H}}$ (sensitive to the total number of carriers present) and Shubnikov-de Haas measurements, $\mathrm{n}_{\mathrm{SdH}}$ (just depending on the density per well).

It can be seen that all the wells were only definitely contacted in G172 and G177. For $\mathrm{G} 176 \mathrm{n}_{\mathrm{H}} / \mathrm{n}_{\mathrm{SdH}} \approx 10$, suggesting only 10 wells were contacted, or that the Hall voltage contained a contribution from parallel p-type conduction. G163 and G182 show 
only one or possibly two conduction channels. These could be in the parasitic heterojunction that can form at the base of the structure (marked $X$ in Fig. 2.9) or all be in the top well. This could also become a heterojunction with a $400 \AA$ spacer layer due to the fairly hefty doping at the top of the structure. An electric field between the substrate and the top surface would also upset the distribution of electrons cause all the wells to be on a slope and emptying all the electrons into one or two wells at the end of the stack. In the widest wells band bending will produce approximately triangular potential wells at the interfaces, giving the appearence of pairs of heterojunctions. Another problem encountered was that of making good electrical to all the wells in a MQW structure. In these samples there was $>800 \AA$ of GaAlAs between each GaAs well and so $\mathrm{Ni}-\mathrm{Au}-\mathrm{Ge}$ contacting was found to be unsuitable due to the limited diffusion depth. Instead In dots were used but this again was not entirely satisfactory. In practice it would be much better to study transport in high quality single quantum wells than MQW structures.

\begin{tabular}{|c|c|c|c|c|c|c|}
\hline \multirow[t]{2}{*}{ SAMPLE } & \multirow{2}{*}{$\begin{array}{l}\text { We ll } \\
\text { Width } \\
\quad \dot{A}\end{array}$} & \multirow{2}{*}{$\begin{array}{l}\text { GaAlAs } \\
\% \text { Al }\end{array}$} & Doping & \multirow{2}{*}{$\begin{array}{c}\text { Details } \\
\mathrm{N}_{\mathrm{D}} \\
\mathrm{cm}^{-2}\end{array}$} & \multicolumn{2}{|c|}{$\begin{array}{l}\text { Carrier Density } \\
\quad \times 10^{11} \mathrm{~cm}^{-2}\end{array}$} \\
\hline & & & $\dot{A}$ & & $\mathrm{n}_{\mathrm{SdH}}$ & $\mathrm{n}_{\mathbf{H}}$ \\
\hline G176 & 100 & 20 & 27.8 & $1.6 \times 10^{18}$ & 0.9 & 9 \\
\hline G172 & 200 & 20 & 27.8 & $1.6 \times 10^{18}$ & $1-3$ & 60 \\
\hline G163 & 300 & 20 & 27.8 & $1.6 \times 10^{18}$ & - & 70 \\
\hline G177 & 300 & 33 & 33.3 & $1.3 \times 10^{18}$ & 1.3 & 42 \\
\hline G1 82 & 300 & 33 & 83.3 & $6.7 \times 10^{16}$ & 1.4 & 2.6 \\
\hline
\end{tabular}

Table 2.3

Sample parameters of GaAs-GaAlAs quantum wells.

\subsubsection{GaInAs-InP Quantum Wells}

Dr. S.J. Bass at R.S.R.E., Malvern was responsible for growing the GaInAs-InP quantum wells, by AP-MOCVD at $670^{\circ} \mathrm{C}$ (Bass \& Young 1984, Skolnick et al. 1986a,b). A schematic diagram of a modulation doped well is shown in Fig. 2.10. Undoped wells were also investigated, with the spacer and $n$-type InP layers omitted. The donor background in the InP was $\sim 2 \times 10^{15} \mathrm{~cm}^{-2}$ and the modulation doping was with sulphur at the concentrations shown in Table 2.4. For all the samples studied in detail the 


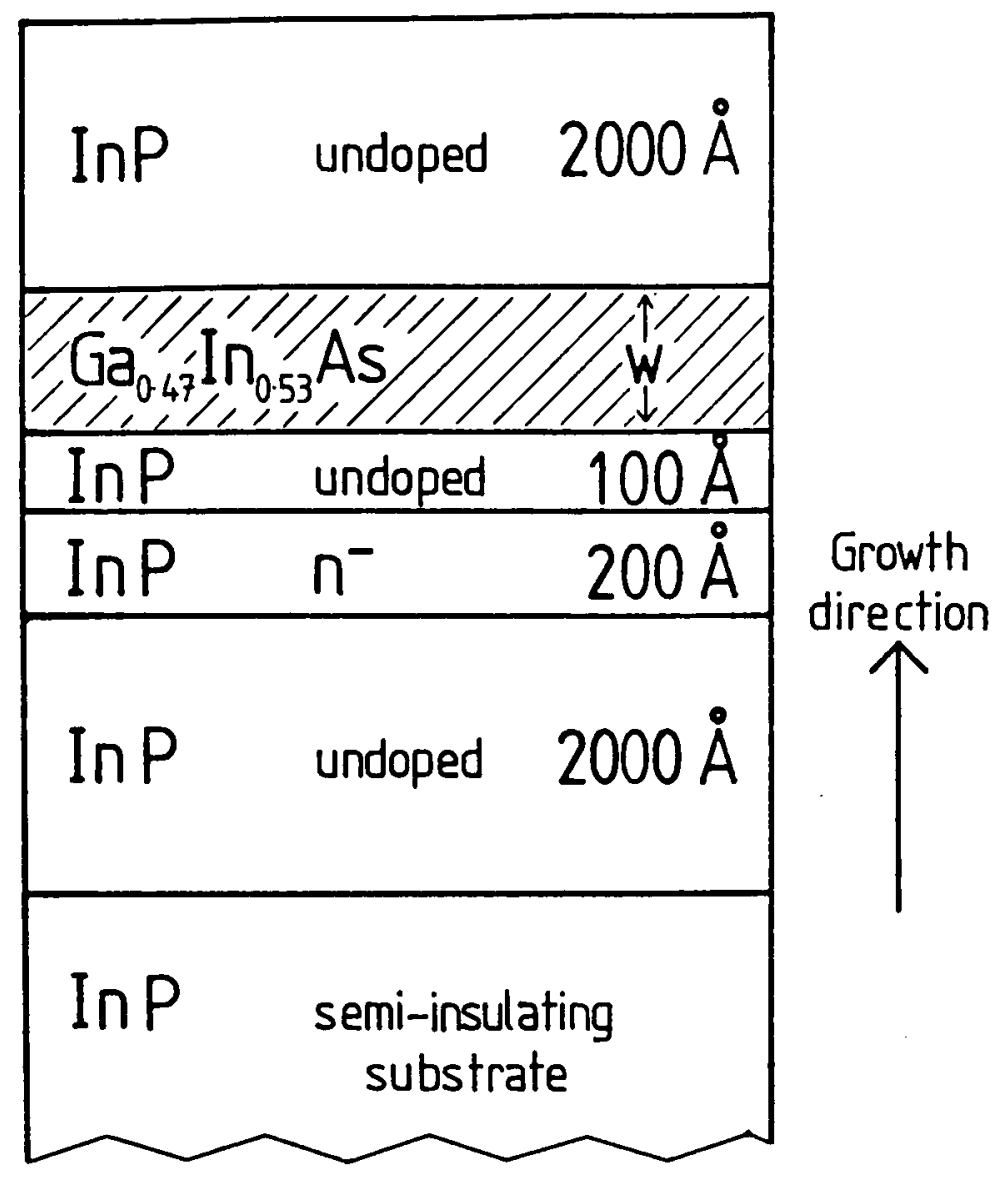

Figure 2.10 Growth profile of a single GaInAs-InP modulation doped quantum well.

modulation doping was from below the well, although R.S.R.E. have also grown structures with doped regions both sides of the quantum well. Within a given sample the carrier concentration could be changed by use of persistent photoconductivity. Well widths, carrier concentrations and mobilities are shown in Table 2.4 together with the effective masses measured by cyclotron resonance at $120 \mathrm{~K}$. The mobilies are the maximum values obtained at $77 \mathrm{~K}$.

\begin{tabular}{|c|c|c|c|c|c|c|}
\hline SAMPLE & $\begin{array}{l}\text { Doping } \\
\text { Dens it y } \\
\mathrm{cm}^{-3}\end{array}$ & $\begin{array}{l}\text { Well } \\
\text { Width } \\
\dot{A}\end{array}$ & $\begin{array}{c}\text { Carrie } \\
(77 \mathrm{~K}) \\
\text { Dark }\end{array}$ & $\begin{array}{r}\text { Density } \\
10^{1} \mathrm{~cm}^{-2} \\
\quad \text { Light }\end{array}$ & $\stackrel{\mu}{\mu} \mathrm{v}^{-1} \mathrm{~s}^{-1} \mathrm{~m}^{-2}$ & $\begin{array}{r}\mathrm{m}^{\mathrm{*}} \mathrm{CR} \\
\times 10^{4} \mathrm{~m}_{\mathrm{e}} \\
(120 \mathrm{~K})\end{array}$ \\
\hline 5599 & u.d. & 100 & 0.85 & 2.6 & 2.4 & 560 \\
\hline 5371 & u.d. & 100 & $<0.3$ & 2.0 & 2.8 & 530 \\
\hline 5899 & $2 \times 10^{17}$ & 100 & 3.7 & 5.5 & 4.8 & 500 \\
\hline 5904 & $2 \times 10^{17}$ & 100 & 3.7 & 5.3 & 6.6 & 501 \\
\hline 5506 & $3 \times 10^{17}$ & 100 & 7.6 & 9.0 & 4.2 & 549 \\
\hline 5900 & $2 \times 10^{17}$ & 200 & 4.1 & 5.5 & 5.0 & 491 \\
\hline 5901 & $2 \times 10^{17}$ & 300 & 2.8 & 3.4 & 6.0 & 482 \\
\hline 5902 & $2 \times 10^{17}$ & 500 & 5.9 & 6.8 & 7.4 & 480 \\
\hline 5903 & $2 \times 10^{17}$ & 800 & 12 & - & 6.1 & 478 \\
\hline 6014 & u.d. & 800 & 0.58 & 1.2 & - & 480 \\
\hline 5868 & $1 \times 10^{17}$ & 100 & 2.9 & 3.3 & 6.9 & 496 \\
\hline 5869 & $1 \times 10^{17}$ & 200 & 1.9 & 3.0 & 6.8 & 490 \\
\hline 5870 & $1 \times 10^{17}$ & 300 & 3.3 & 4.1 & 6.8 & 470 \\
\hline 5871 & $1 \times 10^{17}$ & 500 & 4 & - & 6.0 & 475 \\
\hline 5872 & $1 \times 10^{17}$ & 800 & 3.1 & 3.4 & - & 478 \\
\hline
\end{tabular}

Table 2.4

Sample parameters of GaInAs-InP quantum wells. 


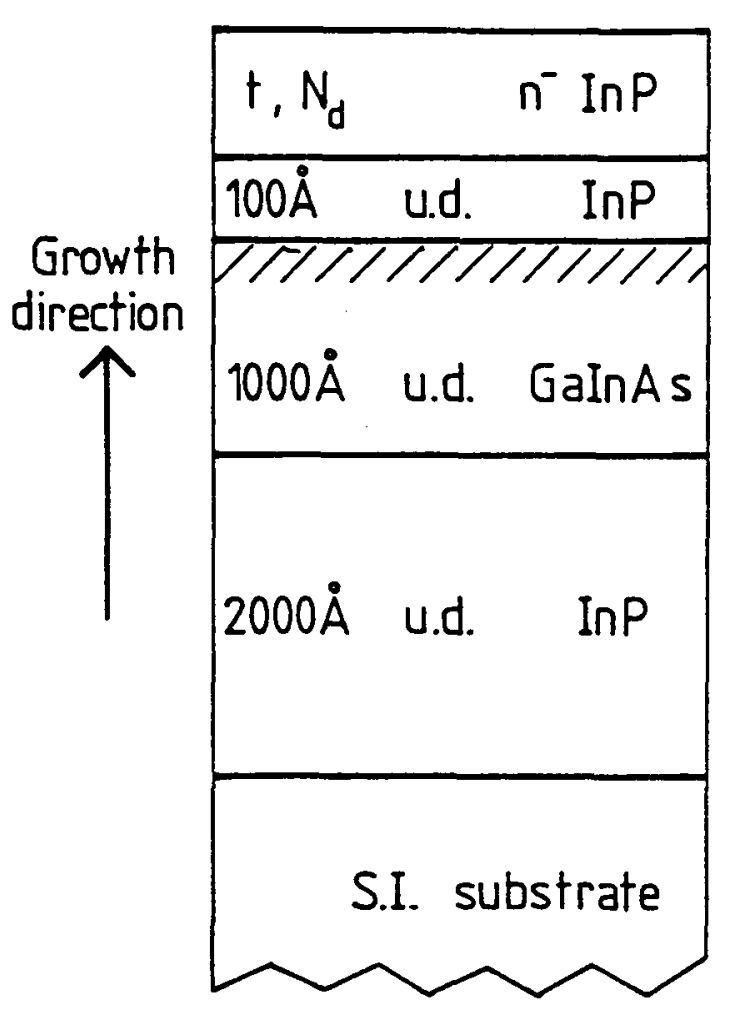

(a) Normal

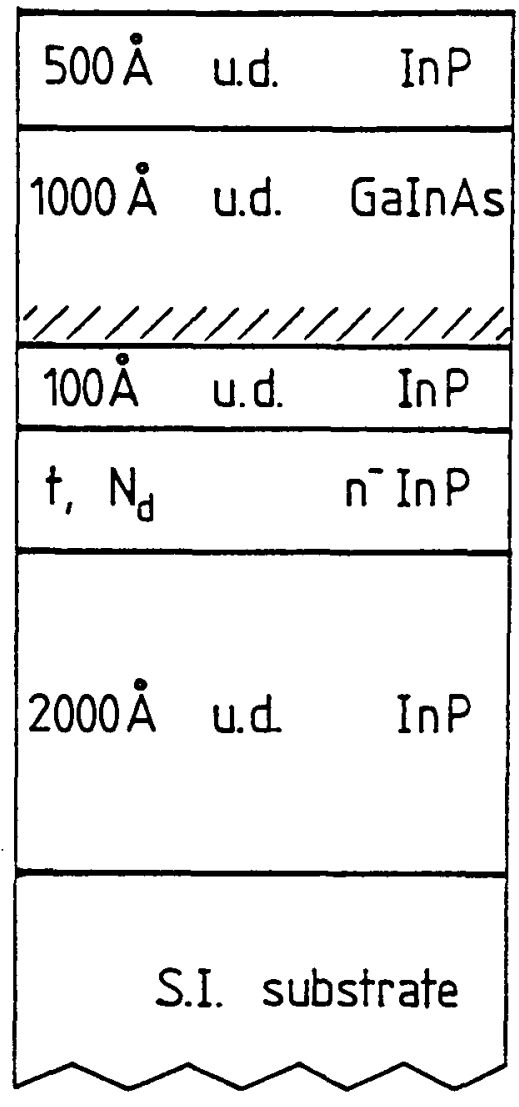

(b) Inverted

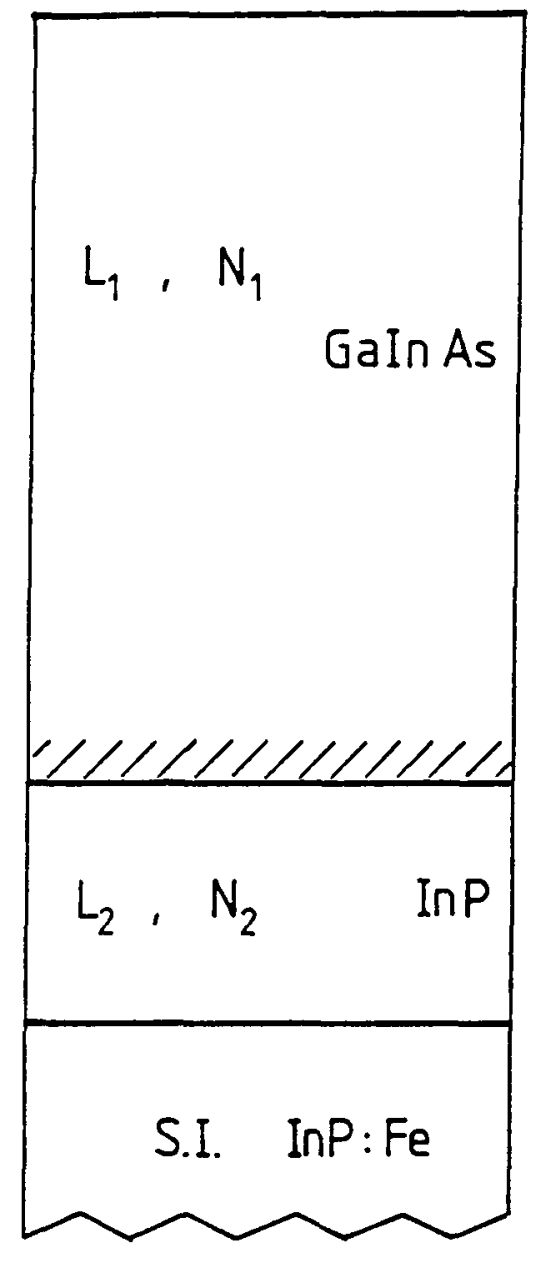

(c)

Figure 2.11 Growth profile of GaInAs-InP heterojunctions grown by R.S.R.E. with (a) normal and (b) inverted interfaces and (c) grown by Thompson-CSF.

\subsubsection{GaInAs-InP Heterojunctions}

The GaInAs-InP heterojunctions used came from two sources: those supplied by R.S.R.E. were grown by AP-MOCVD (Kane et al. 1986); and those from ThompsonCSF, were grown by LP-MOCVD (Razeghi et al. 1982). Schematic diagrams of each type of heterojunction are displayed in Fig. 2.11, which shows the major difference in the two groups of structures. The R.S.R.E. samples are more complex, being modulation doped and a mixture of samples with "normal" and "inverted" interfaces. Kane et al. (1985) found that, in contrast to GaAs-GaAlAs, the transport properties of

\begin{tabular}{|c|c|c|c|}
\hline SAMPLE & $\begin{array}{c}\text { Int erface } \\
\text { type }\end{array}$ & $\begin{array}{c}\mathrm{n}_{\mathrm{s}} \\
10^{1} \mathrm{~cm}^{-2}\end{array}$ & $\begin{array}{c}\mathrm{m}^{*} \mathrm{CR} \\
\times 10^{4} \mathrm{~m}_{\mathrm{e}}\end{array}$ \\
\hline 5237 & normal & $6-7$ & 512 \\
5248 & inverted & 4.9 & 505 \\
5228 & normal & $4.1-7.6$ & 476 \\
\hline
\end{tabular}

Table 2.5

Sample parameters of R.S.R.E. grown GaInAs-InP heterojunctions. 
a 2 DEG at inverted or normal interfaces were very similar. The $800 \AA$ wide quantum wells of the previous section $(6014,5903$ \& 5872) also behaved effectively like heterojunctions. Table 2.5 gives the carrier concentrations and cyclotron resonance effective masses (at 120K) for each sample.

The thicknesses and doping densities $\left(\mathrm{N}_{\mathrm{i}}=\mathrm{N}_{\mathrm{d}}-\mathrm{N}_{\mathrm{a}}\right)$ of the layers shown in Fig. 2.12c for the french heterojunctions are shown in Table 2.6. Shubnikov-de Haas measurements showed that higher subbands were occupied in a number of samples (Razeghi et al. 1986). Table 2.6 also gives the carrier concentrations, mobilities and numbers of subbands occupied at $4 \mathrm{~K}$ and cyclotron resonance effective masses at $120 \mathrm{~K}$ for each sample.

\begin{tabular}{|l|c|c|c|c|c|c|c|c|}
\hline SAMPLE & $\begin{array}{c}\mathrm{L}_{1} \\
\mu \mathrm{m}\end{array}$ & $\begin{array}{c}\mathrm{N}_{1} \\
\mathrm{Cm}^{-3}\end{array}$ & $\begin{array}{c}\mathrm{L}_{2} \\
\mathrm{~nm}\end{array}$ & $\begin{array}{c}\mathrm{N}_{2} \\
\mathrm{~cm}^{-3}\end{array}$ & $\begin{array}{c}\mu_{4 \mathrm{~K}} \\
\mathrm{~V}^{-1} \mathrm{~s}^{-1} \mathrm{~m}^{2}\end{array}$ & $\begin{array}{c}\mathrm{n}_{\mathrm{s}} \\
10^{1} \mathrm{~cm}^{-2}\end{array}$ & $\begin{array}{c}\mathrm{N} \text { o of } \\
\text { subbands }\end{array}$ & $\begin{array}{c}\mathrm{m}^{*} \mathrm{CR} \\
\times 10^{4} \mathrm{~m}_{\mathrm{e}}\end{array}$ \\
\hline 83 & 2.0 & $10^{15}$ & 100 & $10^{14}$ & 2.2 & $1.3-2.2$ & 1 & 486 \\
$82 \mathrm{~A}$ & 0.4 & $10^{15}$ & 50 & $10^{14}$ & 4.3 & 3.2 & 2 & 490 \\
$82 \mathrm{~B}$ & 0.4 & $10^{15}$ & 50 & $10^{14}$ & 3.4 & 4.6 & 2 & 490 \\
90 & - & - & - & - & 4.2 & 4.9 & 2 & - \\
92 & 1.0 & $10^{15}$ & 150 & $10^{16}$ & 5.4 & 5.2 & 3 & 478 \\
93 & 1.0 & $10^{15}$ & 150 & $10^{16}$ & 4.1 & 5.5 & 3 & 478 \\
$52 \mathrm{~A}$ & 1.2 & $10^{15}$ & 100 & $10^{14}$ & 4.4 & 5.6 & 3 & 488 \\
$52 \mathrm{C}$ & 1.2 & $10^{15}$ & 100 & $10^{14}$ & 3.8 & 6.0 & 3 & 488 \\
53 & 1.2 & $10^{15}$ & 100 & $10^{14}$ & 3.1 & 5.8 & 3 & 487 \\
\hline
\end{tabular}

\section{Table 2.5}

Sample parameters of Thompson-CSF GaInAs-InP heterojunctions.

The Thompson heterojunctions had Hall bars defined by photolithography which were somewhat larger than the Philips bars. In the notation of Table 2.1 the length between voltage contacts was $l \sim 3 \mathrm{~mm}$ and the bar width $\mathrm{w} \sim 300 \mu \mathrm{m}$, giving aspect ratios $\sim 10$.

Samples from R.S.R.E. were supplied as part wafers and so had to be sawn into narrow bars. As it is difficult to form reliable ohmic contacts right at the edge of the bar by diffusing indium dots, values of $n_{s}$ deduced from the Hall voltage had to be calibrated by checking with Shubnikov-deHaas oscillations at $4.2 \mathrm{~K}$ and assuming that the fraction of current flowing between Hall contacts was constant with temperature. 
CHAPTER 3

MAGNETOPHONON RESONANCE IN TWO-DIMENSIONAL

GaAs-GaAlAs STRUCTURES

3.1 Introduction

3.2 Damping of the Oscillations

3.2.1 Experimental Considerations 64

3.2.2 Quantiative Results 66

$\begin{array}{ll}\text { 3.2.3 Discussion } & 68\end{array}$

3.3 Magnetophonon Resonance Amplitudes

3.3.1 Experimental Considerations $\quad 70$

$\begin{array}{ll}\text { 3.3.2 Temperature Dependence } & 75\end{array}$

$\begin{array}{ll}\text { 3.3.3 Carrier Concentration Dependence } & 78\end{array}$

3.3.4 Discussion of Resonance Amplitudes 83

3.4 Magnetophonon Effective Mass

3.4.1 Non-parabolicity $\quad 88$

3.4.2 Polaron Coupling $\quad 89$

$\begin{array}{ll}\text { 3.4.3 Calculation of Magnetophonon Mass } & 90\end{array}$

$\begin{array}{ll}\text { 3.4.4 Upper Subband Contributions } & 91\end{array}$

3.5 Resonance Positions and Phonon Frequencies

$\begin{array}{lll}3.5 .1 & \text { Peak Shifts } & 93\end{array}$

$\begin{array}{ll}\text { 3.5.2 The Measured Values } & 94\end{array}$

$\begin{array}{ll}\text { 3.5.3 Interpretation of the Positions } & 98\end{array}$

3.6 Magnetophonon Oscillations in the Hall Coefficient 102

3.7 Rotation in Magnetic Field

3.7.1 Effect on Resonance Amplitudes 103

3.7.2 Effect on Resonance Position 106

$\begin{array}{lll}3.8 & \text { Resonances in GaAs-GaAlAs Quantum Wells } & 107\end{array}$

3.9 Discussion of Possible Explanations

3.9.1 Phonon Modes $\quad 111$

3.9.4 Interface Phonons 112

$\begin{array}{ll}3.9 .3 \text { Subband Structures } & 114\end{array}$

3.9.4 Coupling to TO Phonons? 115

$\begin{array}{ll}\text { 3.9.5 Coupled Plasmon-Phonon Modes } & 116\end{array}$

$\begin{array}{lll}3.10 & \text { Summary } & 118\end{array}$ 


\section{$\underline{3.1 \quad \text { INTRODUCTION }}$}

The magnetophonon effect was first predicted by Gurevich \& Firsov in 1961 and the first experimental observations were made by Puri \& Geballe (1963) in InSb. It arises from the resonant absorption of optic phonons, causing electrons to make transitions between the quantized energy states produced in a magnetic field. This results in a resonant relaxation of the electron momentum and hence oscillatory contributions to the resistivity and other transport coefficients. Conservation of crystal momentum limits the wavevectors of phonons involved to a region very close to the centre of the Brillouin Zone where the optic phonons are essentially monoenergetic, giving a sharp resonance condition:

$$
\hbar \omega_{0}=\mathrm{N} \hbar \omega_{\mathrm{c}}=\mathrm{N} \hbar \mathrm{eB} \mathrm{N} / \mathrm{m}^{*}, \quad \mathrm{~N}=1,2,3, \ldots
$$

where $\mathrm{m}^{*}$ is a suitably defined effective mass discussed below, the product $\mathrm{NB}_{\mathrm{N}}=\mathrm{B}_{\mathrm{F}}$ is the fundemental resonance field and $\omega_{0}$ is the optic phonon frequency. In high magnetic fields the conductivity $\sigma_{\mathbf{x x}}$ is proportional to the scattering rate and hence to the joint density of states for transitions between two levels. The conductivity thus exhibits a maximum whenever the above resonance condition is satisfied, and since $\rho_{x x} \propto \sigma_{x x}$ in high fields, there will aslo be maxima in resistivity, which is the experimentally measured quantity.

\section{$\underline{\text { Review }}$}

Magnetophonon resonance has been extensively studied in bulk semiconductors and has been reviewed several times eg. Harper, Hodby \& Stradling 1973, Peterson 1975 and Nicholas 1985. Most studies have concentrated on using the resonance positions to deduce either the electron effective mass or the frequencies of optic phonon modes interacting with the electrons. The dominant interaction is usually with longitudinal optic phonons because their bulk polarisation allows coupling to electrons, whereas transverse modes with only a surface polarisation do not show strong coupling. However there have been some reports of interactions with other modes in the hot electron region (Stradling \& Wood 1970b), eg. pairs of zone edge acoustic phonons (Eaves et al. 1974); pairs of LO phonons (Stradling \& Wood 1968). The effective masses are 
always found to be greater than the band edge value, due to non-parabolicity of the conduction band and enhancement from polaron coupling. In ternary and quaternary alloy materials magnetophonon series have been found to arrise from the optic phonon modes of each constituent binary (Nicholas et al. 1979).

In $\mathrm{Si}$ and $\mathrm{Ge}$, the shape of the conduction band is complicated by spin and valley degeneracies and this produces series from both inter- and intra-valley transitions (Eaves et al. 1975). It is also possible to have transitions between impurity states of well defined energy and the Landau levels of the conduction band, giving rise to the magneto-impurity effect which was reviewed by Eaves \& Portal (1979).

Series of oscillations are not only observed in the transverse magnetoresistance, $B \perp j$. In the longitudinal configuration, with $B$ parallel to $j$, rather complicated series arrise due to a number of possible resonance and "pseudo-resonance" mechanisms, some of which may involve two and even three phonon processes. Oscillations have also been seen in the Hall coefficient (Wood et al. 1970) which may occur as series of maxima or minima depending on the material.

There has also been considerable study of the oscillation amplitudes, both experimental (Eaves et al. 1975, Fujisawa 1978, Senda et al. 1979) and theoretical (Barker 1970, 1972). Of particular interest has been the way in which the resonances form an exponentially damped series with a direct relation between the experimental damping factor and broadening of the Landau levels.

In two dimensional systems there has now been a decade of study and, as will shortly be demonstrated, many fundemental questions remain unanswered and new discoveries continue to be made. The first report was made by Tsui et al. (1980) on GaAs-GaAlAs heterostructures and extended to higher magnetic fields by Englert et al. (1982b) and Kido et al. (1982). All these assumed the dominant interaction to be with bulk GaAs LO phonons, and thus deduced values for the effective mass. By contrast Brummell et al. (1987 \& 88) used effective mass values measured by cyclotron resonance and deduced phonon frequencies substantially lower than the bulk LO value, and speculated about coupling to different phonon modes. Grégoris et al. (1988) 
investigated the effects of hydrostatic pressure on the resonances, finding that both the mass and resonance amplitudes increase with pressure. Mori et al. (1988a) used a single quantum well and found series of resonances from inter-subband transitions as well as the usual intra-subband series.

The other $2 \mathrm{D}$ systems to have received significant attention have electrons localised in the ternary alloy GaInAs, lattice matched to barriers of either InP or AlInAs. These show several interesting phenomena due to the two mode behaviour of the GaInAs as well as interactions with the phonons of the barrier materials (Portal et al. 1983, 1984 and Brummell et al. 1983).

\section{Overview of This Study}

In the last chapter the theory of transverse magnetophonon resonance in two-dimensional systems was reviewed. The next three chapters will be concerned with experimental measurements and are divided as follows:

Chapter 3 deals with measurements made on a range of high mobility GaAs-GaAlAs heterojunctions and (to a limited extent) quantum wells. A systematic study of the resonance amplitude as a function of carrier concentration has been made, from which conclusions may be drawn regarding the changes in strength of electron-phonon coupling and Landau level broadening. The descrepancies in phonon frequencies/effective mass values, alluded to above, are also discussed in relation to differences in behaviour between heterojunctions and quantum wells. In addition the influence of tilting the magnetic field direction is studied, which has the surprising result of drastically reducing the amplitude of the oscillations, and gives an anomalous angular dependence of the field positions.

In Chapter 4 GaInAs-InP heterostructures are investigated. These show more marked differences between quantum wells and heterojunctions than in GaAs especially on rotation. Suprisingly, the phonon frequencies deduced display a dramatic reduction as the electon concentration is increased. Reasons for this behaviour are investigated which call into question the phonon structure of GaInAs.

The third chapter on the magnetophonon effect (Chapter 5) is dedicated to warm 
electron effects where phonon emision processes become important, leading to reversals in the sign of the magnetophonon extrema. Competition between the absorption and emision effects are studied through the temperature and electric field dependences of the resonance amplitudes and field positions. Theoretical implications of the various possible energy loss mechanisms are then considered and related to the experimental results.

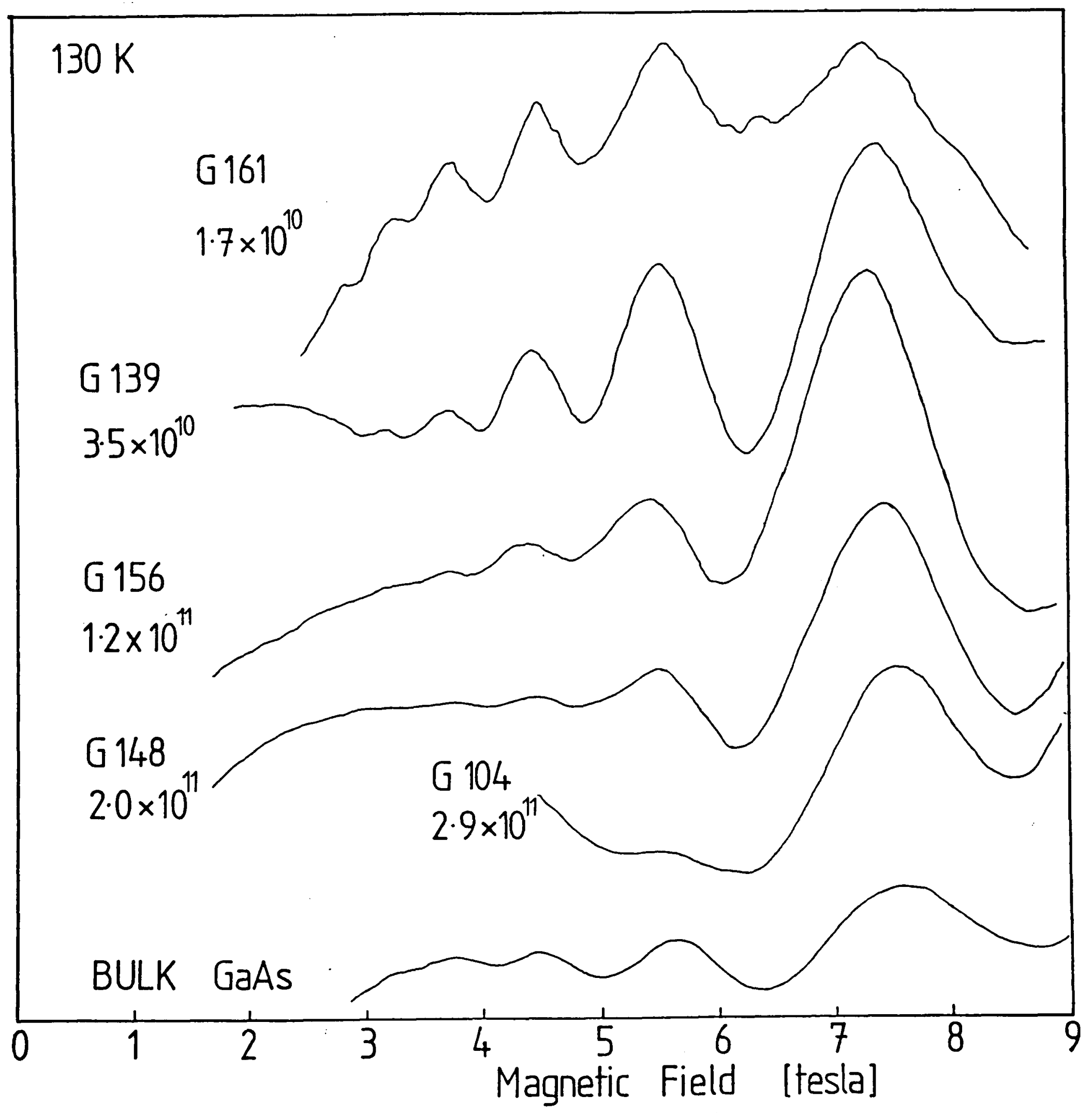

Figure 3.1 Experimental recordings of magnetophonon resonance in GaAs-GaAlAs heterojunctions, with a linear term in $B$ subtracted, at various carrier concentrations, indicated on the figure in $\mathrm{cm}^{-2}$. The last trace is from bulk GaAs for comparison. 


\subsection{DAMPING OF THE OSCILLATIONS}

\subsubsection{Experimental Considerations}

The damping factor was shown in Section 2.3 to be related to the broadening of the Landau levels and hence the dominant scattering processes, which makes its study extremely important and allows more direct insight into the underlying behaviour than revealed by the absolute magnitude of the oscillations.

Typical experimental results using the compensation technique described in Section 2.7.2 are shown in Fig. 3.1 for GaAs-GaAlAs heterojunctions with electron concentrations between 0.17 and $2.9 \times 10^{11} \mathrm{~cm}^{-2}$ and for bulk GaAs. The damped series of oscillations discussed in Section 2.3 is seen, with the damping being stronger at higher carrier concentrations and generally more than for the 3-D case.

Quantitiative results for the damping factor, $\tilde{\gamma}$, of the empirical equation 2.18 will shortly be presented, but first a few notes on the measurement of this quantity. On a direct (or compensated) trace the damping factor may be measured between adjacent peaks, with $\mathrm{N}=\mathrm{i}$ and $\mathrm{N}=\mathrm{j}(\mathrm{j}=\mathrm{i}+1)$ as

$$
\tilde{\gamma}_{\mathrm{ij}} \quad=\ln \left[\frac{\Delta \rho_{\mathrm{i}}}{\Delta \rho_{\mathrm{j}}}\right]
$$

In some cases the recordings. were of the second derivative of the magnetoresistance, as described in Section 2.7.2. This creates some uncertainty in the measurement of $\tilde{\gamma}$ since the amplitude $\left(A_{N}\right)$ of each peak will depend on the response function, $f(\omega)$, of the filters. Writing $A_{N}=\Delta \rho f(\omega)$, where $f(\omega)$ will depend only on the sweep rate and the resonance field, the damping factor for a series of oscillations of fixed periodicity in 1/B, becomes:

$$
\tilde{\gamma}_{i j}=\ln \left[\frac{A_{i}}{A_{j}}\right]+\ln \left[f_{j} / f_{i}\right]
$$

with the final term being a constant for each pair of resonances if a fixed sweep rate is used. This allows $\tilde{\gamma}$ to be compared between traces from different samples or at different temperatures and an absolute value to be found by comparison with a suitable compensated trace. 


\section{Peak Sharpening}

From Eqns. 2.18 and 2.22 it can be seen that the empirical damping factor is only a theoretically useful quantity when harmonics of Eqn. 2.22 can be neglected compared to the first term in the series. When this is not so terms from the higher order series will lead to peak sharpening of the resonances. This will be most pronounced at high field (low N) and for small damping factors. In bulk InSb Stradling \& Wood (1970) saw very pronounced peak sharpening. In these 2-D GaAs samples $\tilde{\gamma}$ changes with $\mathrm{n}_{\mathrm{s}}$, as shown in Fig. 3.2, which suggest peak sharpening may be evident at low, but not at high, carrier densities. On close inspection of Fig. 3.1 some evidence may be found for peak sharpening at the lowest carrier concentration, supporting this general idea, but the series are mostly harmonic, meaning that it is reasonable to make deductions from the measured values of $\tilde{\gamma}$. [Particular note should be made that these are all compensated traces. In differentiated traces there is apparantly more sharpening of the peaks, but this is due to the frequency response of the filters.]

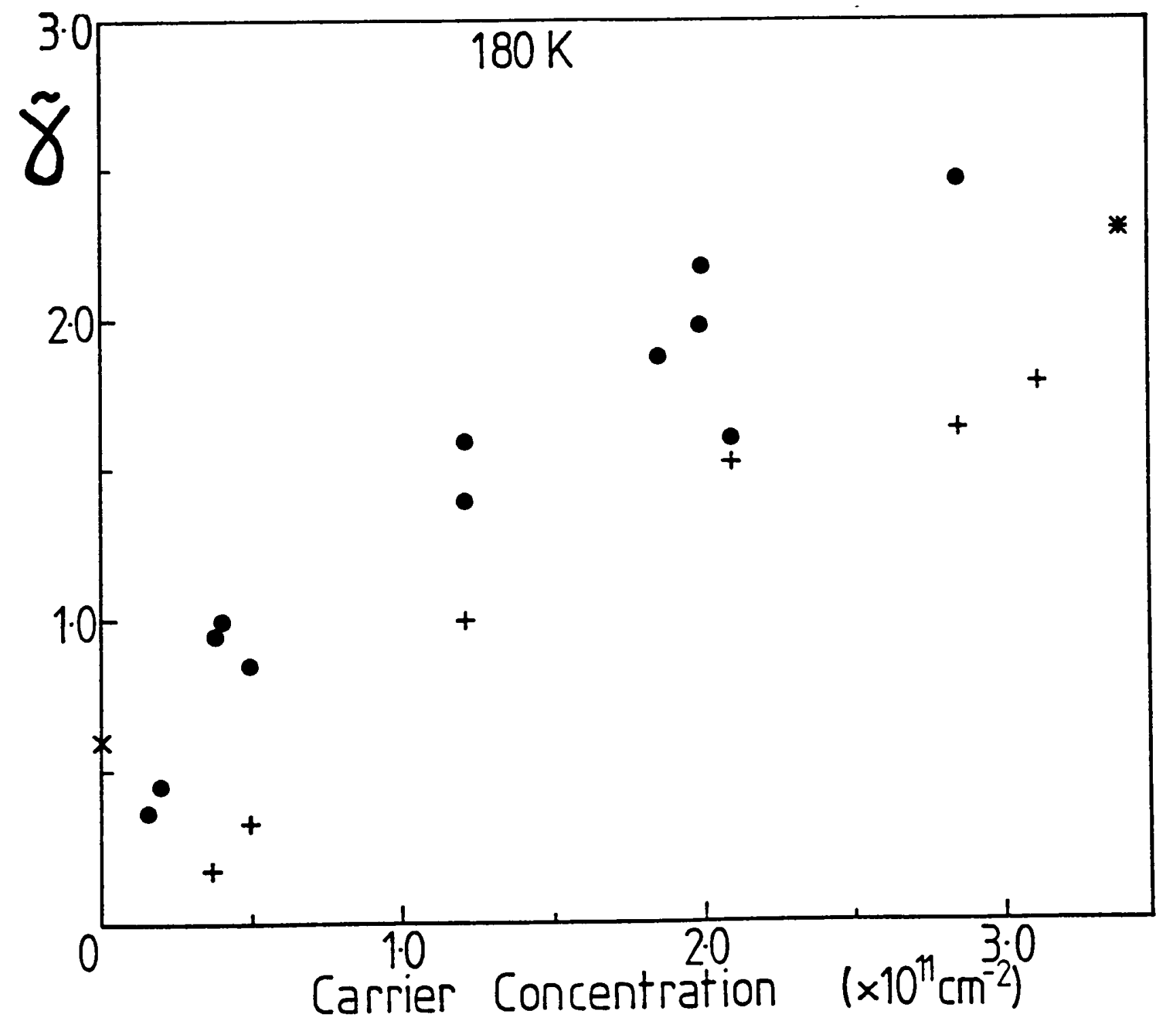

Figure 3.2 The damping factor, $\tilde{\gamma}$, as measured between the $N=2 \& 3(+)$ and the $\mathrm{N}=3 \& 4(0)$ peaks at $180 \mathrm{~K}$. The $\times$ corresponds to the $3 \mathrm{D}$ value and the * is from the data of Englert et al. (1982). 


\subsubsection{Quantitative Results}

\section{Variation of $\gamma$ with Carrier Concentration}

The variation of $\tilde{\gamma}$ with carrier density, measured from the $N=3$ and 4 resonances on compensated traces at $180 \mathrm{~K}$, is shown in Fig. 3.2 , including a value from the data of Englert et al. 1982b. In modulation doped heterojunctions the carrier concentration may be increased by reducing the spacer layer thickness, $L_{s}$, which suggests there should be a correspondance between damping and spacer layer thickness. This can be seen in Fig. 3.3. As well as increasing $n_{s}$, reducing $L_{s}$ increases the strength of remote ionised impurity scattering by bringing the ionised donors closer to the 2DEG. That $\tilde{\gamma}$ shows such a convincing correlation with $\mathrm{L}_{\mathrm{s}}$ suggests that the damping factor is heavily influenced by remote ionised impurity scattering. The star in Fig. 3.3 is from the data of Kido et al. (1982) who used similar structures; and the odd point below the general trend is from G215. [The n-type buffer layer means the sample is quasi-accumulation layer like with additional electrons in the 2DEG which screen the impurity potential and thus narrow the levels.]

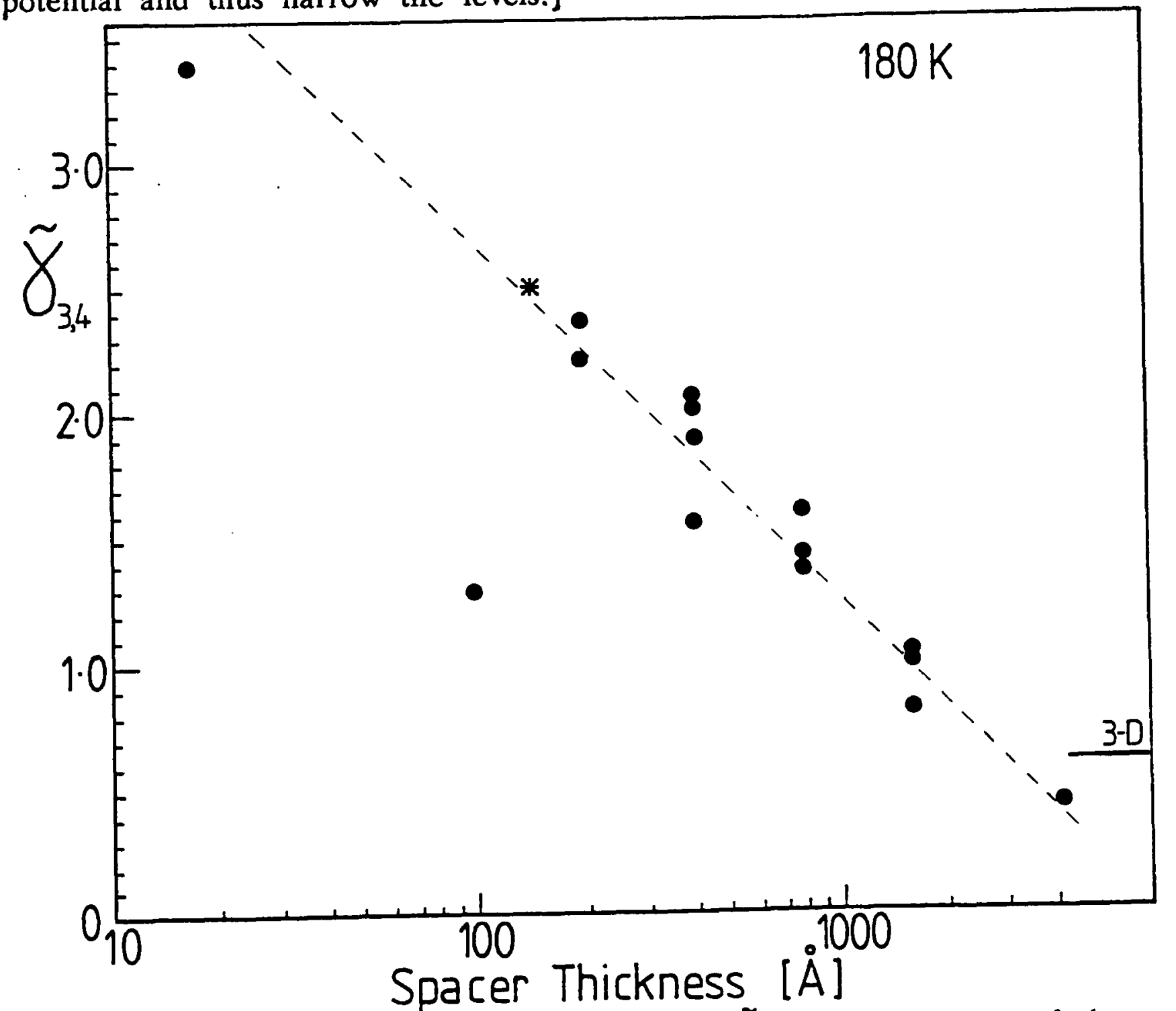

Figure 3.3 Variation of the empirical damping factor, $\tilde{\gamma}_{34}$, with thickness of the spacer layer in modulation doped GaAs-GaAlAs heterojunctions at $180 \mathrm{~K}$. 


\section{Variation of $\gamma$ with Temperature}

The damping factor is also found to vary with temperature as illustrated in Fig. 3.4 for G139, G29 and $\mathrm{G} 104$ with $\mathrm{n}_{\mathrm{s}}=0.5,2.0$ and $2.8 \times 10^{11 \mathrm{~cm}-2}$ respectively. In this case the variation is linear in $T$ at high temperature, however at lower temperatures there is more scatter in the data and the trend is for a slower variation. This is in qualitative agreement with measurements on bulk GaAs (Eaves et al. 1975, Senda et al. 1979, Kido \& Miura 1983) where the observed behaviour was $\tilde{\gamma} \propto \mathrm{T}$ above $\sim 130 \mathrm{~K}$ and $\tilde{\gamma} \propto \mathrm{T}^{\frac{1}{4}}$ at lower temperatures where multiple impurity scattering (band-tailing) is the dominant interaction.

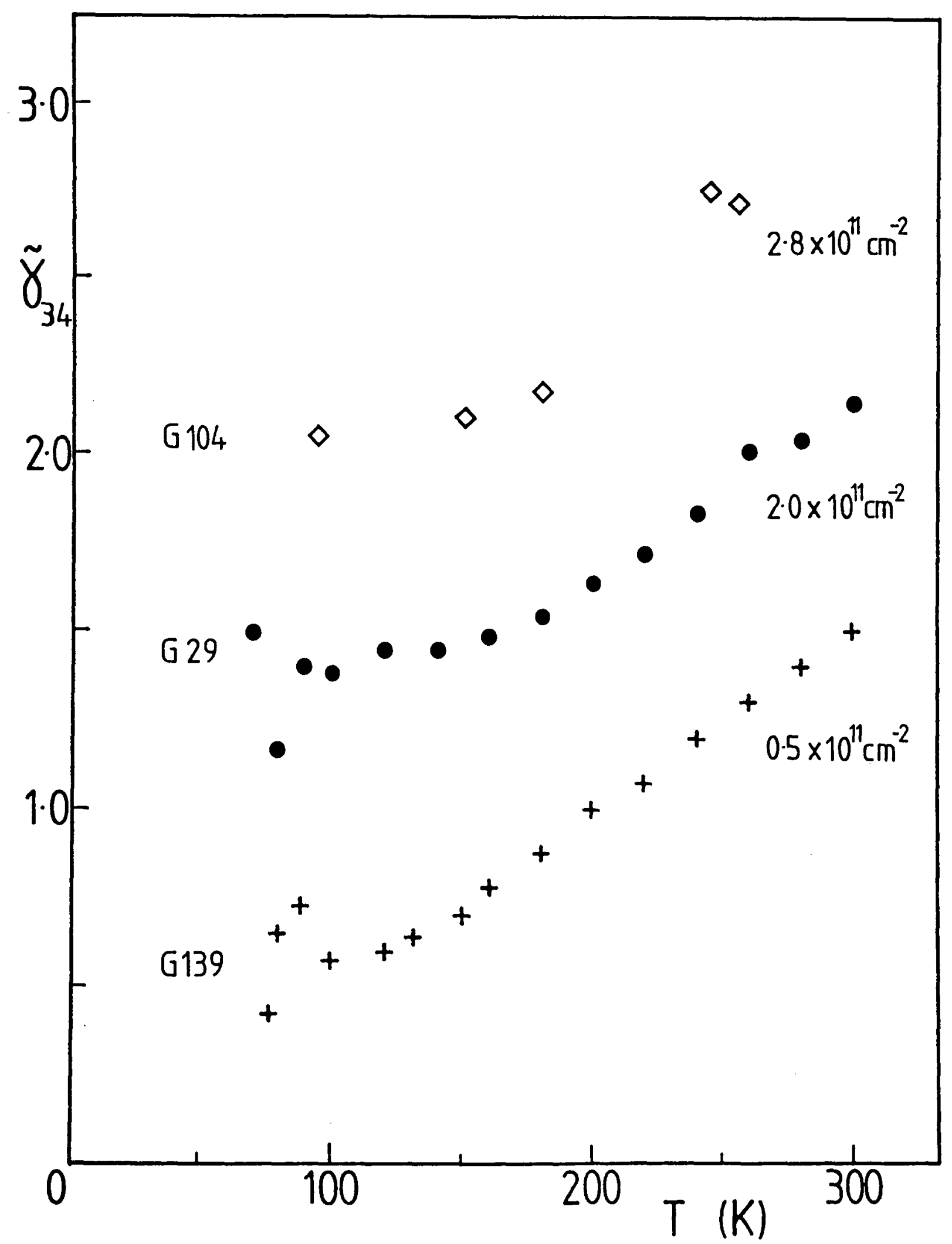

Figure 3.4 Temperature variation of $\tilde{\gamma}_{34}$ for three GaAs-GaAlAs heterojunctions with the carrier concentrations indicated on the left. 


\section{Variation of $\tilde{\gamma}$ with Magnetic Field}

$\tilde{\gamma}$ is not constant between all pairs of resonances in a given trace. This is partly due to additional unresolved peaks coming from holes in the unintentionally p-type GaAs (see Section 3.7), but usually it appears to decrease towards higher fields with the approximate result that $\tilde{\gamma}_{45}-\tilde{\gamma}_{34} \sim 0.5$ and $\tilde{\gamma}_{34}-\tilde{\gamma}_{23} \sim 0.4$

\section{$\underline{3.2 .3 \quad \text { Discussion }}$}

From Figs. 3.3 and 3.4 the damping factor in these modulation doped heterojunctions can be empirically described by:

$$
\tilde{\gamma}=2.8+0.5 \frac{\omega_{0}}{\omega_{c}}+\frac{T}{200}-0.6 \operatorname{lnL} s
$$

with $\mathrm{L}_{\mathrm{s}}$ in angstrom. The constant term probably depends on the background impuity level in the GaAs. (Experiments to test this hypothesis by using samples with impurities introduced deliberately in the region of the 2DEG are being contemplated.)

Further evidence that $\tilde{\gamma}$ depends on ionised impurity scattering comes from the magnetic field dependence, where we observe a small decrease with field. In calculations for bulk semiconductors Barker (1972) found that for phonon scattering (both acoustic and optic) $\gamma \propto \mathrm{B}^{2 / 3}$ and for impurity scattering $\gamma$ is independent of magnetic field, but increases with the square root of the impurity density. In $2 \mathrm{D}$

Mori et al. (1988b) again found $\gamma$ to be field independent for impurity scattering but to vary like $\sqrt{ } \mathrm{B}$ for phonon scattering. Thus our results are closer to behaviour predicted for impurity scattering than the $B^{\frac{1}{2}}$ behaviour for phonon limited processes.

Equation 3.4 is only valid in the high temperature limit where $\tilde{\gamma} \propto \mathrm{T}$, whilst at lower temperatures we observe a slower variation. This can be explained by considering the various scattering mechanisms responsible for the level broadening.

Mori et al. (1988b) evaluated $\Gamma$ for several forms of scattering in a similar structure to our samples (but with somewhat different parameters). Their result for the temperature dependence of $\Gamma$ due to remote impurity $\left(\Gamma_{\text {RIS }}\right)$ and optic phonon scattering $\left(\Gamma_{\text {OPS }}\right)$ is reproduced in Fig. 3.5, where it can be seen that whilst remote impurity scattering dominates, the contribution from phonon scattering will become important at 

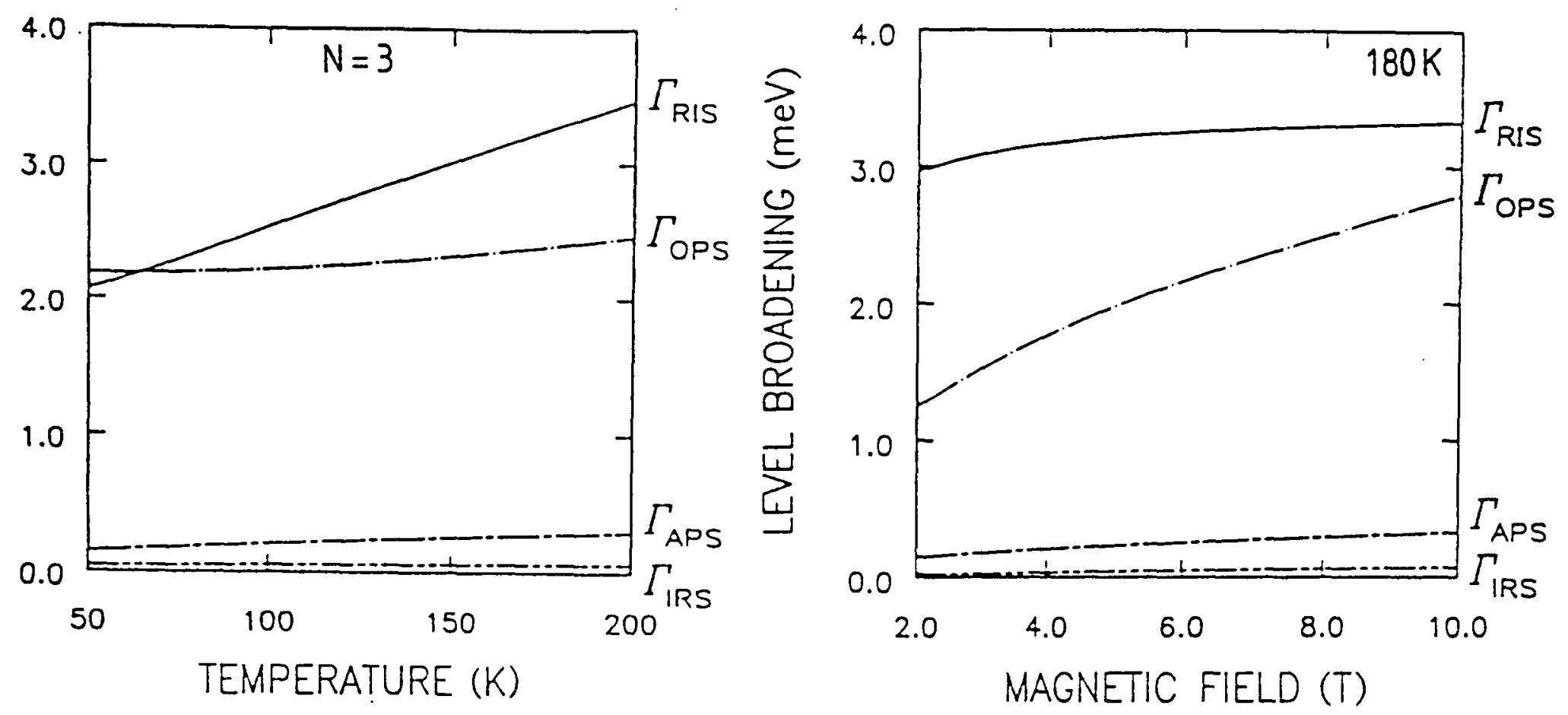

Figure 3.5 Calculations of temperature and magnetic field dependence of the Landau level broadening due to scattering by remote impurities, $\Gamma_{\mathrm{RIS}} ;$ optical phonons, $\Gamma_{\mathrm{OPS}} ;$ acoustic phonons, $\Gamma_{\mathrm{APS}} ;$ and interface roughness, $\Gamma_{\mathrm{IRS}}$ Background impurity scattering appears an order of magnitude below $\Gamma_{\text {IRS }}$. Parameters used: $\mathrm{n}_{\mathrm{s}}=3 \times 10^{11} \mathrm{~cm}^{-2} ; \mathrm{L}_{\mathrm{s}}=100 \AA ; \mathrm{N}_{\mathrm{dep}}=5 \times 10^{10} \mathrm{~cm}^{-2}$.

lower temperatures. The origin of the temperature variation of $\Gamma_{\text {RIS }}$ comes from the change in effective screening length as the electron kinetic energy increases and the system becomes less degenerate at higher temperatures, whilst $\Gamma_{\text {OPS }}$ is determined by the phonon occupation number.

The total level width, $\Gamma$, will depend on the scattering rates due to each process, $S_{i}$, and the density of final states into which scattering may occur. The number of states per unit area in each Landau level is $e B / h$, so for a level of width $\Gamma$, the density of final states is $\mathrm{eB} / \mathrm{h} \Gamma$ giving

$$
\begin{aligned}
& \Gamma \propto \sum \mathrm{S}_{\mathrm{i}} \frac{\mathrm{eB}}{\mathrm{h} \Gamma} \\
& \therefore \quad \Gamma^{2} \propto \Sigma \mathrm{S}_{\mathrm{i}}
\end{aligned}
$$

If there were only one scattering mechanism we would find that $S_{i} \propto \Gamma_{i}^{2}$, where $\Gamma_{i}$ is the width that would be calculated for that process, and hence:

$$
\Gamma^{2} \sim \Sigma \Gamma_{i}^{2}
$$

Thus it can be seen that the level widths arising from each scattering process are not 
simply additive. A similar argument applies to the damping factor and shows that the effects of different scattering mechanisms will have to be properly convolved to give the correct behaviour for $\gamma$.

At $180 \mathrm{~K}$ we have shown that $\tilde{\gamma}$ is dominated by remote impurity scattering, so the linear variation with $\mathrm{T}$ shown in Fig. 3.4 is in general agreement with the calculation of $\Gamma_{\text {RIS }}$. Any contribution from $\Gamma_{\text {OPS }}$ should be the same for all samples and would be expected to be more evident at lower carrier densities where $\Gamma_{\text {RIS }}$ is smaller. However, Fig. 3.4 shows no significant deviations at low $n_{s}$, in fact $\tilde{\gamma}$ appears linear in $T$ to lower temperatures in G139, and so it must be concluded that optic phonon scattering has a negligible effect on the level widths compared to impurity scattering.

\subsection{MAGNETOPHONON RESONANCE AMPLITUDES}

Magnetophonon resonances were observed between $60 \mathrm{~K}$ and $300 \mathrm{~K}$ as illustrated in Fig. 3.6 for sample $\mathrm{G} 148$ with an electron concentration of $2 \times 10^{11} \mathrm{~cm}^{-2}$, and a systematic study of the amplitudes of these resonances as a function of temperature and carrier concentration is the subject of this next section. To establish absolute values of the amplitudes contains more pitfalls than the relative measurements of the preceeding section, so some discussion of the method used will follow.

\section{$\underline{3.3 .1 \quad \text { Experimental Considerations }}$}

All the quantitative data was taken from compensated traces and, to eliminate non-linear terms in the background magnetoresistance, a smooth curve was drawn connecting the minima and the amplitude $(\Delta \rho)$ measured as the maximum vertical height above this line.

There are at least three ways in which resonance amplitudes could be compared: (i) directly as $\Delta \rho$; (ii) relative to the zero-field resistivity $-\Delta \rho / \rho_{0}$; or (iii) relative to the resistivity at the field of the resonance $-\Delta \rho / \rho_{\mathrm{B}}$. All three have been used in previous studies and it is not altogether clear which is to be prefered, but it should be noted that each has a different physical significance. Straight comparisons of $\Delta \rho$ between samples requires the dimensions of the active area of each to be well known but avoids 


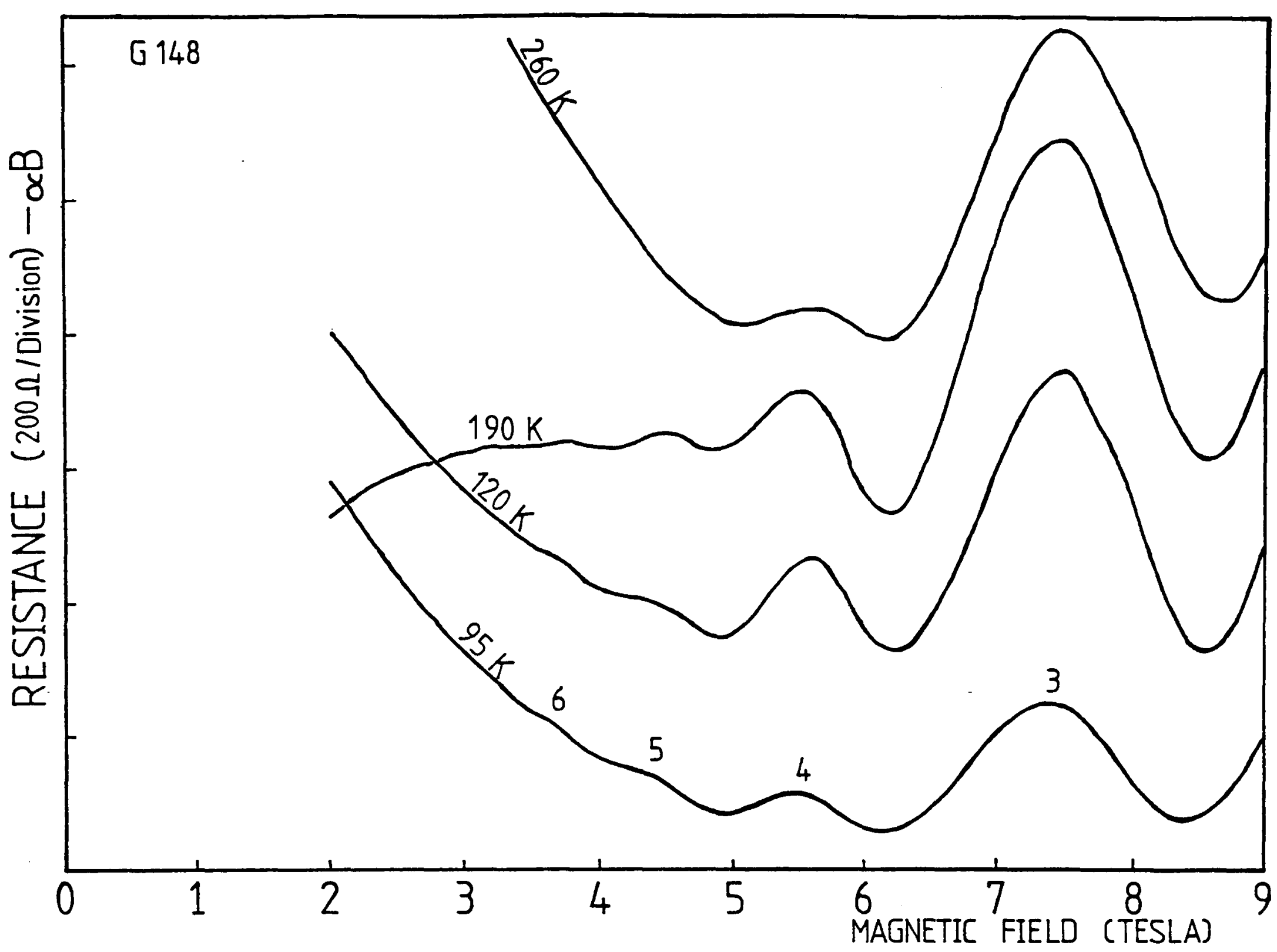

Figure 3.6 Temperature variation of magnetophonon resonance illustrated by example compensated traces between $260 \mathrm{~K}$ and $95 \mathrm{~K}$ for G148.

introducing non-resonant artifacts of the resistivity into the oscillations. In theories based on the Kubo formula $\sigma_{x x}$ is the calculated quantity and for small oscillations $\Delta \sigma_{\mathrm{xx}} / \sigma_{\mathrm{xx}}=\Delta \rho_{\mathrm{xx}} / \rho_{\mathrm{xx}}$. Thus $\Delta \rho / \rho$ is proportional to the scattering per electron, but should it be $\rho_{\mathrm{o}}$ or $\rho_{\mathrm{B}}$ ? The former would express the complete polaron scattering strength including both resonant and non-resonant parts relative to the situation in the absence of quantization into Landau levels, whereas the latter would give a value relative to other scattering mechanisms between the same quantized levels.

Most theories predict that $\rho_{x x}$ should be constant in field [ie. no magnetoresistance] whereas the experiments show an increase of between 2 and $50 \%$ per tesla, being most pronounced at lowest temperatures. The theory of Mori et al. (1988b) predicts some magnetoresistance but is insufficient to account for the observed behaviour. They give the non-oscillatory part of the magnetoresistance as: 


$$
\rho_{B}=\rho_{0}+2 \Delta \rho[1 / \pi] \tan ^{-1}[1 / \gamma]
$$

where $\overline{\Delta \rho}$ is given by Eqn. 2.23 and is also the prefactor to the oscillatory part. This suggests that the background magnetoresistance should show a similar variation between samples to the magnetophonon resonance amplitude. Experimentally $\rho_{\mathrm{B}} / \rho_{\mathrm{o}}$ is found to be sample independent at high temperature. Consideration of this fact in the light of the constancy of the zero field mobility portrayed in Fig. 2.8, suggests that the 'in field' mobility and hence the size of the magnetoresistance are also phonon limited. By contrast $\Delta \rho$ exhibits gross differences between samples [shown in Fig. 3.1 and discussed quantitively below]. Furthermore, Fig. 3.8 shows that $\Delta \rho$ decreases at low temperatures, just where the non-oscillatory part of the resistance changes the most. It therefore appears that this theory (exemplified by Eqn. 3.7) does not adequately account for the observed magnetoresistance, probably due to the complex way in which the dominant scattering mechanisms change with field and the difference between the resonant and non-resonant situations. Consequently any of the conclusions of Mori et al. regarding the amplitude of magnetophonon resonances must also be treated with some sceptisism.

However, with these experimental results use of $\rho_{0}$ or $\rho_{B}$ produces the same qualitative trends and only small quantitative differences. This is probably because, at the temperatures used, the electrons are non-degenerate and could be fairly well described by a classical picture with the only evidence of quantum effects being in the appearence of oscillations. In all that follows the zero-field resistivity will be used.

In some samples magnetophonon resonances were observed from more than one set of carriers. In particular G215, with its $\mathrm{n}^{-}$buffer layer showed a similar amount of bulk and $2 \mathrm{D}$ resonances. These were separated by noticing that on rotation of the sample in the magnetic field the $2 \mathrm{D}$ resonance positions move to fields given by $\mathrm{B}\left(0^{\circ}\right) / \cos \theta$ and so disappear at $\theta=90^{\circ}$, whereas the $3 \mathrm{D}$ resonances are insensitive to rotation. This angular dependence is shown in Fig. 3.7 from which the $2 \mathrm{D}$ component at $0^{\circ}$ can be extracted. By assuming the $2 \mathrm{D}$-mobility is optic phonon limited and so the same as for the other samples, the proportion of transport through the 2DEG could be estimated and hence values for $\Delta \rho$ and $\Delta \rho / \rho$ deduced. 


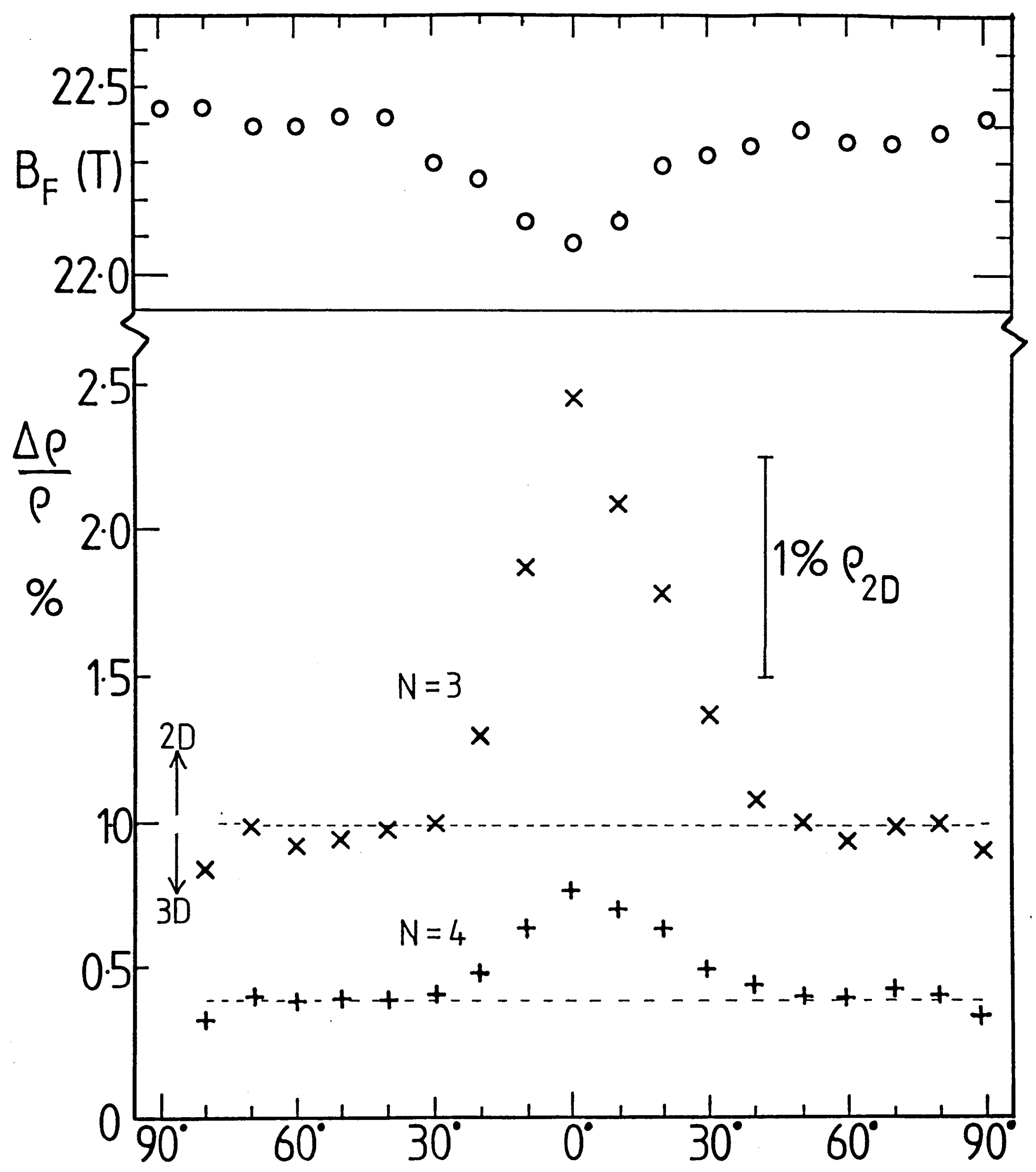

Figure 3.7 The variation of position (top) and amplitude (lower part) of the magnetophonon resonances on rotation of G215 relative to the magnetic field direction. Contributions from the 2DEG and parallel bulk conduction can be separated since the $2 \mathrm{D}$ part depends only on the $\perp$ component of field (which changes on rotation), whilst the $3 D$ part is sensetive to the total field and retains a constant amplitude. 


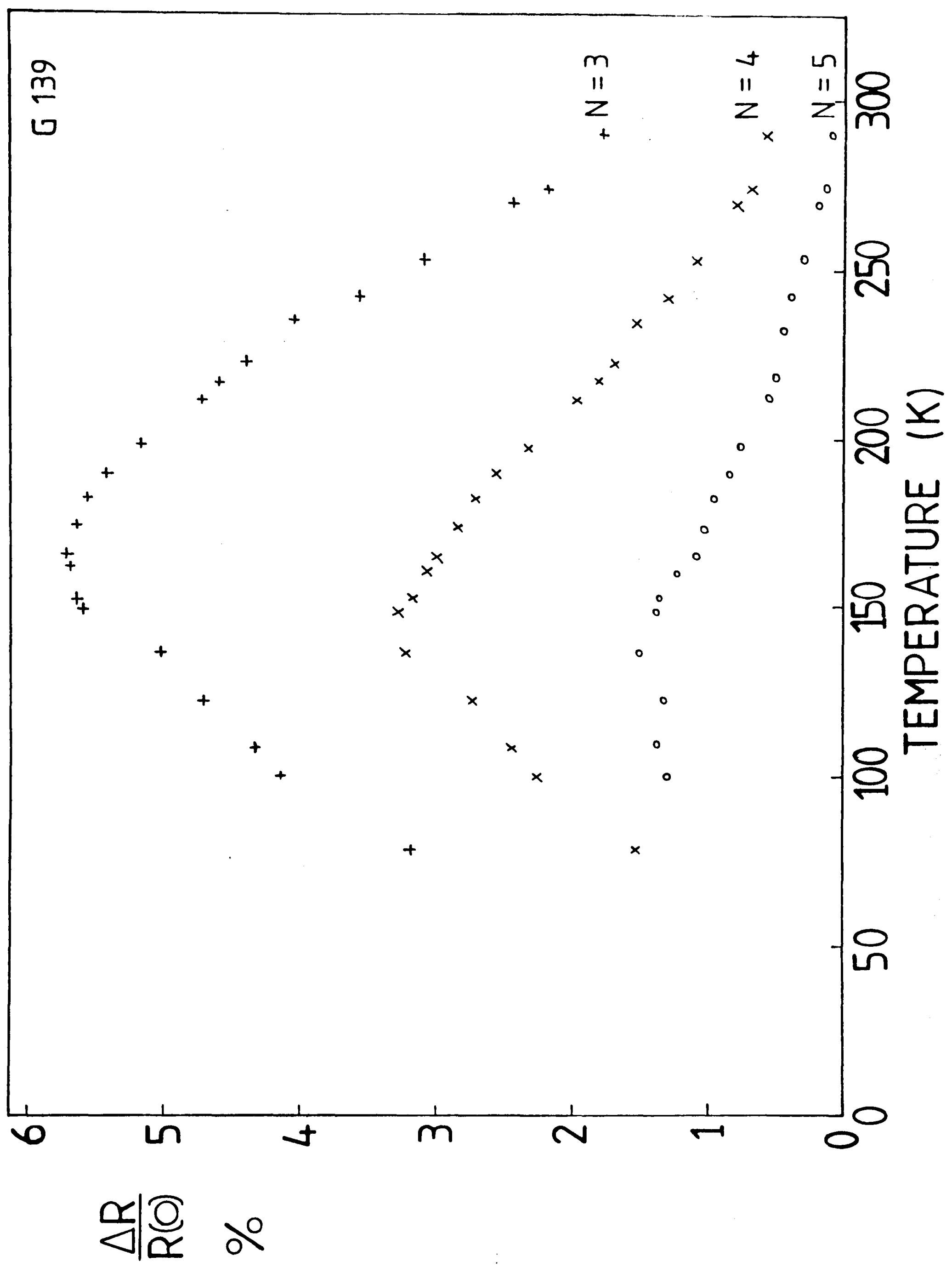

Figure 3.8 The temperature dependence of the magnetophonon resonances amplitude in two dimensions. Shown for sample G139 with $\mathrm{n}_{\mathrm{s}}=5 \times 10^{10} \mathrm{~cm}^{-2}$ relative to the resistivity at $B=0$. Notice how the maximum amplitude occurs at different temperatures for the $\mathrm{N}=3,4$ \& 5 resonances. 
The very low carrier concentration found in G161 meant conduction was poor, partly due to the problems in making contact to the 2DEG and a smaller mobility $\left(1.4 \mathrm{~V}^{-1} \mathrm{~s}^{-1} \mathrm{~m}^{2}\right)$ than the other samples $\left(\sim 2.05 \mathrm{~V}^{-1} \mathrm{~s}^{-1} \mathrm{~m}^{2}\right)$. It is expected that all samples should show the same optic phonon limited mobility at high temperatures so there is probably some parallel 3D conduction, which could well be holes in the $\mathrm{p}^{-}-\mathrm{GaAs}$. So using the mobility of bulk holes measured by Mears \& Stradling (1971) at the relevent temperature $\left(0.18 \mathrm{~V}^{-1} \mathrm{~s}^{-1} \mathrm{~m}^{2}\right.$ at $\left.180 \mathrm{~K}\right)$ and the results of Section 2.2 for two-carrier conduction the electron and hole densities can be extracted as 0.8 and $3.9 \times 10^{10} \mathrm{~cm}^{-2}$. Thus the resonance amplitudes and electron concentrations can be corrected to pure 2DEG behaviour. This sample also showed structure at $90^{\circ}$ where the resistance extrema appear inverted, and at intermediate angles the structure is very confussing. Consequently results from this sample may be misleading and so, although they will be included, not too much weight will be attached to them.

\subsubsection{Temperature Dependence}

$\Delta \rho / \rho$ is plotted as a function of temperature in Fig. 3.8 for the $N=3,4 \& 5$

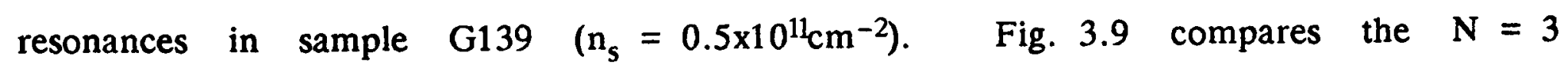
resonances in a number of samples of various carrier densities, and includes the $N=2$ resonances for G131 $\left(\mathrm{n}_{\mathrm{s}}=5.2 \times 10^{11} \mathrm{~cm}^{-2}\right)$, since in this sample $\Delta \rho / \rho<0.1 \%$ for $\mathrm{N}=3$. It can be seen that there is a maximum in the amplitudes at temperatures between $130 \mathrm{~K}$ and $200 \mathrm{~K}$ depending on the harmonic number and carrier concentration. [This is similar to the results of Englert et al. (1982b) who found a maximum at $210 \mathrm{~K}$ for the $\mathrm{N}=1$ resonance and $\sim 190 \mathrm{~K}$ for $\mathrm{N}=3$, for a sample with $\mathrm{n}_{\mathrm{s}}=3.4 \times 10^{11} \mathrm{~cm}^{-2}$.] Two factors limit this amplitude: (i) there must be a suitable population of optic phonons to cause scattering, so the amplitude decreases at low temperatures; and (ii) the Landau levels must be sufficiently well resolved to cause sharp resonances, and as the level broadening increases the amplitude will also decrease at higher temperatures. Thus there will be a maximum in the amplitude. Clearly for resonances of low harmonic number, at higher magnetic fields, the Landau levels will be further separated in energy. So the condition $\mathrm{kT}<<\hbar \omega_{\mathrm{c}}$ will hold to higher temperatures and the temperature 
Figure 3.9 Temperature dependence of the normalised $\mathrm{N}=3$ magnetophonon resonance amplitude in several samples, with the following spacer thicknesses and elecrton densities, $\left.\quad \mathrm{L}_{\mathrm{s}}(\dot{\mathrm{A}}), \mathrm{n}_{\mathrm{s}}\left(\times 10^{11} \mathrm{~cm}^{-2}\right)\right\}:-$ G139 \{1600, 0.5\}; G63 \{800, 1.5\}; G148 \{400,1.8\}; G29 \{400, 2.2\}; and G131, using the $\mathrm{N}=2$ resonance, $\{17,5.3\}$.

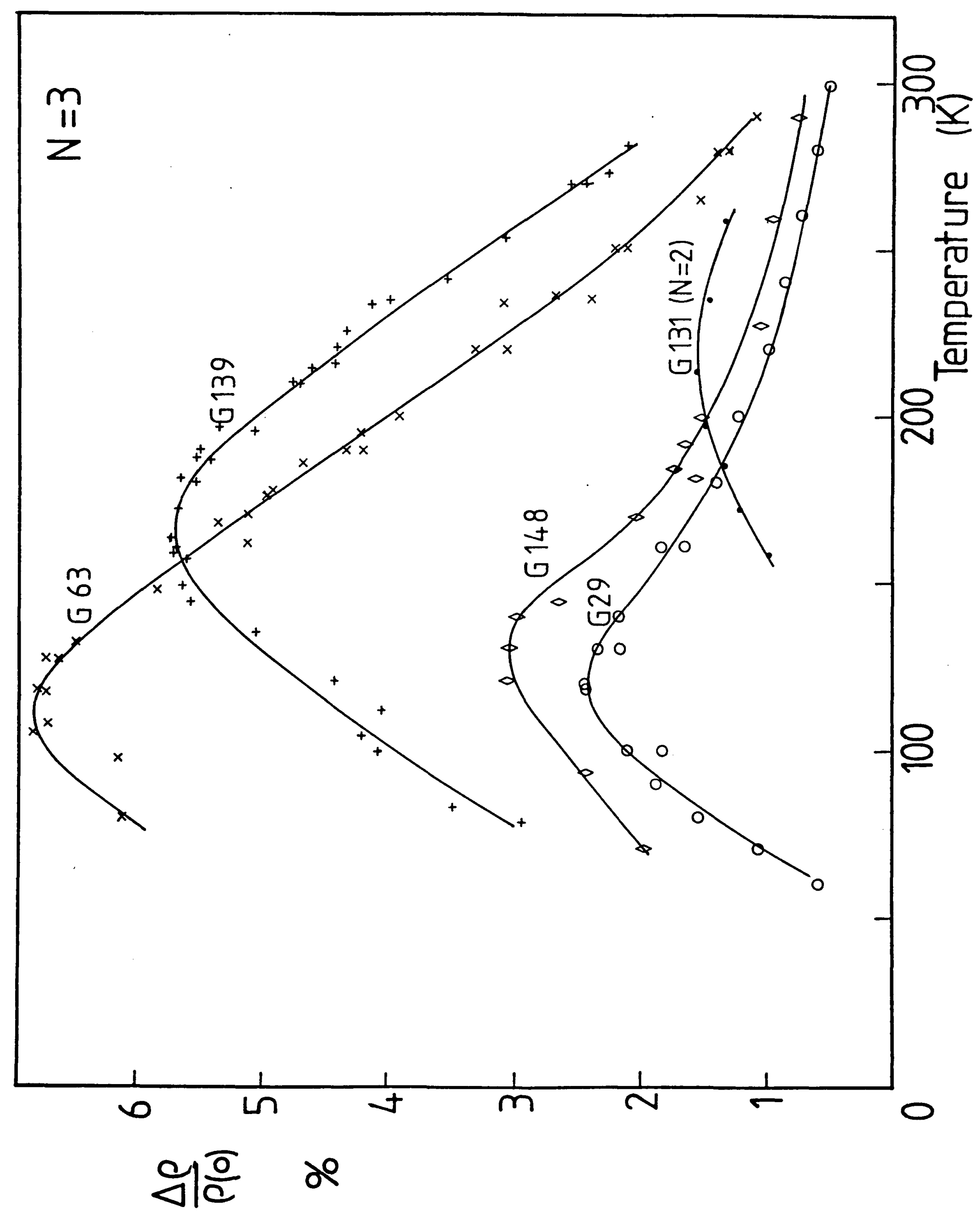


where a maximum amplitude is observed will increase. If the amplitudes are not normalised to the zero field resistivity the maximum amplitude occurs at a slightly higher temperature, as shown in Fig. 3.10 but only $\sim 30 \mathrm{~K}$ greater in contrast to the much larger differences found in bulk GaAs (Eaves et al. 1975).

In high carrier concentration samples (typically $n_{s}>3 \times 10^{11}$ ) the magnetophonon resonances may be masked by the persistence of Shubnikov-de Haas oscillations to quite elevated temperatures (Brummell et al. 1984). However, the amplitude of the latter will decrease monotonically with temperature and their positions will shift with carrier concentration in a given sample and so the shape of Figs. 3.8-10 show that we are indeed looking at magnetophonon resonances.

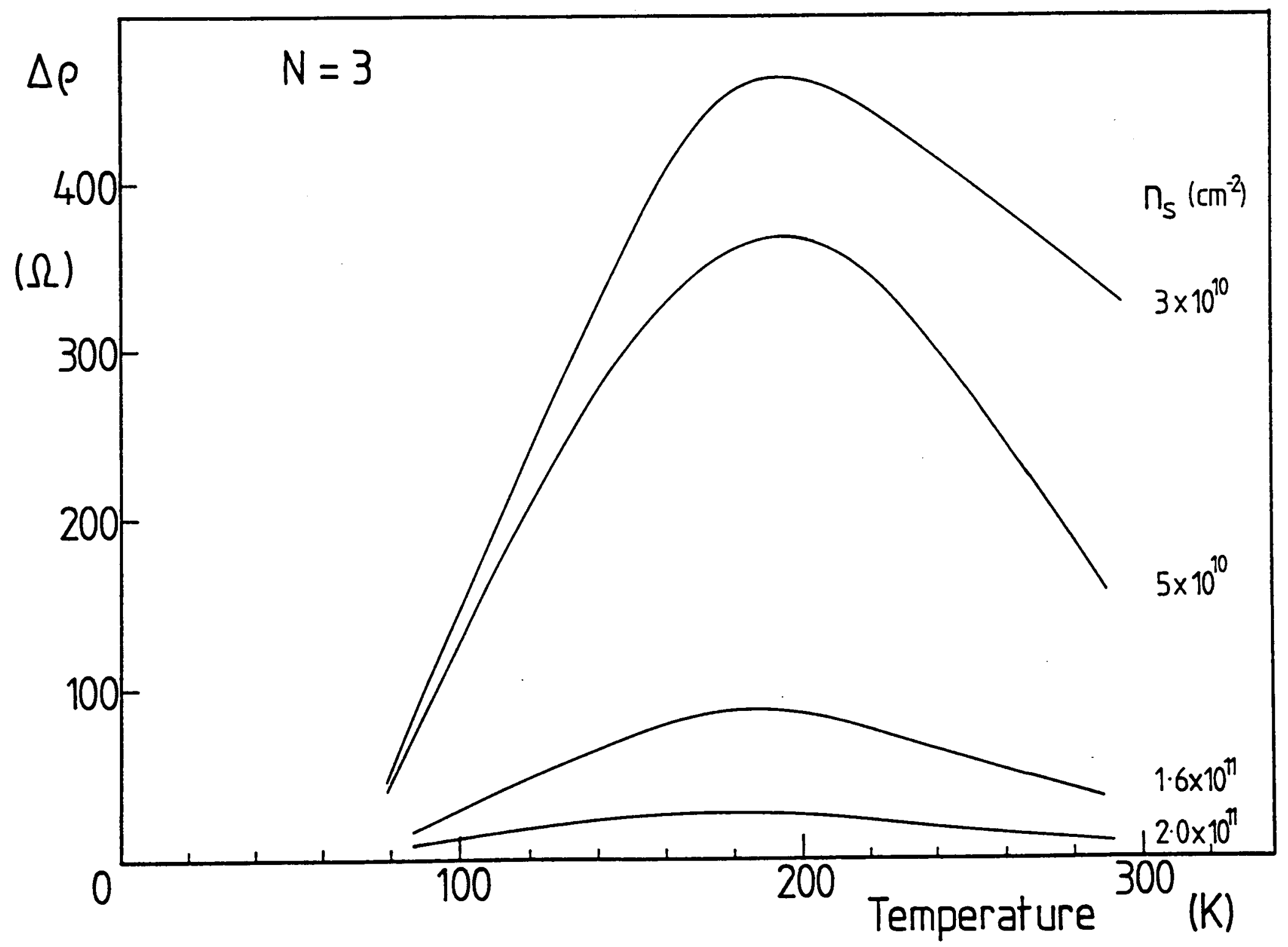

Figure 3.10 Temperature dependence of the unnormalised $\mathrm{N}=3$ magnetophonon resonance amplitude at carrier concentrations of 0.3 and $0.5 \times 10^{11} \mathrm{~cm}^{-2}$ in G139; $1.6 \times 10^{11} \mathrm{~cm}^{-2}$ in $\mathrm{G} 156$ and $2.0 \times 10^{11} \mathrm{~cm}^{-2}$ in $\mathrm{G} 29$. 


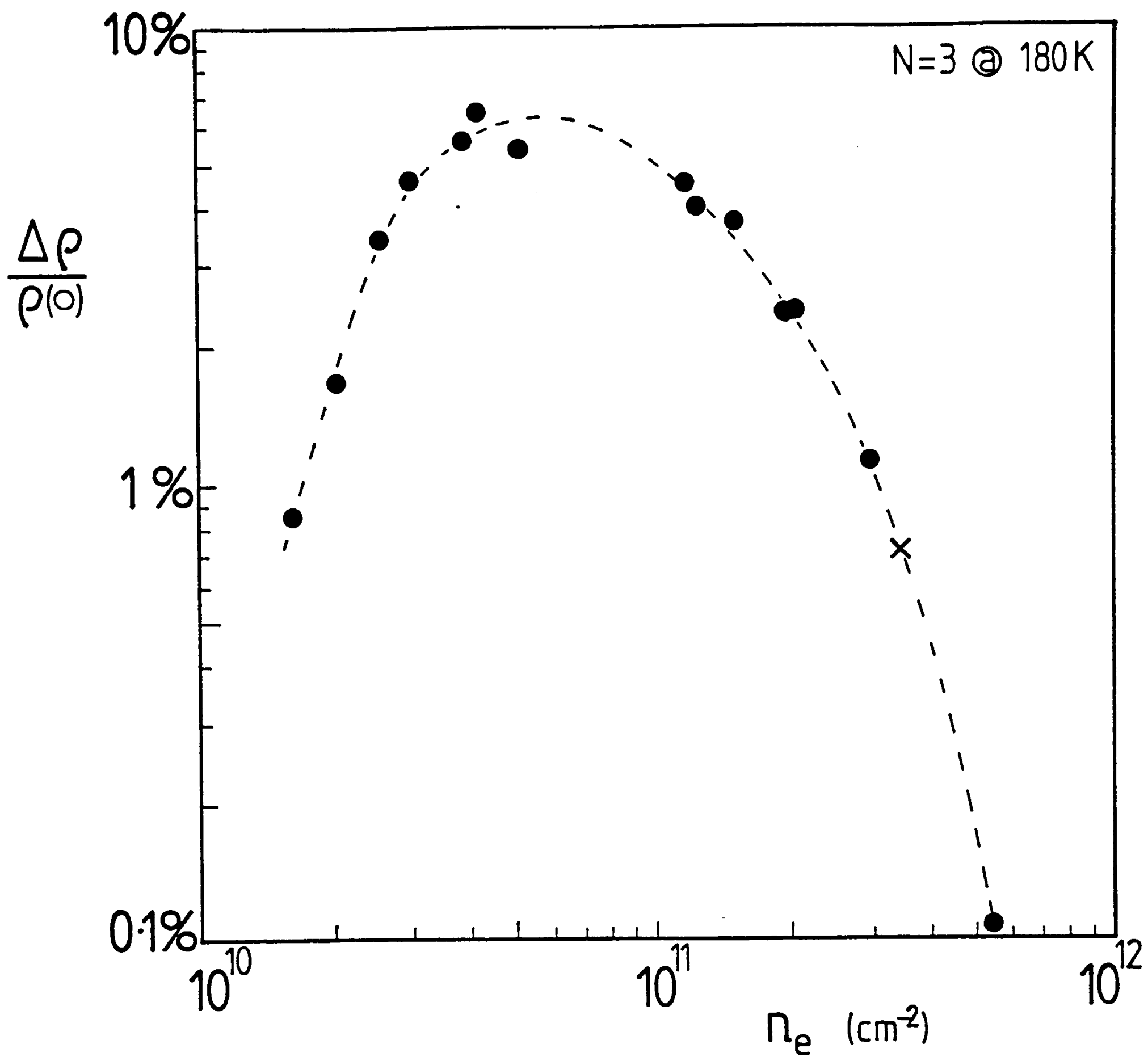

Figure 3.11 Normalised amplitude of the $\mathrm{N}=3$ resonance at $180 \mathrm{~K}$, showing a systematic dependence on carrier concentration for all the studied GaAs-GaAlAs heterojunctions, with a maximum at $\mathrm{n}_{\mathrm{s}} \sim 7 \times 10^{10} \mathrm{~cm}^{-2}$.

\subsubsection{Carrier Concentration Dependence}

The MPR amplitudes were studied in a series of samples of different spacer layers to allow the carrier concentration dependence to be determined. Within a given sample $\mathrm{n}_{\mathrm{s}}$ could also be varied by persistent photoconductivity below $\sim 120 \mathrm{~K}$, by illumination with a red LED, and to a certain extent at higher temperatures by continuous illumination. The normalised amplitudes $(\Delta \rho / \rho)$ at $180 \mathrm{~K}$ are shown in Fig. 3.11 for the $\mathrm{N}=3$ resonances, and show a systematic behaviour with similar amplitudes from all samples of a particular spacer layer thickness. This is in marked contrast to bulk results (Stradling \& Wood 1968) where the amplitude appears to be fixed by the impurity density. 


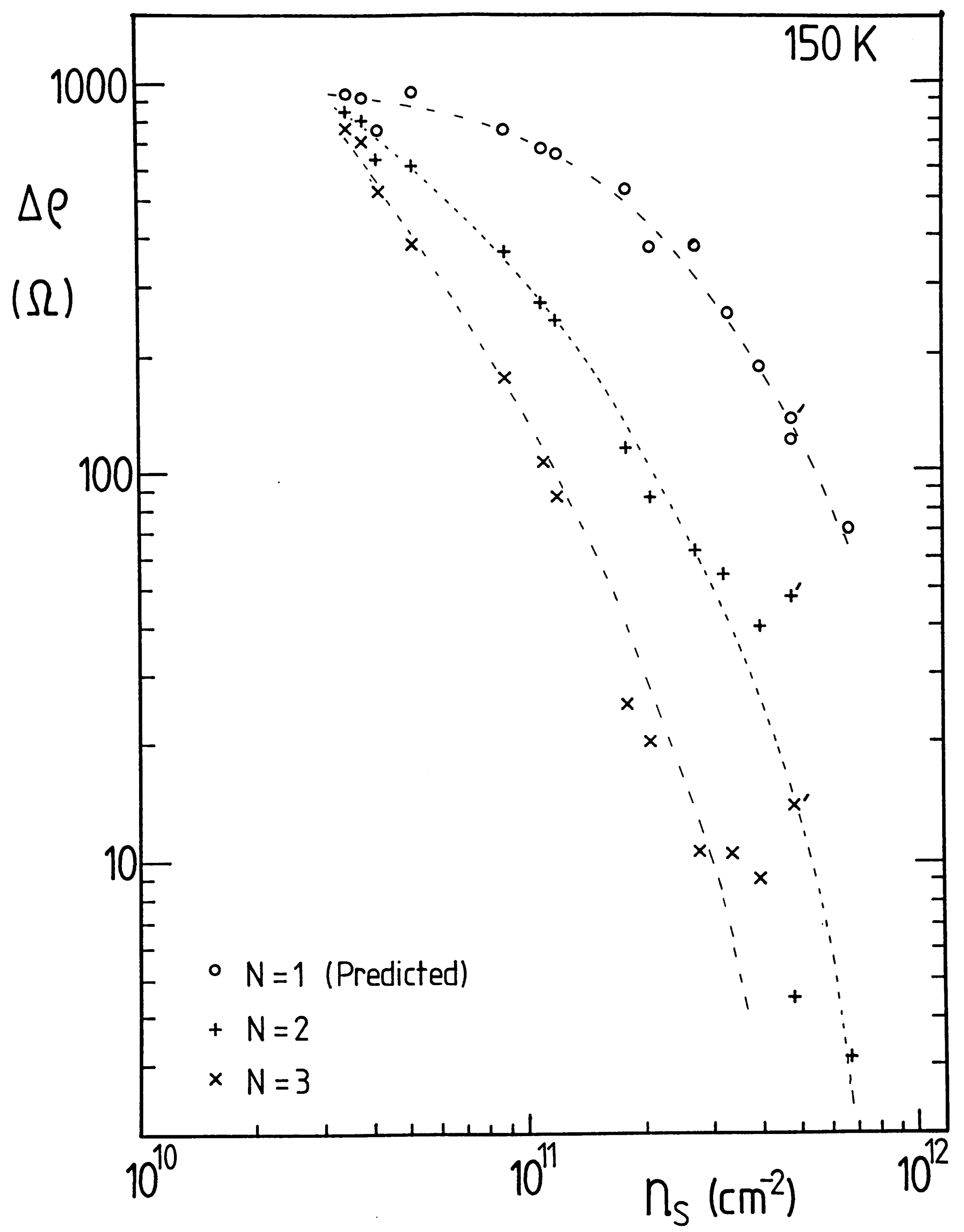

Figure 3.13 As Fig. 3.12, except the amplitudes are not normalised to the zero field resistivity. Notice how this removes the maximum (data for G161 is omitted from both figures) but leaves a qualitatively similar correlation. 
Fig. 3.12 shows data for the $\mathrm{N}=2 \& 3$ resonances at $150 \mathrm{~K}$ using the higher magnetic fields provided by the Oxford hybrid magnet. This figure also includes amplitudes of Gregoris (1985) [where the carrier concentration was varied by hydrostatic pressure] and a prediction for the size of the fundemental resonance which would appear at $\sim 23 \mathrm{~T}$, based on the amplitude of the $N=2$ peak and the measured damping factor $\tilde{\gamma}_{23}$. As $\tilde{\gamma}$ seems to decrease with field this may be slightly overestimated.

It can be seen that whilst the data for the samples in the dark follows a smooth curve with a maximum value around $n_{s}=2 \times 10^{11} \mathrm{~cm}^{-2}$, photoexcitation of carriers (shown as pairs of points connected by a dashed line) always leads to an increase in resonance amplitude. At low carrier concentrations the amplitudes rise almost linearly with $\mathrm{n}_{\mathrm{s}}$, as can be seen most clearly for the $\mathrm{N}=1$ resonance, which is where the damping of the oscillations plays the least important role.

Although the zero field mobility is optic phonon limited at the temperatures of interest and shows little change between samples, it is possible that the trends shown in Figs. 3.11 \& 3.12 are just artifacts of the ratioing process, so the unnormalised amplitudes $(\Delta \rho)$ have been plotted in Fig. 3.13. This shows the same general trend as Fig. 3.12, but with the photoexcited results now following the same curve as results in the dark and the maximum amplitude appearing at a slightly lower carrier concentration. By removing the rather questionable data from G161 in Fig. 3.13 this maximum disappears, leaving an amplitude constant at low $\mathrm{n}_{\mathrm{s}}$ and decreasing at higher densities.

It appears that the best correlation between the magnetophonon amplitude and any other factor is with the low temperature, zero-field mobility. In Fig. 3.14 the amplitude of the fundamental resonance is plotted against the mobility at $4.2 \mathrm{~K}$ for all the samples studied. The low mobility end of this curve is particularly interesting as it includes data from samples $G 161$ and G131, which have similar mobilities but very different spacer layers and carrier concentrations. These all follow the same general curve, as does G215 which up to now has always had to be treated as an exception. This is an intriguing result especially when it is considered that at the temperature where the oscillations are measured the low field mobility is independent of both carrier 
concentration and spacer thickness. Further, this observation has important practical implications as it allows a prediction of the low temperature mobility from a simple high temperature measurement, where there is otherwise no distinction between samples.

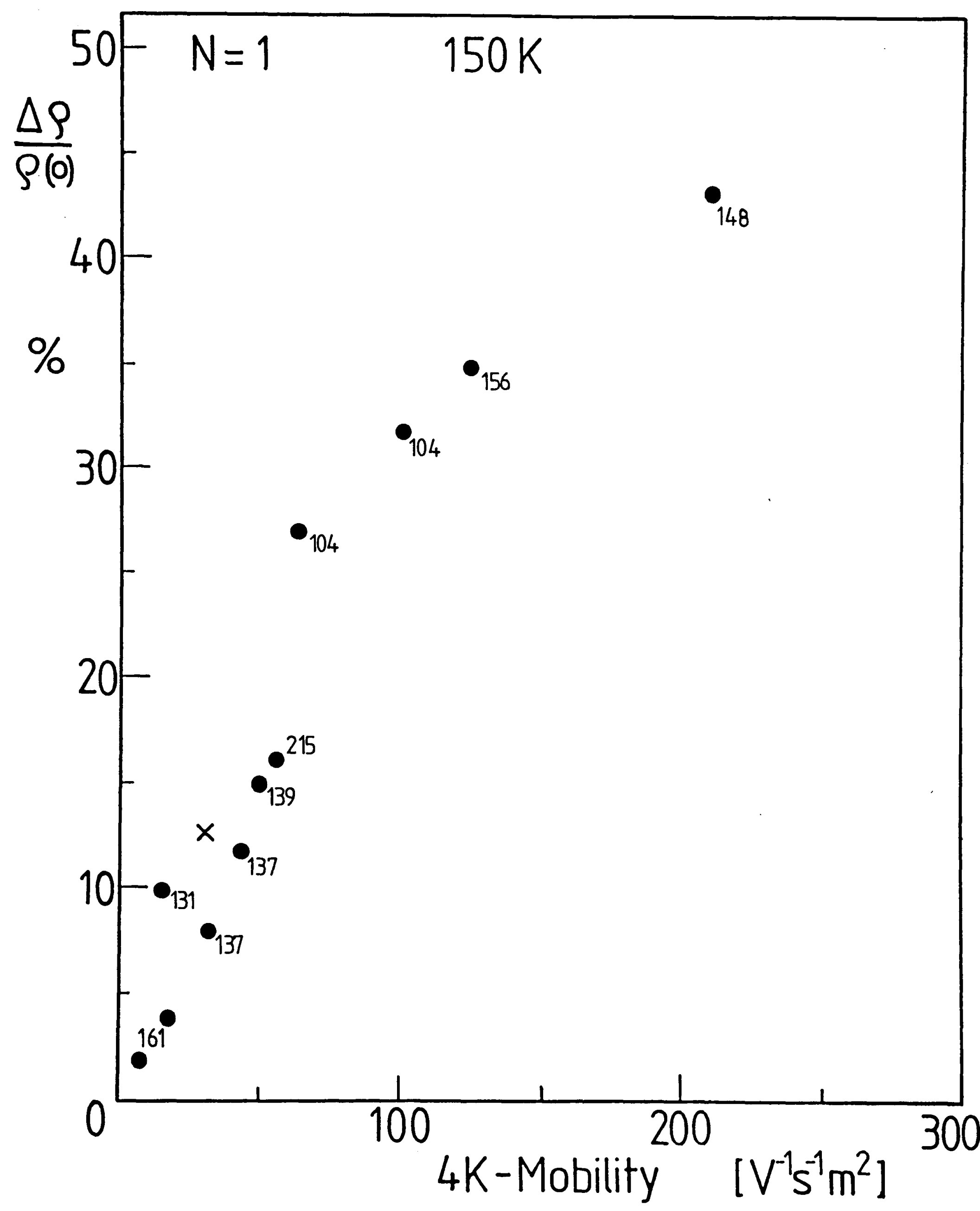

Figure 3.14 Normalised magnetophonon resonance amplitude at $150 \mathrm{~K}$ demonstrating a remarkably strong correlation with the zero field transport mobiliy measured at $4.2 \mathrm{~K}$. Note especially how samples with wildly different carrier densities and structures (eg. G131, G161 and G215) follow the same smooth trend. 


\subsubsection{Discussion of the Resonance Amplitudes}

There are two factors which influence the measured magnetophonon amplitudes: the damping of the oscillations; and the strength of the undamped coupling.

As shown in Eqn. 2.20 the damping factor is proportional to the Landau level broadening, $\Gamma$, and as we have a separate measurement of $\tilde{\gamma}$ (shown as a function of $n_{s}$ and $T$ in Figs. $3.2 \& 3.4)$ it should be possible to investigate the intrinsic coupling strengths. Initially it might be expected that an increase in broadening would inevitably lead to a reduction in coupling strength. However, the monotonic increase in $\tilde{\gamma}$ with $\mathrm{n}_{\mathrm{s}}$ would imply a similar decrease in amplitude, which is at varience with the observed maximum amplitude around $1 \times 10^{11} \mathrm{~cm}^{-2}$. From Fig. 3.12 the magnetophonon amplitude at $150 \mathrm{~K}$ can be empirically represented by:-

$$
\frac{\Delta \rho}{\rho_{\mathrm{o}}} \quad=0.02\left(\mathrm{n}_{\mathrm{s}}\right)^{3 / 2} \exp (-\mathrm{N} \tilde{\gamma})
$$

As $\tilde{\gamma}$ increases with $n_{s}$ the broadening of the levels will only dominate $\Delta \rho / \rho$ at high carrier densities, whilst at low densities the exponential will be slowly varying and the amplitude will be determined by the number of carriers present. The temperature variation of the resonance amplitudes will enter through both the numerical prefactor, which depends on the phonon occupation number and gives a reduction at low $\mathrm{T}$ and on $\tilde{\gamma}$ (through Eqn. 3.4) which will account for the fall at high temperatures.

In the course of studying the hot electron magnetophonon effect, Warmenbol et al. (1989) have investigated the influence of the broadening parameter, $\Gamma_{0}$ defined by:

$$
\Gamma=\left(2 \hbar \omega_{c} \Gamma_{0} / \pi\right)^{\frac{1}{2}}
$$

( $\Gamma_{0}$ is constant in field), on the resonance amplitudes and find a decrease like:

$$
\frac{\Delta \rho}{\rho_{0}} \propto \exp \left[\frac{\mathrm{a} N \Gamma_{0}}{\hbar \omega_{0}}\right]
$$

where $a$ is a constant depending on the electron velocity. The similarity between this and Eqn. 3.8 is clear to see. 
(i) Influence of the Damping Factor on $\Delta \rho / \rho$

The expression 3.8 suggests the level broadening is important in determining the resonance amplitudes, except at low densities, and the discussion of $\tilde{\gamma}$ in Section 3.2 shows this depends for the most part on remote impurity scattering. Mori et al. (1988b) have also calculated magnetophonon amplitudes $(\Delta \rho / \rho)$ due to remote impurity scattering and, as expected from earlier comments, find qualitative agreement at high carrier concentrations (although their predictions are a factor of $\sim 4$ too large), but fail to account for the observed decrease at lower densities.

Thus the picture at high carrier densities can be readily explained. As $n_{s}$ is increased, by narrowing the spacer layer, there will be more remote impurity scattering; therefore broader Landau levels and so a larger damping factor and also smaller resonance amplitudes. By contrast photoexcitation increases the carrier density without changing the spacer thickness, resulting in a greater resonance amplitude but no change in $\tilde{\gamma}$. This provides a confirmation that the effect on the resonance amplitudes of $n_{s}$ and of $\tilde{\gamma}$ can be decoupled. Thus, having discussed the origins of variations with the damping factor, it is now necessary to account for the intrinsic variation in amplitude with carrier density.

\section{(ii) Influence of carrier concentration on $\Delta \rho / \rho$}

By considering $\Delta \rho / \rho_{0}$, the magnetophonon amplitude may be viewed as the probability of an electron absorbing an optic phonon normalised to the scattering probability in zero field. This inelastic scattering will be proportional to the density of final unoccupied states (which decreases like $n_{s} \exp \left\{-\hbar \omega_{c} / k T\right\}$ ) and the phonon population ( $\left.\sim \exp \left\{-\hbar \omega_{o} / \mathrm{kT}\right\}\right)$. At high magnetic fields and low carrier densities the occupation of elevated Landau levels will be negligable and so the scattering probability should be independent of $n_{s}$.

The factor of $n_{s}^{3 / 2}$ appearing in Eqn. 3.8 is reminiscent of the similar factor found in the low temperature mobility, a connection which is corroborated by Fig. 3.14. However, at low temperatures this factor arrives from the density dependence of the Fermi wavevector, but at the temperatures of interest in the magnetophonon effect the 
carriers are no longer degenerate and have wavevectors to a large part determined by the thermal energy, which does not depend on $n_{s}$. Other factors affecting $\mu_{4 \mathrm{~K}}$ are: spacer layer thickness, which we have just seen determines the remote impurity scattering responsible for limiting $\Delta \rho$ at large $n_{s}$; background impurities - these are important at low carrier densities where the impurity potential cannot be effectively screened, but at high temperatures the screening should never be as effective. [However it must be mentioned that some cyclotron resonance results suggest screening of background impurities may be important at elevated temperatures (Hopkins et al. 1989 and refs. therin).] So although the low temperature mobility is a good predictor of magnetophonon resonance amplitudes, it is not clear exactly why this should be; unless screening and occupation effects play a more important role at high temperatures than previously expected.

At $180 \mathrm{~K}$ the system should be close to degenerate statistics at approximately $3 \times 10^{11} \mathrm{~cm}^{-2}$, where we have $\mathrm{kT} \approx \hbar \omega_{c} \approx \mathrm{E}_{F}(\mathrm{~B}=0)$. Above this point we might expect screening to assume a more important role, and the relative importance of transitions occuring between higher Landau levels will increase. It should be remembered that while the statistics remain non-degenerate there is nearly no change in the relative contribution to the resistivity from the different Landau level transitions, and so the carrier concentration dependence of the amplitude at very low densities can only be associated with some modification of the basic electron-phonon interaction.

\section{Effects of Carrier Concentration on Polaron Coupling}

At resonance the polaron coupling will be determined by the combined process of emission and absorption of virtual LO phonons within the phonon coherence time. This process should be independent of temperature, since there are no restrictions on the numbers of virtual phonons. At first sight it should also be independent of $n_{s}$, as every electron absorbing a phonon will leave hole behind in the orginal level for the subsequent emission process. This hole would have both the required $\mathbf{k}$ value and would also be in the same place as the electron in real space since the electron will have hardly travelled any distance during the phonon coherence time. 
In cyclotron resonance measurements near to the resonant polaron region, Langerak et al. (1988) found the strength of polaron coupling was similar in $2 \mathrm{D}$ to that in bulk at low densities, but was almost completely suppressed at high $n_{s}$. In their calculations Peeters \& Devreese consider virtual transitions between the same Landau level and sum over all the initial and final states including a factor of $f_{e}\left(1-f_{e}\right)$, [with $f_{e}$ being the Fermi factor for electrons in that level]. This gives rise to a prediction that the occupation effects suppress polaron coupling at even filling factors and that since the quantum limit is reached earlier for low carrier density samples, these will show stronger polaron coupling. However, this argument seems to be incompatible with the foregoing intuitive picture. An alternative explaination may be that it would be possible to renormalise the many-electron wavefunction in the lower level within the phonon coherence time ( $\sim 7 \mathrm{ps,} \mathrm{compared} \mathrm{to} \mathrm{an} \mathrm{electron} \mathrm{momentum} \mathrm{relaxation} \mathrm{time} \mathrm{of} \sim 1 \mathrm{ps})$, thus removing the convenient hole and meaning that the densities of final electron states would need to be considered for the phonon emission process. Since absorption is only allowed if subsequent emission can also occur, this would reduce the coupling as the carrier density increased.

Initially one might expect the magnetophonon amplitudes to decrease at high carrier concentrations if the coupling is weaker. However, if we are concerned with a truly resonant process, any strength of electron-phonon coupling would give an infinite magnetophonon amplitude, and the observed amplitude would depend on the other scattering mechanisms which broaden the levels. Indeed calculations without additional broadening mechanisms in $2 \mathrm{D}$ systems show $\delta$-function singularities at the resonance condition (Lassnig \& Zawadski 1983). Thus, as $n_{s}$ increases the level broadening by virtual phonon exchange will be smaller and hence the magnetophonon resonance amplitude should be larger.

This is supported by experimental observations that some of the strongest bulk magnetophonon oscillations are found in the weakly polar InSb $-\alpha \sim 0.02$ [Fujisawa 1978 found $\Delta \rho / \rho=12 \%$ for $N=3$ ], whereas nothing at all is seen in the alkali halides where $\alpha \sim 3$ (Harper et al. 1973). However, the optic phonon limited 
mobility at $\sim 150 \mathrm{~K}$ is $<0.01 \mathrm{~V}^{-1} \mathrm{~s}^{-1} \mathrm{~m}^{2}$ in $\mathrm{KBr}$ (Ahrenkiel \& Brown 1964) compared to $\sim 20 \mathrm{~V}^{-1} \mathrm{~s}^{-1} \mathrm{~m}^{2}$ in InSb. The most polar semiconductor in which the magnetophonon effect has been observed is CdTe $-\alpha=0.3$, where values of $\Delta \rho / \rho$ upto $1 \%$ have been observed by Mears et al. (1968) in samples with mobilities of $\sim 0.3 \mathrm{~V}^{-1} \mathrm{~s}^{-1} \mathrm{~m}^{2}$. This suggests that it is perhaps the mobility that determines the magnetophonon resonance amplitude, and that in the relevent temperature range $\mu$ is optic phonon limited and hence determined by $\alpha$. In the present case $\Delta \rho / \rho \sim 10 \%$ with $\mu$ taking the same value in each sample of $\sim 3 \mathrm{~V}^{-1} \mathrm{~s}^{-1} \mathrm{~m}^{2}$. The constancy of $\mu$ does not rule out a change in the effective $\alpha$, since $\mu$ is measured in zero field and the proposed change in coupling strength is due to occupation effects, which are only manifested in quantising magnetic fields.

Unfortunately, by this same arguement, as with any based on level broadening, the damping factor would be expected to decrease with increasing $\mathrm{n}_{s}$, contrary to the experimental observations! However, we have just shown that $\tilde{\gamma}$ is dominated by remote impurity, not phonon, scattering. So it may be that as the electron-optic phonon coupling strength decreases (ie. $\mathrm{n}_{\mathrm{s}}$ is increased, at fixed spacer thickness) the broadening of the hybrid Landau level + phonon state decreases - enhancing the magnetophonon amplitude - without noticably changing the Landau level broadening - leaving $\gamma$ unaltered.

\section{Enhancement by Coherent Phonon Absorption}

Up to now we have only been considering the absorption of single phonons and have specified the likelihood of such an event by the occupation number, $\mathrm{N}_{0}$. At some time later, which for this arguement has to be after the phonon system has re-thermalised, the electron will emit a phonon and return to the lower Landau level. In so doing the electron will scatter to another cyclotron orbit and change its centre coordinate (ie. recoil). To conserve momentum this requires the wavevector of the emitted phonon to be in the plane of the 2DEG, leaving the phonon in just the right place to be re-absorbed by another electron (the dispersion curve for optic phonons is very flat so they hardly travel any distance during their lifetime) giving rise to a 
coherent absorbtion process. This could be pictured as increasing the phonon occupancy in the region of the $2 \mathrm{DEG}$ by $\delta \mathrm{N}$ - proportional to the electron concentration and the phonon population. The probability of absorbing a phonon then becomes $a\left(N_{0}+\delta N\right)$, and $\delta N=b n_{s} \cdot a\left(N_{o}+\delta N\right)$. Dealing with this self consistently:

$$
\delta N=\frac{a b n_{s}}{1-a b n_{s}}
$$

$\therefore$ Prob. of absorption $\propto \quad \frac{\Delta \rho}{\rho} \propto \frac{\mathrm{aN}_{0}}{1-\mathrm{abn}_{s}}$

Thus the magnetophonon resonance amplitude would increase with $n_{s}$ due to coherent phonon absorbtion. This description is just intended to point out the possible importance of the direction of the emitted phonon's wavevector on the magnetophonon amplitude. Generally, the stronger the recoil the more peaked in the forward direction will the phonon distribution be, but a full $q$ dependent calculation is required to make any detailed predictions.

\subsection{MAGNETOPHONON EFFECTIVE MASS}

Magnetophonon resonance may be used to deduce phonon frequencies from the resonance positions using Eqn. 3.1, but it is first necessary to have an accurate value of the relevent effective mass. The magnetophonon effective mass, $m_{M P R}^{*}$ must be adjusted from the band edge value, $\mathrm{m}_{\mathrm{o}}^{*}$, to take account of the non-parabolicity of the conduction band and polaron coupling.

\section{$\underline{3.4 .1 \quad \text { Non-parabolicity }}$}

The deviations from a parabolic conduction band discussed in Section 1.4 mean that the effective mass will be larger than $\mathrm{m}_{\mathrm{o}}^{*}$ for transitions above the band edge. Using k.p theory the enhanced mass relevent to magnetophonon resonance in a heterojunction is, using Eqn. 1.26:

$$
\frac{1}{\mathrm{~m}_{\mathrm{MPR}}^{*}}=\frac{1}{\mathrm{~m}_{\mathrm{o}}^{*}}\left\{1+\frac{\mathrm{K}_{2}}{\mathrm{E}_{\mathrm{g}}}\left[(2 \overrightarrow{\mathrm{L}}+\mathrm{N}+1) \frac{\hbar \omega_{\mathrm{LO}}}{\mathrm{N}}+2\left\langle\mathrm{~T}_{\mathrm{z}}\right\rangle\right]\right\}
$$

where $E_{g}$ is the band gap, $\bar{L}$ is the mean quantum number of the initial Landau level calculated from the Fermi distribution at the particular temperature and occupancy, $\left\langle T_{Z}\right\rangle$ 
is the kinetic energy due to motion perpendicular to the layers.

Accurate calculation of $\overline{\mathrm{L}}$ and $\left\langle\mathrm{T}_{\mathrm{z}}\right\rangle$ would only be possible if the exact band structure and the shape of the confining potential were well known. For this reason it is much easier to measure an effective mass from far infra-red cyclotron resonance and then to apply some small corrections to account for the differences in the electronic transitions between these two processes. Again using k.p theory the cyclotron mass, $\mathrm{m}_{\mathrm{CR}}^{*}$, is given by:

$$
\frac{1}{\mathrm{~m}_{\mathrm{CR}}^{*}}=\frac{1}{\mathrm{~m}_{\mathrm{o}}^{*}}\left\{1+\frac{2 \mathrm{~K}_{2}}{\mathrm{E}_{\mathrm{g}}}\left[(\overline{\mathrm{L}}+1) \hbar \omega_{\mathrm{c}}+\left\langle\mathrm{T}_{\mathrm{z}}\right\rangle\right]\right\}
$$

As previously discussed a value of $\mathrm{K}_{2}=-1.4$ will be used.

\subsubsection{Polaron Coupling}

The condition for magnetophonon resonance requires the $L+N^{\text {th }}$ Landau level to be degenerate with the $L^{\text {th }}$ level plus one optic phonon, ie the case of resonant polaron coupling, so the full, field-independent, resonant correction to the magnetophonon mass must be used and not the field-dependent non-resonant correction. This means that in finding $\mathrm{m}^{*}{ }_{\mathrm{MPR}}$ from $\mathrm{m}^{*} \mathrm{CR}$ the non-resonant polaron contribution to the cyclotron resonance mass must first be subtracted, using $\mathrm{K}_{2}^{\mathrm{pol}}=-0.4$.

In bulk semiconductors, resonant polaron effects introduce an additional enhancement of the magnetophonon mass by a factor of $1+\alpha / 4$ (Harper et al. 1973), but the magnitude of this correction in 2-D systems is not known.

The polaron coupling in 2-D has been predicted to be stronger due to the more singular densities of states (Das Sarma 1984; Larsen 1984; Peeters et al. 1986), but may be reduced by the finite extent of the wavefunction perpendicular to the interface (Das Sarma 1983; Larsen 1984; Lassnig \& Zawadzki 1984b) and the effects of occupancy and screening (Das Sarma \& Mason 1985; Sigg et al. 1985; Peeters et al. 1988). Exact calculations have only been made for corrections to the cyclotron resonance position by use of the dynamical conductivity in a memory function approach (Wu et al. 1987), from which it would be difficult to extract the correction required for the magnetophonon mass. 
Early experimental work showed coupling strengths reduced (Sigg et al. 1985; Singleton et al. 1986a; Hopkins et al. 1986; Horst et al. 1985; Zeismann et al. 1987), similar (Singleton et al. 1988) and enhanced (Singleton et al. 1986a; Horst et al. 1983) compared to that in bulk systems, but more recently Langerak et al. (1988) have shown a coupling which depends on the carrier concentration, being comperable to the effect in bulk at low $n_{s}$ and becoming much smaller at high densities due to occupation effects. However at elevated temperatures, where the system is not always degenerate, these effects will be less important, as seen in the mass dressing observed in the non-resonant region by Brummell et al. (1987a) above 100K. Barnes et al. (1989) have also investigated the temperature dependence of $\mathrm{m}^{*} \mathrm{CR}$, but closer to the resonant polaron region, and find larger changes for high carrier concentration samples suggesting that more resonant polaron coupling is restored as the screening is removed, although this may not be as much as the bulk value. Brummell et al. used a correction of $1+3 / 16^{\alpha}$ which is $75 \%$ of the $3 \mathrm{D}$ case and as a reasonable comprimise. Bearing in mind the recent results of Langerak et al. and Barnes et al. the correction could be even more than the $3 \mathrm{D}$ value and so $\mathrm{I}$ will use $1+\alpha / 4$ and note that this may be an over estimate for high density samples and an under estimate for low density samples.

\subsubsection{Calculation of Magnetophonon Mass}

At a given temperature and magnetic field the effect on the magnetophonon and cyclotron masses due to band non-parabolicity will be the same. So, once the non-resonant polaron contribution to $\mathrm{m}^{*} \mathrm{CR}$ has been removed, the only corrections necessary are to account for the different weightings of initial Landau level, using $K_{2}=-1.4$ to account for the band non-parabolicity. This gives:

$$
\frac{1}{\mathrm{~m}_{\mathrm{MPR}}^{*}}=\frac{1}{\mathrm{~m}_{\mathrm{CR}}^{*}}+\frac{\mathrm{K}_{2} \hbar \omega_{\mathrm{LO}}}{\mathrm{m}_{\mathrm{o}}^{*} \mathrm{E}_{\mathrm{g}}}\left[1-\frac{1}{\mathrm{~N}}\right]
$$

Unfortunately, cyclotron resonance measurements have rarely been performed at exactly the required magnetic field and can never be made at the $N=1$ resonance, which must be in the Reststrahlen band. To allow for these problems $\mathrm{m}^{*} \mathrm{CR}$ was measured as 
a function of temperature using a particular wavelength, $\lambda$, and then corrected to the required magnetic field by using the experimental frequency dependence of $\mathrm{m}^{*} \mathrm{CR}$ measured by Hopkins $(1987)$ as $m^{*}{ }_{C R}=m^{*}{ }_{0}(1+0.003 B)$ in place of Eqn. 3.13 and then using Eqn. 3.15 with $(1-1 / N)$ replaced by $\left(1-B / B_{F}\right)$ we find:

$$
\mathrm{m}^{*} \mathrm{MPR}_{\mathrm{PR}}(\mathrm{T}, \mathrm{B})=\mathrm{m}_{\mathrm{CR}}^{*}(\mathrm{~T}, \lambda)\{a+b \mathrm{~B}\}
$$

where for $\lambda=118 \mu \mathrm{m}$ the constants $a$ and $b$ are $1.014 \& 0.0018$ respectively. The field dependence of the cyclotron resonance position was measured at $4 \mathrm{~K}$ and so the statistics are degenerate, which means Eqn. 3.16 will only be correct at high temperatures in the extreme quantum limit. More usually at the temperatures used for magnetophonon experiments $\mathrm{kT} \sim \mathrm{E}_{\mathrm{F}}$ which means the system is not degenerate and so the field dependence of the mass will be less than the $4 \mathrm{~K}$ result. In practice it will be $\sim 2 / 3$ which would mean using $a=1.020 \& b=0.0007$.

The result of these corrections for non-parabolicity is an enhancement of the magnetophonon mass, over that measured from cyclotron resonance at the same temperature, of $2.3 \%$ at high $\mathrm{N}$ increasing to $2.8 \%$ for $\mathrm{N}=2$ and $3.7 \%$ for $\mathrm{N}=1$. The resonant polaron correction will further enhance the mass by $\sim 1.8 \%$ for all $\mathrm{N}$.

The cyclotron mass was not measured as a function of temperature in all the samples, but was extrapolated according to the experimental $n_{s}$ dependence measured by Hopkins (1987):

$$
\mathrm{m}^{*}{ }_{\mathrm{CR}}=\mathrm{m}_{\mathrm{o}}^{*}\left\{1+\mathrm{cn}_{\mathrm{s}}\right\}
$$

At $4 \mathrm{~K} c=0.009$ if $\mathrm{n}_{\mathrm{s}}$ is measured in units of $10^{11} \mathrm{~cm}^{-2}$, but at higher temperatures the system will no longer be degenerate and so $2 / 3$ of the this value has been used (ie. $c=0.006$ ). The only sample this may cause trouble in is G131 where the carrier concentration is much larger than in other samples.

\subsubsection{Upper subband Contributions}

Finally there remains the possibility of magnetophonon resonances arrising from electrons in upper subbands in which case a weighted average of the masses in each subband should be used. In Chapter 6 the separation between the lowest two electric subbands is found to lie in the range $10-20 \mathrm{meV}$, which means there will be 
considerable upper subband population for all temperatures above $\sim 100 \mathrm{~K}$. Electrons in the upper subband will be near the bottom of the band $(k \approx 0)$ and $\left\langle T_{z}\right\rangle$ will be smaller than in the lowest subband due to the larger spread of the upper subband wavefunction. This means the mass in the upper subband will be lower than in the ground subband.

For example, cyclotron resonance measurements on G215 at low temperatures (Barnes et al. 1989b) show an additional resonance appearing after illumination of the sample (population of the upper subband), which is much weaker than the original resonance and is seen at lower magnetic field. These resonance positions correspond to masses of $0.07136 \mathrm{~m}_{\mathrm{e}}$ and $0.06878 \mathrm{~m}_{\mathfrak{e}}$, for the ground and excited subbands respectively. To calculate the required $\mathrm{m}^{*}$ MPR an appropriate weighting of the subband populations would be applied to the mass from each subband. For low carrier concentration samples at high temperature this will simply be the Boltzmann factor $\exp \left(-\mathrm{E}_{01} / \mathrm{kT}\right)$, but with more carriers present the Fermi energy may lie between the subbands and so it will be necessary to apply Fermi-Dirac statistics. However, by measuring $\mathrm{m}^{*} \mathrm{CR}$ at the required temperature (the two resonances are not resolved in the magnetophonon temperature regime) any upper subband contribution should be accounted for, as there will be the same distribution of electrons.

The previous statement must be qualified in that it requires the upper subband contribution to cyclotron resonance to be the same as experienced in magnetophonon resonance. For cyclotron resonance the optical oscillator strength will just depend on the total numbers of carriers present in each subband, whereas for magnetophonon resonance, being a transport phenomenum, it is the relative contributions to the conductivity that are important. It will be shown in Chapter 6 that for Shubnikov-deHaas oscillations from multiple subbands the total oscillatory part of the resistance is like $\Delta \rho / \rho=\Delta \rho_{1} / \rho_{1}+\Delta \rho_{2} / \rho_{2}$, producing comperable contributions from each subband, with their relative importances depending on the mobility, as opposed to the carrier concentration, in each subband. In Chapter 7 the electron mobility in each subband, relevent to both transport and cyclotron resonance type situations, are discussed and 
found not to be as simply related as might be expected. Thus there is the possibility that different weightings should be applied in calculating $\mathrm{m}^{*}{ }_{\mathrm{MPR}}$ and $\mathrm{m}^{*} \mathrm{CR}$, although one would expect the overall change in $\mathrm{m}^{*}{ }_{\mathrm{MPR}}$ to be much less than the difference of $4 \%$ between the masses for the two subbands.

\subsection{RESONANCE POSITIONS AND PHONON FREQUENCIES}

\section{$\underline{3.5 .1 \quad \text { Peak Shifts }}$}

Before anything can be said about the energies at which the electron-phonon couplings are occuring it is necessary to know how the maximum amplitude measured in the resistivity corresponds to the resonance condition 3.1. As well as changing the oscillation amplitude the damping will cause a shift of the measured extrema. Let us first examine the case for Lorentzian broadened levels and the empirical results from bulk semiconductors. Differentiation of Eqn. 2.22 (or 2.19) shows that maxima should occur when

$$
\tan \left(2 \pi \omega_{\mathrm{o}} / \omega_{\mathrm{c}}\right) \quad=-\tilde{\gamma} / 2 \pi
$$

This was studied in detail by Blakemore \& Kennewell (1975) who tabulated values of the shift for each harmonic (reproduced in Nicholas 1985) both for direct and double differentiated recordings. The resonances all move towards higher magnetic fields - a typical shift for $\tilde{\gamma}=1.5$ being $\sim 4 / \mathrm{N} \%$ for the $\mathrm{N}^{\text {th }}$ harmonic on a direct trace.

Now compare the results of Warmenbol et al. (1988). Eqn. 2.26 predicts peak positions shifted to lower magnetic fields, with the fudemental field being found from the approximate expression:

$$
\mathrm{B}_{\mathrm{F}} \quad=\quad \mathrm{B}_{\mathrm{N}}\left[\mathrm{N}+\frac{\Gamma_{0}}{2 \pi \mathrm{kT}}\right]
$$

where $B_{N}$ is the position of the $N^{\text {th }}$ maximum and $\Gamma_{0}$ is their constant broadening parameter (Eqn. 3.9). Addition of the constant background increases $\rho_{x x}$ between the resonances and shifts the peaks further to lower magnetic fields. Using a $25 \%$ background the density of states appears very similar to Lorenzian broadened levels and the peak shifts are comparable in magnitude to those predicted by Eqn. 3.18 but in the opposite direction. 
In order to decide which way the peaks have shifted the product $\mathrm{NB}_{\mathrm{N}}$ could be compared for peaks of high $\mathrm{N}$, which will only show a small shift, and low $\mathrm{N}$, where any change is most evident. In practice only two or three peak positions could be reliably obtained, and these only to $\pm 0.1 \mathrm{~T}$ which was insufficient to establish a general trend. More conclusive data would arrise from study of the fundemental resonance, requiring magnetic fields in excess of $30 \mathrm{~T}$ to see the whole resonance. Consequently a correction due to the damping factor will not be applied in determining the resonance position, but it will be remembered that this may lead to uncertainties of $\pm 4 \mathrm{~cm}^{-1}$ in the phonon frequencies deduced.

\subsubsection{The Measured Values}

The resonance positions, $\mathrm{B}_{\mathrm{N}}$, were carefully measured for each peak at a variety of temperatures and carrier concentrations. Within the limits of experimental uncertainty the same value for the product $\mathrm{NB}_{\mathrm{N}}$ was found for all $\mathrm{N}$, so it was not possible to determine the direction of peak shift discussed above. The values of $3 \mathrm{~B}_{3}$ measured at $150 \mathrm{~K}$, but not corrected for damping, are tabulated in Table 3.1 together with the masses used to calculate the apparent optic phonon frequencies from Eqn. 3.1.

\begin{tabular}{|l|l|l|l|}
\hline Sample & $\begin{array}{c}3 \mathrm{~B}_{3} \\
\mathrm{~T}\end{array}$ & $\begin{array}{c}\mathrm{m}^{*} \mathrm{MPR} \\
\times 10^{-5} \mathrm{~m}_{\mathrm{e}}\end{array}$ & $\begin{array}{c}\omega_{0} \\
\mathrm{~cm}^{-2}\end{array}$ \\
\hline G161 & 22.3 & 7263 & 287 \\
G139 & 22.2 & 7270 & 285 \\
G141 & 22.25 & 7302 & 285 \\
G156 & 22.05 & 7329 & 279 \\
G 63 & 22.2 & 7329 & 281 \\
G148 & 22.15 & 7370 & 282 \\
G 83 & 22.0 & 7370 & 280 \\
G 29 & 22.45 & 7370 & 285 \\
G 71 & 22.05 & 7358 & 280 \\
G104 & 22.5 & 7414 & 283 \\
G131 & 23.4 & 7502 & 286 \\
\hline
\end{tabular}

Table 3.1

These variations of resonance position and apparent phonon frequency with carrier concentration are illustrated in Figs. 3.15 and 3.16 respectively. It is clear from these pictures that (i) the resonance positions correspond to phonon frequencies well below 


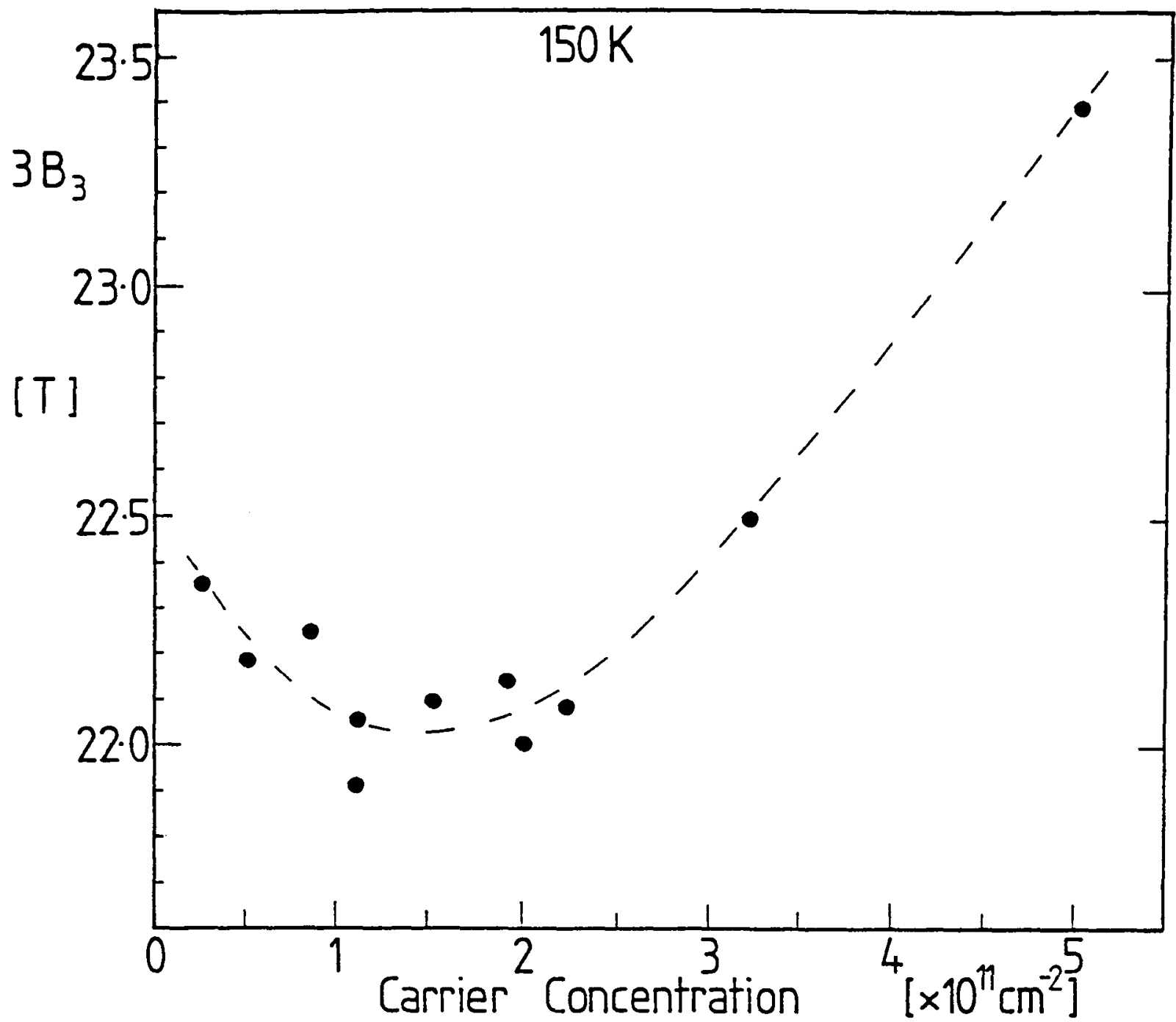

Figure 3.15 Change in the position of the $\mathrm{N}=3$ magnetophonon resonance as a function of carrier concentration, with no corrections applied, showing a strong minmum at $\mathrm{n}_{\mathrm{s}} \sim 1.5 \times 10^{11} \mathrm{~cm}^{-2}$.

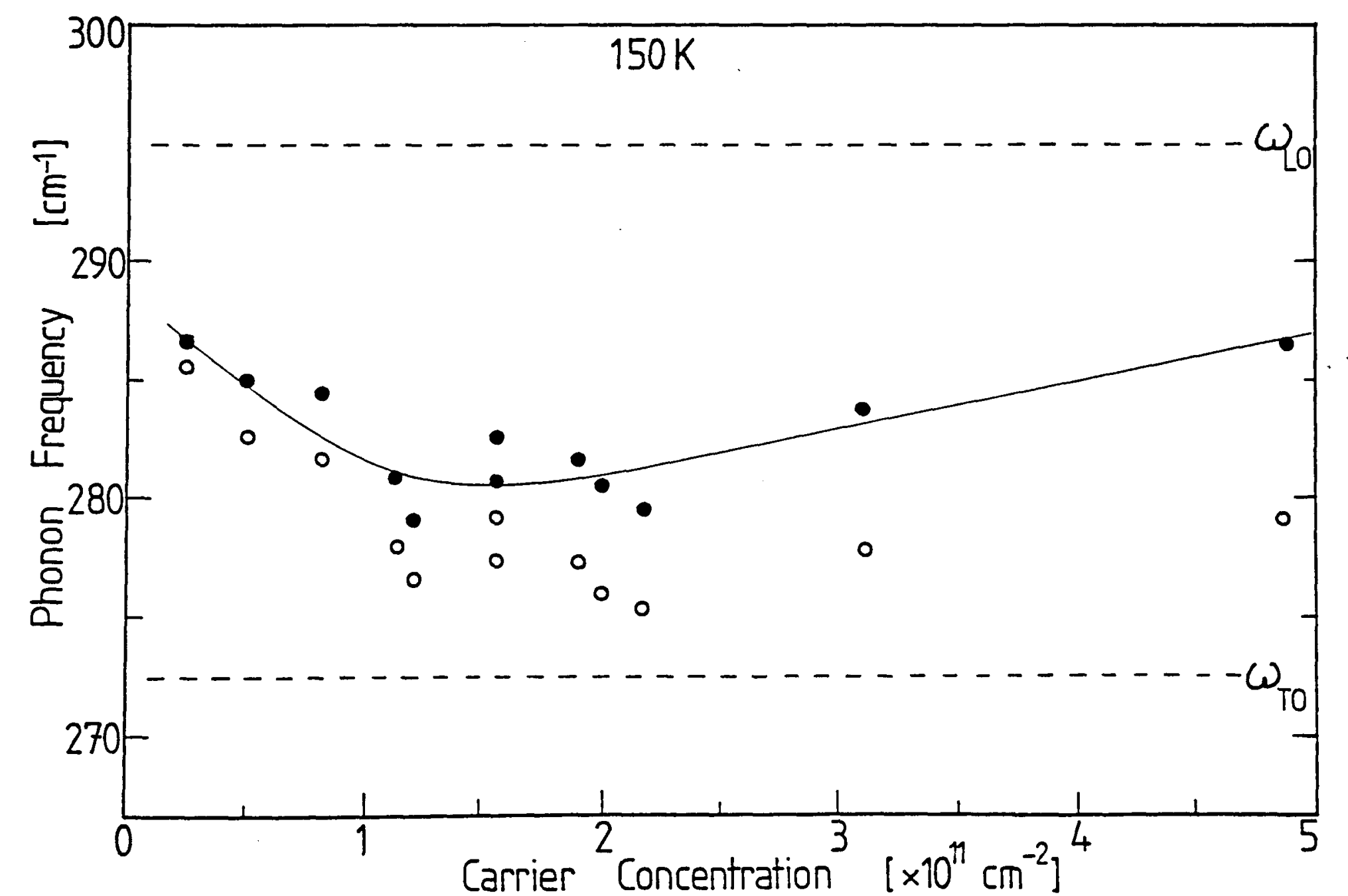

Figure 3.16 The data of Fig. 3.15 converted into apparent phonon frequencies using a suitable value of $\mathrm{m}^{*}{ }_{\mathrm{MPR}}$ for each sample, showing coupling well below the LO phonon frequency. Open circles show the effect of using Eqn. 3.18 to account for an exponentially damped resonance series. 


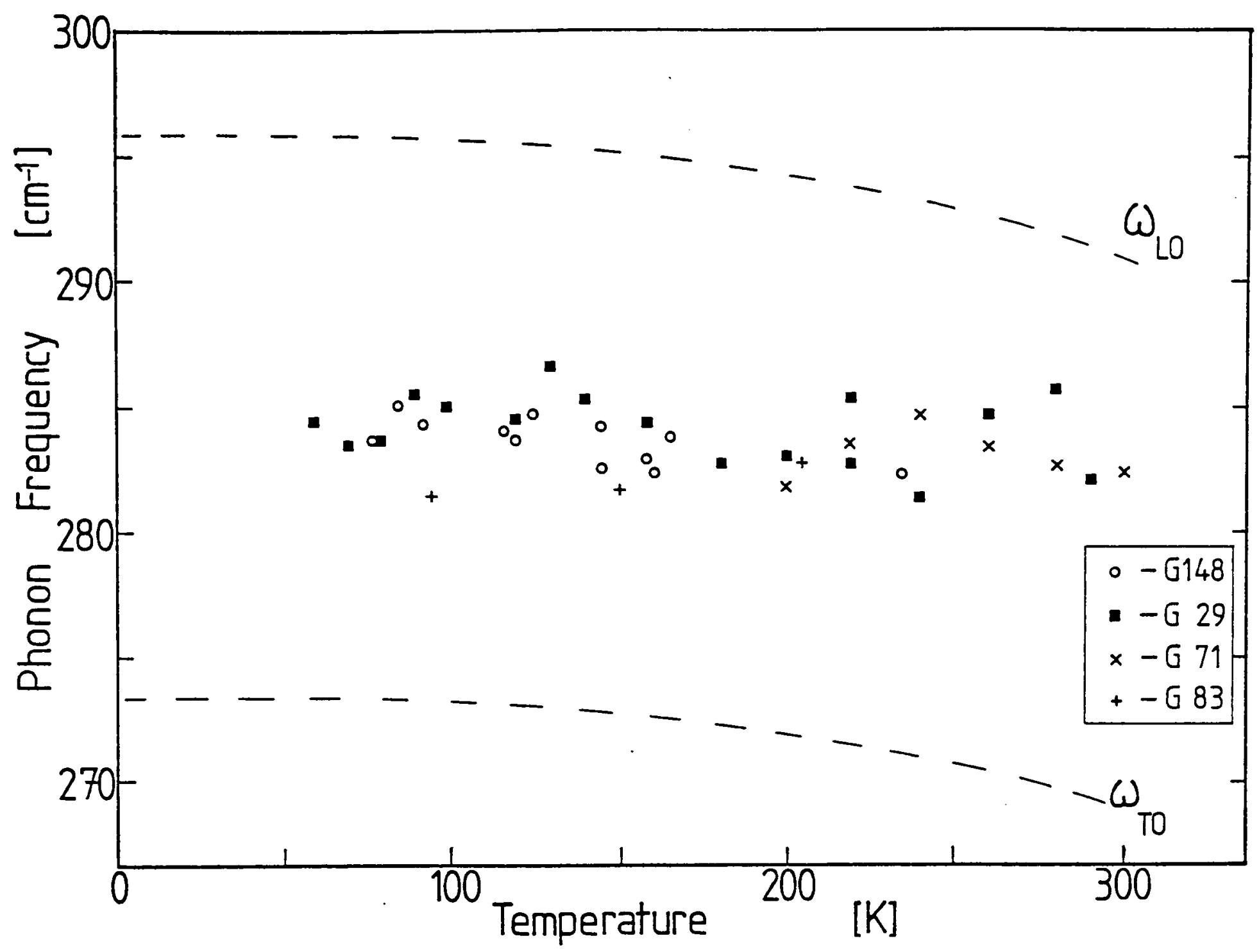

Figure 3.17 Position of the $N=3$ resonance for four $400 \AA$ spacer layer samples, showing good agreement between samples but little temperature variation.

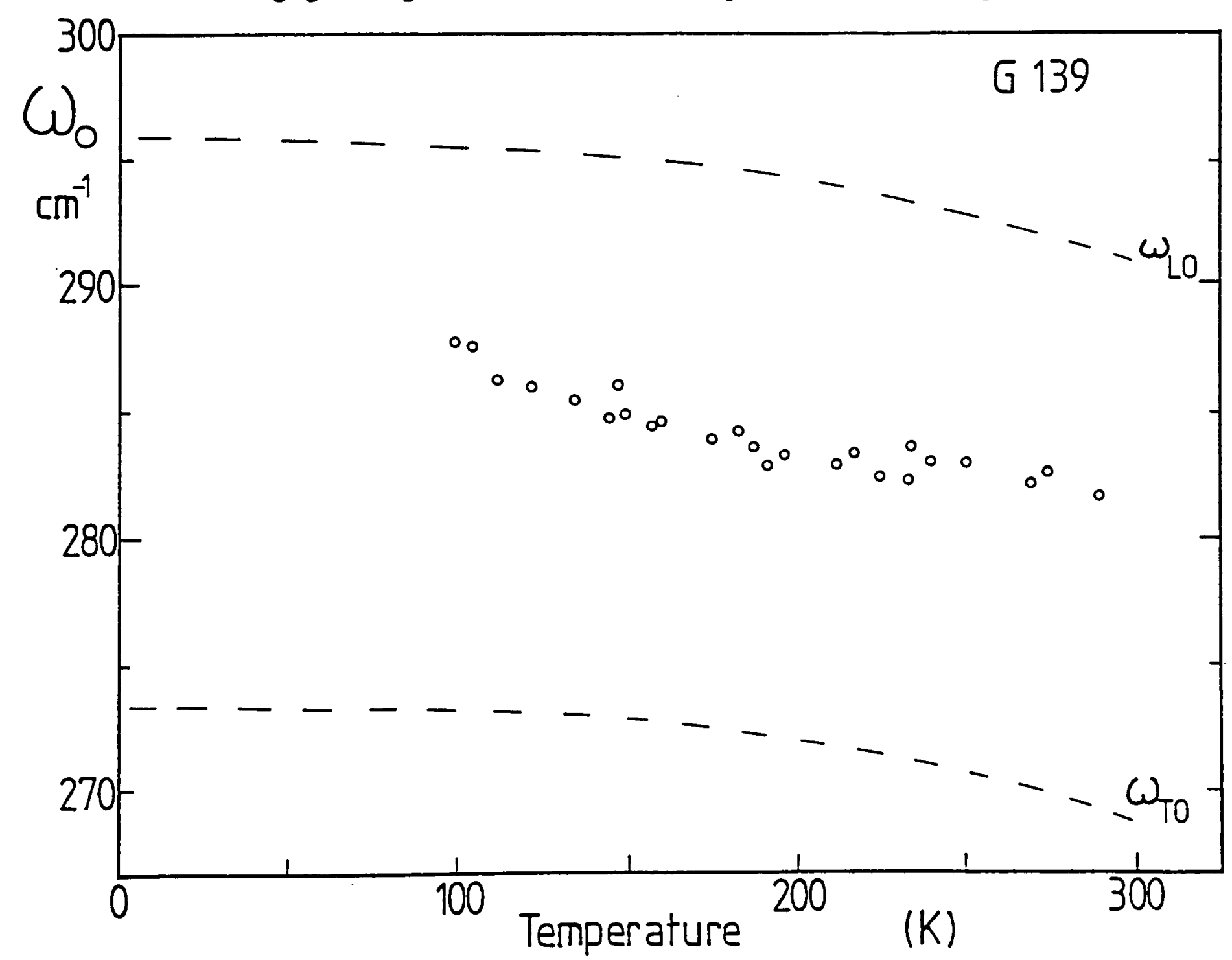

Figure 3.18 Position of the $\mathrm{N}=3$ resonance for G139, showing a similar temperature dependence to the phonon energies. 
that of the bulk LO mode at $295 \mathrm{~cm}^{-1}$; (ii) the resonance positions show a minimum at $\mathrm{n}_{\mathrm{s}} \sim 1.5 \times 10^{11} \mathrm{~cm}^{-2}$. It should be noted that the minimum is seen in both figures and so is not just an artifact of the particular mass values used, and that it occurs at a similar carrier concentration to the maximum in the resonance amplitude.

Figure 3.17 depicts the phonon frequency deduced from the resonance positions in a set of $400 \dot{A}$ spacer layer samples at temperatures between $77 \mathrm{~K}$ and $300 \mathrm{~K}$. There is very little variation of $\omega_{0}$ over this region and a remarkably good agreement between the samples. In Fig. 3.18 the same is shown for G139, with a similar amount of experimental scatter, but a definite increase in $\omega_{0}$ at lower temperatures. It is found that all samples of a particular spacer layer thickness (and hence similar $\mathrm{n}_{s}$ ) give the same phonon frequency and so in Fig. 3.19 lines have been drawn representing the average frequency for each family of samples, which emphasises the change of $\omega_{0}$ with carrier concentration (or $L_{s}$ ) found in Fig. 3.16.

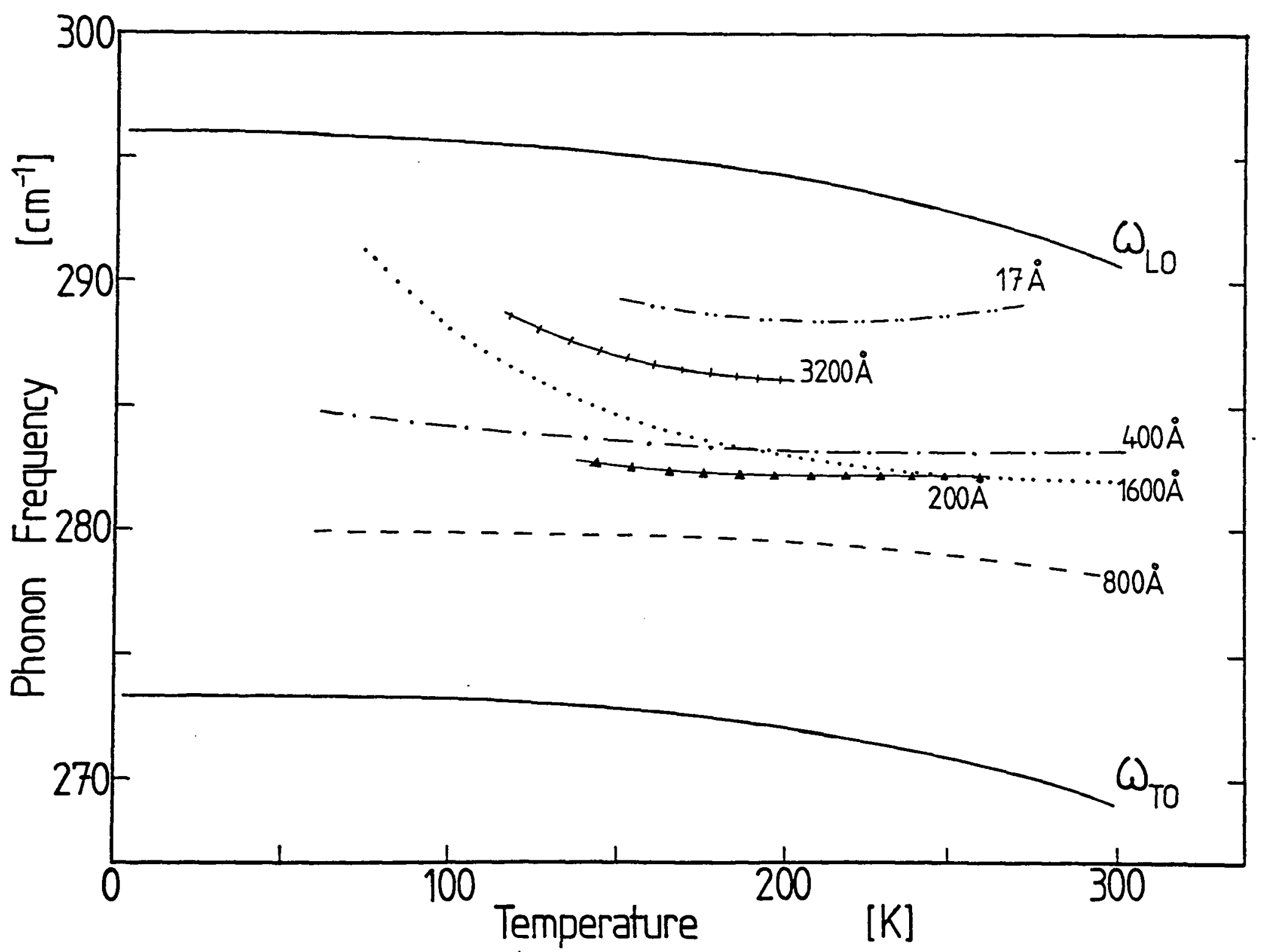

Figure 3.19 Position of the $\mathrm{N}=3$ resonance as a function of $\mathrm{T}$ for all the heterojunctions studied, shown by lines drawn through the average positions for samples of a particular spacer layer thickness. 


\subsubsection{Interpretation of the Positions}

The conclusion from these figures is that the phonon frequencies all lie in the range 279 to $289 \mathrm{~cm}^{-1}$, consistent with the earlier 'best estimate' of Brummell et al. (1987\&88) of $281 \mathrm{~cm}^{-1}$, and unexpectedly below that of the GaAs bulk LO phonon. There are a number of possible causes of this:

(i) The effective mass used in the calculations is, for some reason, too large.

(ii) Damping of the resonance may cause a shift in the resonance positions, which has not been accounted for.

(iii) The coupling really is to a phonon of lower energy than the bulk LO mode.

Let us briefly discuss the first two possibilities (the third will be discussed in Section 3.9 after results from quantum wells and the effect of rotating the magnetic field direction have been considered).

\section{(a) Reduction of the Effective Mass}

The effective mass would have to be reduced by $\sim 4 \%$ for the measured resonance positions to coporrespond to coupling to the bulk GaAs LO phonon. A number of corrections were applied to the effective mass used in the calculations, so let us first consider whether these could be varied. The correction for band non-parabolicity was chosen for the non-degenerate case, if degenerate statistics were required this would increase the mass still further.

There is more room for speculation over the resonant polaron correction, however as this is a resonant process it is extremely unlikely that this would be reduced by very much. The total down shift of phonon frequency due to this correction is $\sim 5 \mathrm{~cm}^{-1}$, so even if there was zero contribution from the resonant polaron coupling the phonon frequencies would still be too low. In order to fully account for the data the resonant polaron interaction would have to be modified so as to give a negative contribution to the effective mass, which seems unlikely.

A third consideration pertains to contributions from higher electric subbands which would act to reduce the effective mass. The mass difference between the subbands will 
be quite small and so would mean that nearly all of the activity would have to occur in the upper subband. The question of the subbands will be returned to in Section 3.9.

It may be that the mechanisms of cyclotron resonance and magnetophonon resonance are sufficiently different, as the different $\mathbf{k}$ of photons and phonons entails sampling different regions of the density of states, that comparison of the masses is not very meaningful. Wu et al. (1987) argue that cyclotron resonance positions can only be calculated by a dynamical conductivity approach and not in the effective mass approximation. However, both are resonant phenomena, involving local transitions, so would be expected to involve similar masses. Further, the use of cyclotron resonance masses to analyse 3-D magnetophonon experiments appears to work well, so we will continue to search for an alternative explaination.

\section{(b) Shift of the Resonance Positions}

The resonance positions have deliberately not been corrected for shifts due to damping. If the usual model with Lorentian line shapes is used the correction was shown to take $B_{F}$ to lower fields and hence produce even lower phonon energies, as illustrated by the open circles in Fig. 3.16.

A correction to higher fields is possible using a model for the density of states consisting of Gaussian broadened peaks (G) on a flat background (B), as favoured by Warmenbol (1989) and illustrated in Fig. 3.20. Contributions from each part of the density of states enters their calculation of the resistivity through the convolution of broadened initial and final states, and Fig. 3.20 shows that transitions including terms from the background give rise to the largest peak shifts (G-B). To account for the full discrepancy in phonon frequency a background of $30 \sim 50 \%$ of the zero field density of states would be required.

Peeters et al. (1989) have also investigated the decrease in phonon frequencies on increasing the temperature, which was reported in the early work of Brummell et al. (1987) and is seen in Fig. 3.18 for G139, but is not apparently a universal behaviour, since it is not demonstrated by the $400 \dot{A}$ and $800 \dot{A}$ spacer layer samples (Fig. 3.19). They find that this requires a temperature dependent broadening parameter 


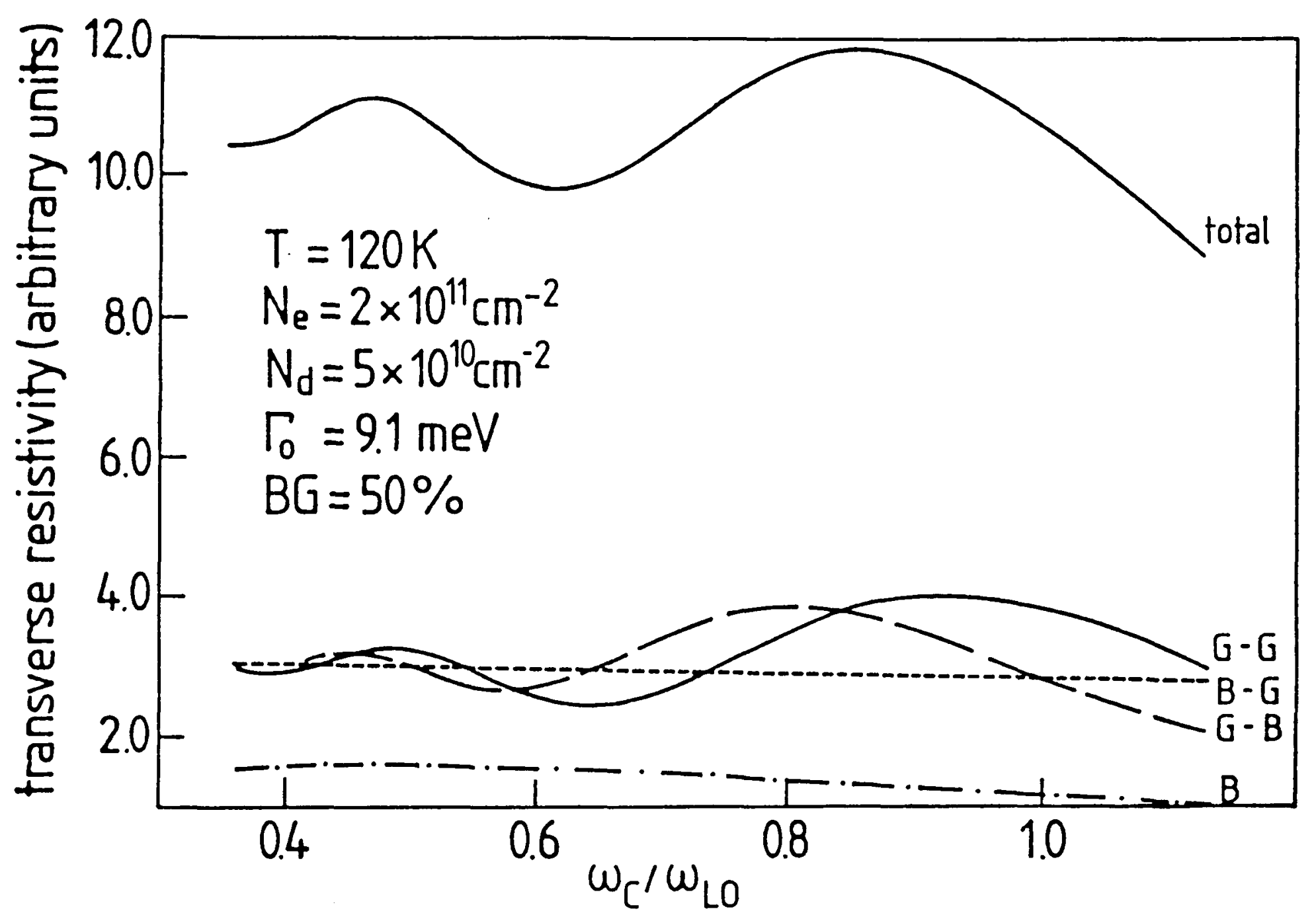

Figure 3.20 Magnetoresistance calculated by Warmenbol showing the contributions from the Gaussian (G) and background (B) parts of the density of states.

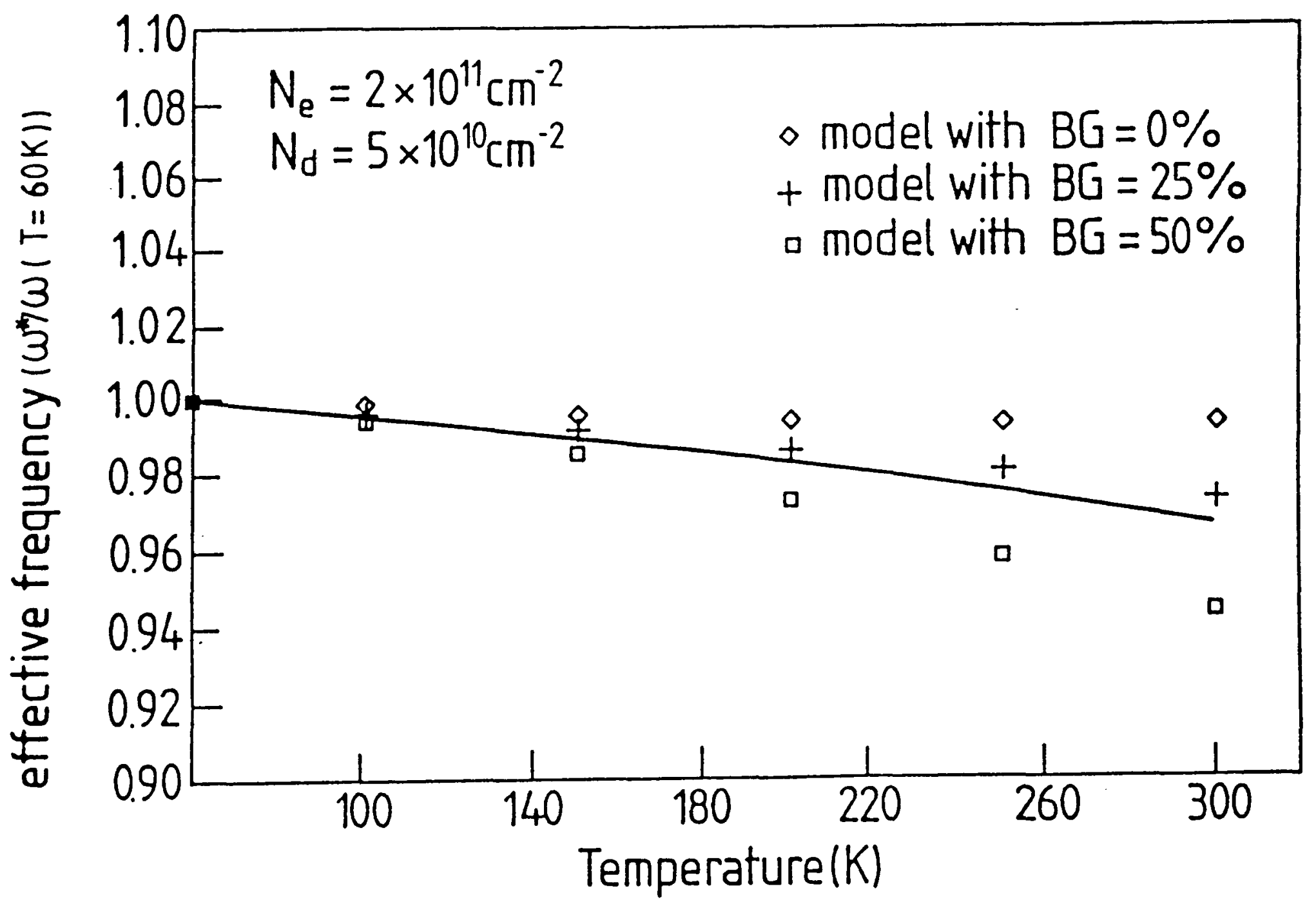

Figure 3.21 Calculated resonance position as a function of temperature for different \% background density of states. The solid line represents the experimental results of Brummell et al. (ie. is equivelent to the results presented here). 
$\Gamma(\mathrm{meV})=\left(\mathrm{T} / \mathrm{T}_{0}\right)^{1.7}$, with $\mathrm{T}_{\mathrm{o}}$ chosen as $60 \mathrm{~K}$. Their results for a variety of values for the background DOS are reproduced in Fig. 3.21 showing that any required decrease of $\omega_{0}$ with temperature could be modelled by an appropriate choice of background. However, it is not clear how this background density of states would affect the cyclotron resonance position. Peeters et al. dismiss the non-observence of a shift in cyclotron resonance by assuming these experiments are always performed at low temperatures (4.2K) where the Landau levels will be extremely sharp, but by measuring $\mathrm{m}^{*} \mathrm{CR}$ at the same temperature as the magnetophonon experiments are performed (Brummell et al. 1987) we may have already taken account of any shift in resonance position. Measurements of the $N=1$ resonance may demonstrate more clearly the direction in which the resonance positions have moved and so verify this model or otherwise.

The other major objection to this type of model is that if the damping was responsible for shifting the peak to lower fields, then it would be expected that the discrepancies would be largest where $\tilde{\gamma}$ was largest. $\tilde{\gamma}$ was seen earlier to increase monotonically with $n_{s}$, which would predict observation of the lowest phonon frequencies at the highest carrier concentrations and that at very low $n_{s}$ the shift down from the bulk LO phonon frequency should be negligable - clearly at varience with Fig. 3.16. However, it was found that increasing the density of states between Landau levels had a similar effect on the resonance position to broadening of the Gaussian peaks. So, to explain these results where the broadening of the peaks is known to increase with $n_{s}$, would require the background density of states to decrease as the carrier concentration increased. Physically this could be interpreted as a decrease in the random long range potential fluctuations in the sample when more electrons are present. 


\subsection{MAGNETOPHONON RESONANCES IN THE HALL COEFFICIENT}

Recordings are shown in Fig. 3.22, which show oscillations in both the resistivity $\rho_{\mathbf{x x}}$ and the Hall coefficient $\rho_{x y}$. The latter appear as minima at the positions of maxima in the original oscillations and are over an order of magnitude weaker. This may be understood by reference to the tensor nature of the resistivity:

$$
\rho_{x y}=\frac{\sigma_{x y}}{\sigma_{x x}^{2}+\sigma_{x y}^{2}}
$$

At high fields $\sigma_{x y} \gg \sigma_{x x}$ and so strong oscillations in $\sigma_{x x}$ will lead to weak oscillations in $\sigma_{x y}$ in antiphase to the original oscillations.

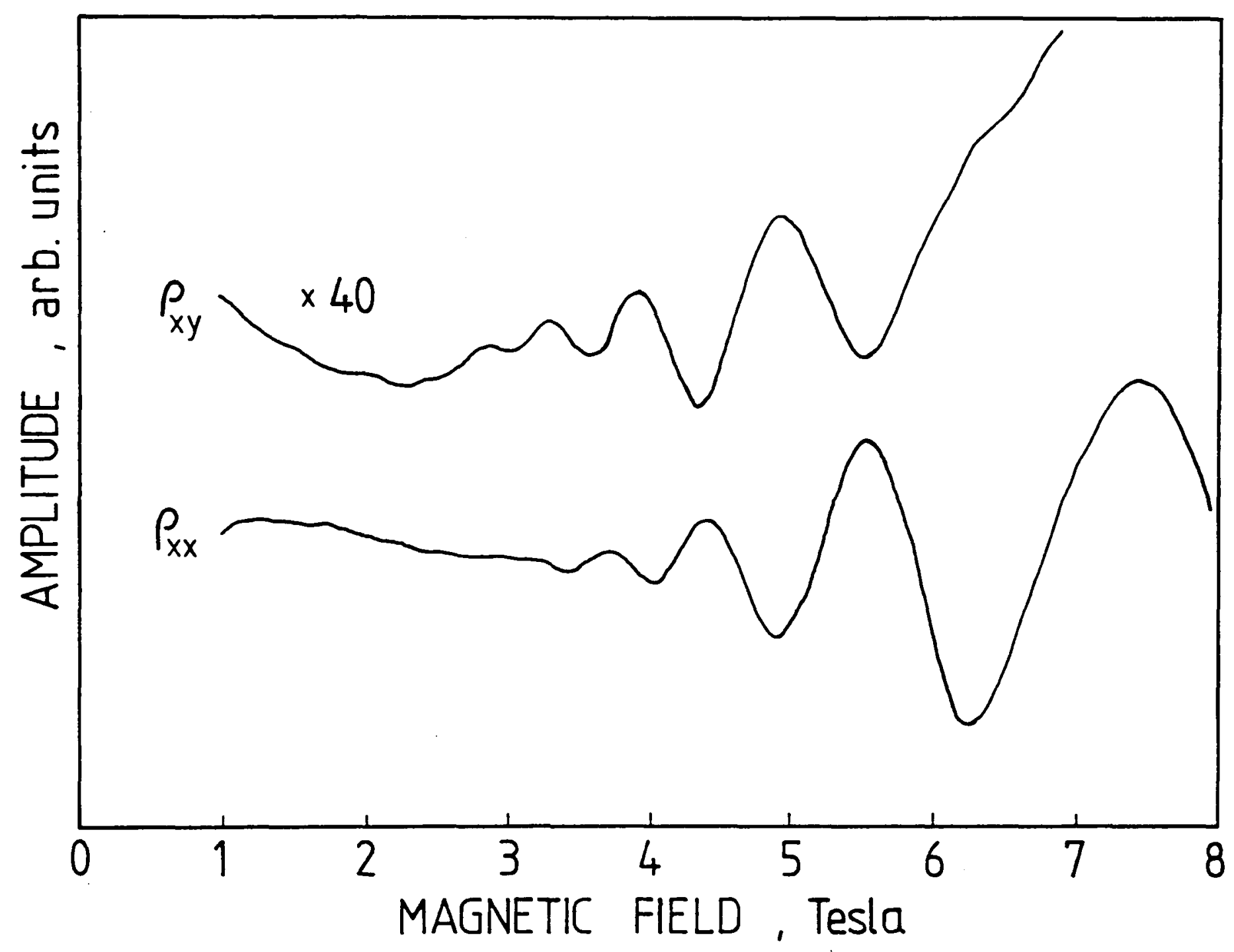

Figure 3.22 Second derivative recordings of $\rho_{x x}$ and $\rho_{x y}$ in $G 139$ at $150 \mathrm{~K}$, showing magnetophonon oscillations in the Hall coefficient a factor of $\sim 40$ weaker and inverted compared to the oscillations in $\rho_{\mathbf{x x}}$.

In 3D oscillations in the Hall coefficient have also been seen (Wood et al. 1970), appearing as minima in weakly polar materials such as InSb or InAs and as maxima in the more polar GaAs and InP. Maxima were predicted by Barker \& McSheehy (1973) to arise from oscillations in $\sigma_{x y}$, with the relative contributions of maxima or minima 
being determined by $\alpha$. However studies of InAsP by Nicholas et al. (1979) showed the peaks shifting position as the alloy composition was changed and showed little agreement with the theory. The fact that minima are observed here may mean that in $2 \mathrm{D} \sigma_{\mathrm{xy}}$ shows less oscillations than in bulk, or that the effective polaron coupling is weaker in GaAs heterojunctions than in 3D GaAs.

\subsection{ROTATION IN MAGNETIC FIELD}

\subsubsection{Effect on Resonance Amplitudes}

Tilting the magnetic field direction relative to the surface normal of the heterojunction through an angle $\theta$ usually has little effect upon magneto-transport phenomena, such as the quantum Hall effect and Shubnikov-deHaas oscillations, other than to demonstrate their two dimensionality. The features are found to depend only upon the perpendicular field component $B \cos \theta$. In contrast, something very different happens for magnetophonon resonances in a 2DEG. Figure 3.23 shows experimental recordings of the magnetophonon oscillations taken using the linear compensation technique, which demonstrate a very strong reduction in amplitude upon rotation. This is shown quantitatively for Samples G83 and G139 in Fig 3.24, where the amplitudes of the resonances are plotted as a function of angle. The amplitude decreases very rapidly, with rotation by approximately $10^{\circ}$ sufficient to halve it. This same behaviour was seen in all the heterojunctions studied and was in fact present in the early work of Tsui et al. (1980) and Kido et al. (1982), although they did not comment on it.

At higher angles there is apparently a subsidiary maximum in the amplitude, and even at $90^{\circ}$ there is still residual oscillatory structure. This is a consequence of some bulk conduction in the undoped, p-type, buffer layer of the heterojunctions caused by carbon impurities migrating from the substrate. The resistivity peaks observed at $\theta=90^{\circ}$ correspond to bulk magnetophonon resonance from light holes, with a fundamental field of 33T (Eaves et al. 1977). This causes some uncertainty in determining the exact resonance positions for the 2-D resonances at higher angles, and results in the pronounced 'wings' in the angular variation of the amplitude for lower carrier 
Figure 3.23 Compensated magnetoresistance recordings showing the effect of rotation, with $\theta$ as the angle between the sample normal and the magnetic field direction. Notice the shift in resonance position like $\cos \theta$ and the rapid fall in amplitude by $30^{\circ}$.

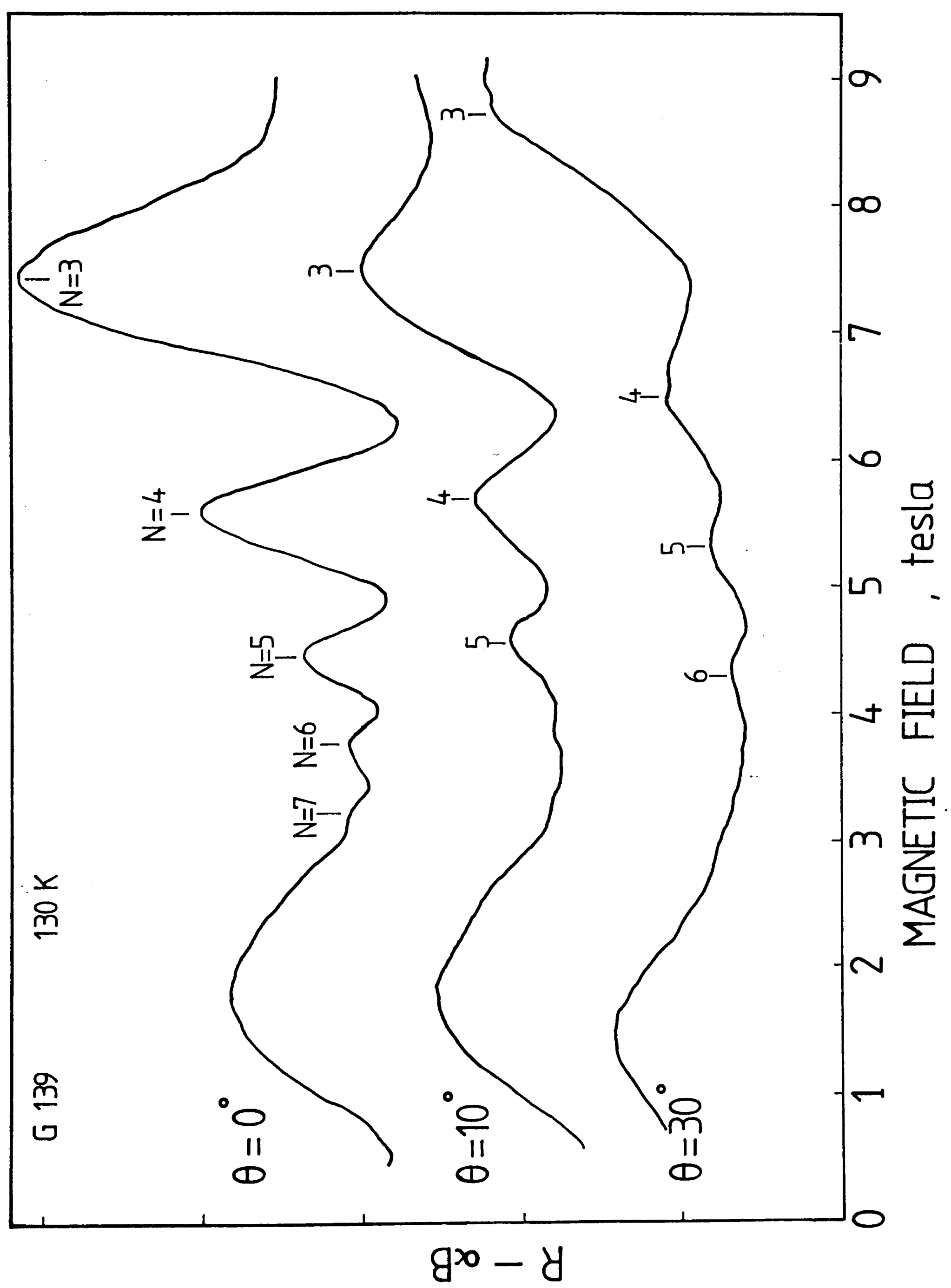




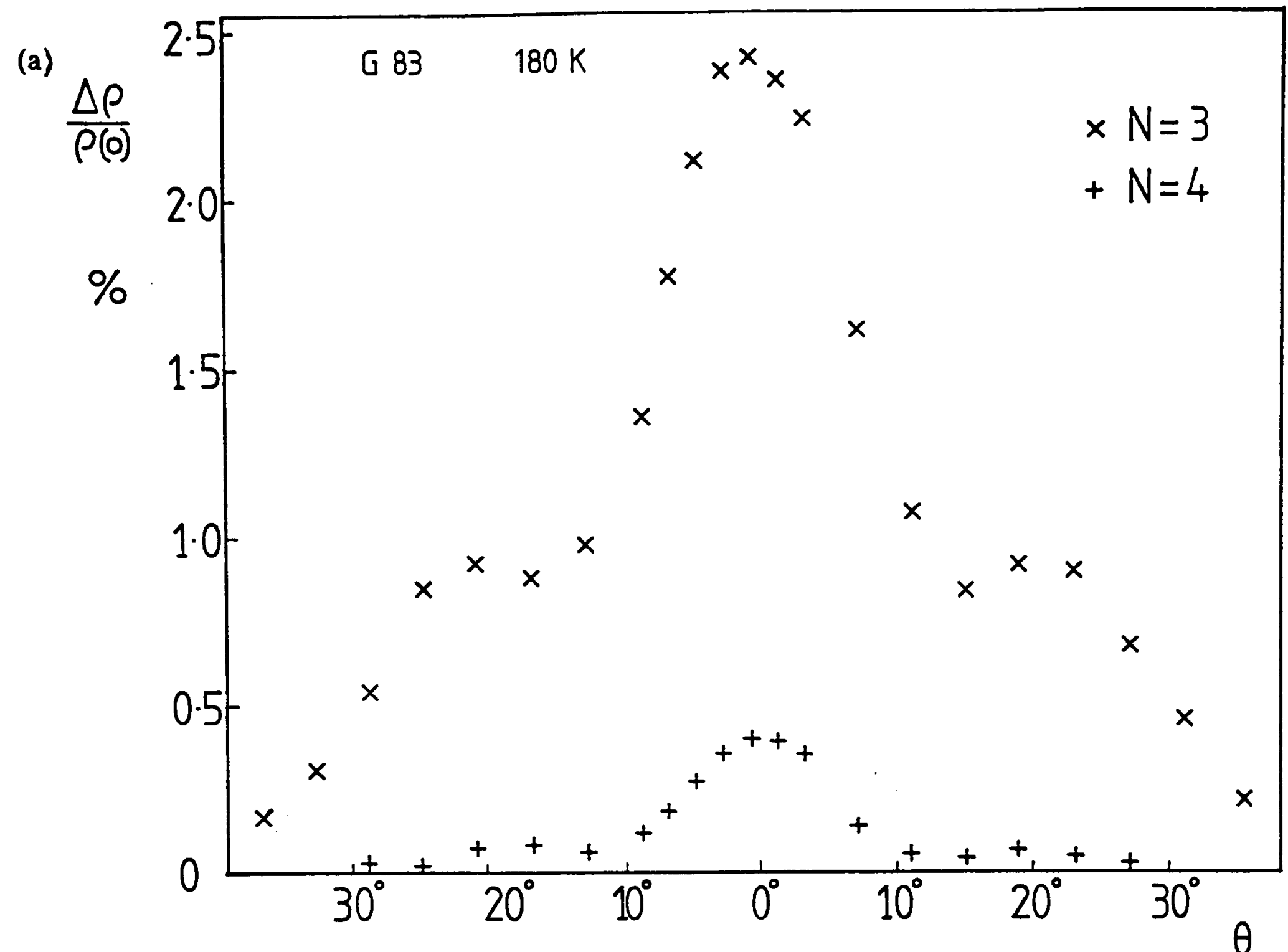

Figure 3.24 The rapid decrease in resonance amplitude on rotation observed in Fig. 3.23 shown quantitatively for G83 (above) and G139 (below). The structure in the wings is due to parasitic p-type resonances in the buffer.

(b)

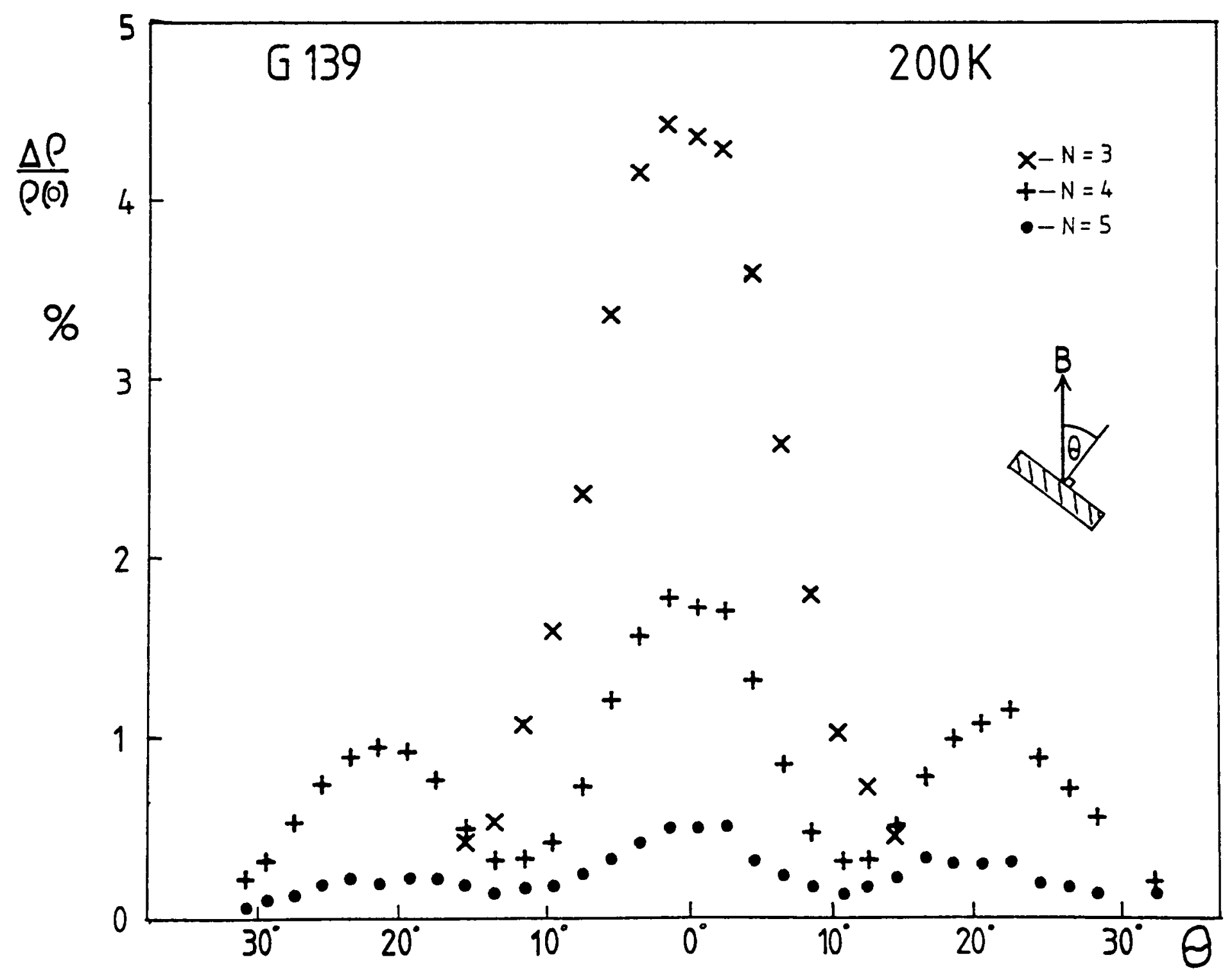


concentration samples (Fig. 3.24b).

The drastic reduction in amplitude was shown earlier (Fig. 3.7) for Sample G215, and enabled the 2D contribution to be separated from the bulk component which the same figure shows to be completely independent of sample orientation.

\subsubsection{Effect on Resonance Positions}

The resonance positions also show an anomalous behaviour, as illustrated in Fig. 3.25. This shows a dependence which initially follows $N B_{N}=B_{F} / \cos \theta$, as expected for a pure two-dimensional system, but then becomes more rapid, moving to a second $1 / \cos \theta$ curve with a resonance field $\approx 3 \%$ larger than would be expected from the $\theta=0$ value. The change in behaviour corresponds with the rapid decrease in amplitude. If the high angle results are used to deduce the phonon frequencies, we find the intriguing result that the frequency rises to almost that of the bulk L.O. phonon. This appears to

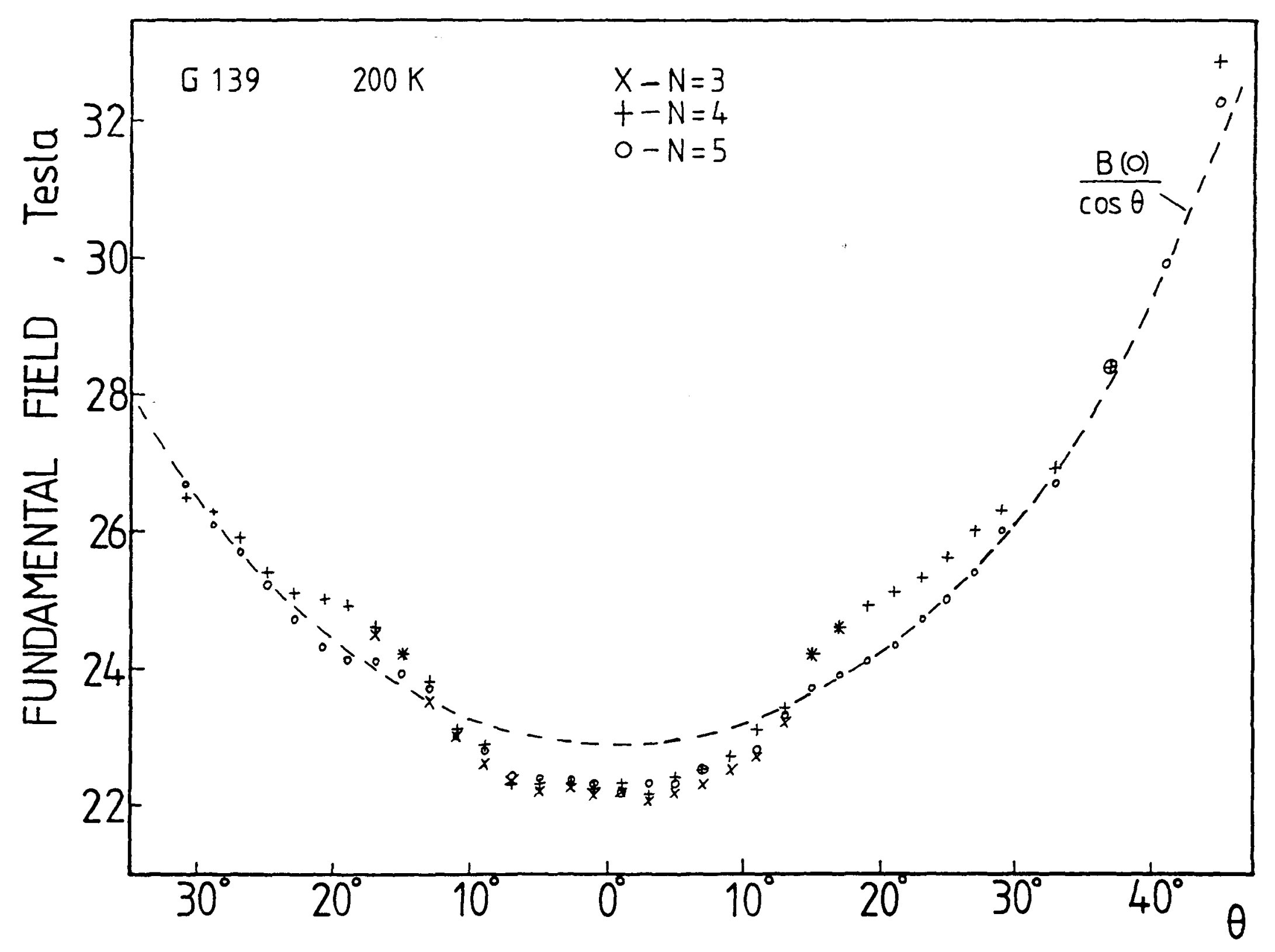

Figure 3.25 Variation in resonance position on rotation in a magnetic field, showing substantial deviations from the $\cos \theta$ law expected for pure $2 \mathrm{D}$ behaviour. 
be a general result for all of the samples studied, although the values in some cases are rather inaccurate due to the influence of the bulk resonances.

G215 shows a switch from 2D to n-type bulk behaviour at larger angles by the resonance position moving to a higher field, constant with rotation, from which a phonon frequency of $294 \mathrm{~cm}^{-1}$ would be calculated (using an appropriate $3 \mathrm{D}$ mass) compared with $276 \mathrm{~cm}^{-1}$ from the position at $0^{\circ}$ due to the $2 \mathrm{DEG}$. This provides good agreement with the bulk LO phonon as might be expected and emphasises that there is a real difference between the $2 \mathrm{D}$ and $3 \mathrm{D}$ resonances.

The reason for this anomalous behaviour on rotation is not clear, but is most probably associated with a mixing of the electric subbands of the heterojunction, which is the major consequence of introducing a magnetic field component along the direction of the interface (Ando et al. 1982). This will be discussed after considering magnetophonon resonance in structures which have a much larger difference in subband energies - quantum wells.

\subsection{RESONANCES IN GaAs-GaAlAs QUANTUM WELLS}

As remarked in Chapter 2 the growth of high quality GaAs-GaAlAs quantum wells is much more difficult than the corresponding single hetero-interfaces. Consequently the study of magnetophonon resonance in these systems has not produced as much data as for heterojunctions [although this is not so for GaInAs-InP discussed in Chapter 4 ].

There has been one solitary previous report by Mori et al. (1988a) where a complex series of oscillations were seen in the magnetoresistance of a $100 \dot{A}$ well with $\mathrm{n}_{\mathrm{s}}=1.7 \times 10^{12} \mathrm{~cm}^{-2}$, which is a suprisingly large carrier concentration at which to observe magnetophonon resonance. After Fourier transforming (a much over rated technique producing more than its share of dubious results) this revealed two series with reciprocal periodicities of $24 \mathrm{~T}$ and $45 \mathrm{~T}$. The lower of these represents interactions with ordinary LO phonons, but the 45T series was ascribed to interactions with LO phonons confined in the quantum well involving a transition between electric subbands. They also made some calculations for this process finding a periodicity of $41 \mathrm{~T}$. (Is 45 closer 
to 41 or twice 24 ?) It would have been intresting if they could have made comparison with other structures of different well width and hence different subband separations.

Five 30 period multiquantum wells, described in Chapter 2, have been studied. Problems were experienced in making electrical contacts to all the wells, as In does not diffuse easily through the GaAlAs barriers, resulting in a much higher noise level than in the heterojuctons. However, all showed a higher fundamental field of $23.7 \pm 0.2 \mathrm{~T}$ which corresponds to the bulk LO phonon frequency. The amplitudes of the resonances were much smaller than in heterojunctions and so were only observed in double differentiated traces. Assuming a similar response from the filters as for heterojunction resonances at the same magnetic field we find $\Delta \rho / \rho \sim 0.02 \%$ for the $N=4$ resonance in G176 with a carrier density of $\sim 1 \times 10^{11} \mathrm{~cm}^{-2}$ per well. This is to be compared with $\sim 1.5 \%$ in heterojuctions with similar $\mathrm{n}_{\mathrm{s}}$. Alternatively, if the total number of carriers in the multiple quantum wells were counted there would be similar amplitudes in both types of structure, however the damping factor was $\sim 1.4$ [calculated from second derivative traces and so somewhat inaccurate] which is similar to that in heterojunctions with $\mathrm{n}_{\mathrm{s}} \sim 1 \times 10^{11} \mathrm{~cm}^{-2}$, not $3 \times 10^{12} \mathrm{~cm}^{-2}$ and only slightly smaller than for heterojunctions with the same spacer layer thickness $(400 \dot{A})$. Thus it would appear that the first comparison is more useful, and that the magnetophonon resonance amplitude is much smaller in quantum wells than heterojunctions.

On rotation the resonance amplitudes remained constant and the fundamental field followed a straight $B_{d} / \cos \theta$ law, in contrast to the anomalous results found in the heterojunctions. This is depicted in Fig. 3.26 for Sample G176 which consists of thirty $100 \dot{A}$ wells with $\sim 1 \times 10^{11}$ electrons $/ \mathrm{cm}^{2}$ in each well. The carrier concentration per well was calculated from the low temperature Shubnikov-deHaas oscillation periodicity and the number of occupied wells found by comparing this value of $n_{s}$ with that deduced from the Hall coeficient (which depends on the total sheet carrier density).

Sample G177 had $1.2 \times 10^{11}$ electrons $/ \mathrm{cm}^{2}$ in thirty $300 \AA$ wells and showed resonances comparable in amplitude to those in a heterojunction of similar carrier concentration, 


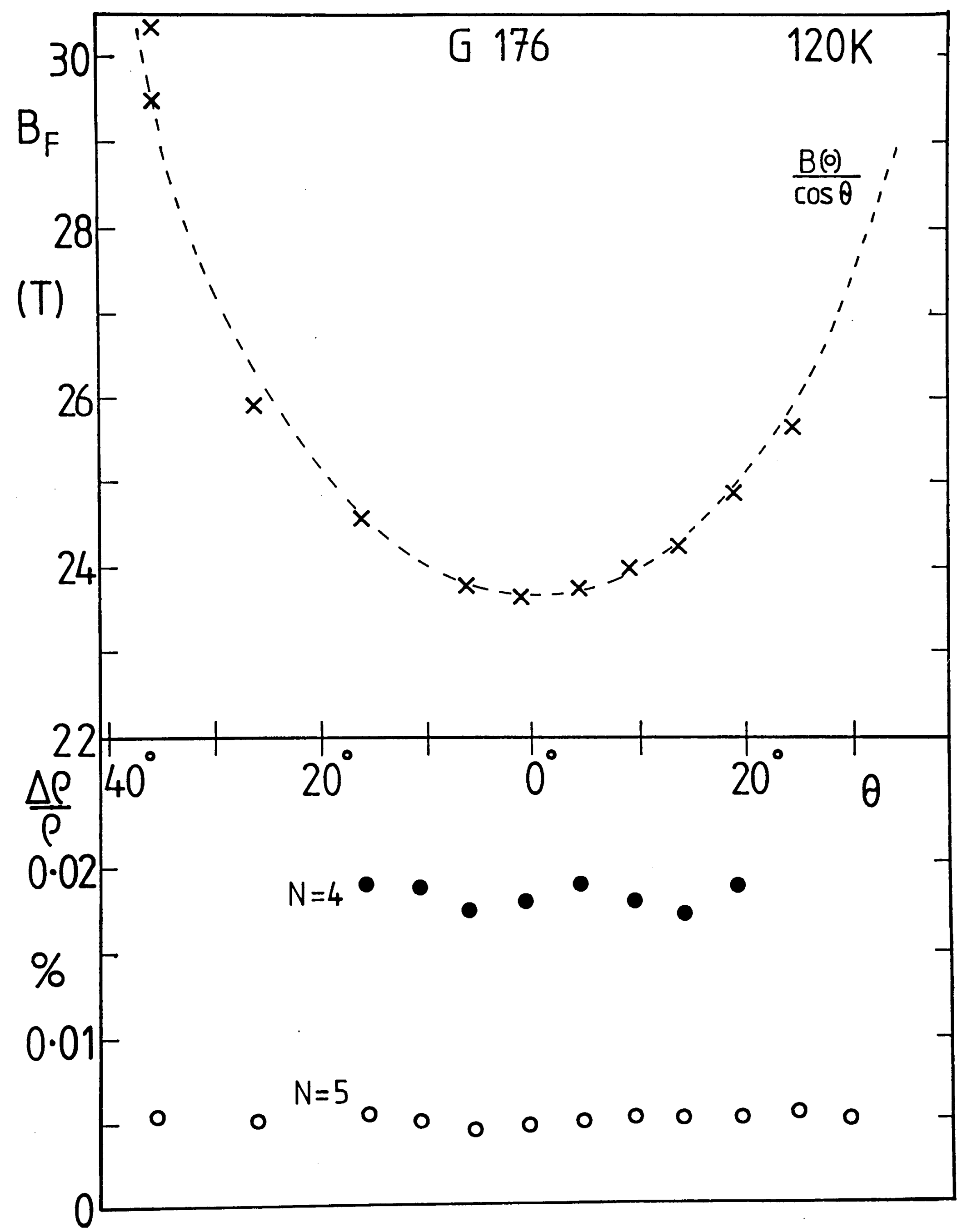

Figure 3.26 The effects of rotation in a magnetic field on the magnetophonon resonance from a GaAs-GaAlAs quantum well, showing substantial differences from the behaviour in heterojunctions: (i) the upper part shows a pure $\cos \theta$ dependence for the resonance position; (ii) the amplitude is much smaller and (iii) remains constant on rotation. 
and a similar decrease in amplitude at higher carrier concentrations. The fundamental field was higher than in heterojunctions at $23.7 \mathrm{~T}$, due to the larger mass in this sample. On rotation there were signs of the amplitude decreasing, but to a much smaller extent than in heterojunctions and the fundamental field did not vary to any detectable extent. Thus it appears that this sample with, $300 \AA$ wells, shows a behaviour somewhere between that of a heterojunction and a narrow quantum well, which is consistent with the extent of the wavefunction in heterojunctions being $\sim 300 \AA$.

These general differences in the behaviours of quantum wells and heterojunctions are also observed for GaInAs-InP (Chapter 5) and must be due to the differences in the shape of the potential: in heterojunctions the eigenstates have asymmetric wavefunctions, whereas those in quantum wells are symmetric. The other major difference is that the subband separation in quantum wells is fixed by the well width $(\sim 150 \mathrm{meV}$ in a $100 \dot{A}$ QW) and generally much larger than in heterojunctions, where values of $10 \sim 20 \mathrm{meV}$ are typical (see Chapter 6). This makes mixing of the subbands more of a possibility in low density heterojunctions than in low density quantum wells. At $180 \mathrm{~K}$ the thermal energy is $15.5 \mathrm{meV}$ meaning there will always be some upper subband occupation in the heterojunctions, whereas in narrow quantum wells this will only be the case for high carrier densities, such as the structure investigated by Mori et al. (1988a) where the Fermi energy is $760 \mathrm{meV}$ and so gives more chance of upper subband occupation. The $300 \AA$ wells of G177 have a subband separation of only $\sim 18 \mathrm{meV}$ and so it is not suprising that this structure looked more similar to the heterojunctions than did G176.

\subsection{SOME PLAUSIBLE EXPLAINATIONS ?}

A coherent picture of these anomalous results now begins to emerge. In heterojunctions, in perpendicular field, the phonon energies deduced from the resonance positions are well below that of the bulk LO phonon, with the lowest phonon energies being deduced in cases where the resonance amplitudes are the largest. On rotation of the magnetic field, the amplitude is found to decrease and the resonance position moves up in field. The quantum wells also fit in this picture, with a small amplitude, 
constant on rotation, and a coupling at the bulk LO phonon frequency. Thus it appears that the 2D magnetophonon effect may be explained by finding some strong coupling below the LO frequency, which can only be utilised under certain conditions. These conditions would appear to be related to the subband structure of the system and be breached when the subbands are mixed or are too far separated in energy.

\subsubsection{Possible Phonon Modes}

In Section 3.5 it was suggested that there could be a coupling to a phonon mode with an energy below the bulk LO mode. The major piece of evidence against this idea comes from the cyclotron resonance measurements of Langerak et al. (1988) on similar GaAs-GaAlAs heterojunctions at low temperatures, where only coupling to the bulk LO phonons is seen. If there were to be any screening or occupation effects at higher temperatures they would be expected to be stronger at $4 \mathrm{~K}$ and so should be observed in these experiments. The processes of optical transmission and electrical transport are however different so we will press on and investigate the possible candidates for the mystery phonon:

(a) Strong screening of the polarization field of the LO phonons would lead to a reduction in their frequency. That this would be so can be seen by considering that the frequency difference between an LO and TO phonon is due to the bulk E-field of the LO mode (Lassnig 1986). However this seems rather unlikely because the screening should be strongly temperature and electron concentration dependent, due to changes in both the level broadening and the carrier statistics.

(b) In reflectivity studies $\mathrm{Kim} \&$ Spitzer 1979 found that $\mathrm{Ga}_{1-\mathrm{x}} \mathrm{Al}_{\mathrm{x}} \mathrm{As}$ exhibits two mode behaviour, with 'GaAs-like' LO and TO frequencies of 282 and $269 \mathrm{~cm}^{-1}$ and 'AlAs-like' frequencies of 379 and $359 \mathrm{~cm}^{-1}$ (when $\mathrm{x}=0.3$ as in most of these samples). The measured phonon frequencies thus correspond well with the 'GaAs' LO mode of GaAlAs, and interactions with this phonon have been observed in the Raman measurements of Maciel et al. (1987), although scattering by the confined modes in the GaAs was stronger. It seems unlikely that interactions across the barrier should dominate over coupling to the bulk LO mode, or that the $\sim 1 \%$ 
wavefunction penetration of the barrier should determine the couplings.

(c) Coupling could be to interface phonon modes. These are phonons which are localised within the region where the lattice notices the effect of the interface. In a GaAs-GaAlAs heterojunction there will be modes at 290 and $270 \mathrm{~cm}^{-1}$, but with weaker oscillator strength than the bulk LO mode.

(d) An alternative to interface modes is the possibility of continuous modes propagating across the interface and that the measured frequency is near the top of the region where LO phonon bands of the two materials overlap. An example of this is in GaInAs-AlInAs heterojunctions where Brummell et al. (1983) found the magnetophonon was dominated by the InAs LO mode which could propagate in both alloys, by contrast the GaAs mode predominated in GaInAs-InP [but see Chap. 4].

(e) Another explaination could involve unresolved coupling to two modes close in energy, such as the bulk GaAs LO phonon at $296 \mathrm{~cm}^{-1}$ and TO phonon at $273 \mathrm{~cm}^{-1}$. However, it is clear that interaction with some mode lower in frequency than the bulk GaAs LO would be needed to explain the results.

(f) This unresolved combination may be some form of coupled plasmon-phonon mode.

Even if any of these possible couplings do exist, there remains the question of why they are able to dominate over coupling to the bulk LO mode.

\subsubsection{Interface Phonons}

Coupling to interface modes will decrease exponentially with the distance from the interface. The extent of the region of coupling is inversely proportional to the phonon wavevector in the plane of the interface. [The coupling strength decays like $\exp \left(-\mathrm{k}_{\perp} z\right)$.] This length will be of the order of the cyclotron radius, and may be comperable to the extent of the wavefunction in the $z$-direction $(\sim 300 \dot{A})$, but it is still not clear that these modes would dominate over the bulk LO phonons. The interaction with interface modes would be expected to increase as the electron wavefuction is compressed towards the interface, which occurs at higher electron densities - giving the required electron density dependence for $\Delta \rho / \rho$.

The energies of the interface modes at a single interface are given by the condition 
that the real part of the dielectric constant $\left(\epsilon_{1}+\epsilon_{2}\right)$ should equal zero. The dielectic constant for the alloy can be written as:

$\epsilon=\epsilon_{\infty}+\left(\epsilon^{\prime}-\epsilon_{\infty}\right) \frac{\omega_{T}^{2}\left(\omega_{T}^{2}-\omega^{2}\right)}{\left(\omega_{T}^{2}-\omega^{2}\right)^{2}+\gamma^{2} \omega^{2}}+\left(\epsilon_{0}-\epsilon^{\prime}\right) \frac{\omega_{T}^{2},\left(\omega_{T}^{2},-\omega^{2}\right)}{\left(\omega_{T}^{2},-\omega^{2}\right)^{2}+\gamma^{2} \omega^{2}}$

where $\omega_{\mathrm{T}}$ and $\omega_{\mathrm{T}}$, are the frequencies of the two TO phonons and $\epsilon_{0}$ and $\epsilon_{\infty}$ are the low and high frequency dielectric constants, and $\epsilon^{\prime}$ is defined by the generalised Lyddane-Sachs-Teller relations

$$
\frac{\epsilon^{\prime}}{\epsilon_{\infty}}=\frac{\omega_{L}^{2}}{\omega_{T}^{2}} ; \quad \frac{\epsilon_{0}}{\epsilon^{\prime}}=\frac{\omega_{L}^{2}}{\omega_{T}^{2}}
$$

For the GaAs there is only one phonon branch and Eqn. 3.21 can be used with $\epsilon^{\prime}=\epsilon_{\infty}$. The result is interface modes with frequencies of 290 and $270 \mathrm{~cm}^{-1}$.

Calculations of the interaction strengths of this type of phonon have been performed for quantum wells or superlattices (Lassnig \& Zawadzki 1984a; Sawaki 1986; Wendler \& Haupt 1987) but there have been no reports for single interfaces, where the $\mathbf{k}$-vector is not well defined and the calculations are more complex. Taking a Fang-Howard wavefunction suitable for a triangular well as $\Psi \propto \mathrm{zb}^{3 / 2} \exp (-\mathrm{zb})$ and the electric field due to the interface mode as $E_{\text {int }}=E_{0} \exp \left(-k_{\perp} z\right)$ the coupling matrix element is:

$$
\begin{aligned}
\left\langle\Psi^{*}|E| \Psi\right\rangle & \propto \int_{0}^{\infty} z^{2} b^{3} e^{-\left(2 b+k_{\perp}\right) z} d z \\
& \propto \frac{b^{3}}{\left(2 b+k_{\perp}\right)^{3}}
\end{aligned}
$$

Since $b \sim n_{s}^{1 / 3}$ and $k_{\perp}$ is carrier concentration independent, the coupling strength shows the required increases with $n_{s}$, provided $k_{\perp}>b$.

In a quantum well the wavefunction for an electron in the lowest subband is symmeteric about the centre of the well and so coupling to interface modes, with electric fields perpendicular to the interfaces, would be expected to be much weaker than in heterojunctions.

It would appear that a number of the experimental observations could be explained by coupling to interface modes, but it is not at all clear why this should be preferable 
to coupling to bulk LO phonons. Also there is no obvious way of explaining the disappearance of the resonances on rotation of only a few degrees, unless the mixing of the subbands radically alters $k_{\perp}$ (which will then not be a good quantum number) or the screening in the $z$-direction.

\subsubsection{Subband Stuctures}

The differences between quantum wells and heterojunctions together with the dramatic reduction in amplitude and shift in frequency of the oscillations on rotation of the latter suggest that the key to understanding the $2 \mathrm{D}$ resonances lies with the influences of the subband structure on the electron-phonon coupling.

One possible mechanism for this influence could be a modification of the screening of charge fluctuations in the $z$-direction. In a perfectly quantised 2-D system the subband wavefunction is unable to distort in order to provide screening of fields in this direction, whereas fields in the plane of the 2DEG can be very strongly screened.

Let us consider the mechanism for a magnetophonon resonance transition. In order for an electron to make the transition between Landau levels it must change its wavevector, $\mathbf{k}$, in the plane of the 2DEG. Thus the only interactions will be with phonons having $\mathbf{k}$ parallel to the hetero-interface. For 3D LO phonons if $\mathbf{k}$ is parallel to the interface then the electric field must lie in the plane of the 2DEG and hence may be very strongly screened in high magnetic fields. If it were possible to couple to a mode with an electric field perpendicular to the interface this would not be screened and so there could be stronger coupling. Such a mode could be a transverse optic phonon. The mixing of the subbands on rotation would then allow a large increase in this 'perpendicular' screening and so drastically reduce coupling to this mode, thus allowing the expected LO phonon scattering to be observed.

However, there are certain objections to this picture: (i) Screening effects should be stronger at higher carrier concentrations, but the same behaviour is seen for all samples. (ii) Screening should have little effect at high temperatures and should be stronger at larger magnetic fields, yet the same behaviour is seen for amplitudes of all harmonic numbers studied and there is no apparant temperature dependence of the resonance 
position. (iii) The observed amplitudes correspond well with results on bulk GaAs, both in magnitude and temperature dependence, so why do we now say LO phonon coupling is very weak and try invoking strong couplings to other modes?

The third objection can be over ruled by reference to the discussion of resonance amplitudes. Since this is a resonant process the phonon coupling just establishes a discontinuity in the magnetoresistance. The amplitude of the resonance would be infinite but for the other factors acting to broaden it, which will be just the same whatever mode is being coupled to.

\subsubsection{Coupling to TO Phonons?}

One possible phonon mode with a frequency below that of the LO phonon which could couple with the electrons, and would change its coupling as the subband structure is altered, is the TO phonon, as suggested by Brummell et al. (1987). There remains the unresolved question of the origin of coupling to the TO mode, and its dominance over LO phonon absorption. A possible mechanism for the appearance of scattering by the TO phonons would arrise from their finite size, due to a finite lifetime, which means that they possess a finite surface electric field which can couple to the electrons via the deformation potential.

The phonon 'size' may be estimated from the energy uncertainty, and hence the k-vector spread which could be calculated from the phonon dispersion curve measured by Raman or neutron scattering. A lifetime of $\sim 7 \mathrm{ps}$ at $77 \mathrm{~K}$ was found using a time resolved Raman measurement (von der Linde et al. 1980), which corresponds to a size of $\sim 30 \dot{A}$. Calculation of the resulting surface electric fields suggests values at least an order of magnitude lower than for the LO phonon. However, one polarisation of TO phonon will have an electric field perpendicular to the interface, which is unscreened, and may also have an additional interaction through its influence on the subband levels. Thus the TO mode may give significant scattering until the subbands are mixed, after which its intensity falls and the strongly screened LO coupling dominates over the weakly screened TO coupling.

By contrast, in quantum wells the subband separation is large and so there would be 
a much smaller coupling to the TO modes. This would leave only screened interaction with the LO modes, which we see as a small resonance amplitude, and no change in resonance amplitude, or position, on rotation.

There is only fairly weak experimental support for this conjecture based on studies of resonant polaron coupling in GaInAs based heterojunctions (Nicholas et al. 1985) and HgCdTe accumulation layers (Singleton et al. 1986a; Nicholas \& Sigg), where coupling close to the TO frequencies has been suggested. However, better analysis of the results on GaInAs (Huant et al. 1989) reveal that the supposed coupling was at least part due to the dielectric response function. The data on $\mathrm{HgCdTe}$ is always complicated by the large numbers of occupied subbands etc. Contrary evidence comes from the high field cyclotron resonance work of Langerak et al. (1988), where no coupling was seen at the TO frequency. It must be remembered that the two resonance processes are very different and so observation, or otherwise, of an effect in one experiment does not necessarily have implications for the other. Thus it appears that the best evidence for TO coupling is actually in these magnetophonon resonance results.

\subsubsection{Coupled Plasmon-Phonon Modes}

In polar semiconductors the LO phonons may couple to plasma excitations of the electron gas and the coupling to $3 \mathrm{D}$-plasmons has been extensively studied by Raman scattering on bulk GaAs. The motivation for considering this is that the mixed plasmon-phonon modes may have a frequency smaller than the LO phonon and so be able to explain the anomalous magnetophonon resonance positions.

The different collective excitation modes are determined from the zeros of the dielectric function which has been calculated by $\mathrm{Wu}$ et al. (1985) as:

$$
\epsilon(\omega)=\epsilon_{\infty} \frac{\left(\omega^{2}-\omega_{\mathrm{LO}}^{2}\right)}{\left(\omega^{2}-\omega_{\mathrm{TO}}^{2}\right)}-\frac{2 \pi \mathrm{e}^{2}}{\mathrm{k}} \chi(\mathrm{k}, \omega)
$$

where $\epsilon_{\infty}$ is the high frequency dielectric constant and $\chi(k, \omega)$ is the polarization of the electron gas which is evaluated within the random phase approximation. The first term of Eqn. 3.25 represents the background and the second term is the contribution to the 
dielectric from the electron gas. In the long wavelength limit $k \ll k_{F}$ there are three modes: one close to the LO phonon frequency and two plasmon-like modes with $\omega \propto \mathrm{k}$. None of these have frequencies between the LO and TO phonons and so are of no interest. However if $\mathrm{k}$ is sufficiently large $\left(\gg \mathrm{k}_{\mathrm{F}}\right)$ there is only one mode:

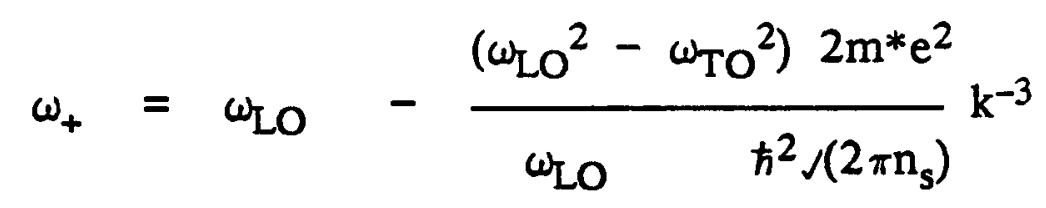

which approaches $\omega_{\text {LO }}$ asymptotically from below. The calculations of Wu et al. show further that this short wavelength limit is reached at lower $\mathrm{k}$ when the electron density is larger. In transport measurements $\mathrm{k} \approx \mathrm{k}_{\mathrm{F}}$ and the calculations suggest that to see a mode between the LO and TO phonons requires $n_{s} \geqslant 3 \times 10^{11} \mathrm{~cm}^{-2}$.

This frequency range is however allowed with small wavevector for coupled intersubband-plasmon-LO-phonon modes provided the subband slitting, $E_{01}=\hbar \omega_{01}$, is above the TO phonon energy. The attraction of involving intersubband excitations should be apparent from the discussion of Section 3.10.3. These modes have been calculated by Yuasa \& Ishii (1988), modifying Eqn. 3.25 by subtracting a term $\epsilon_{\infty} \omega_{p}^{*} /\left(\omega^{2}-\omega_{01}^{2}\right)$ where:

$$
\omega_{\mathrm{p}}^{*} \quad=\frac{2 \mathrm{n}_{\mathrm{s}} \omega_{01}}{\hbar} \mathrm{V}_{01}
$$

is the effective plasma frequency and $V_{01}$ is the Coulomb interaction for the ground and first excited subbands. The coupled modes are then given by:

$\omega_{ \pm}^{2}=\frac{1}{2}\left\{\omega_{01}^{2}+\omega_{L O}^{2}+\omega_{p}^{* 2}\right\}+\frac{1}{2}\left[\left\{\omega_{01}^{2}+\omega_{L O}^{2}+\omega_{p}^{* 2}\right\}^{2}-4\left\{\omega_{01}{ }^{2} \omega_{L O}{ }^{2}+\omega_{T O}^{2} \omega_{p}^{* 2}\right\}\right]^{\frac{1}{2}}$

Yuasa \& Ishii (1988) also reported Raman measurements in modulation doped multi-quantum wells in which the $\omega_{-}$mode was seen at frequencies between $270 \mathrm{~cm}^{-1}$ and $279 \mathrm{~cm}^{-1}$, which is in the same region as the magnetophonon coupling. But, although this mode was seen provided $n_{s} \geqslant 3 \times 10^{11} \mathrm{~cm}^{-2}$, it did not appear stronger than the LO mode until $\mathrm{n}_{\mathrm{s}} \sim 10^{12} \mathrm{~cm}^{-2}$ - ie. many more carriers than present in our samples. Further all the results are from quantum wells not heterojunctions, where it is found (in Chapter 6) that $\omega_{01} \ll \omega_{T O}$ again until $\mathrm{n}_{\mathrm{s}} \sim 10^{12} \mathrm{~cm}^{-2}$. Thus it would appear that if coupled modes are to be invoked it must be at large $k$ values. 


\section{$\underline{3.10 \quad \text { SUMMARY }}$}

Magnetophonon resonance has been studied in GaAs-GaAlAs heterojunctions as a function of temperature, electron concentration and magnetic field orientation. The damping factor is found to increase with carrier concentration and is always greater than found in 3D. This variation is found to be consistent with remote impurity scattering determining the broadening of the levels. The amplitude shows a systematic variation with carrier concentration, with a maximum appearing for $\mathrm{n}_{\mathrm{s}} \sim 2 \times 10^{11} \mathrm{~cm}^{-2}$ : there is a rapid decrease at high $n_{s}$, explained by the increase in damping factor; and the increase with $n_{s}$ at lower carrier concentrations is attributed to occupation effects.

The resonance positions give phonon frequencies well below the bulk LO value when the field is perpendicular to the heterojunction interface, with the minimum phonon frequencies being found under the same conditions as the maximum resonance amplitudes. This suggests that there is some additional coupling at a frequency below that of the bulk LO phonon, which can be suppressed under certain conditions to leave a weaker coupling at the LO frequency. On applying a magnetic field component parallel to the $2 \mathrm{DEG}$ the resonance amplitudes decrease rapidly in heterojunction samples, but not in quantum wells. This decrease in intensity is accompanied by the phonon frequencies moving up towards the bulk LO value. In quantum wells magnetophonon resonance is also observed, but the amplitudes are much weaker and the phonon frequencies deduced agree well with the bulk LO phonon. These differences are linked to the subband structure of heterojunctions and quantum wells. Thus effects in heterojunctions may be due to anisotropic screening and its modification by subband mixing. Alternative phonons for coupling have been investigated, but no really suitable candidates are found! The best bet appears to require coupling to interface modes. Otherwise an unresolved combination of LO and TO modes or coupled plasmon-phonon modes are suggested. Other possible explainations require a modification to the resonant polaron coupling or a shift in the position of the resonances due to a finite density of states between Landau levels. However, this is not yet able to explain all the experimental observations. 


\section{CHAPTER 4}

\section{MAGNETOPHONON RESONANCE IN GaInAs-InP HETEROSTRUCTUR}

4.1 Introduction

$\begin{array}{lll}4.1 .1 & \text { Overview } & 120\end{array}$

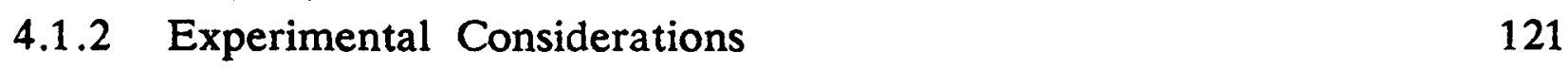

$\begin{array}{lll}\text { 4.2 Optic Phonon Modes in GaInAs-InP } & 121\end{array}$

$\begin{array}{lll}\text { 4.3 The Resonance Position Varies with Carrier Density } & 124 \\ 4.3 .1 \quad \text { In Quantum Wells ..... } & \end{array}$

4.3.2 ..... and in Heterojunctions 127

$\begin{array}{ll}\text { 4.3.3 Supporting Evidence } & 129\end{array}$

$\begin{array}{lll}4.4 & \text { Rotation in a Magnetic Field } & 130\end{array}$

4.5 Resonance Amplitudes and Damping Factors 132

4.6 Discussion of the Resonance Postitions

$\begin{array}{lll}\text { 4.6.1 Possible Explanations } & 136 \\ 4.6 .2 & 137\end{array}$

$\begin{array}{lll}\text { 4.6.2 Reduction in } B_{F} \text { by Carrier Density Dependent Broadening } & 137 \\ \text { 4.6.3 Coupled Plasmon-Phonon Modes } & 138\end{array}$

$\begin{array}{lll}4.7 & \text { Summary } & 141\end{array}$ 


\section{$\underline{4.1 \quad \text { INTRODUCTION }}$}

\section{$\underline{4.1 .1 \quad \text { Overview }}$}

This chapter reports magnetoresistance studies of the 2-D electron gas in GaInAs-InP heterostructures, between $300 \mathrm{~K}$ and $50 \mathrm{~K}$. In this temperature region the electron mobility is limited by optic phonon scattering, thus the nature of phonons coupling to the $2 \mathrm{DEG}$ is very important. The situation in GaInAs-InP is somewhat more complicated than in GaAs-GaAlAs considered in the previous chapter, because with the electrons confined to the ternary material there will be alloy scattering to consider and the optic phonons show mixed modes (Brodsky \& Lucovsky, 1968).

Magnetophonon resonance measurements have previously been reported in heterojunctions (Portal et al. 1984, Gauthier et al. 1988) and multi-quantum wells (Portal et al. 1983), where coupling at lower energy than the GaAs-like LO phonon were found. Comparisons between GaInAs-InP quantum wells (Portal et al. 1983) and GaInAs-AlInAs heterostructures where the 2DEG is formed in the same material (Brummell et al. 1983, Nicholas et al. 1989b) have suggested that coupling to phonon modes from the barrier material may be important.

Phonon energies in GaInAs-InP have also been studied in photoluminescence (Skolnick et al. 1987) and Raman scattering (Pickering 1981, Pearsall et al. 1983), where recent measurements on single quantum wells (Mowbray et al. 1987) have shown frequncies varying with electron concentration, and again below the GaAs-like LO mode.

The present work is concerned with magnetophonon resonance in single GaInAs-InP quantum wells and heterojunctions. The resonance positions are found to decrease markedly with increasing carrier concentration (Leadley et al. 1988a), in a similar way in both types of structure. This behaviour will be compared with that in GaAs-GaAlAs discussed in Chapter 3. In Section 4.4 application of a component of magnetic field parallel to the plane of the 2DEG is found to have similar effects as in the GaAs systems, but differences emerge between samples emphasising the importance of the subband structures. The remainder of the chapter will discuss various possible explainations of these phenomena. 


\subsubsection{Experimental Considerations}

The samples studied were grown by MOCVD and were discussed in detail in Chapter 2. They were provided by two souces: from RSRE - three heterojunctions and a series of single quantum wells of well widths from $100 \dot{A}$ to $800 \AA \dot{A}$; and from Thompson CSF - a series of single heterojunctions. A range of carrier concentrations from 0.8 to $7.6 \times 10^{11} \mathrm{~cm}^{-2}$ was available, and could be varied in a given sample by photoexcitation with a red LED. Persistent photoconductivity did not occur to the same degree as in GaAs-GaAlAs, especially at high temperatures, so in some of the experiments continuous illumination was used.

For samples of high $\mathrm{n}_{\mathrm{s}}$ the magnetoresistance was dominated by Shubnikov-de Haas oscillations at low temperatures. However, at $120 \mathrm{~K}$, where a systematic study was performed, the magnetophonon amplitude was much greater. The oscillations were typically $\sim 5 \%$ of the zero field resistivity and were extracted by subtracting a voltage proportional to the magnetic field, or taking the second derivative of the signal. Carrier concentrations were deduced from the Hall voltage under the same experimental conditions as the magnetophonon resonances were measured.

The other important parameter required is the effective mass which was measured by cyclotron resonance on the same samples, at the same temperature, carrier concentration and amount of LED illumination as the resistance measurements.

\subsection{OPTIC PHONON MODES IN GaInAs-InP}

In alloy materials such as $\mathrm{Ga}_{1-\mathrm{x}} \mathrm{In}_{\mathrm{x}}$ As the frequencies of the possible optic phonon modes vary with the composition as the character of the material changes from that of one constituent compound to the other, in this case GaAs to InAs. In the simplest case there is always just one LO and one TO mode whose frequencies shift monotonically with composition between the frequencies of the optic phonons in the constituent compounds. This is known as one-mode behaviour. Alternatively the alloy may exhibit two-mode behaviour, with two sets of LO and TO phonons being seen for all $x$, whose frequencies remain within the Reststralen band of the respective binary. 
As the composition of $A_{1-x} B_{x} C$ changes from material $A C$ to material $B C$ oscillator strength will be transfered from A-like modes to the B-like modes ie. the splitting $\mathrm{LO}_{\mathrm{A}}-\mathrm{TO}_{\mathrm{A}}$ will decrease as $\mathrm{LO}_{\mathrm{B}}-\mathrm{TO}_{\mathrm{B}}$ increases. At the extreme $(\mathrm{x} \sim 1)$ there will just be a local mode of $\mathrm{A}$ in $\mathrm{BC}$ with negligable oscillator strength. There is a third possibility of mixed-mode behaviour in which two sets of modes are not seen over the entire composition range.

If $\mathrm{Ga}_{1-\mathrm{x}} \operatorname{In}_{\mathrm{x}}$ As displays full two mode behaviour then for $\mathrm{x}=0.53$, where the alloy is lattice matched to InP, the phonon spectrum should contain: GaAs-like LO mode at $272 \mathrm{~cm}^{-1}$ and TO mode at $255 \mathrm{~cm}^{-1}$; and InAs-like LO at $233 \mathrm{~cm}^{-1}$ and TO at $226 \mathrm{~cm}^{-1}$. Each phonon branch $(\lambda)$ will be coupled to the electrons by a Frolich interaction of the same form as in a binary crystal (discussed in Section 1.4.2) but whose strength must be determined from a multi-mode theory. Swierkowski et al. (1978) made such calculations and found the Frollich coupling constant for each mode, $\alpha_{\lambda}$, to be given by:

$$
\alpha_{\lambda}=\frac{e^{2}}{4 \pi \epsilon_{0} \epsilon_{\infty} \hbar}\left[\frac{m^{*}}{2 \hbar \omega_{L \lambda}}\right]^{1 / 2} \frac{\Delta_{\lambda}}{\omega_{L \lambda}}
$$

where

$$
\Delta_{1}=\left(\omega_{\mathrm{L} 1}^{2}-\omega_{\mathrm{T} 1}{ }^{2}\right) \quad \frac{\left(\omega_{\mathrm{L} 1}{ }^{2}-\omega_{\mathrm{T} 2}{ }^{2}\right)}{\left(\omega_{\mathrm{L} 1}{ }^{2}-\omega_{\mathrm{L} 2}{ }^{2}\right)}
$$

$$
\Delta_{2}=\left(\omega_{\mathrm{L} 2}^{2}-\omega_{\mathrm{T} 2}{ }^{2}\right) \frac{\left(\omega_{\mathrm{L} 2}{ }^{2}-\omega_{\mathrm{T} 1}{ }^{2}\right)}{\left(\omega_{\mathrm{L} 2}{ }^{2}-\omega_{\mathrm{L} 1}{ }^{2}\right)}
$$

and for a binary Eqn. 1.29 is recovered by making $\Delta=\omega_{L}^{2}-\omega_{T}{ }^{2}$. This shows how $\alpha$ is reduced for the low energy mode and increased for the higher energy LO mode and in $\mathrm{Ga}_{0.47} \mathrm{In}_{0.53}$ As the calculated values are $\alpha_{\mathrm{GaAs}}=0.050$ and $\alpha_{\mathrm{InAs}}=0.011$. The oscillator strength of the GaAs-like LO mode would then be predicted to be nearly five times larger than the InAs-like mode. Nash et al. 1987 also give values of $\alpha$ form straight interpolations between the corresponding values in the constituent binarys of 0.0449 and 0.0145 respectively. If GaInAs does not display full two-mode behaviour, but has some form of extended phonon band between the GaAs LO and InAs TO a much larger value of $\alpha$ would be calculated - around 0.1 . 
From magnetophonon resonance in bulk GaInAs Nicholas et al. $(1979,1980)$ found only coupling to the GaAs-like mode, although latter work by Sarkar et al. (1985) saw some high field splitting of the peaks and attributed this to the presence of both GaAs and InAs modes. Other experiments eg. band-edge recombination (Goetz et al. 1983); hot carrier luminescence (Shah et al. 1980) only see the higher energy GaAs-mode.

Brodsky \& Lucovsky (1968) first reported mixed mode behaviour in $\mathrm{Ga}_{1-x^{\prime}} \mathrm{n}_{x}$ As from IR reflectivity, finding no InAs-like modes for small $x$. Peasall et al. (1983) studied polarisation selection rules in Raman measurements in a backscattering geometry: with the detection polarised parellel to the incident beam only LO modes should be seen and for perpendicular polarisation only TO modes. They identified a phonon with strong LO character at $270 \mathrm{~cm}^{-1}$ and a true TO mode at $226 \mathrm{~cm}^{-1}$, but between these they only found unpolarised modes which were interpreted as localised vibrations. This suggests that GaInAs does not show full two mode behaviour and the optic phonon spectrum may extend from the GaAs-like LO frequency right down to the InAs-like TO.

In undoped GaInAs-InP quantum wells similar results are seen for low intensity Raman measurements (Mowbray et al. 1987) and in the phonon satellites of excitonic recombination by photoluminescence (Skolnick et al. 1987) with the intensity ratio of the GaAs to InAs modes being around three. By contrast in the doped structures investigated by magnetophonon resonance, mentioned in 4.1 above, and from Raman measurements at high excitation densities, couplings at a whole variety of energies occur.

For a 2-D system there is added complexity over the bulk situation because the confining layers have to be accounted for. Both Mowbray et al. and Skolnick et al. see a strong peak at $357 \mathrm{~cm}^{-1}$ due to the bulk InP LO, and weak structure at $328 \mathrm{~cm}^{-1}$ and $338 \mathrm{~cm}^{-1}$ is also seen in the Raman measurements which may be local modes of $P$ in GaInAs and $\mathrm{Ga}$ in InP, indicating some degree of grading at the interface [estimated as $\sim 40 \AA$ by Skolnick et al. (1986b) ]. For an abrupt junction interface phonons would be expected at $328 \mathrm{~cm}^{-1}$ and $260 \mathrm{~cm}^{-1}$ (Eqn. 3.21) but for a graded interface their oscillator strength will be much reduced. 


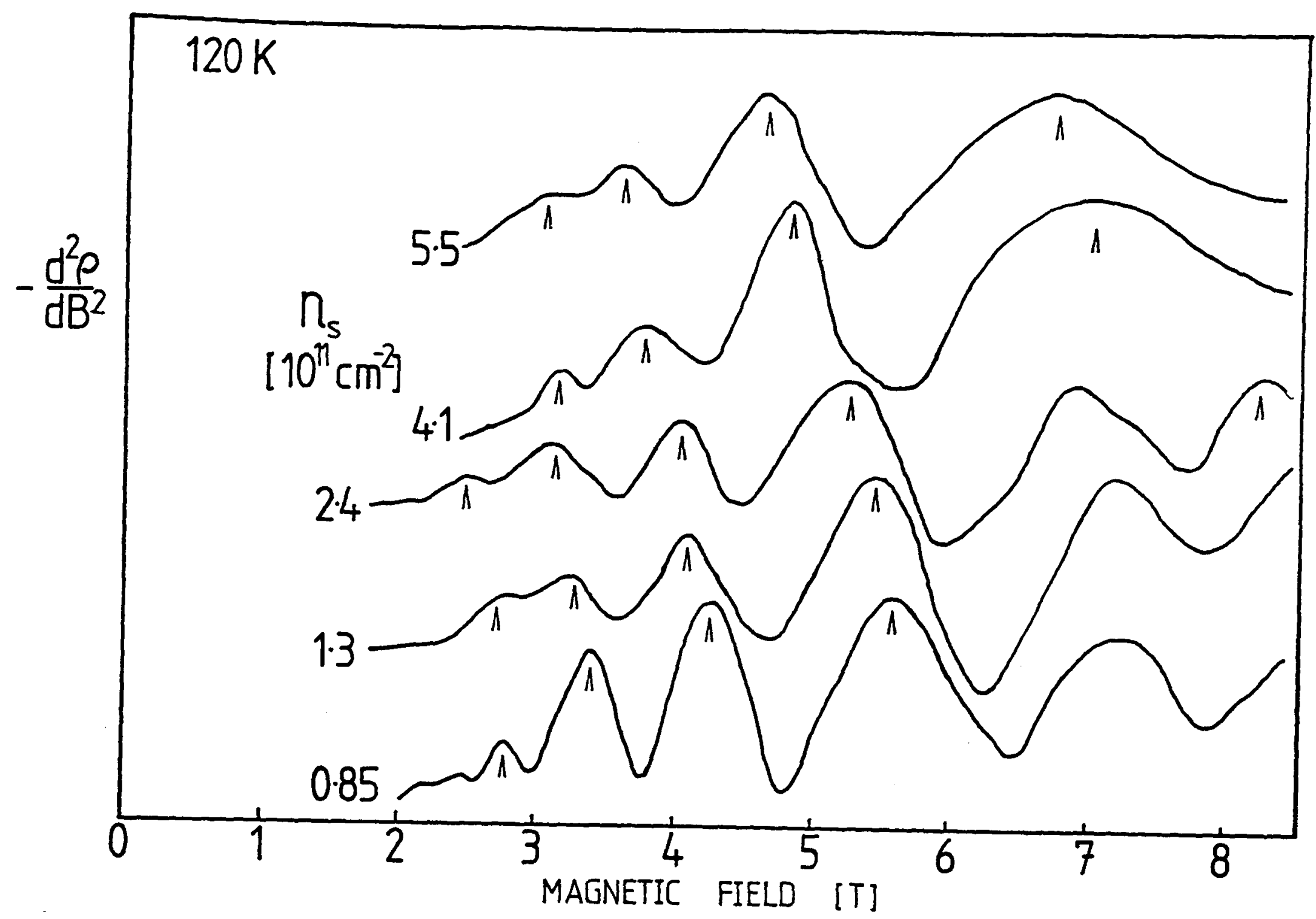

Figure 4.1 Second derivative recordings of magnetophonon resonances in $100 \dot{A}$ GaInAs-InP quantum wells 5599 and 5899 shown for various carrier densities. The marked peaks form series periodic in 1/B.

\subsection{THE RESONANCE POSITION VARIES WITH CARRIER DENSITY}

\subsubsection{In Quantum Wells .....}

Figure 4.1 shows the second derivative of the magnetoresistance for the $100 \AA$ wells with varying carrier densities. The peaks can clearly be seen moving to lower magnetic field as $n_{s}$ increases, with the labelled peaks forming a series periodic in $1 / B$, where the fundamental field changes from $16.8 \mathrm{~T}$ to $13.0 \mathrm{~T}$. The effective mass relevent to the required transitions, $\mathrm{m}_{\mathrm{MPR}}^{*}$, is obtained from cyclotron resonance measurements at the same temperature and correcting the measured mass, $m_{C R}^{*}$, for non-parabolicity and polaron effects using the methods described in the last chapter. In calculating the resonant polaron correction a value of $\alpha=0.05$ has been used for coupling to the GaAs-like mode (but see Section 4.2). It was found that at $120 \mathrm{~K} \mathrm{~m}^{*} \mathrm{CR}$ varied between samples in the range $0.048 \mathrm{~m}_{\mathrm{e}}$ to $0.055 \mathrm{~m}_{\mathrm{e}}$, as given in Table 2.4 , but as $\mathrm{n}_{\mathrm{s}}$ was changed in a given sample $\mathrm{m}^{*}$ remained constant to better than $\pm 0.5 \%$. 


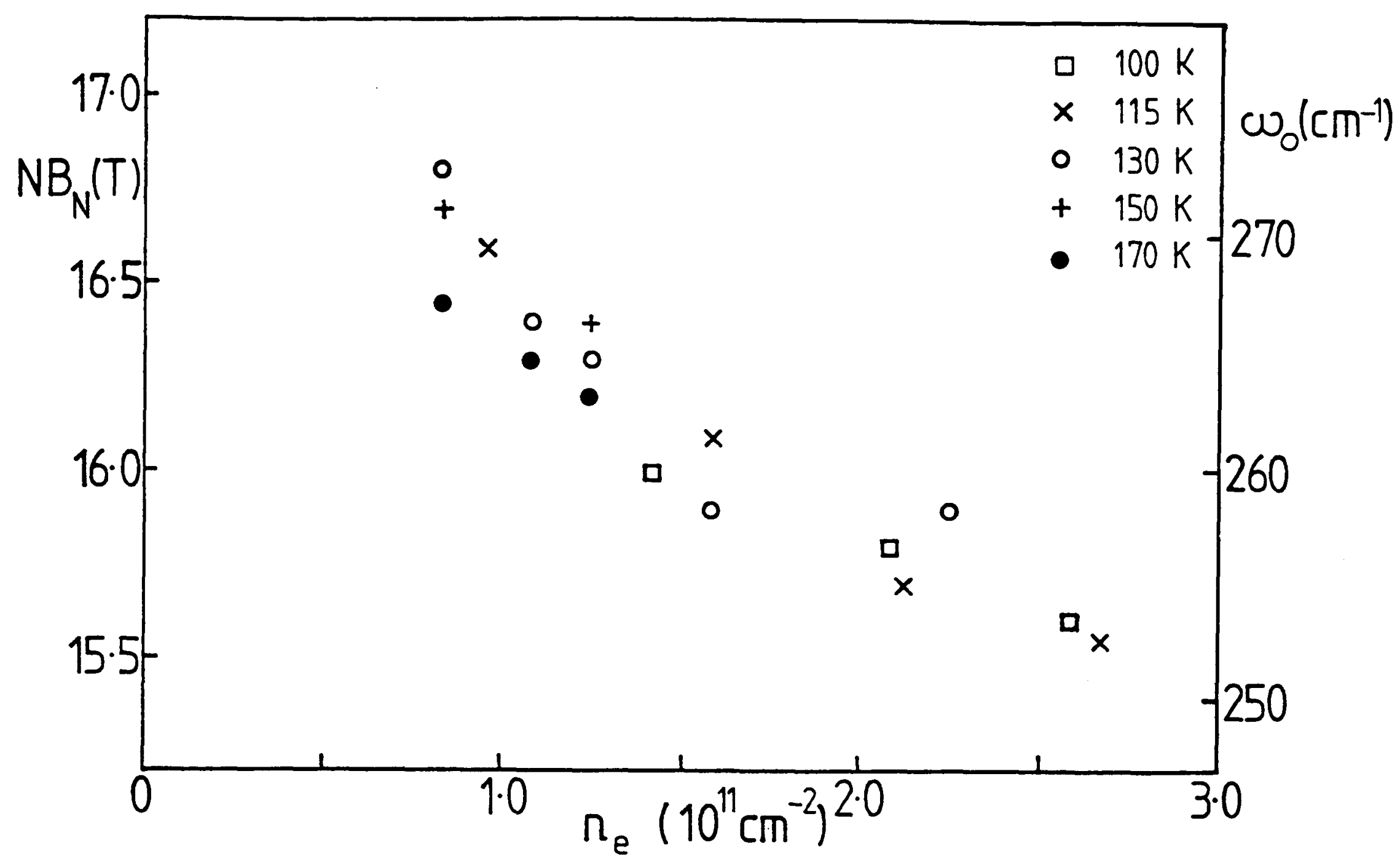

Figure 4.2 Fundamental magnetophonon resonance field $\mathrm{B}_{\mathrm{F}}=\mathrm{NB}_{\mathrm{N}}$ in sample 5599 as a function of carrier density at various temperatures. The corresponding coupling frequencies $\omega_{0}$ are shown on the right.

The resonance positions and corresponding coupling frequencies for the $100 \AA$ undoped quantum well 5599 are shown in Fig. 4.2 which demonstrates a smooth reduction in $B_{F}$ as the carrier concentration is increased and very little dependence on temperature. A similar trend is seen for all the quantum wells studied, but since $\mathrm{m}^{*}$ varies the measured resonance positions show discontinuities between samples. However, inclusion of the measured effective mass and plotting the apparent phonon frequency in Fig. 4.3 removes the divergences and shows a remarkable variation with carrier density: right from the GaAs-like LO at $272 \mathrm{~cm}^{-1}$ down to the InAs-like TO at $226 \mathrm{~cm}^{-1}$. This figure shows data from all the samples studied and includes caes where $n_{s}$ has been increased both by persistent photoconductivity and under continuous illumination from a red LED. Note the smooth curve from one sample to the next demonstrating that population of the well by doping or photoexcitation is equivalent. 


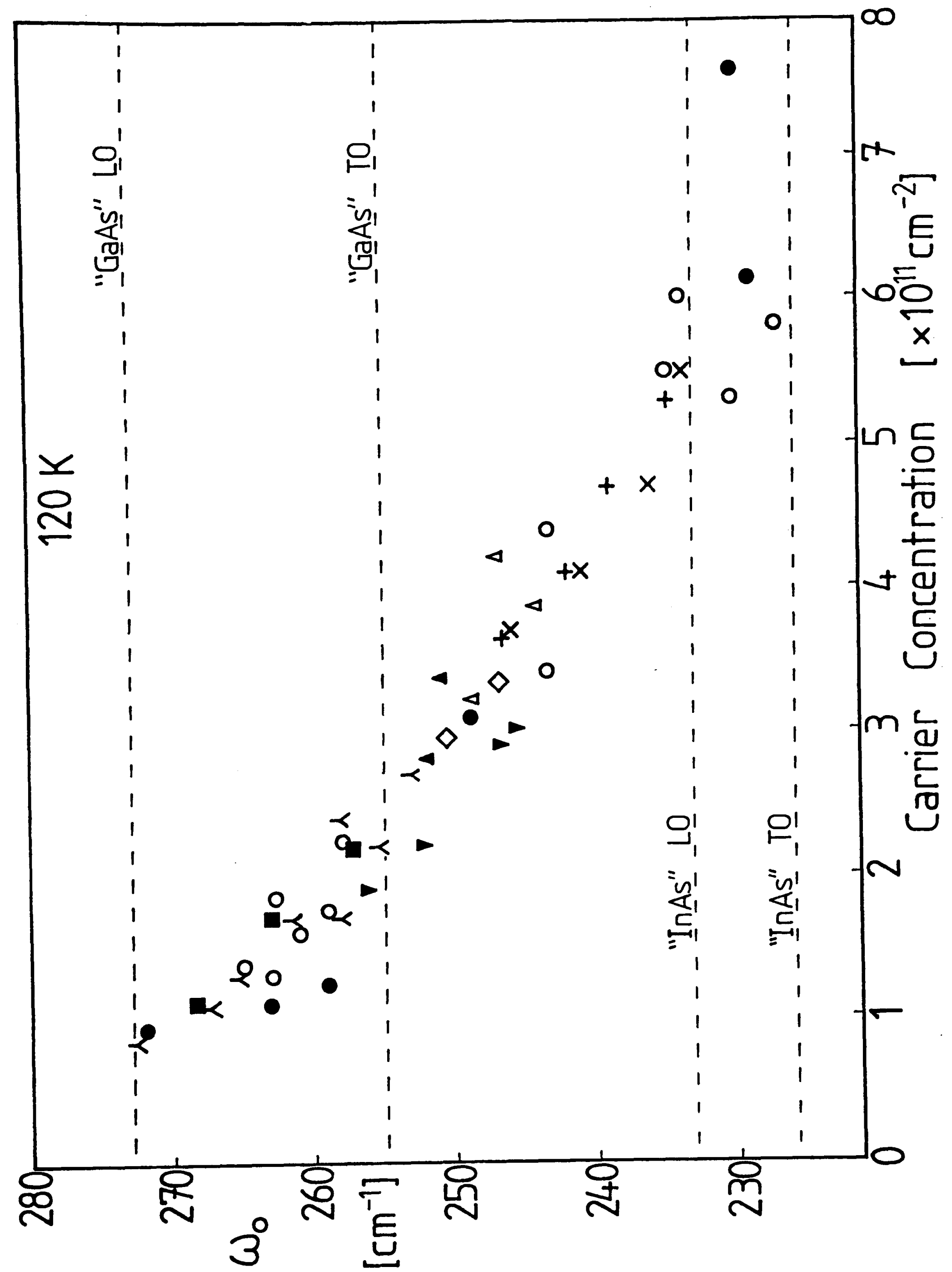

Figure 4.3 Carrier concentration dependence of the apparent phonon frequencies deduced from magnetophonon resonance in GaInAs-InP heterostructures.

Quantum wells: $x,+, \lambda \& \diamond-100 \dot{A} ; \nabla-200 \dot{A} ; \triangle, \Delta-300 \dot{A}$;

Heterojunctions: from RSRE •, from Thompson CSF 0 .

- multi-quantum wells of Portal et al. 
There is an additional peak seen in low density samples (see Fig. 4.1) at around $7 \mathrm{~T}$ which forms part of a second series corresponding to a phonon of frequency $\sim 350 \mathrm{~cm}^{-1}$. This is close to the bulk InP LO mode and is evidence for scattering by a remote phonon, as proposed by Hess \& Vogl (1979), reported by Portal et al. 1983 and discussed theoretically by several authors (Lassnig \& Zwadski 1984a, Sawaki 1986).

The mechanism of coupling electrons in GaInAs to the InP LO mode requires some explanation. InP is a more polar material than GaInAs and so the InP optic phonons will couple strongly to electrons. The electric field of the InP LO will only be partly screened at the 2DEG in GaInAs and coupling could be enhanced by barrier penetration of the electronic wavefunction. That a mode at $328 \mathrm{~cm}^{-1}$ is not identified may suggest that the interface is not particularly abrupt and so the interface modes are not well defined. The grading of the interface means $P$ atoms will be distributed across the region, allowing propagation of vibrations around the InP LO frequency across the barrier. Portal et al. (1983) report that the amplitude of oscillations due to InP increases in narrower wells and is comperable to the GaAs like mode for $80 \AA$ wells and we also only observe this peak in narrow wells.

Only the $\mathrm{N}=3 \mathrm{InP}$ peak is observed and this tends to be rather broad and becomes obscured by the $N=2$ peak of the GaAs series as the latter shifts down in field with increasing $n_{s}$. Consequently it is difficult to state categorically whether the coupling frequency of the InP mode changes with $n_{s}$. The impression gained from Fig. 4.1 is that it is reduced but at a slower rate than the GaAs series.

\subsection{2 $\ldots \ldots$ and in Heterojunctions}

The remarkable trend of Fig. 4.3 is not perculiar to the quantum well samples: included in the figure are circles corresponding to resonance positions for GaInAs-InP heterojunctions, following exactly the same curve, regardless of their detailed structure!

The Razeghi heterojunctions were studied in detail and Fig. 4.4 shows the measured positions of the $\mathbf{N}=2$ resonance as a function of carrier density. This does not show the same monotonic dependence seen in Fig. 4.3, where differences in $\mathrm{m}^{*}$ have been accounted for. However, the data falls into three distinct groups determined by the 
number of electric subbands occupied at low temperature [deduced from the Shubnikov-deHaas and parallel field depopulation measurements described in Chapter 6]. Of particular note are samples 82 and 93 which show a large difference in magnetophonon resonance position, with two and three subbands populated respectively and yet in both $\mathrm{n}_{\mathrm{s}} \sim 5 \times 10^{11} \mathrm{~cm}^{-2}$. By contrast on Fig. 4.3 they both lie on the universal curve. The subband energies were mentioned as important in the GaAs-GaAlAs magnetophonon resonance of Chapter 3 and in Section 4.4 this will be seen to influence the rotation dependence of the oscillations.

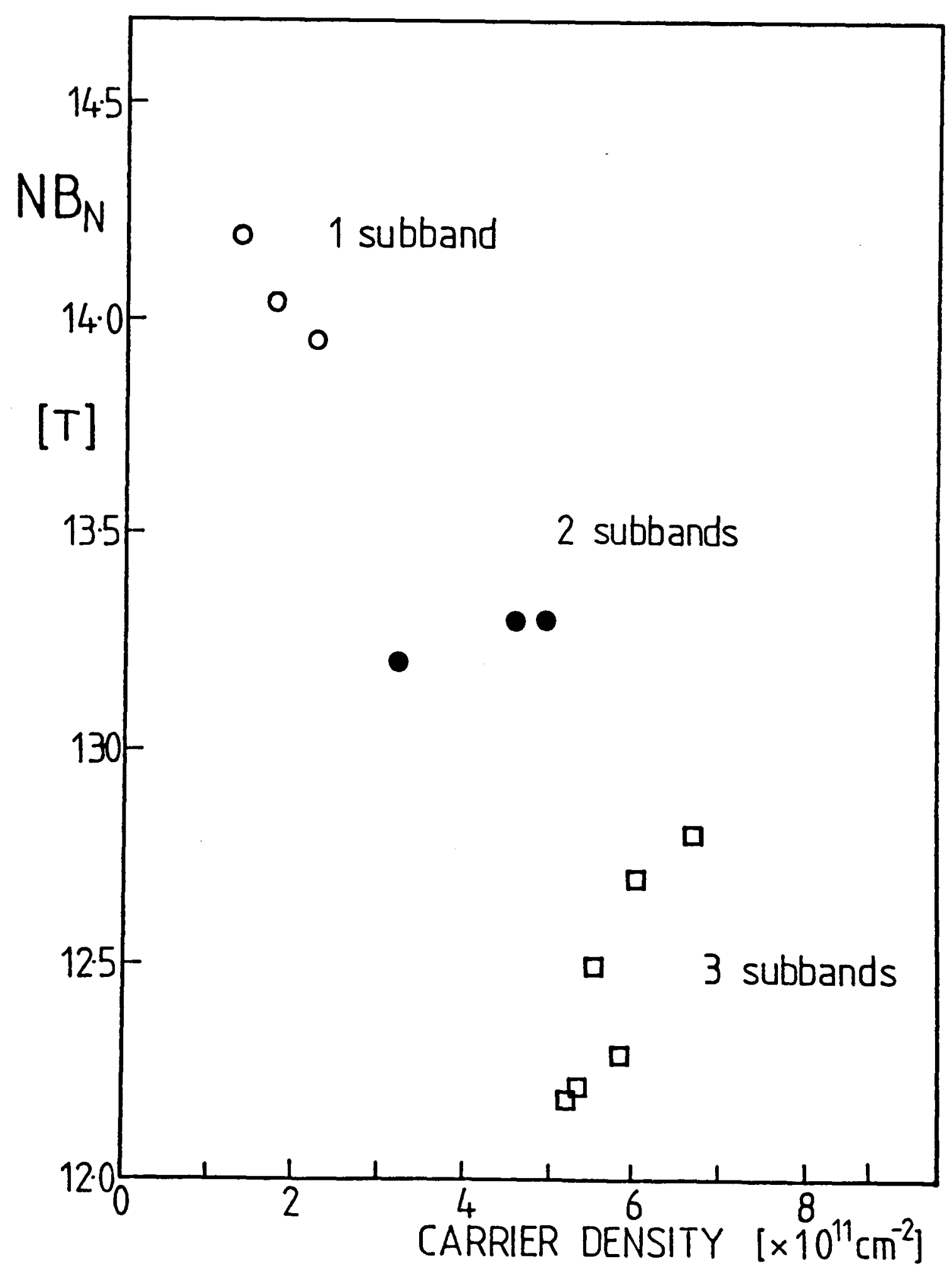

Figure 4.4 Fundamental magnetophonon resonance field $B_{F}=N B_{N}$ in GaInAs-InP heterojunctions as a function of $\mathrm{n}_{\boldsymbol{s}}$. Notice how the data form groups. determined by the number of subbands occupied at low temperature. 


\subsubsection{Supporting Evidence}

These results are in accord with previous measurements on GaInAs based systems. The GaInAs-InP multi-quantum wells studied by Portal et al. (1983) are included on Fig. 4.3 as boxes and fit exactly. In GaInAs-AlInAs, where only the barrier material has been changed, two apparently contradictory sets of results have been publisised. Brummell et al. (1983) studied high density heterojunctions $\left(\mathrm{n}_{\mathrm{s}} \sim 7 \times 10^{11 \mathrm{~cm}} \mathrm{c}^{-2}\right)$ and found coupling at $239 \mathrm{~cm}^{-1}$ which was attributed to the InAs-like mode being able to propagate in both materials at approximately the same energy [this would also give rise to strong interface modes ]. By contrast Nicholas et al. (1989b) used a low density multi-quantum well $\left(\mathrm{n}_{\mathrm{s}} \sim 1.8 \times 10^{11} \mathrm{~cm}^{-2}\right)$ where coupling was observed at $272 \mathrm{~cm}^{-1}$, near the GaAs LO mode, and also at $\sim 355 \mathrm{~cm}^{-1}$ with the AlAs-like barrier phonons. These two results lie slightly above the curve of Fig. 4.3 but show just the same amount of decrease of $\omega_{L}$ with $\mathrm{n}_{\mathrm{s}}$. Thus it would appear that the change in resonance position with carrier concentration is basically determined by the material in which the $2 \mathrm{DEG}$ is confined, with the barriers maybe making a small adjustment to the absolute values.

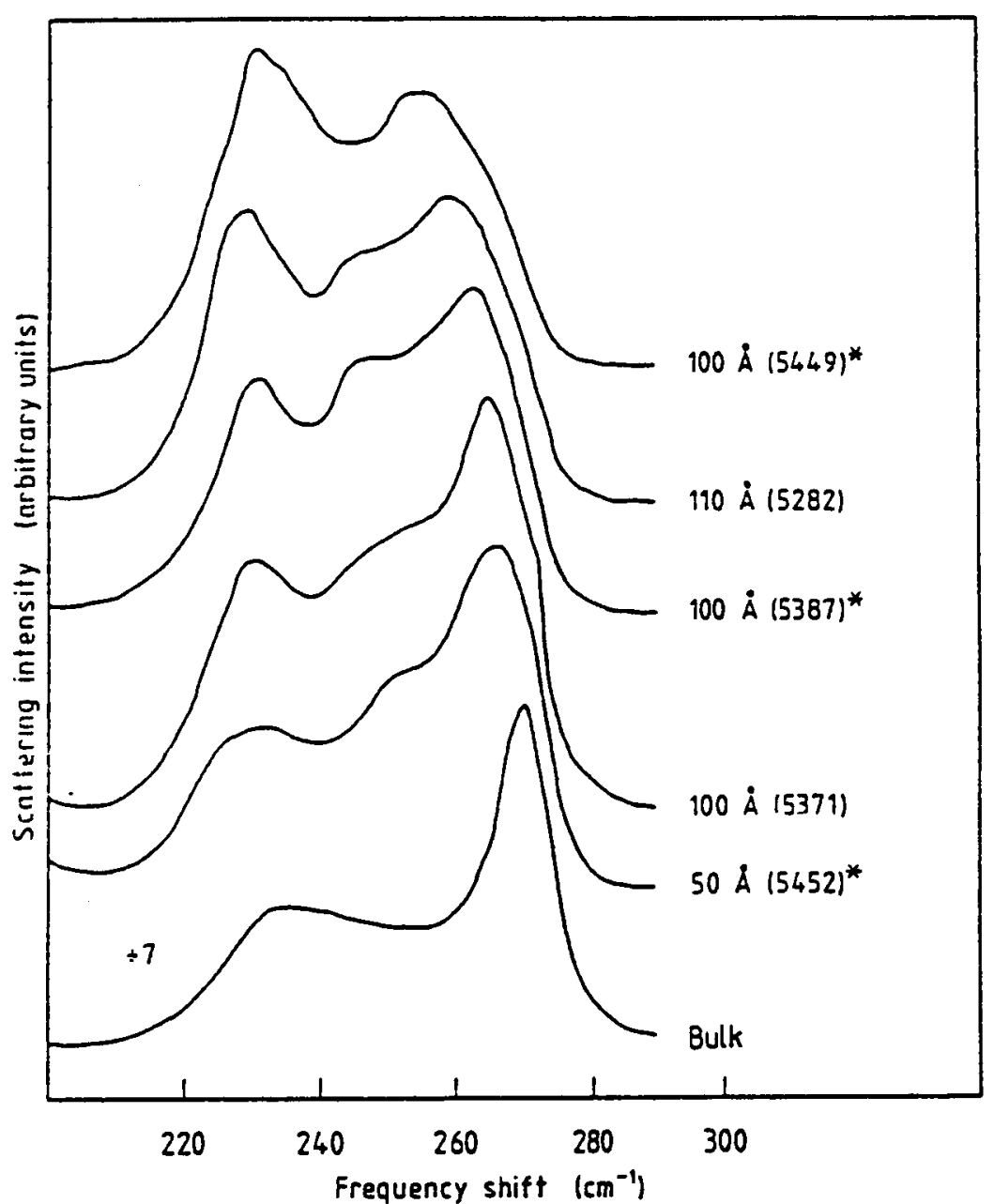

Figure 4.5 Raman spectra for a range of quantum wells showing the shift of the GaAs-like LO mode to lower energies accompanied by its broadening and an increase in strength of the InAs-like mode. The bottom spectrum is from bulk GaInAs for comparison. 
Raman scattering measurements by Mowbray et al. (1987) on similar GaInAs-InP quantum wells to those studied here also show a change in frequency of the phonon modes. As the laser power is increased the GaAs-like LO mode shifts down from $267 \mathrm{~cm}^{-1}$ to $255 \mathrm{~cm}^{-1}$, at the same time broadening and transfering oscillator strength to the InAs-like mode. Increasing the laser power will excite more carriers into the well, but it is difficult to make quantitative measurements of $n_{s}$ in these experiments. Under high power a similar change between samples is seen and this is illustrated in Fig. 4.5. It is noticable that the $2 \mathrm{D}$ behaviour is very different to that seen in bulk where the GaAs-like LO mode appears as a sharp feature.

\subsection{ROTATION IN A MAGNETIC FIELD}

On rotation of the samples in the magnetic field the resonance positions move to higher field, showing that the resonances come from two dimensional electrons. However, a striking difference is now seen between the different structures: in the quantum wells the resonance positions closely follow $\mathrm{B}_{\mathrm{F}} / \cos \theta$ and the oscillation

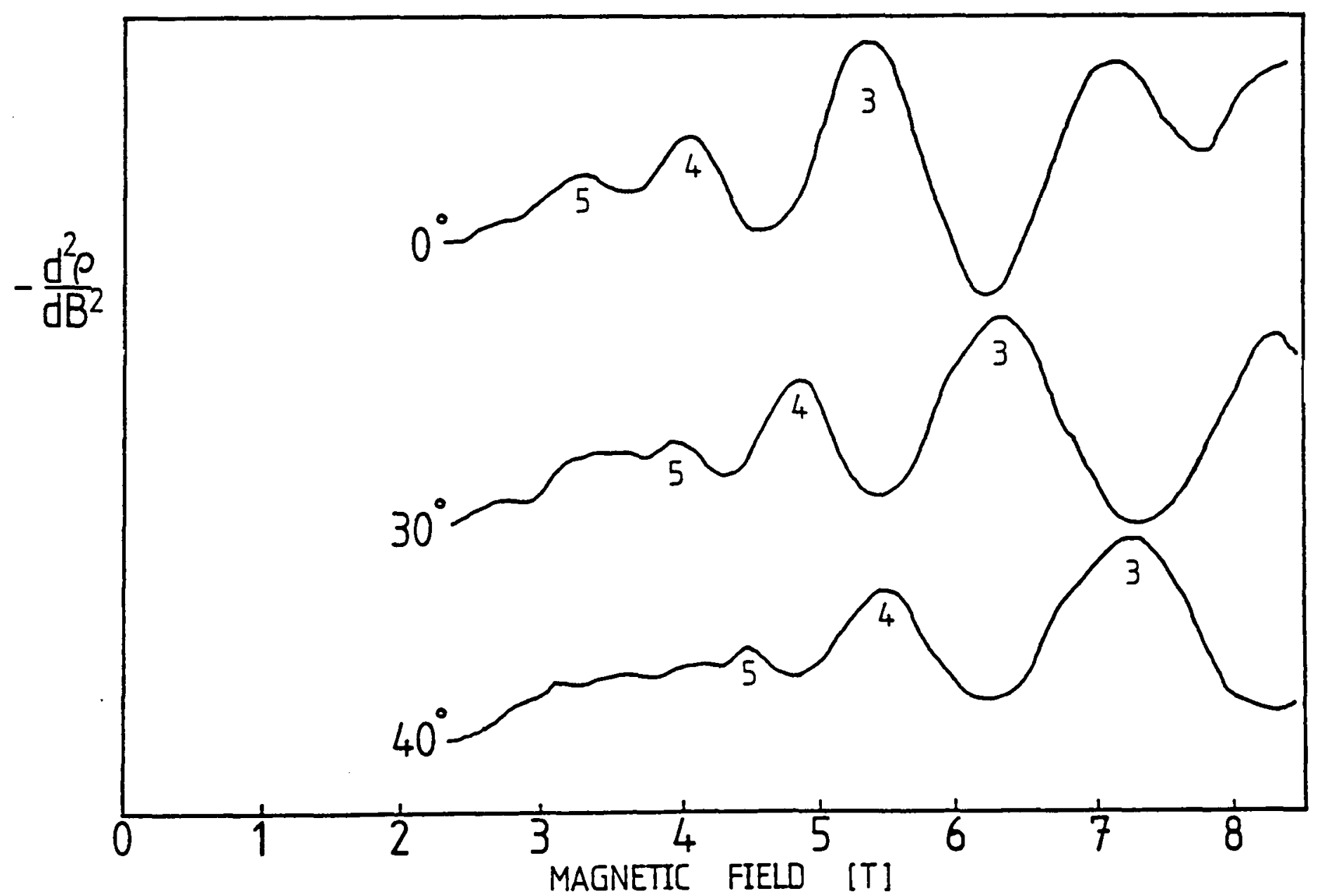

Figure 4.6 Second derivative of magnetoresistance in a $100 \dot{A} \mathrm{QW}$ for various angles between the magnetic field and the normal to the 2DEG showing the two-dimensional nature of the oscillations and a constant amplitude. 
amplitudes remain constant at all angles (Fig. 4.6); the heterojunctions show a different behaviour with the amplitude falling dramatically (Fig. 4.7) and the resonance positions deviating from $B_{R}=B_{F} / \cos \theta$. This latter effect is very similar to that seen in GaAs-GaAlAs heterojunctions, but the rate of decrease is not as fast and apparently depends on the carrier density and subband separations. In sample 83, with the lowest carrier concentration, and showing coupling at the GaAs-like LO frequency, the amplitude hardly changed on rotation whereas in the higher carrier concentration samples it halved by $25^{\circ}$. In fact the above generalisation is not strictly true, since the wide quantum wells $(>300 \dot{A})$ behaved rather like heterojunctions with the amplitude falling on rotation. The important factor would thus appear to be the difference between the Fermi energy and the energy of higher subbands, which will be largest for narrow wells and low density samples.

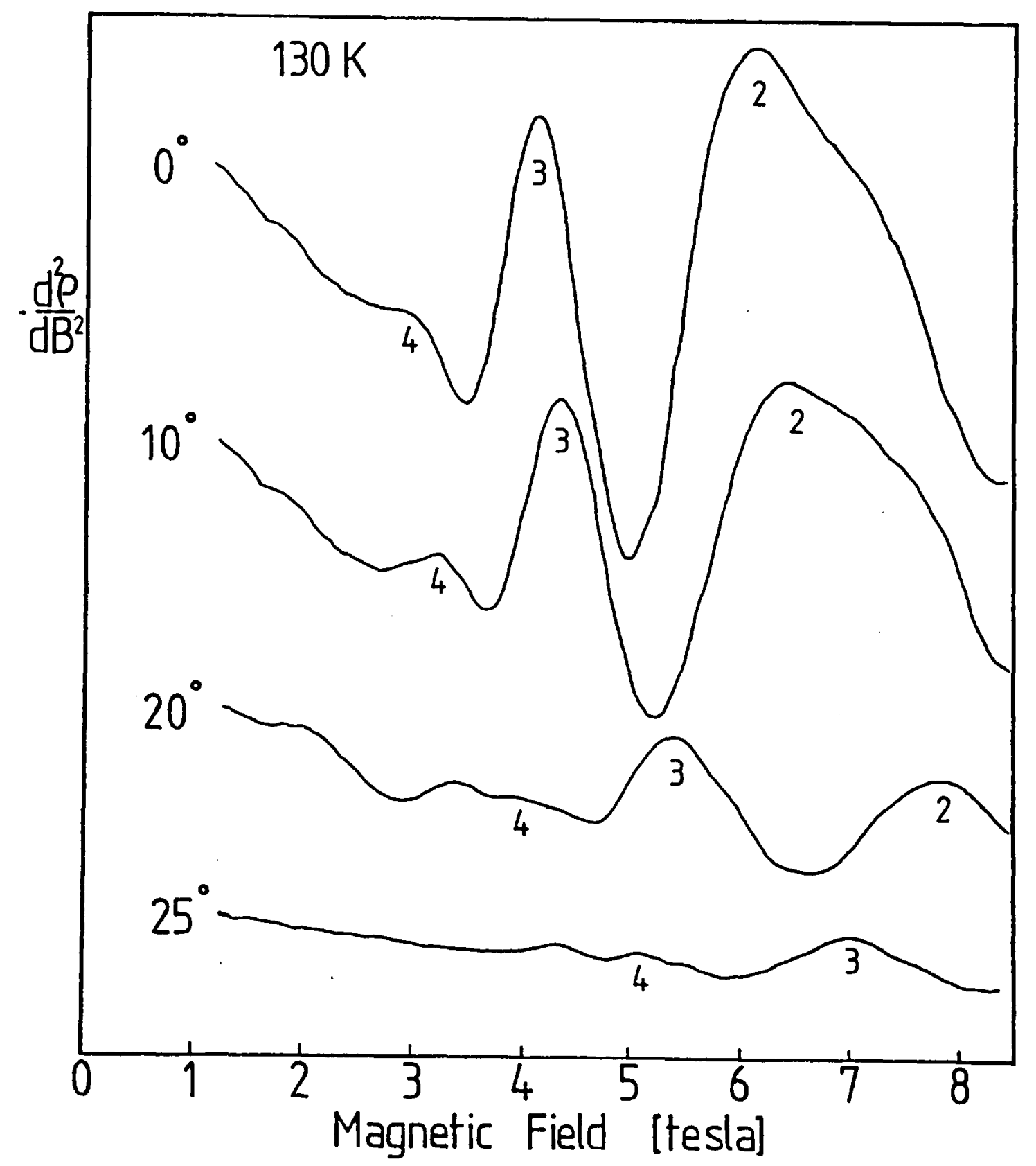

Figure 4.7 Magnetophonon resonance in a heterojunction under rotation in a magnetic field showing the oscillations moving to higher total field and a dramatic loss in amplitude by only $25^{\circ}$. 


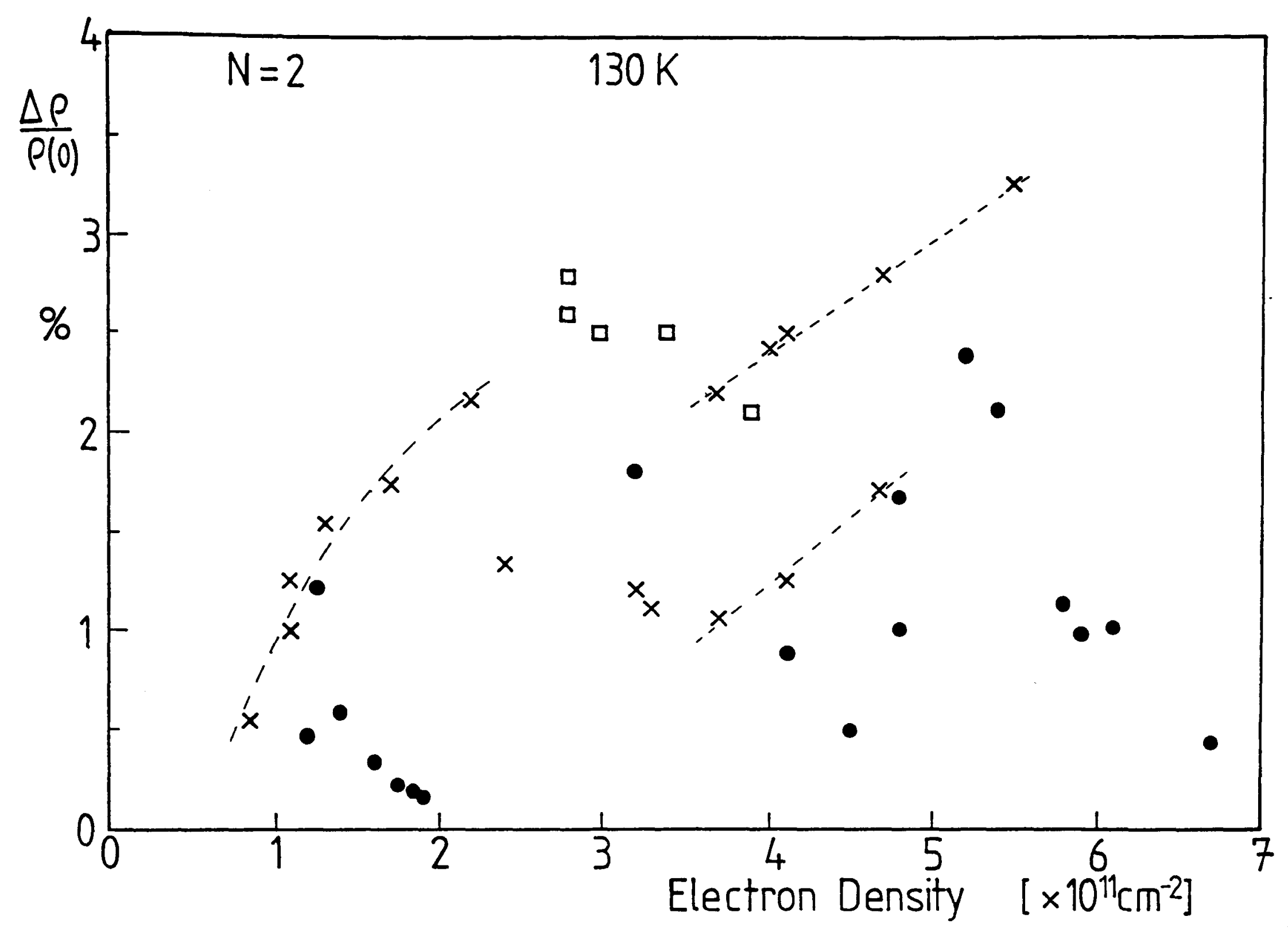

Figure 4.8 Normalised amplitude of the $\mathrm{N}=2$ magnetophonon resonance in

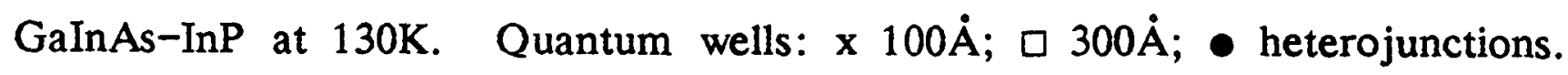
(Dashed lines indicate the change on photoexcitation in a given sample)

\subsection{RESONANCE AMPLITUDES AND DAMPING FACTORS}

The strength of the magnetophonon resonance amplitudes in GaInAs-InP does not seem to follow a similar universal curve to that found for GaAs-GaAlAs, as seen from the $N=2$ amplitudes in Fig. 4.8. This is not unduly suprising condsidering that in Chapter 3 the amplitude was found to be determined by the damping factor ie. the amount of scattering in the sample, and in that the structure varies much more between these GaInAs-InP samples than for the GaAs based system. Also alloy scattering will need to be considered: as this ultimately dominates the mobility in GaInAs at low temperature (Walukiewicz et al. 1984). It is expected to show as much influence on the magnetophonon amplitude as remote impurity scattering did for GaAs-GaAlAs modulation doped heterojunctions. 


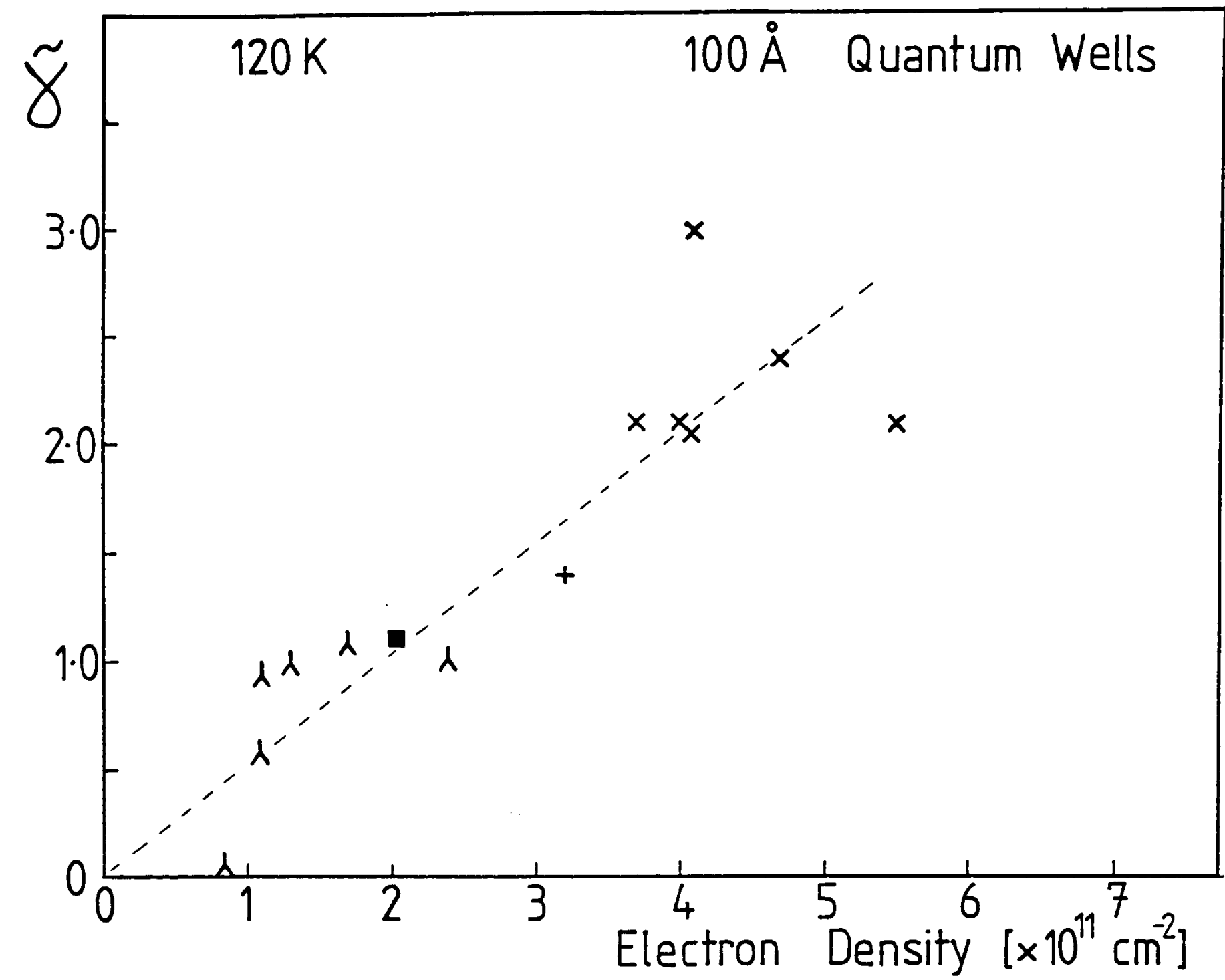

Figure 4.9 Damping factor $\left(\tilde{\gamma}_{23}\right)$ shown as a function of carrier concentration at $120 \mathrm{~K}$ for $100 \AA$ quantum wells. $\curlywedge 5599 ;$ x 5899; + 5868; 5371 .

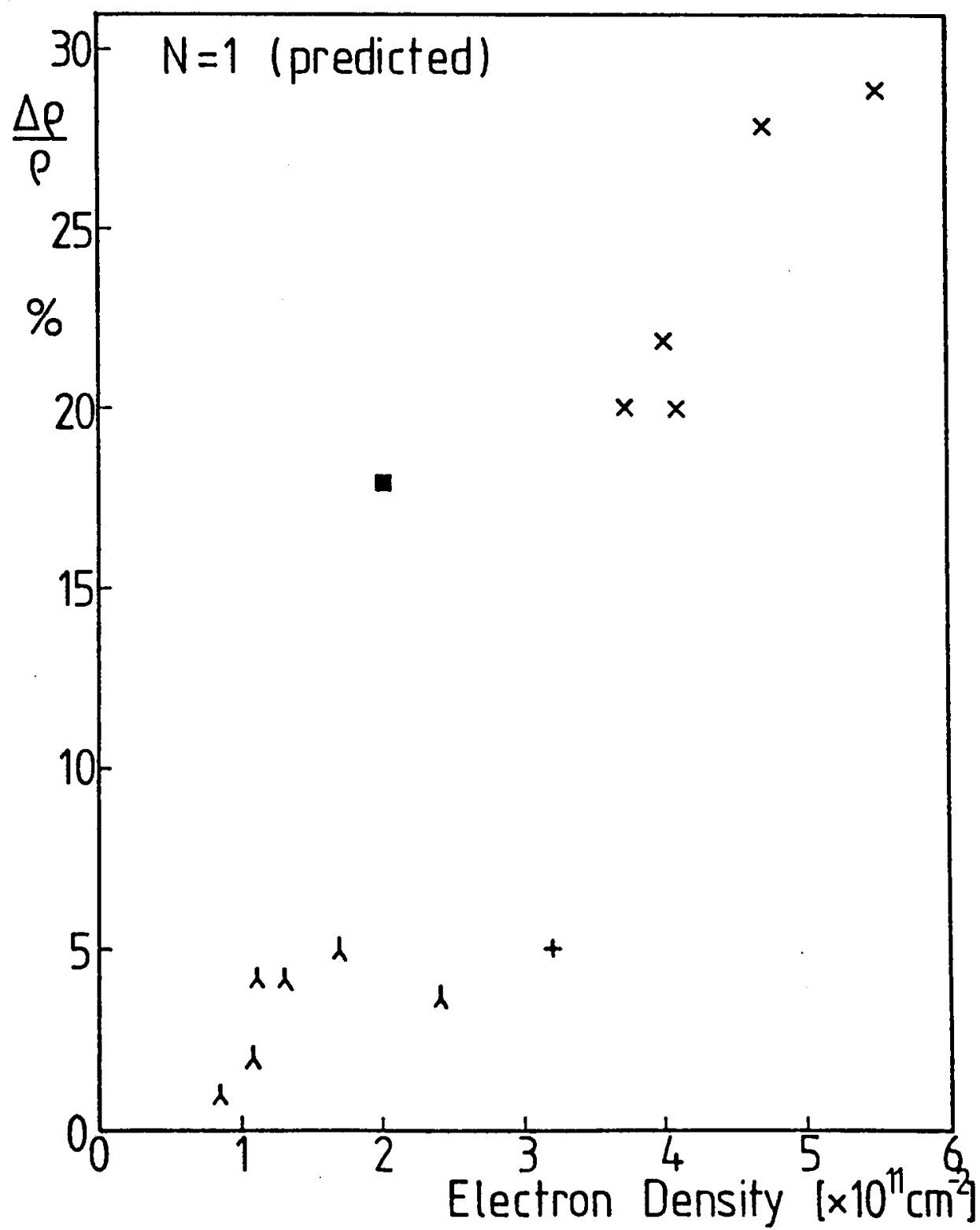

Figure 4.10 Predicted amplitude for the fundamental magnetophonon resonance in the . $100 \dot{A}$ quantum wells at $120 \mathrm{~K}$, shown as a function of $n_{s}$. [Key as Fig. 4.9] 
In Fig. 4.8 the amplitude in each $100 \AA$ quantum well shows an increase with $n_{s}$ as the samples are photoexcited (connected by dashed lines), whilst $\tilde{\gamma}$ stays roughly constant (Fig. 4.9). The points for $\mathrm{n}<3 \times 10^{11} \mathrm{~cm}^{-2}$ are from undoped wells and those at higher densities are from modulation doped wells with $100 \AA$ spacer layers. Of these 5868 has a doping density of $1 \times 10^{17} \mathrm{~cm}^{-3}$ and this is doubled in 5899 explaining the larger $\tilde{\gamma}$ in the latter. Although $\tilde{\gamma}$ stays approximately constant within a given sample a reasonably good fit for all these $100 \dot{A}$ quantum wells may be obtained by the straight line:

$$
\tilde{\gamma}=0.46 \mathrm{n}_{\mathrm{s}}\left(\times 10^{11} \mathrm{~cm}^{-2}\right)
$$

Combining the measured values of $\Delta \rho(2) / \rho_{0}$ and $\tilde{\gamma}$ the amplitude of the $N=1$ resonances can be predicted and are seen, in Fig. 4.10, to increase with $n_{s}$ in a reasonably smooth fashion.

By contrast no sensible trends emerge for the $\mathrm{N}=2$ heterojunction peak amplitudes and looking at the damping factor in Fig. 4.11 is of little help! However, just as seen in very different GaAs-GaAlAs samples, plotting the normalised $\mathbf{N}=1$ magnetophonon

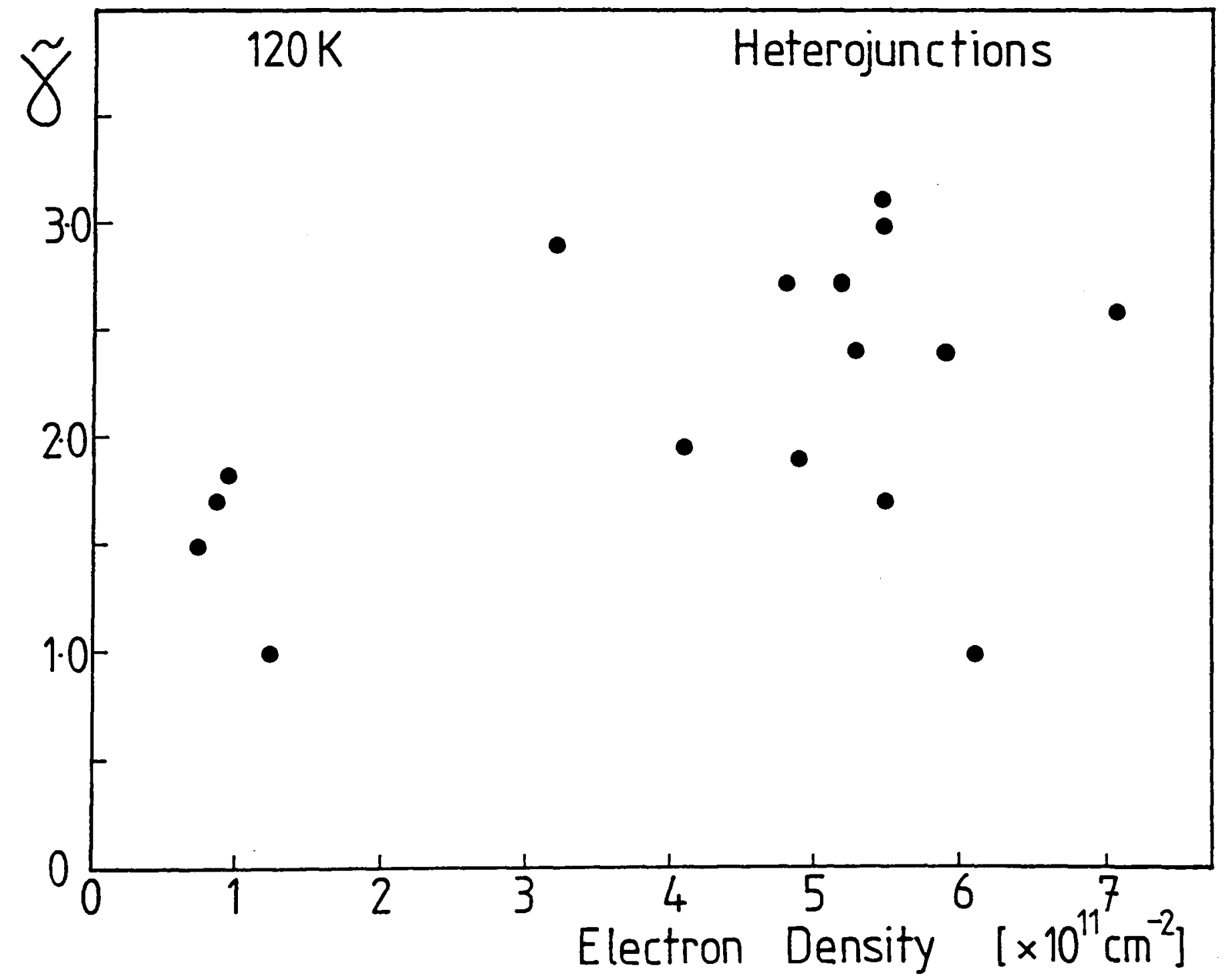

Figure 4.11 Damping factor $\left(\tilde{\gamma}_{23}\right)$ shown as a function of carrier concentration at $120 \mathrm{~K}$ for the heterojunctions. 
amplitude measured at $120 \mathrm{~K}$ against the mobility measured at $4.2 \mathrm{~K}$ in Fig. 4.12 shows a remarkable correlation. Even the two pieces of both samples 82 and 52 which give anomalous results in all other respects fit neatly into the general trend. This really reinforces the view that there exists a fundamental link between the magnetophonon amplitude and $\mu_{4 \mathrm{~K}}$.

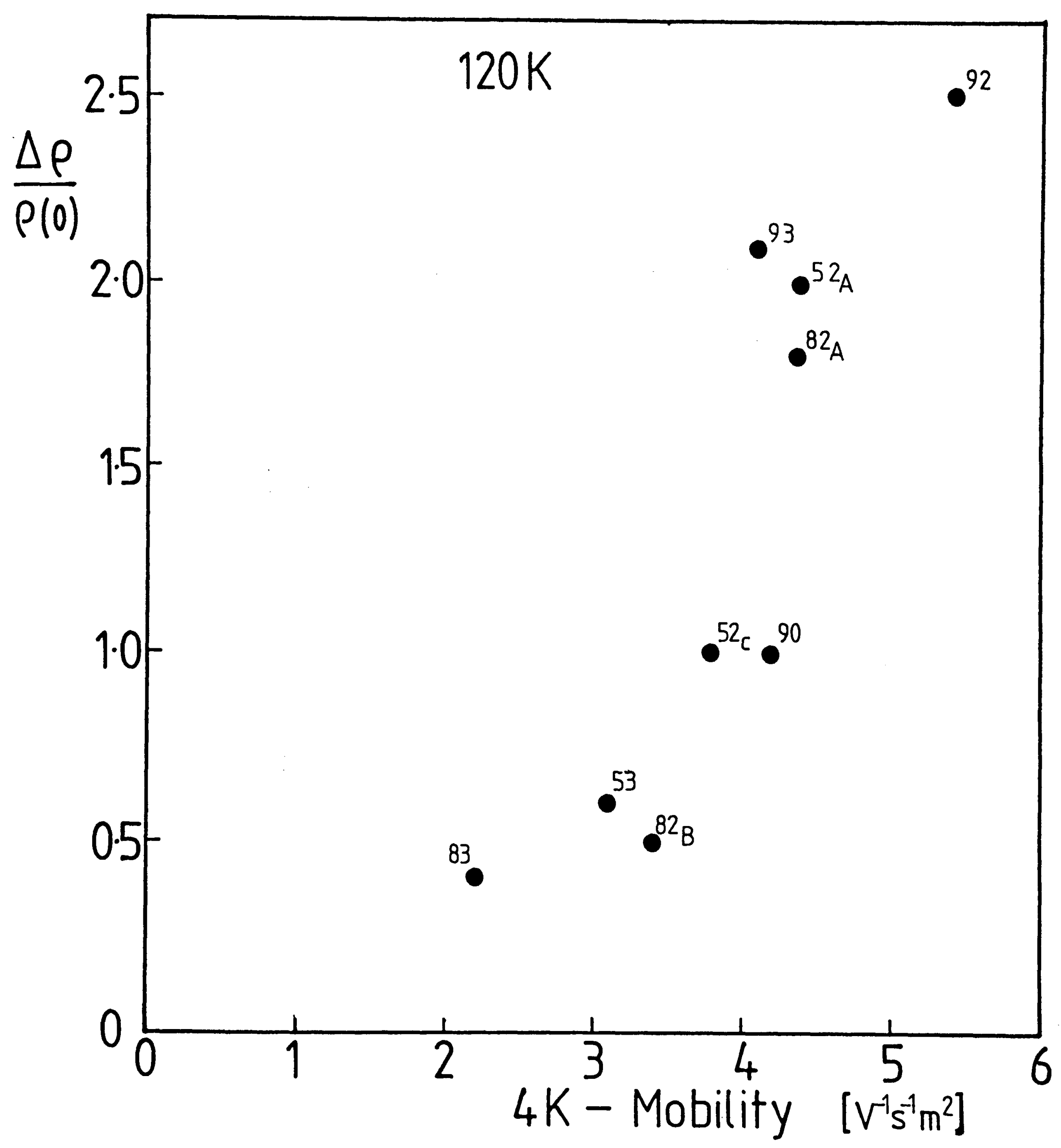

Figure 4.12 Predicted $N=1$ amplitude for the Razeghi heterojunctions at $120 \mathrm{~K}$, showing a remarkable correlation with the mobility measured at $4.2 \mathrm{~K}$. 


\subsection{DISCUSSION OF THE RESONANCE POSITIONS}

\subsubsection{Possible Explanations}

It has previously always been assumed that the electrons of the 2DEG couple to LO phonons. In GaInAs this should be the GaAs-like LO mode at $273 \mathrm{~cm}^{-1}$, as its oscillator strength is much greater than that of the InAs-like mode. So why does the coupling frequency change with $\mathrm{n}_{\mathrm{s}}$, as suggested by Fig. 4.3 ?

Of the possible explanations given for the low coupling frequency in GaAs-GaAlAs the following may also be relevent to the present system:-

(a) Strong screening of the electric field associated with the GaAs-like LO mode would reduce its frequency. The amount of screening will increase with $n_{s}$ and so the phonon energies should show the required trend. However, the frequency could only be reduced to that of the TO phonon of the same mode and hence the full range of resonance positions would only be obtained if GaInAs does not display two mode behaviour. The Raman results of Fig. 4.5 certainly suggest that the optic phonon spectrum really is changed on increasing $n_{s}$.

(b) Occupation effects or the increased degeneracy of the system, which has been found to strongly reduce polaron coupling in GaAs-GaAlAs, may also reduce the frequency of the LO phonon.

(c) Interface mode coupling can not describe the behaviour since the lowest frequency phonon would be at $260 \mathrm{~cm}^{-1}$ and the interfaces are probably not well defined. More likely are local modes in the region of the interface, but these are at much too high a frequency.

(d) Similarly penetration of the barriers will only increase the observed resonance position.

(e) Interaction may be with coupled plasmon-LO-phonon modes. If these include coupling to intersubband excitations a reduction in frequency with $n_{s}$ may occur and this will be treated in more detail in Section 4.6.3.

(f) Coupling may actually be to the GaAs-like LO mode, but the resonance position may be shifted by damping of the oscillations from Gaussian broadened levels. 


\subsubsection{Reduction in Coupling Frequency by $\mathrm{n}_{s}$ Dependent Broadening}

Warmenbol (1989) has used the momentum balance equation approach (Eqns. 2.6 \& 2.25) to model this situation by noticing that Gaussian broadening leads to a decrease in resonance position which is enhanced if a constant background term is included. They assumed a level broadening that increases with carrier density like:

$$
\Gamma(\mathrm{meV})=4 \mathrm{n}_{\mathrm{s}}\left(\mathrm{x}^{\left.10^{11} \mathrm{~cm}^{-2}\right)}\right.
$$

and the results are shown in Fig. 4.13 for different amounts of density of states in the flat background. The decrease in resonance position with $n_{s}$ appears to depend on both the $\%$ background and $\Gamma$, with $\sim 30 \%$ required for broadening given by Eqn. 4.3.

So does this explain the results? It is an interesting model but there remain a few problems:

(i) The cyclotron resonance position remains constant as $n_{s}$ is changed at $120 \mathrm{~K}$ where the magnetophonon measurements are made. It is not clear how (or if) this should

Sheet electron density $\mathrm{Ne}\left(10^{11} \mathrm{~cm}^{-2}\right)$

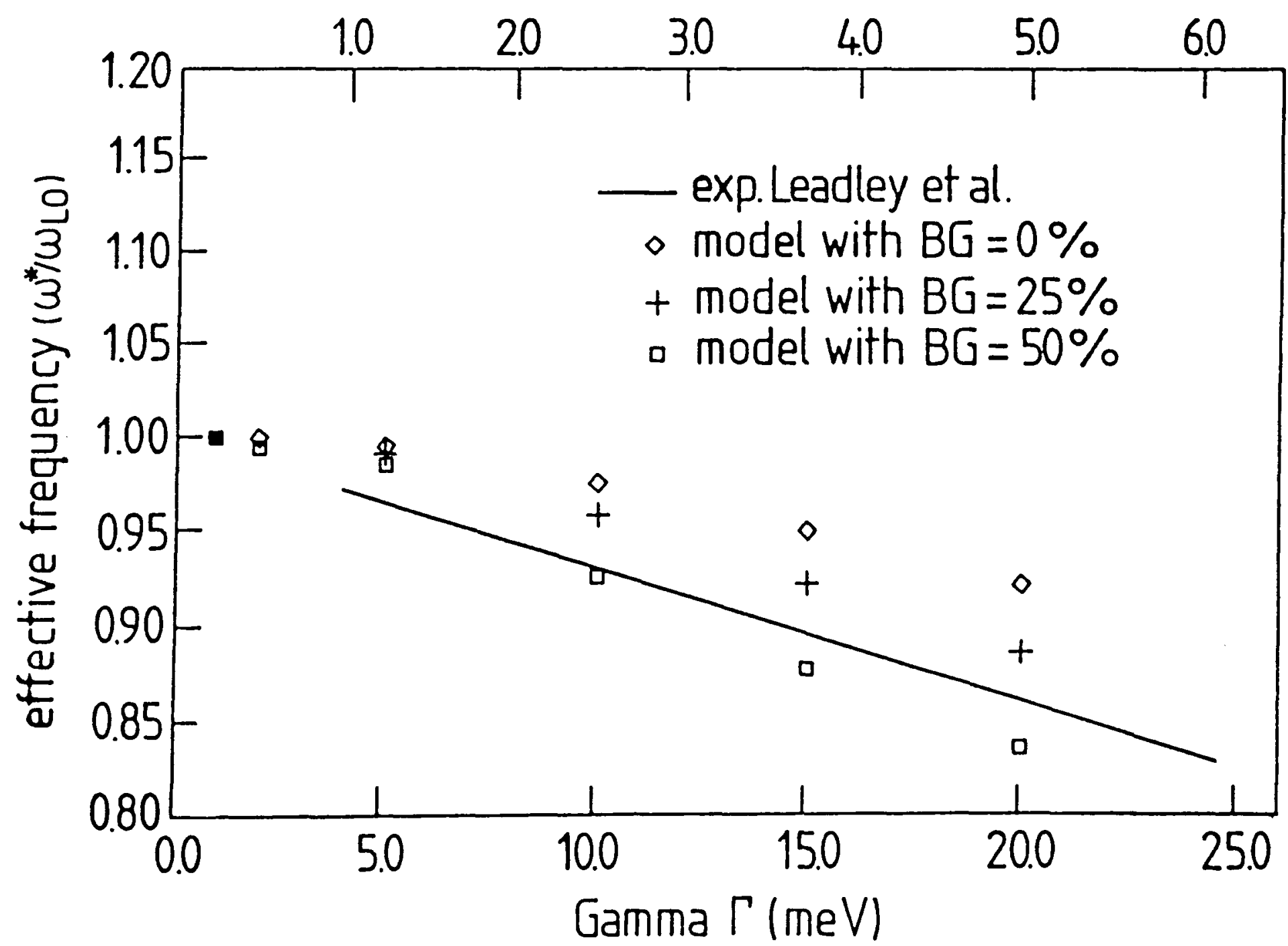

Figure 4.13 Magnetophonon coupling frequency calculated by Warmenbol for different background densities of states assuming $\Gamma$ depends on carrier density. These experimental results are shown as the straight line. 
be affected in this model: probably not much well away from the polaron region, but Warmenbol et al. have only considered cyclotron resonance to be measured at $4.2 \mathrm{~K}$ where the levels are narrowed by screening.

(ii) Although $\tilde{\gamma}$ increases almost linearly with $n_{s}$ in the $100 \dot{A}$ quantum wells this is certainly not true in the heterojunctions (Fig. 4.11) or wider wells, all of which fit equally well on the curve Fig. 4.3, but would not fit on Fig. 4.13 according to the model.

(iii) From Eqns. $2.20 \& 3.9$ the broadening of Eqn. 4.3 can be converted to an experimental damping factor of $\tilde{\gamma} \approx 0.76 \mathrm{n}_{\mathrm{s}}$, ie. a factor of $\sim 2$ larger than that observed experimentaly in the $100 \AA \dot{A}$ quantum wells. Thus if the smaller experimental damping were used an even larger \% background density of states would be required to fit the data.

(iv) The shift in resonance position is associated with transport properties, but does this model also account for the Raman spectra which show the same qualitative shifts?

\subsubsection{Coupled Plasmon-Phonon Modes}

The idea of coupled LO-phonon-plasmon modes was explored for GaAs in Section 3.9.5 where modes with frequencies smaller than the LO phonon were found. They may also show, at least qualitatively, the required decrease in frequency with increasing carrier concentration. Added complications arise in GaInAs from the additional phonon branch, but the equations are of essentially the same form as Eqn. 3.25-3.28 just with terms in $\omega_{\mathrm{LO}}$ replaced by two symmetric terms in $\omega_{\mathrm{L} 1}$ and $\omega_{\mathrm{L} 2}$.

For the simple coupled plasmon-LO phonon system the plasmon frequency has been calculated by Peeters et al. (1987), in the long wavelength limit, as:

$$
\omega_{\mathrm{p}}=\frac{\omega_{\mathrm{TO}_{1}}}{\omega_{\mathrm{LO}_{1}}} \frac{\omega_{\mathrm{TO}_{2}}}{\omega_{\mathrm{LO}_{2}}} \omega_{\mathrm{po}}
$$

where $\quad \omega_{\mathrm{po}}=\frac{2 \pi \mathrm{e}^{2} \mathrm{n}_{\mathrm{s}}}{\epsilon_{\infty} \mathrm{m}^{*}} \mathrm{k}$ is the unperturbed plasmon frequency.

As $n_{s}$ increases, for small $k$, there are three modes - at the GaAs-like and InAs-like LO frequencies and the plasmon - which all increase with $n_{s}$. The only mode of 
possible relevence moves from the InAs $L O$ at $n_{s}=0$ up to the GaAs $T O$ by $\mathrm{n}_{\mathrm{s}} \sim 10^{12} \mathrm{~cm}^{-2}$ and so clearly does not fit in with these results.

There also exists the possibility of intersubband scattering coupling to the electron-phonon plasma excitations. The coupled mode frequencies depend critically on the relative values of the inter-subband separations, $\hbar \omega_{01}$, and the optic phonon energies. When $\omega_{01}$ is small there is conventional plasmon coupling with modes above the LO and below the TO phonons. This situation corresponds to the wide QWs and heterojunctions. For $\omega_{01}>\omega_{L O}$ an intermediate mode exists which decreases in frequency from the LO to the TO value as $n_{s}$ increases. This would be the situation for the narrow wells (e.g. for $100 \AA$ a finite square well calculation gives $\omega_{01} \sim 740 \mathrm{~cm}^{-1}$ ), and may suggest a qualitative explanation for the behaviour shown in Fig. 4.3.

The results obtained by using values for the subband separation from finite square well calculations for $100 \AA$, $200 \AA$, and $300 \AA$ quantum wells in the long wavelength limit are shown in Fig. 4.14 for both (a) full two mode and (b) single mode behaviour. This figure indeed shows a decrease for the GaAs-like mode with increasing $n_{s}$, but only if $E_{01}$ lies above the phonon energies, as is the case in $100 \dot{A}$ and $200 \dot{A}$ wells, but not for wider wells or heterojunctions. Note that quantitatively correct values for the frequencies require calculations with $\mathbf{k}$ values appropriate to magnetophonon resonaces.

Objections to this picture are: (i) a decrease right down to the InAs-like TO at $226 \mathrm{~cm}^{-1}$ would only be possible if GaInAs exhibits single mode behaviour; (ii) different dependences would be predicted for heterojunctions and quantum wells and (iii) the rate of decrease of $\omega_{\text {LO }}$ is far too slow to quantitatively account for the experimental results unless $E_{01}$ is very close to $\omega_{\mathrm{LOI}}$.

Finally, if the magnetophonon resonace is due to coupling to these collective modes, why are they not see in cyclotron resonance measurements under the same conditions? By contrast in heavily doped bulk PbTe Ramage et al. (1977) find coupled mode behaviour in optical measurements, but the magnetophonon oscillations are firmly pinned at the frequency of the unscreened LO phonon. This was explained by the much larger wavevector associated with magnetophonon than optical processes. 
Figure 4.14 The change in frequency of LO phonon-intersubband plasmon coupled modes with carrier concentration asssuming (a) two mode behaviour and (b) single mode behaviour, which comes closer to reproducing the qualitative trends of Fig. 4.3. However the differences between samples must be recognised.
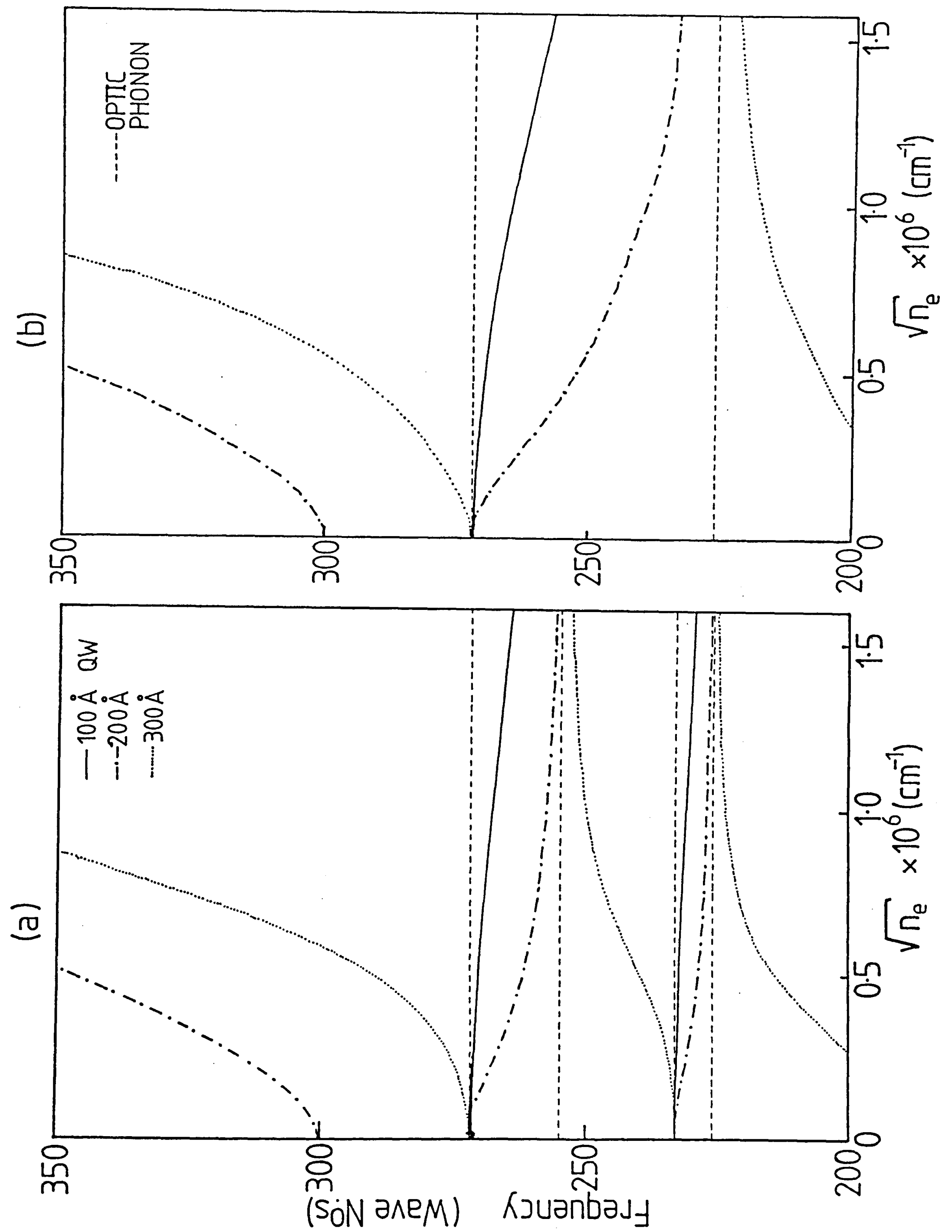


\subsection{SUMMARY}

Magnetophonon resonance has been studied in a variety of GaInAs-InP quantum wells and heterojunctions. The apparent frequency of the optic phonon coupling to the electrons of the $2 D E G$ decreased from $272 \mathrm{~cm}^{-1}$ to $229 \mathrm{~cm}^{-1}$, as the carrier density was increased regardless of the details of the particular sample. This is in stark contrast with the expected, fixed value of $272 \mathrm{~cm}^{-1}$ for the LO phonon, but is in qualitative agreement with carrier-concentration-dependent Raman measurements on similar samples and previous magnetophonon measurements on $2 \mathrm{D}$ GaInAs based systems.

Possible explainations for this striking change are explored, including: screening of the optic phonon electric field; interaction with coupled phonon-2D plasmon modes; and shifts in the resonance position due to a carrier concentration dependent broadening. A proper description of the problem may require the use of dynamic, k-dependant screening, and possibly also a full treatment of the alloy phonon modes including damping.

The amplitudes of the resonances in the narrow wells was found to increase with carrier concentration and so was the damping factor. In the heterojunctions the amplitudes were apparently random until plotted against mobility at $4 \mathrm{~K}$ where a strong correlation was found, reinforcing the results of the previous chapter on GaAs-GaAlAs.

On rotation in magnetic field, the amplitude of magnetophonon resonances from narrow quantum wells remained constant and their position depended on just the total magnetic field. By contrast the amplitude fell rapidly with angle in the heterojunctions and the resonance positions did not follow a pure $\cos \Theta$ dependence. There were exceptions to this rule which were explained in terms of subband energies.

The experimental results generally made more sense if the optic phonons in GaInAs were considered not to show full two mode behaviour, but consisted of a single Reststralen region between the GaAs-like LO at $272 \mathrm{~cm}^{-1}$ and InAs-like TO at $226 \mathrm{~cm}^{-1}$. 


\section{CHAPTER 5}

\section{HOT ELECTRON MAGNETOPHONON RESONANCE IN TWO DIMENSIONS}

5.1 Introduction

5.2 Hot Electron Energy Loss Mechanisms

5.2.1 Electron Temperature Model 144

5.2.2 Alternative Energy Loss Mechanisms Seen in Bulk 145

$\begin{array}{ll}\text { 5.2.3 Diffusion Picture } & 145\end{array}$

5.3 Hot Electron Magnetophonon Resonance in GaAs-GaAlAs Heterojunctions

5.3.1 Competition between HEMPR and Shubnikov-deHaas 146

$\begin{array}{ll}\text { 5.3.2 Experimental Considerations } & 147\end{array}$

$\begin{array}{ll}\text { 5.3.3 Electric Field Dependence } & 148\end{array}$

$\begin{array}{ll}\text { 5.3.4 Temperature Dependence } & 150\end{array}$

$\begin{array}{ll}\text { 5.3.5 Resonance Positions } & 151\end{array}$

5.4 Comparison with Theory

5.4.1 Explanation on the Basis of a Diffusion Picture 152

5.4.2 Explanation by Magneto-transport Calculation 154

5.4.3 Peak Inversion or Splitting? 156

5.6 Energy Loss by Resonant Inter-subband Scattering 160

$\begin{array}{lll}5.7 & \text { Summary } & 163\end{array}$ 


\section{$\underline{5.1 \quad \text { INTRODUCTION }}$}

The normal magnetophonon effect, discussed in the previous two chapters, arises from the resonant scattering of real optic phonons under ohmic conditions. This enhances the momentum relaxation at the resonance condition and gives a series of maxima in the resistivity. It was seen in Section 3.3.2 that at lower temperatures the optic phonon population is greatly reduced and the normal oscillations disappear. However, on application of an electric field parallel to the interface the electrons may be heated out of equilibrium with the lattice, becoming "hot electrons", and oscillatory structure returns to the magnetoresistance due to optic phonon emission. This hot electron magnetophonon resonance (HEMPR) has been observed in several bulk semiconductors (Nicholas 1985), but had not previously been directly observed in two-dimensional systems (Leadley et al. 1988b).

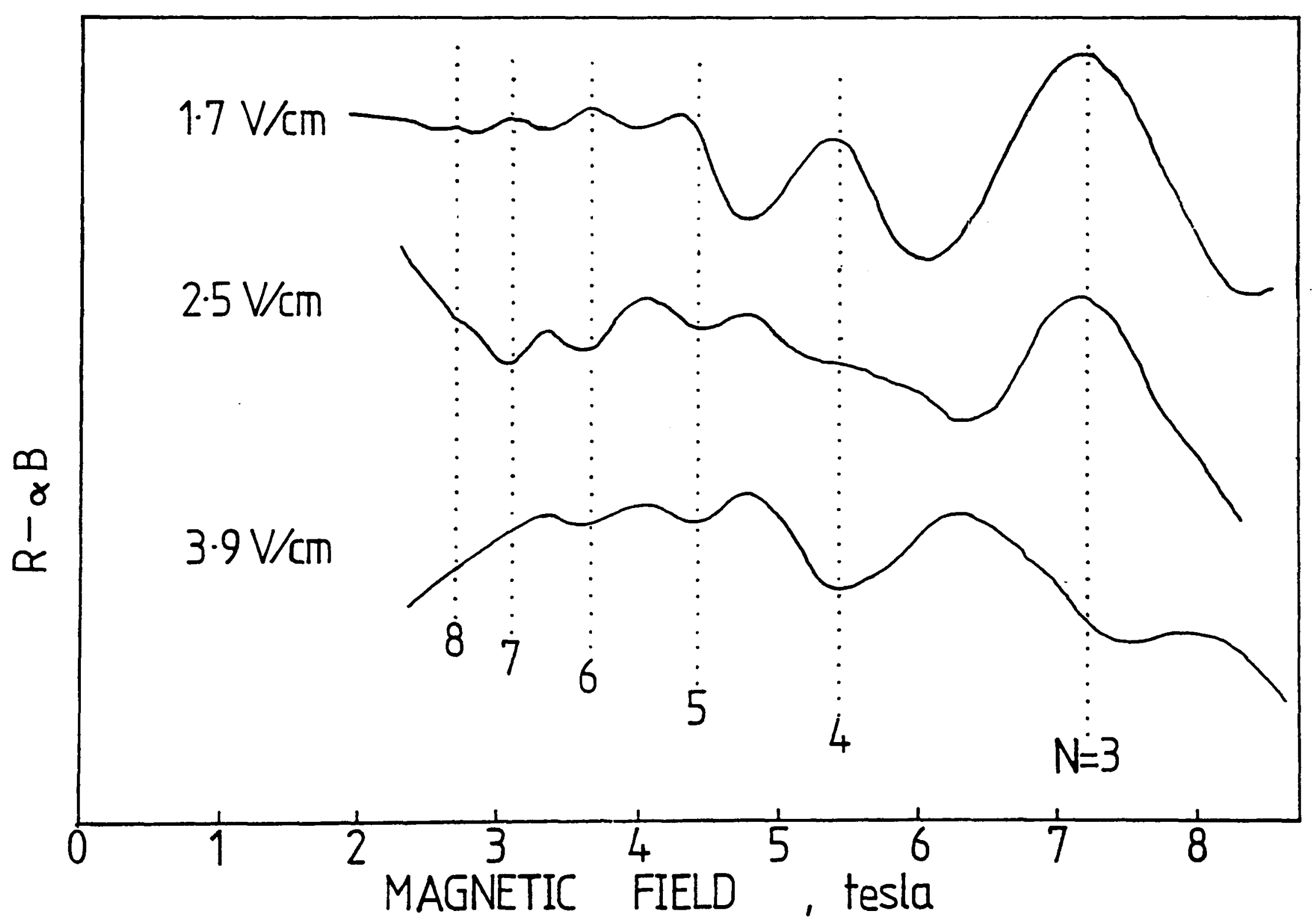

Figure 5.1 Linearly compensated traces in G139 showing the conversion of normal magnetophonon maxima into "hot electron" magnetophonon minima on increasing the electric field across the sample at $80 \mathrm{~K}$ [values of $E$ on the figure ]. Notice how the conversion occurs first at low magnetic fields. 
In this chapter direct observations of hot electron magnetophonon resonance extrema in the magnetoresistance are presented (Fig. 5.1) and some comparisons made with the available theory. The structure is especially interesting in the "warm" electron region at relatively high temperatures where the processes of phonon emission and absorption compete. A physical picture of electron flow through energy space will be considered to explain the observed behaviour in relation to the energy loss mechanisms. In Section 5.6 the occurrence of an additional series of oscillations will be attributed to the provision of an energy loss channel by resonant inter-subband scattering.

\subsection{HOT ELECTRON ENERGY LOSS MECHANISMS}

\subsubsection{Electron Temperature Model}

Under non-ohmic conditions resonant energy relaxation becomes more important than momentum relaxation in determining the structure of the magnetoresistance. As the energy relaxation rate (due to inelastic scattering) can be several orders of magnitude smaller than the carrier momentum relaxation rate (all types of scattering except elastic carrier-carrier scattering) it is possible to heat the electron system out of thermal equilibrium with the lattice with only a relatively small electric field. Then, as the carrier-carrier scattering is much faster than either of the previous two processes, an effective electron temperature, $T_{e}$, may be defined and the electronic distribution function consudered to be a Maxwellian distribution characterised by this temperature.

In this electron temperature picture the rate of energy loss will be largely determined by strong emission processes, eg. acoustic or optic phonons, from electrons in the high energy tail of the distribution. Any resonance in the phonon emission rates would then lead to a cooling of the electron system and it is this reduction of $T_{e}$ that is reflected in the transport properties. In heterostructures at low temperature the resistivity is dominated by impurity scattering and a reduction in temperature will lead to an increase in mobility. Hence there appear a series of minima in the resistivity at the magnetophonon resonance condition where a maximum in the optic phonon scattering rate occurs. 


\subsubsection{Alternative Energy Loss Mechanisms Seen in Bulk}

In bulk, much richer structure was found in the hot electron regime than for the normal effect, which is restricted to LO phonon scattering, and this can be understood by considering the above picture: any mechanism that is capable of giving a resonant path for energy relaxation will result in electron cooling and a series of oscillations in the resistivity. For example the emission of pairs of acoustic phonons was found to be extremely important by Stradling \& Wood (1970b). At the edge of the Brillouin zone there is a very high density of phonon states and in III-V materials the dispersion curve for TA phonons is very flat. There are therefore a large number of possibilities for emitting a pair of TA phonons with a comined wavevector near zero, as required for interaction with electrons. The proportion of electrons with sufficient energy to emit TA phonons will be very much larger than are able to emit LO phonons (at much higher energy) and so this becomes an important cooling mechanism. Series from both the $\mathrm{X}$ and the $\mathrm{L}$ points of the zone have been observed.

Another important process is associated with the emission of an LO phonon but rather than finishing in the conduction band the final electron state is associated with a shallow donor. This impurity capture process first observed by Stradling \& Wood (1968) occurs at an energy lower than inter-Landau level emission by the impurity binding energy, $E_{I}$, and so tends to be observed at lower temperatures.

\section{$\underline{5.2 .3 \quad \text { Diffusion Picture }}$}

Impurity capture has been seen to comletely dominate inter-Landau level LO phonon emission (Nicholas \& Stradling 1976) and this creates a problem within the electron temperature model, where both processes would be expected to be observed simultaneously. Further problems for an electron temperature picture arise from the gross distortions to the distribution function found in the presence of a strong resonant scattering mechanism. An alternative approach introduced by Yamada \& Kurosawa in 1973 considers electrons diffusing up in energy space under the influence of the electric field.

The diffusion process results from small changes in energy as the electron changes Landau state by acoustic phonon or impurity scattering. In such a process the 
conductivity is related to the real space diffusion coefficient, $D$, by the Einstein relationship:

$$
\sigma_{x x}=\frac{n e^{2}}{k T} \int_{0}^{\infty} D(E) f(E) g(E) d E=\frac{n e^{2}}{k T}<D>
$$

Diffusion continues until they reach a strong energy loss mechanism, whereupon electrons rapidly lose energy and return to the bottom of the conduction band. Above the threshold for emission the distribution function drops to zero within an excess energy of $k T$ [here $T$ is the lattice temperature].

Thus carriers reaching the threshold energy for impurity capture would never make it to inter-Landau level emission unless $k T>E_{\mathrm{I}}$, ie. the latter process is only seen at temperatures greater than the impurity binding energy. This is indeed seen and has been used to determine $\mathrm{E}_{\mathrm{I}}$ (Nicholas et al. (1979). At high temperature the relative importance of each process will be determined by the relevent density of final electron states. So for the capture process to completely dominate there must be sufficient available impurity states. In the modulation doped structures considered here this is not the case, which, together with the large density of states in each Landau level, makes impurity capture unlikely in 2D. However, the diffusion picture it has introduced is most useful for any strong or resonant scattering mechanism.

\subsection{HOT ELECTRON MPR IN GaAs-GaAlAs HETEROJUNCTIONS}

\subsubsection{Competition between HEMPR and Shubnikov-deHaas}

Observing HEMPR is not always straight forward: at high temperatures the normal resonances dominate; and at the lower temperatures where the HEMPR would be clear to see, most samples show strong Shubnikov-deHaas oscillations. These are often very pronounced at temperatures $\sim 80 \mathrm{~K}$ in GaAs-GaAlAs heterostructures and may even be observed right up to room temperature in materials of lighter effective mass, such as GaInAs-InP. However, in our samples the high mobilities at low carrier concentrations mean the Shubnikov-deHaas oscillations should be confined to fields well below the magnetophonon structure. For example, with a carrier concentration of $5 \times 10^{10} \mathrm{~cm}^{-2}$ the fundemental field of the Shubnikov-deHaas oscillations occurs at $1.0 \mathrm{~T}$. To observe any 
significant HEMPR structure in lower quality samples a higher carrier density would be required to give sufficient mobility, but then the resonances would become swamped by the Shubnikov-deHaas oscillations. This explains why direct measurements of HEMPR have not previously been reported for $2 \mathrm{D}$.

Inoue et al. (1983) observed an additional periodicity in the Fourier transform of the Shubnikov-deHaas oscillations at $4.2 \mathrm{~K}$ which increased with the electric field applied to the sample. This was interpreted as emission of optic phonons at the magnetophonon resonance condition. However, it appears that the electron temperature was below $10 \mathrm{~K}$ (which is similar to that found in Chapter 8) and it is difficult to see how the electrons could emit $296 \mathrm{~cm}^{-1}$ phonons of temperature $426 \mathrm{~K}$. Sakaki et al. (1984) also saw odd bumps on their Shubnikov-deHaas traces at the position of the $N=2 \& 4$ magnetophonon resonances, under warm electron conditions. However, they were only observed when the input power per electron exceeded $6 \times 10^{5} \mathrm{eV} / \mathrm{s}$, or $\mathrm{T}_{\mathrm{e}}>40 \mathrm{~K}$. By comparison Inoue et al. were claiming peaks in the Fourier spectrum with input powers two orders of magnitude lower. This may be due to the greater resolution gained by Fourier analysis or to the large carrier concentration in their sample of $1.4 \times 10^{12} \mathrm{~cm}^{-2}$, corresponding to a Fermi energy of $49 \mathrm{meV}$. This is already greater than the optic phonon energy without additional electron heating, but also means population of higher subbands from which other features could appear (see Chapter 6).

\subsubsection{Experimental Considerations}

The samples studied were the modulation doped GaAs/GaAlAs heterojunctions described in Section 2.8. Most of the quantitative data reported here is from the sample of lowest electron concentration, G139. The hot electron magnetophonon resonances also lead to small changes in the resistivity, and so the oscillations were enhanced by either taking a second derivative or compensating the signal. The measurements all used a constant current through the sample and by increasing this higher electric fields were applied along the 2DEG. In the range of measurements, up to $10 \mathrm{~V} / \mathrm{cm}$, the mobility both at zero field and $9 \mathrm{~T}$ was found to remain constant, which shows no lattice heating occured. At the lower temperatures the low field 
resistivity showed a much stronger temperature dependence than that in high field, eg. In $\mathrm{G} 139$ at $60 \mathrm{~K} \mu(0)=17.5 \mathrm{~V}^{-1} \mathrm{~s}^{-1} \mathrm{~m}^{2}$ and near the $\mathrm{N}=4$ resonance $\mu(6 \mathrm{~T})=1.4 \mathrm{~V}^{-1} \mathrm{~s}^{-1} \mathrm{~m}^{2}$. Because of this strong field dependence the ratio $\Delta \rho / \rho(B)$ was used as a measure of the resonance amplitude, where $\rho(B)$ is the resistivity at the resonance field, (cf. Section 3.3.1) and the electric field was also evaluated at the resonance field. The largest electric field for which reproducible results were obtained was $10 \mathrm{~V} / \mathrm{cm}$ which corresponds to an input power of $1700 \mathrm{eV} / \mathrm{s}$ per electron at $60 \mathrm{~K}$. The limit on electric field was imposed by the contacts which tended to break down at high current densities.

\subsubsection{Electric Field Dependence}

Figure 5.1 shows compensated traces of the magnetoresistance at $80 \mathrm{~K}$. At this temperature there are still some normal resonances, but as the electric field is increased the resistance extrema invert, starting at low magnetic fields [high harmonic number] to become HEMPR minima at the same resonance positions (Eqn. 2.17). These can become strong oscillations and large fractions of the resistivity, as shown in Fig. 5.2.

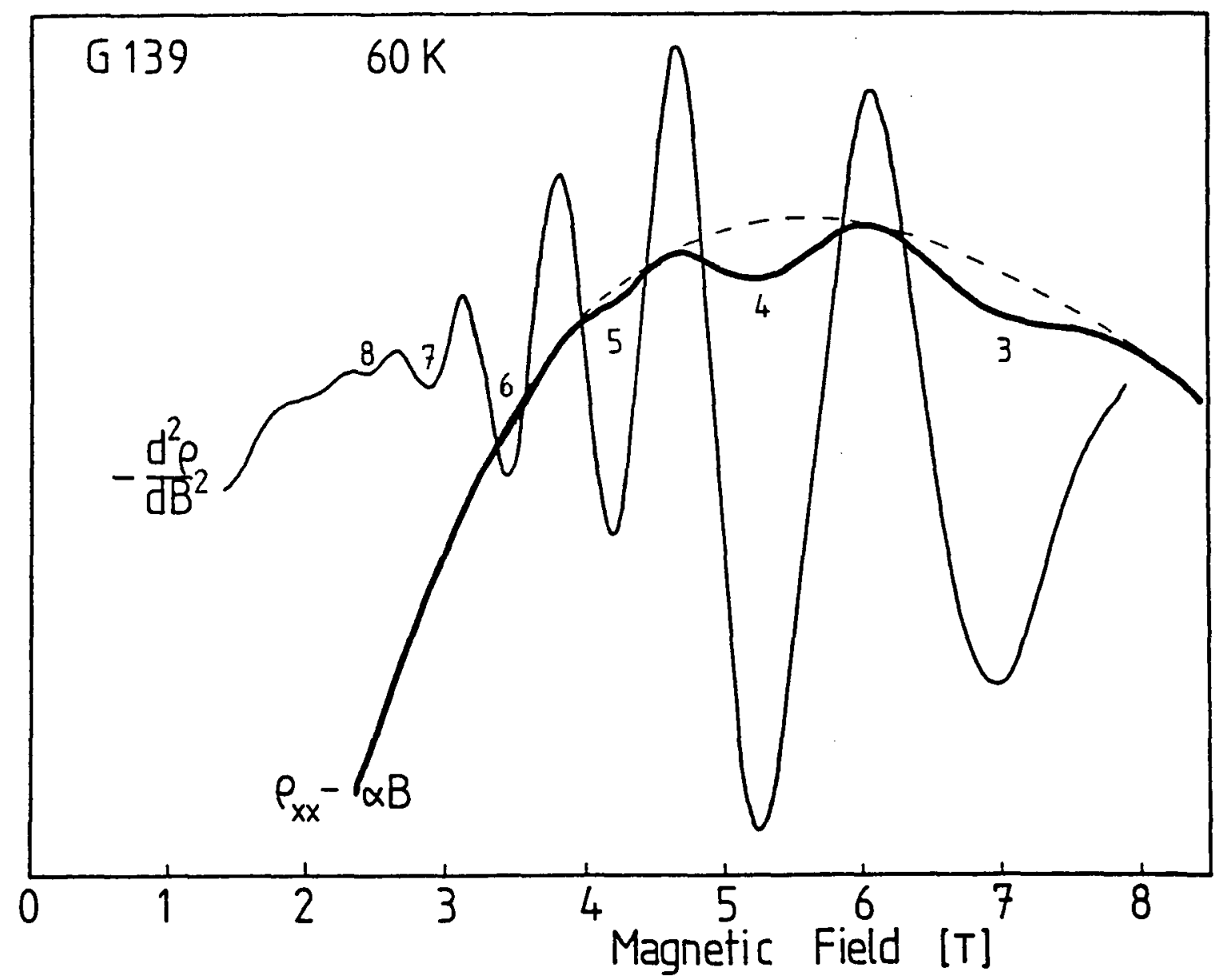

Figure 5.2 Compensated and double differentiated recordings of HEMPR at its maximum intensity, showing very strong minima in the resistivity. An indication of the unperturbed resistivity is shown by the dashed line. 
On increasing the electric field further the amplitude of the oscillations increases rapidly as shown in Fig. 5.3. The amplitude varies with the electric field as approximately $E^{2}$. This is as expected since the power input per electron is given by $\mathrm{e} \mu \mathrm{E}^{2}$ and if LO phonon emission is the dominant energy loss mechanism the resonance amplitude will be a measure of the energy relaxation rate.

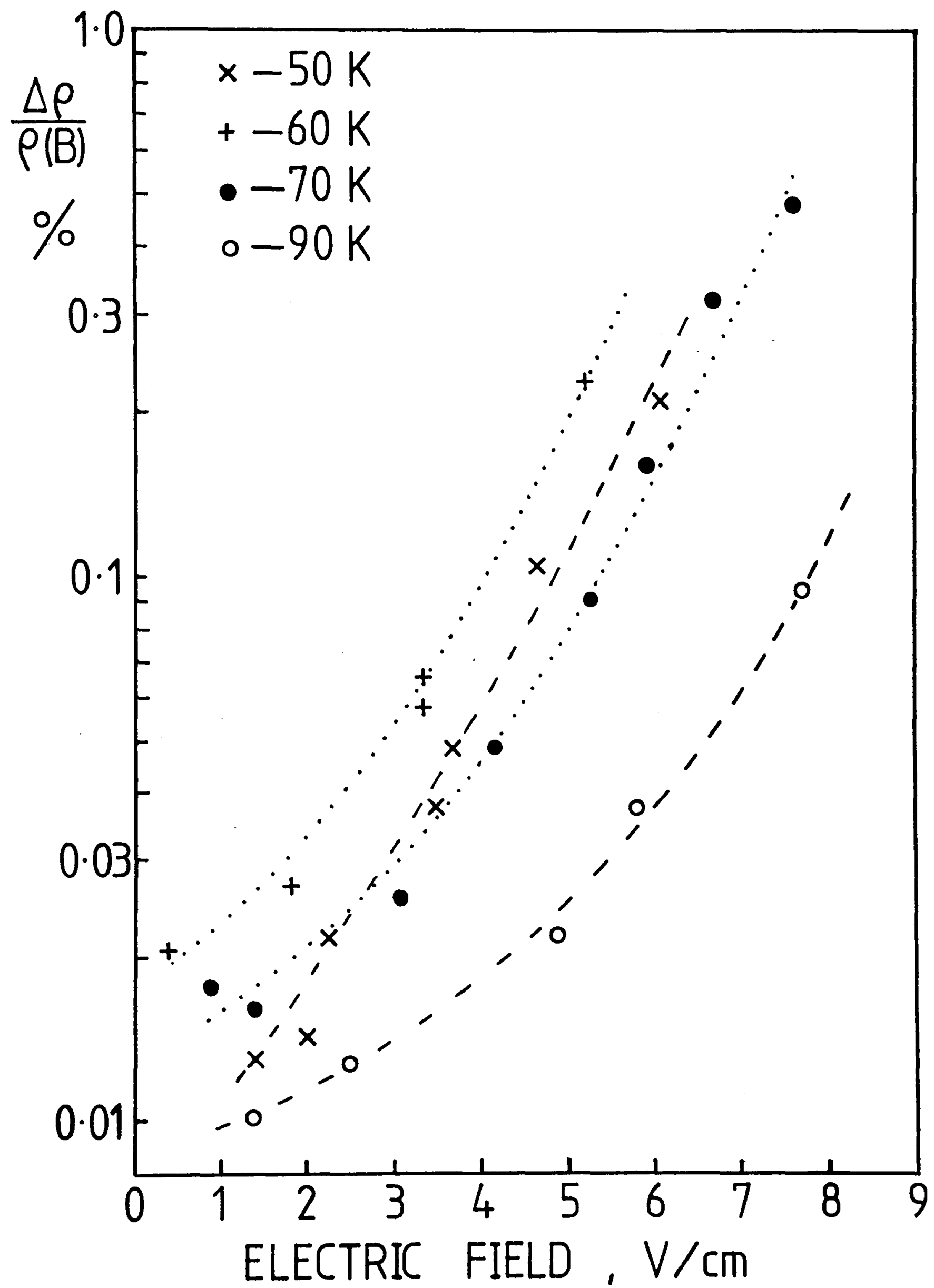

Figure 5.3 Amplitude of the $\mathrm{N}=5 \mathrm{HEMPR}$ minimum, normalised to the resistivity at the resonance field, shown increasing rapidly with electric field in G139 at: temperatures between $50 \mathrm{~K}$ and $90 \mathrm{~K}$ (as indicated on the figure). 


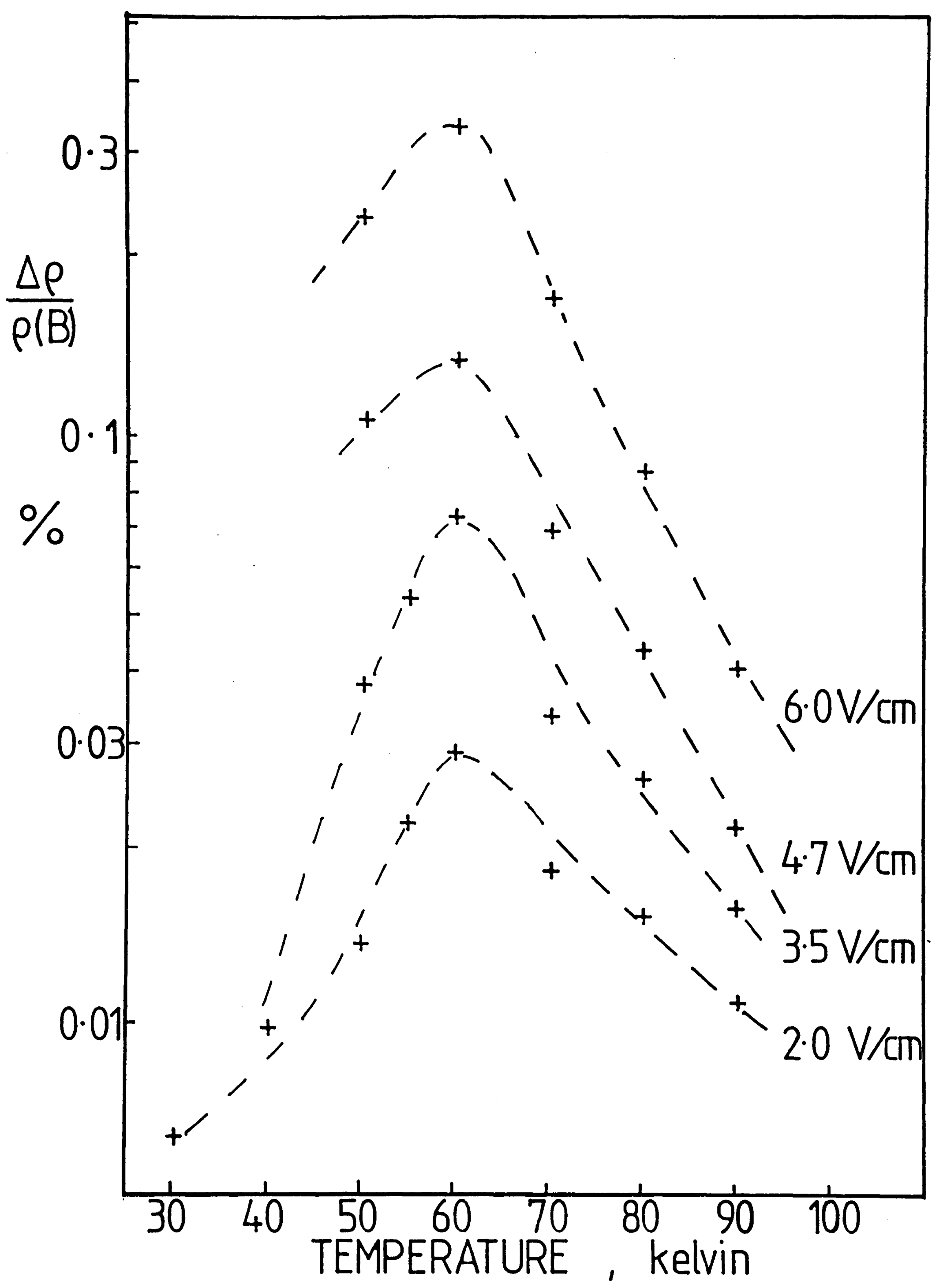

Figure 5.4 Normalised amplitude of the $N=5$ hot electron magnetophonon minima in G139 shown as a function of temperature at several values of electric field, demonstrating an obvious maximum intensity at $\sim 60 \mathrm{~K}$. 


\subsubsection{Temperature Dependence}

The hot electron magnetophonon resonances are observed for lattice temperatures between 30 and $100 \mathrm{~K}$ with a maximum in amplitude at around $60 \mathrm{~K}$, as shown in Fig. 5.4 for the $N=5$ resonance. [Note that the electron temperatures, if such can be defined, may be significantly higher than this due to the large current densities used.] By contrast, for a shifted Maxwellian distribution, a monotonic increase in the strength of the oscillations would be expected on cooling, since at lower temperatures the mobility increases and for a given electric field the power input per electron would be larger. A lower limit for observing the resonances would also not be predicted as the electrons could always be heated to the phonon energy. However, the value of the lower limit is in agreement with the number produced by Sakaki et al. (1984) and the conclusions of Shah et al. (1983), who established from a study of phototluminescence that optic phonon emission is the dominant energy loss mechanism for electrons with $\mathrm{T}_{\mathrm{e}}>40 \mathrm{~K}$. The decrease in amplitude at low temperatures is however in contrast to the HEMPR reported by Inoue et al. (1983) from the $4.2 \mathrm{~K}$ magnetoresistance.

It may be significant for the position of the maximum that the high field mobility is always phonon limited in the temperature range of interest and that $\approx 60 \mathrm{~K}$ is the temperature at which the mobility (Foxon et al. 1986) and the cyclotron resonance linewidth (Hopkins et al. 1987) change from being dominated by acoustic phonon to optic phonon scattering.

\subsubsection{Resonance Positions}

By using Eqn. 2.17 and the effective mass measured by cyclotron resonance at the required temperature the frequency at which the electron-optic phonon coupling occurs can be calculated. The fundamental field deduced from these magnetophonon measurements has been corrected for damping using Eqn. 3.16 and the effective mass corrected for non-parabolicity and resonant polaron effects as described in Section 3.4. In perpendicular fields this gives a value of $280 \pm 2 \mathrm{~cm}^{-1}$ at $50 \mathrm{~K}$ for all the samples studied, well below the frequency of the GaAs LO phonon $\left(296 \mathrm{~cm}^{-1}\right)$ normally assumed to dominate the scattering and in agreement with the work of Chapter 3 on 
the normal magnetophonon resonances.

The effect of rotating the sample relative to the magnetic field can be seen in Fig. 5.5, which shows that the coupling energy increases towards the LO phonon energy at high angles. The rotation also causes the amplitude to decrease rapidly, falling by a factor of two only $20^{\circ}$ away from perpendicular. This behaviour is again similar to that seen in the normal resonances.

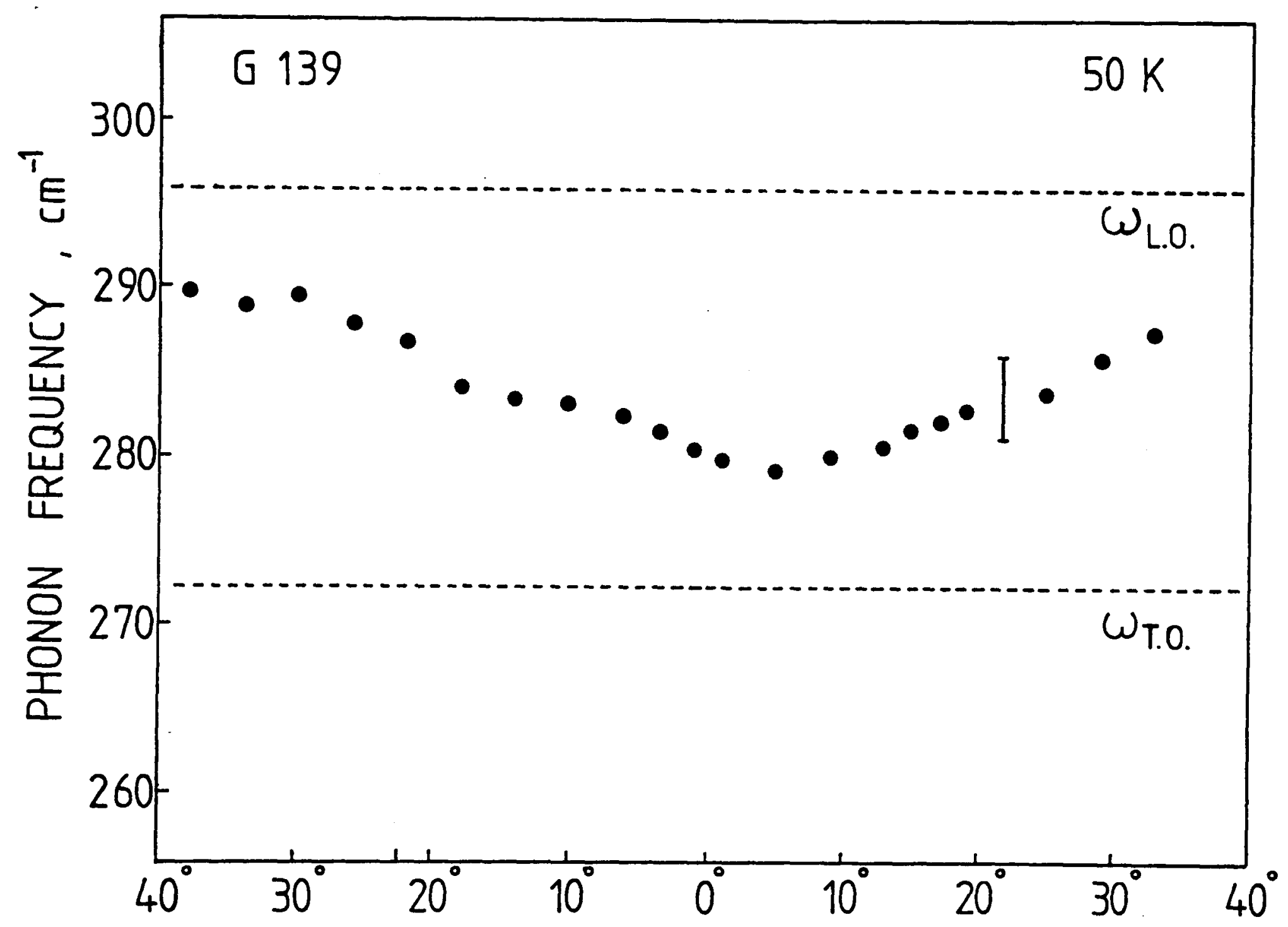

Figure 5.5 Orientational dependence of the coupling frequency deduced from the HEMPR resonace position, which is well below the bulk LO frequency and shows a very similar trend to the normal resonances of Fig. 3.16.

\section{$\underline{5.4 \text { COMPARISON WITH THEORY }}$}

\subsubsection{Explanation on the Basis of a Diffusion Picture}

The fall in amplitude below $60 \mathrm{~K}$ is believed to be due to the complete quantization of the density of states in a 2-D system. As the temperature is reduced the Landau levels become extremely sharp (Hopkins et al. 1987), leading to very low values of the density of states between the levels (depicted schematically in Fig. 5.6ii). This is clearly observed in studies of the cyclotron resonance mass where Brummell et al. (1987a) find 
that occupation effects arising from this sharp quantization become important below $\sim 100 \mathrm{~K}$. This means that at low temperature there are very few states in the Landau gap which are sufficiently close in energy to allow electrons to move up in energy space by the low energy scattering processes required for diffusive transport. Therefore the number reaching higher Landau levels and being capable of emitting optic phonons is greatly reduced. At higher temperatures there are more extended states between the levels (Fig. 5.6iii) and so more electrons can be rapidly accelerated to higher Landau levels and reach the optic phonon energy giving larger resonance amplitudes. The fall at higher temperatures just comes from competition between normal and hot electron effects.

Cyclotron resonance also shows that the level broadening varies slowly over the field range of interest (Hopkins et al. 1986) and so from Figs. 5.6 (i) and (ii) it can be seen that for transition of high harmonic number, at lower magnetic fields, there will be more overlap of Landau levels providing an easier path to an energy of $\hbar \omega_{\text {LO }}$. Thus these high $\mathrm{N}$ resonances should have a greater amplitude, and show hot electron minima sooner, than those of low $\mathrm{N}$, which is indeed the case observed in Fig. 5.1.

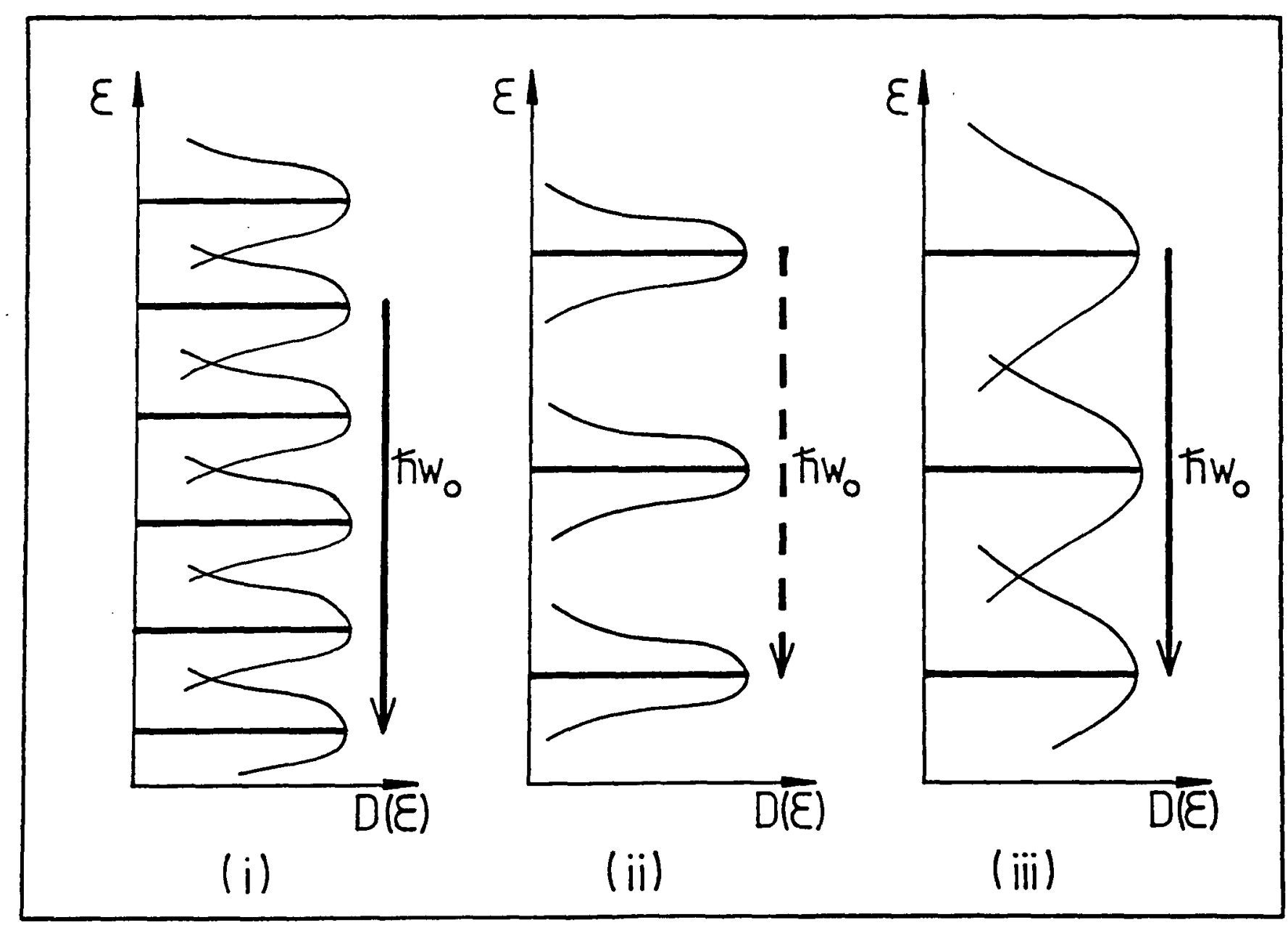

Figure 5.6 A schematic view of the density of states for: (i) an $N=4$ resonance; (ii) an $\mathrm{N}=2$ resonance and (iii) $\mathrm{N}=2$ at higher temperature. 


\subsubsection{Explanation by Magneto-transport Calculation}

Use of the energy and momentum balance equation was outline in Section 2.4 and this has been extended into the non-linear regime by Warmenbol et al. (1988). This produces additional terms into the expression for resistivity - Eqn. 2.26 - which depend strongly on the level broadening and the percentage of flat background density of states included for both their magnitude and sign. However, closed form analytic expressions are only found for zero background and so the equations must usually be solved numerically.

In all their work Warmenbol et al. use the average electron velocity $v / v_{L O}$ as a parameter describing how "hot" the electrons are, where $v_{L O}=\left(2 \hbar \omega_{L} / m^{*}\right)^{\frac{1}{2}}$, whereas experimentally it is the electric field that is measured. One could the define $E_{\mathrm{LO}}=\mathrm{v}_{\mathrm{LO}} / \mu$, but would then need to decide whether to use the mobility in field, giving $E_{\mathrm{LO}}=3300 \mathrm{~V} / \mathrm{cm}$, or the zero field value giving $250 \mathrm{~V} / \mathrm{cm}$. Depending on the broadening used they find changes from the normal magnetophonon structure occuring in the resistivity near the fundemental resonance for $\mathrm{v} / \mathrm{v}_{\mathrm{LO}}>0.05$ to 0.1 , and at lower velocities for the higher harmonics. Taking the maximum field used of $10 \mathrm{~V} / \mathrm{cm}$ and $E_{\mathrm{LO}}=250 \mathrm{~V} / \mathrm{cm}$ means $\mathrm{v} / \mathrm{v}_{\mathrm{LO}}$ is always less than 0.04 , so although this theory may provide some qualitative explainations of the phenomena, a quantitative description will require more detailed modelling of the actual physical system. Further it should be remembered that the numerical results are fairly sensetive to the form of density of states assumed.

Using $\Gamma_{0}=3.7 \mathrm{meV}$, which compares with an experimental damping factor $\tilde{\gamma}=1.3$ for the $\mathrm{N}=5$ resonance [cf. Eqn. $2.20 \& 3.9$ ], Warmenbol et al. (1988) find that as the average electron velocity (electric field) is increased the magnetophonon extrema change from maxima to minima - Fig. 5.7. This is seen to occur first at low magnetic field, in accord with the experimental results of Fig. 5.1. [The agreement is more remarkable when it is considered that the calculations and measurements were made concurrently without knowlage of the other!] 


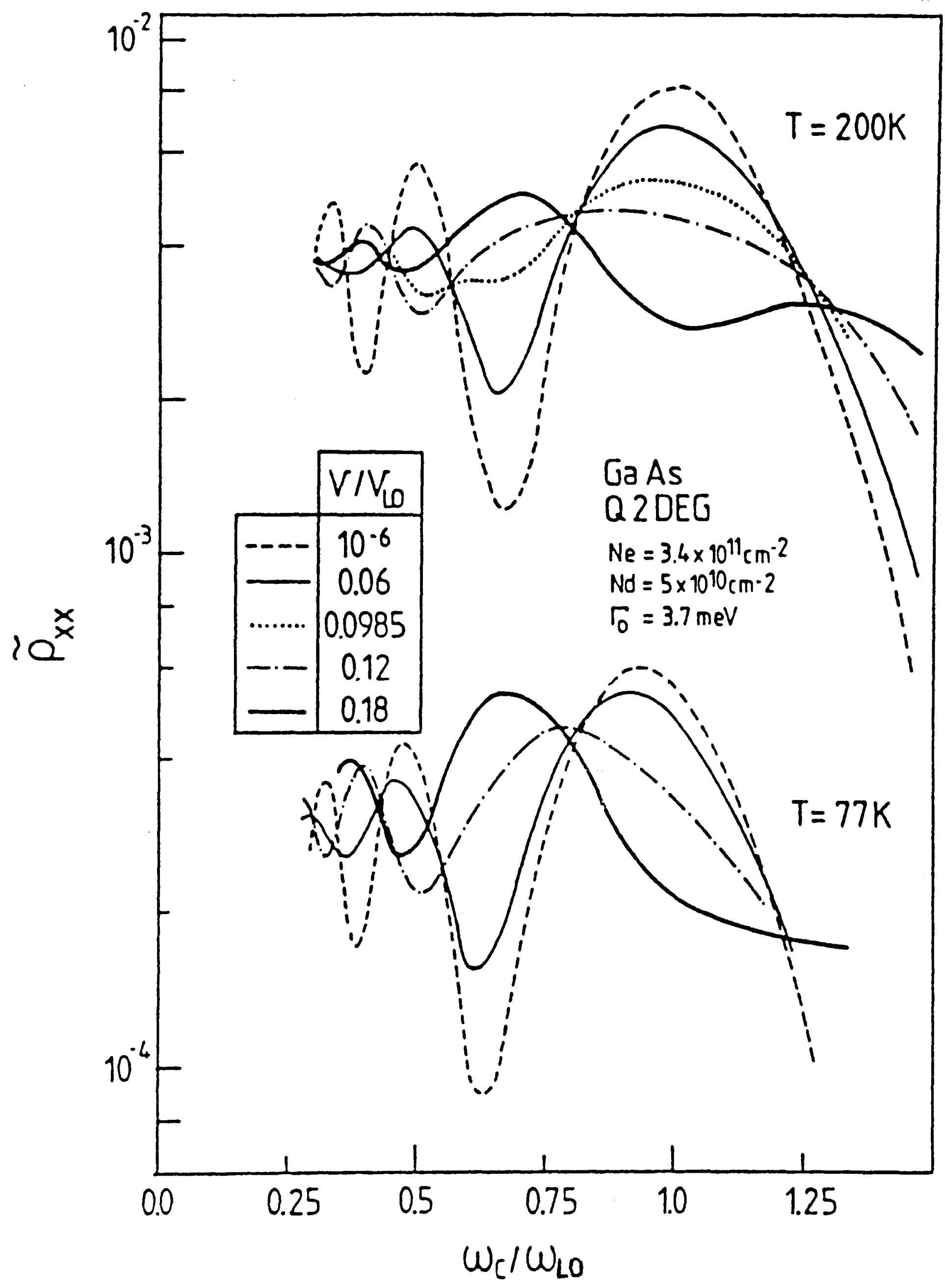

Figure 5.7 Calculation of the non-linear magnetoresistance by Warmenbol et al. for Gaussian broadened levels of width $\Gamma_{0}=3.7 \mathrm{meV}$, (comperable to the experimental situation), showing how the magnetophonon maxima change to HEMPR minima on increasing the average electron velocity $\left(v / v_{L O}\right)$. As in the experimental case the extrema at low field invert first. 


\subsubsection{Peak Inversion or Splitting?}

Warmenbol et al. (1989) then investigated small broadenings and with $\Gamma_{\mathrm{o}}=0.4 \mathrm{meV}$ (corresponding to $\tilde{\gamma}=0.4$ at $\mathrm{N}=5$ ) found the magnetophonon peaks split into two peaks, labled $\mathrm{N}^{+}$and $\mathrm{N}^{-}$, which shifted in magnetic field in opposite directions as the average electron velocity was increased - Fig. 5.8. At sufficiently high electric field the $\mathrm{N}^{-}$peak was found to merge with the $(\mathrm{N}+1)^{+}$peak at the $\mathrm{N}+\frac{1}{2}$ position, to give a series of minima at the magnetophonon condition, as found before with large $\Gamma_{0}$.

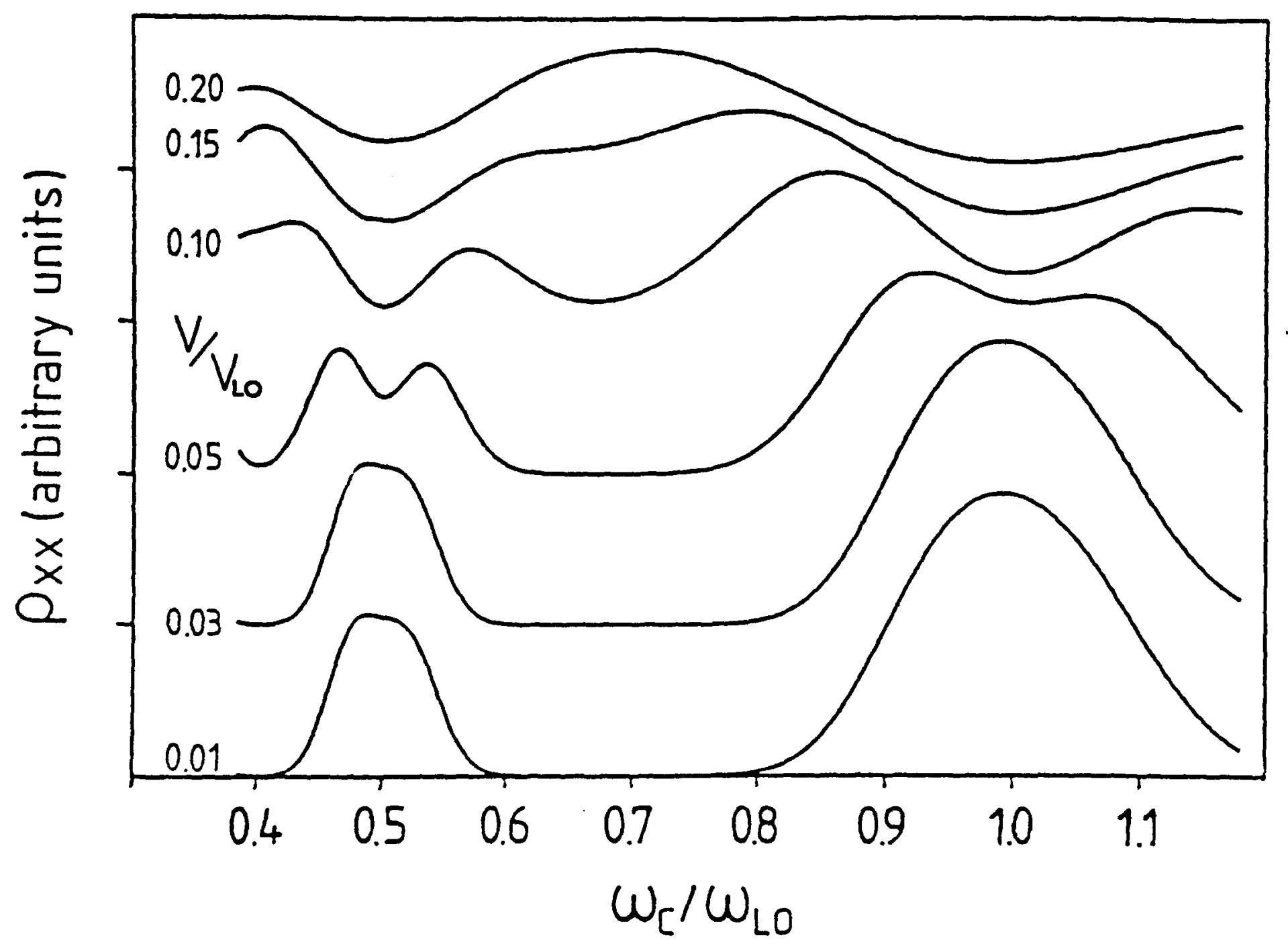

Figure 5.8 Simulation of the non-linear resistivity by Warmenbol et al. at $140 \mathrm{~K}$ with $\Gamma_{0}=0.4 \mathrm{meV}$, showing how the $N=1 \& 2$ magnetophonon maxima split and shift to form HEMPR minima as the electron velocity increases.

The force term $F(v)$ may be written to show the contribution from LO phonon absorption and emission explicitly, by making the transformation $E \rightarrow E^{\prime}=E+\hbar \omega_{L} O^{-\hbar q . v}$ The integral in Eqn. 2.25 in energy space becomes:

$$
\begin{aligned}
I_{E}=\int_{-\infty}^{\infty} d E & \exp (-E / k T) \operatorname{Im}\left[G_{m}(E)\right] \\
& \times\left[n\left(\omega_{L O}\right) \operatorname{Im}\left[G_{n}\left(E+\hbar \omega_{L O}-\hbar q \cdot v\right)\right]-\left[1+n\left(\omega_{L O}\right)\right] \operatorname{Im}\left[G_{n}\left(E-\hbar \omega_{L O}+\hbar q \cdot v\right)\right]\right]
\end{aligned}
$$

where $G_{n}(E)$ is the electron Greens function for Landau level n. The first term of 
Eqn. 5.2 represents absorption and the second emission. The shifts of these terms were analysed seperately resulting in peak positions given by:

$$
\omega_{c}=\frac{\omega_{L O}}{N} \pm \frac{q_{L O}}{N}
$$

for small velocities and zero broadening. The results of the full calculation for the $1^{ \pm}$ and $2 \pm$ peaks are shown in Fig. 5.9 from which the conversion from maxima to minima is apparent. The down shifted peak was associated with LO phonon absorption and the up shifted peak with emission.

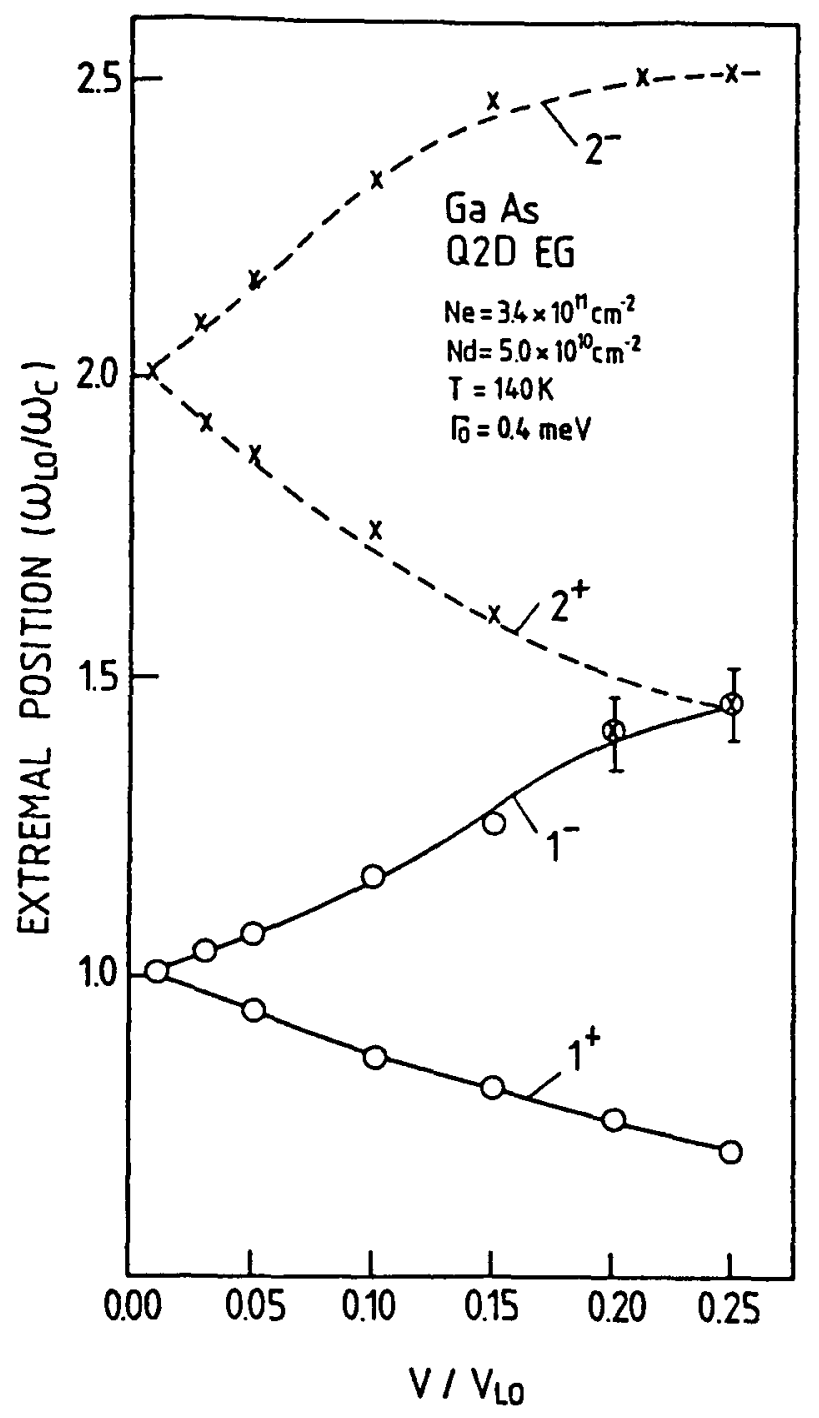

Figure 5.9 Calculated positions of the $N=1 \& 2$ maxima in units of $\omega_{L} d \omega_{c}$ as a function of average electron velocity for $\Gamma_{0}=0.4 \mathrm{meV}$ at $140 \mathrm{~K}$.

Experimentally the transition from maxima to minima is illustrated in Fig. 5.1 and in more detail in Fig. 5.10 where it can be seen that the high field peaks are normal maxima but at low field there are HEMPR minima. Between these two regions the $N=5$ resonance is undergoing a transition, but it is difficult to say whether it is splitting into $5^{+}$and $5^{-}$peaks, or a competing minimum is forming in the centre of the magnetophonon maximum. In support of the picture of Warmenbol et al. the down 
Figure 5.10 Double differentiated trace of magnetoresistance at $90 \mathrm{~K}$ in $\mathrm{G} 139$ showing distinct regions of normal magnetophonon maxima ( $=3 \& 4)$ and hot electron magnetophonon minima $(N \geqslant 6)$. There is a transition region at $\mathrm{N}=5$, which might be interpreted as a splitting into $5^{+}$and $5^{-}$peaks.

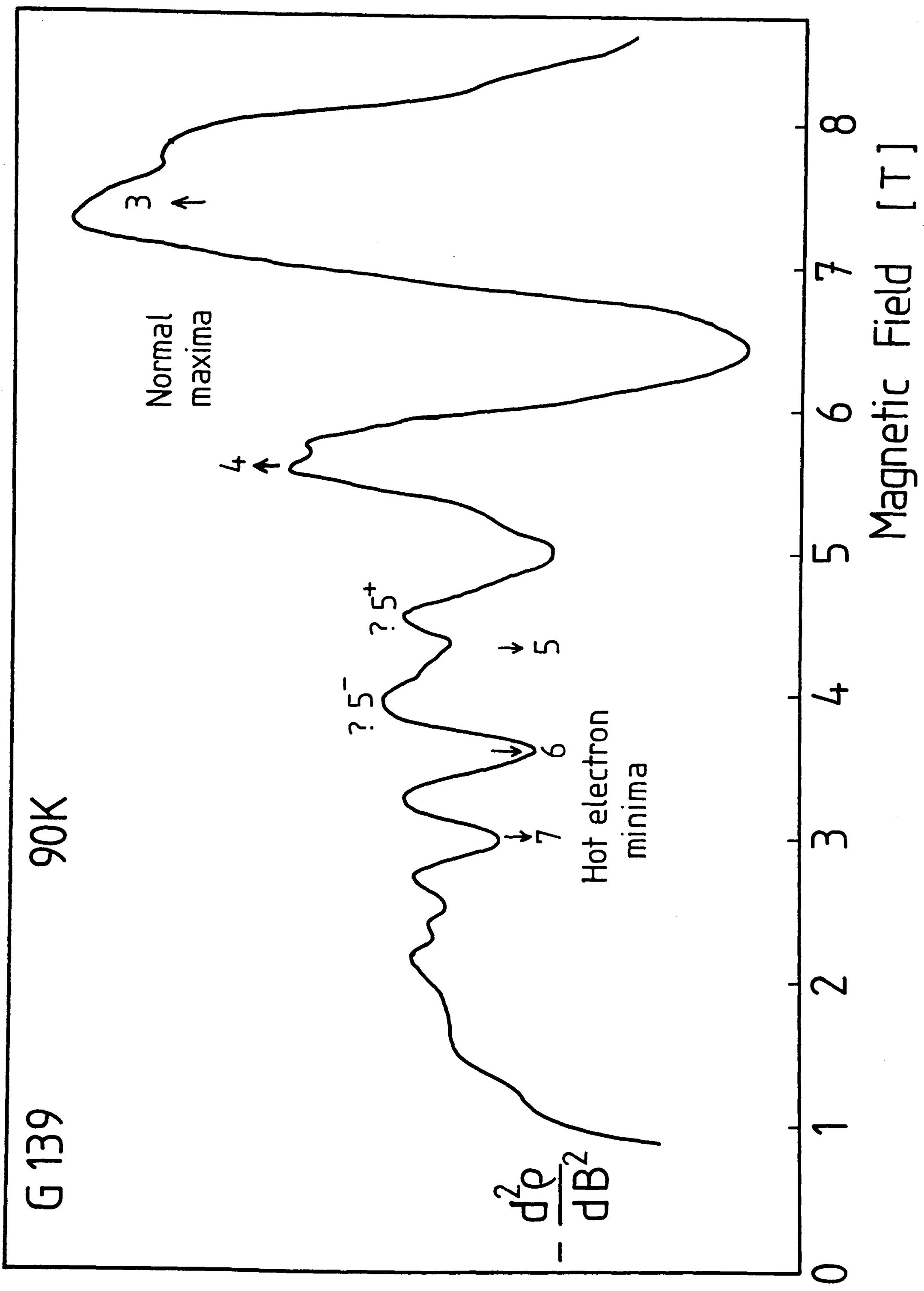


shifted peak associated with phonon absorption always appears stronger than the up shifted peak. But, according to the same theory, minima should only appear when peaks shifting from adjacent maxima merge, whereas in Fig. 5.10 the $\mathrm{N}=6$ minima is well formed while $\mathbf{N}=5$ is still splitting.

The transition might be clearer on a direct trace, rather than a differentiated trace where the peak shapes may be distorted, but on direct traces the maxima just vanish and reappear as minima at higher electric fields. This is in general agreement with the above theory, which only predicts observation of splitting in direct traces for small $\Gamma_{0}$. The low value of $\Gamma_{0}$ taken by Warmenbol et al. is from cyclotron resonance linewidths, which will be much smaller than observed in magnetophonon experiments at the same temperature, because the latter measurements are all made at the resonant polaron condition where the levels will be broadened. In fact cyclotron resonance linewidths do increase rapidly towards the polaron region, but this is not where the experiment is usually performed.

If the peaks don't split then Warmenbol et al. (1989) claim they must all shift to lower fields and form a series of maxima at half integral harmonics. One good test would be observation of the fundemental resonance in a direct trace, at higher magnetic fields than have been used to date. If it splits the $1^{+}$peak should move up in magnetic field on increasing the electric field and be clearly observable as there will be no corresponding $0^{-}$peak. Otherwise it would be predicted to shift to lower fields.

There remains an alternative explaination: the HEMPR minima actaully appear at the same field as the normal maxima and the resulting resistivity is a convolution of the two independent but competing effects. This picture is supported by Fig. 5.2 where the direct traces appears to show a real series of minima (rather than maxima) superimosed on the background, which is shown dashed. Also in Fig. 5.10 embryonic minima can be seen eating their way through the $N=3 \& 4$ maxima as well as the well developed specimen at $\mathbf{N}=5$. Physically this means that the resonant energy relaxation initially only occurs close to the resonance condition, whereas off resonance there is still a fairly large contribution from enhanced momentum relaxation. 


\subsection{ENERGY LOSS BY RESONANT INTER-SUBBAND SCATTERING}

In samples $G 83$ and $G 71$, with carrier concentrations of $\approx 2 \times 10^{11} \mathrm{~cm}^{-2}$, additional minima to those associated with magnetophonon resonance appear, shown by the arrows in Fig. 5.11. At low electric fields these are much stronger than the hot electron magnetophonon resonance, and appear to be periodic in $1 / \mathrm{B}$ with a fundamental field $\mathrm{NB}_{\mathrm{N}}$ of $10.8 \mathrm{~T}$. Similar additional structure is seen in the magnetoresistance of G139 after increasing the electron concentration to $5 \times 10^{10} \mathrm{~cm}^{-2}$ by persistent photoconductivity. Fig. 5.12 shows that at low electric field there is a series of minima with a fundamental field of $8.8 \mathrm{~T}$. On increasing the electric field the magnetophonon minima again dominates the resistivity.

These extra series may be due to the emission of pairs of zone edge acoustic phonons seen in bulk as mentioned earlier. However, the most likely candidate in GaAs is the $T A(X)$ phonon which has a frequency of $82 \mathrm{~cm}^{-1}$ (Dolling \& Waugh 1965), giving a fundamental field of $12.4 \mathrm{~T}$ in $\mathrm{G} 139$ and $0.2 \mathrm{~T}$ greater in the other samples. This is clearly at variance with our observations. Further complications arise in that to conserve momentum one of the phonons would have to be emitted across the interface, which would give higher fundamental fields as the $T A(X)$ frequency is higher in GaAlAs.

An alternative explanation is that these minima are due to an inter-subband resonance, depicted schematically in Fig. 5.13. When the Landau levels in the two subbands are coincident electrons can be scattered elastically between subbands whenever:-

$$
E_{10}=N \hbar \omega_{c}=N \hbar e B / m^{*} \quad N=1,2,3 \ldots
$$

where $E_{10}$ is the energy difference between the subbands.

Initially it may be considered that the additional scattering should give an increase in resistivity. However, this inter-subband scattering, as well as favouring transitions towards an equilibrium state, requires the emission of an acoustic phonon for wavevector conservation, and so will allow the electron system to cool. When the resonance 
Figure 5.11 Second derivative of the magnetoresistance of G83 at 60K. The dashed lines show the expected positions of HEMPR and the arrows indicate an additional series.

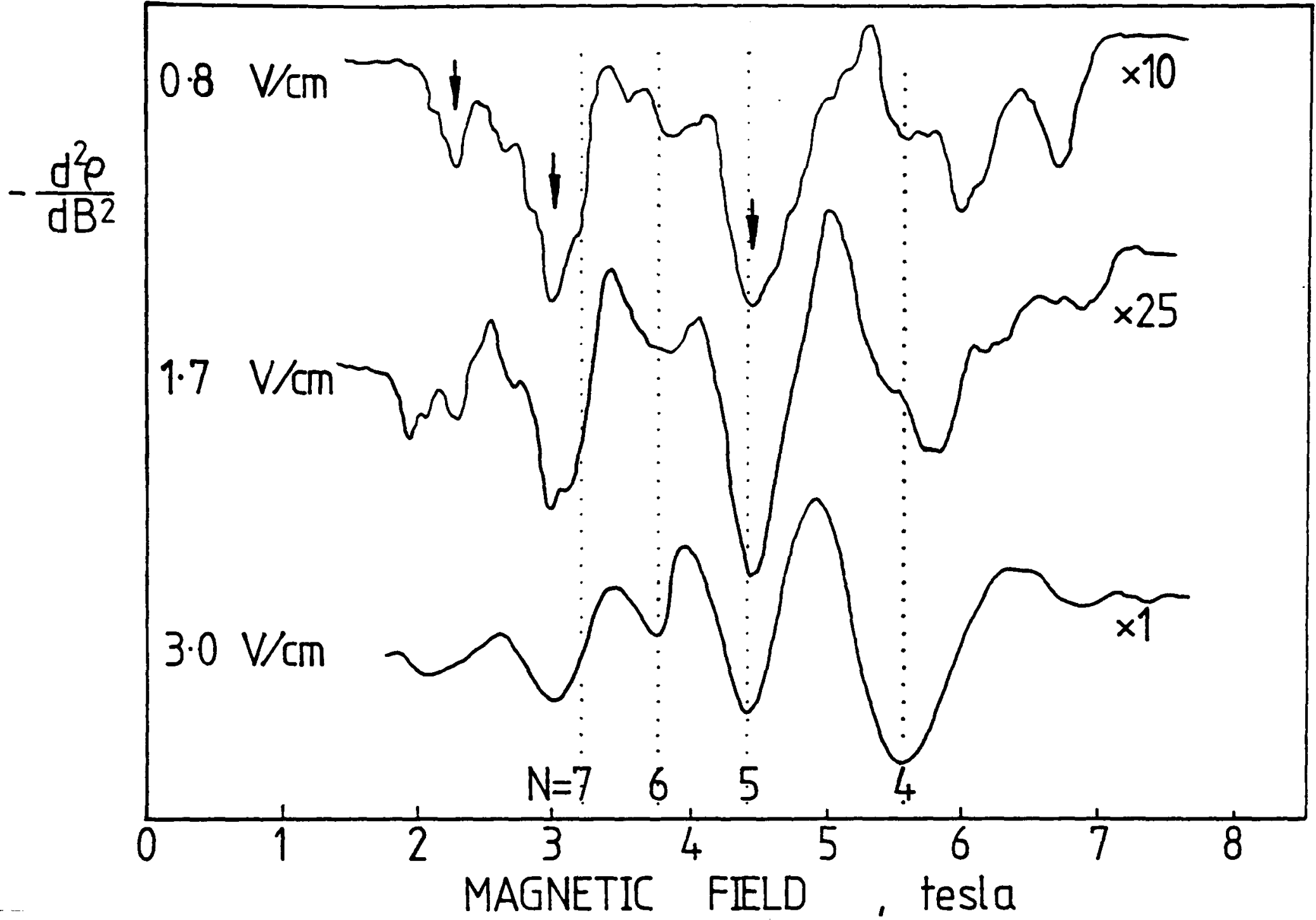

Figure 5.12 Hot electron magnetophonon resonance in $\mathrm{G} 139$, after illumination, at $70 \mathrm{~K}$ showing additional peaks at low electric fields. At higher electric fields the HEMPR again dominates.

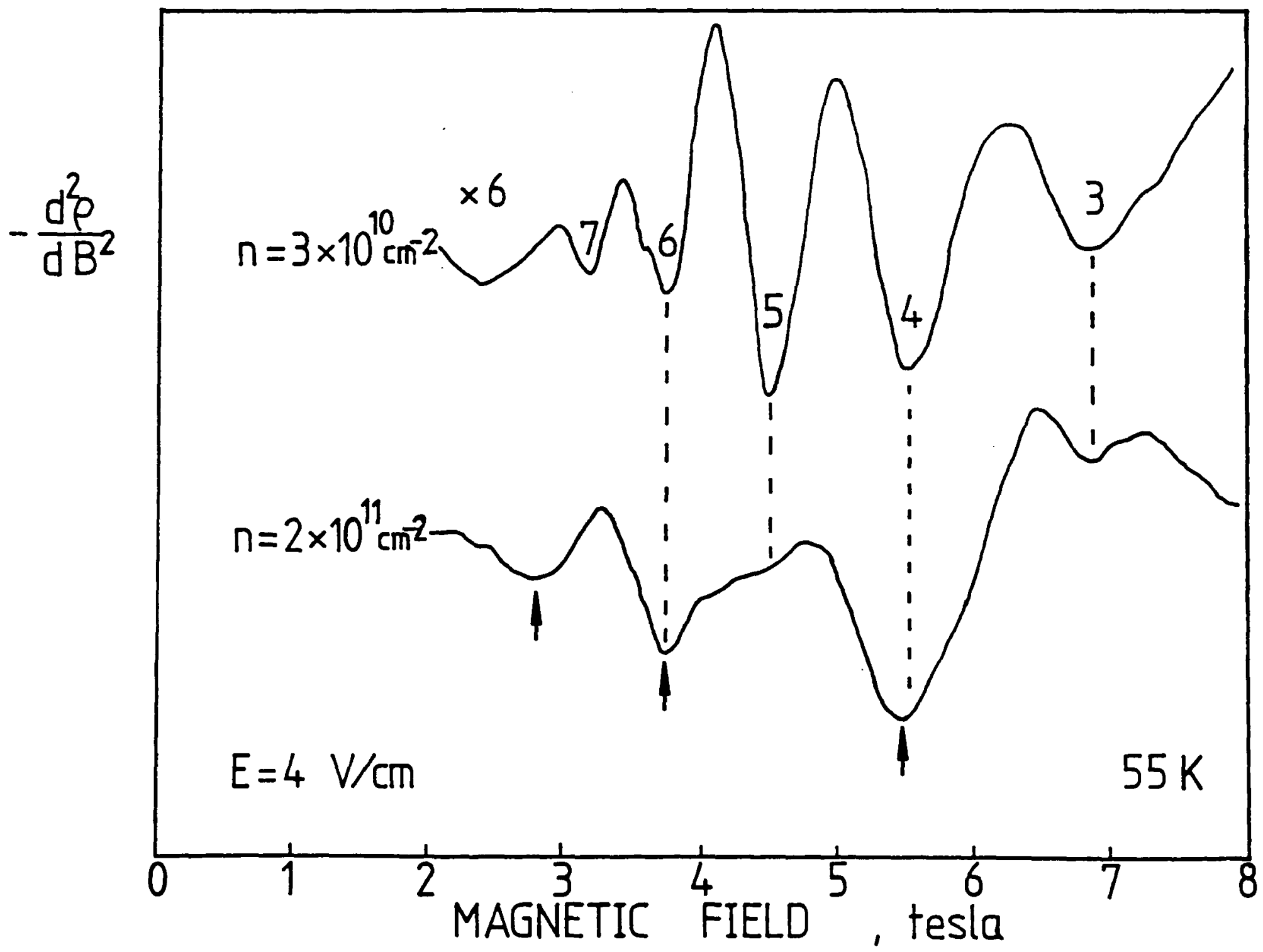




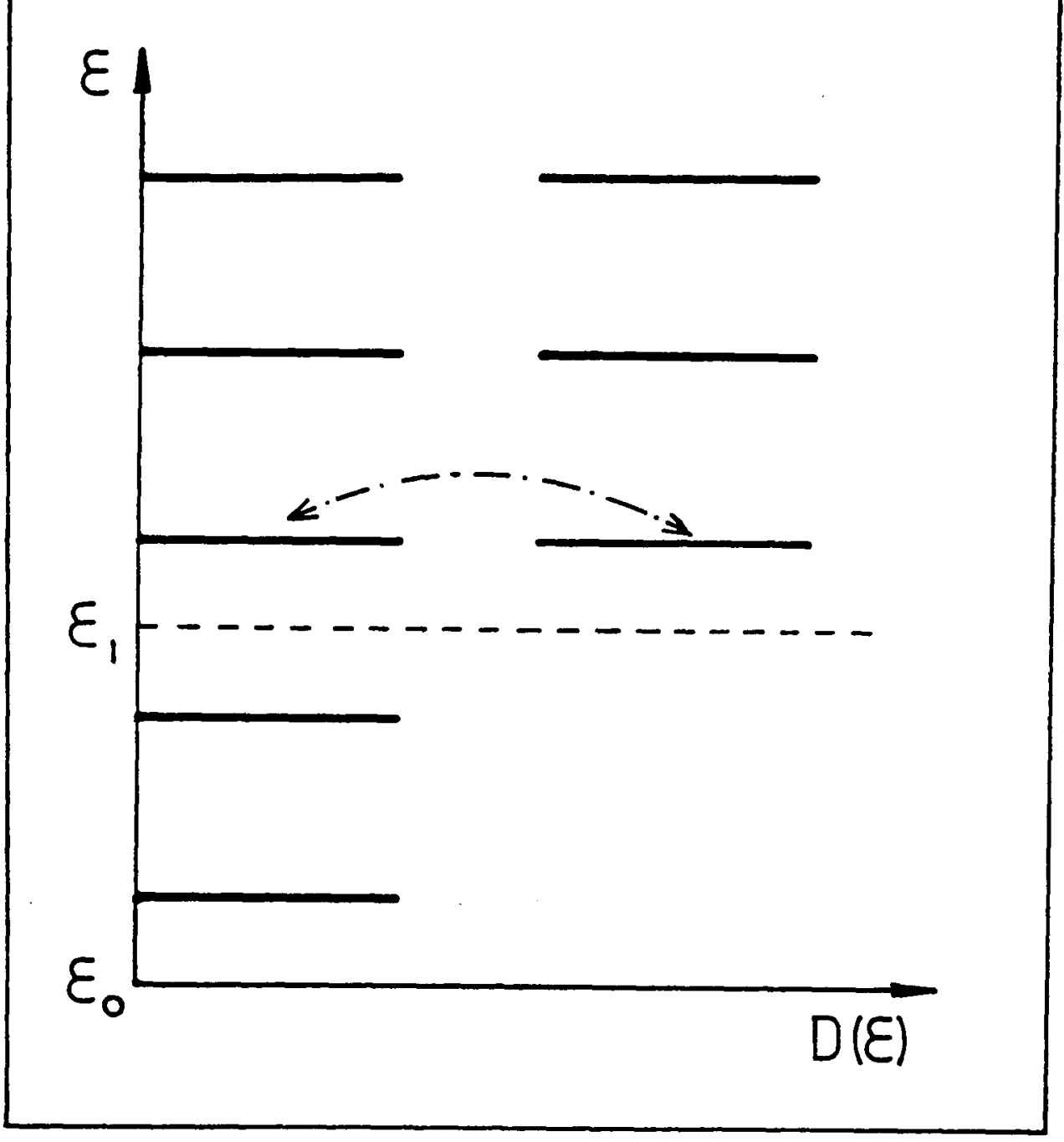

Figure 5.13 Schematic diagram of the inter-subband resonance condition.

condition Eqn. 5.4 does not hold the electron temperature will be somewhere between the lattice temperature and that required for optic phonon emission ( $100 \mathrm{~K}$ for $36 \mathrm{meV}$ phonons) and as the mobility increases rapidly with decreasing temperature in this region (in their calculations Walukiewicz et al. (1984) found a fourfold increase between $100 \mathrm{~K}$ and $50 \mathrm{~K}$ ) there will be minima in the resistivity whenever Eqn. 5.4 is satisfied.

Consider the diffusion picture where the electrons move up in energy until they encounter a strong scattering process. It can be seen that inter-subband scattering could limit the flow up in energy effectively at low electric fields and so prevent the emission of optic phonons. At higher electric fields the electrons would have more energy, be able to get past this barrier and so still emit phonons.

Measurements of $\mathrm{E}_{10}$ will be discussed in Sections 6.2\&3 and are shown in Fig. 6.1. This suggests values of $9.3 \mathrm{meV}$ and $14.5 \mathrm{meV}$, corresponding to fundamental fields of 5.7T and $8.8 \mathrm{~T}$, in G139 and G83 respectively (remembering that G83 is in the dark and G139 is partially photoexcited). These are both some 3T lower than we measure, but with a similar electron concentration dependence. The discrepancy is equivalent to 
an energy shift of $\sim 5 \mathrm{meV}$ and may be due to the higher temperatures at which our measurements are made. Although Stern \& Das Sarma (1984) found little change in $E_{10}$ with temperature, they neglected any change in the number of impurities that would be ionised, which may change $E_{10}$. To calculate the resonance fields the polaron-dressed mass has been used for each sample measured from cyclotron resonance at the relevent temperature. This is probably an underestimate and is certainly smaller than the magnetophonon mass, but the difference is unlikely to be larger than $\sim 5 \%$.

\subsection{SUMMARY}

Hot electron magnetophonon resonances have been directly observed in GaAs/GaAlAs heterojunctions between 30 and $100 \mathrm{~K}$ and their temperature and electric field dependences studied. The oscillation amplitude is seen to have a maximum at $60 \mathrm{~K}$, which is explained in a picture of diffusive transport. The low densities of states found between Landau levels at low temperatures is thought to limit the electron flow through energy space and hence reduce the resonance amplitudes. A similar arguement is used to explain why the higher harmonics change from maxima to HEMPR minima first. The resonance positions indicate phonon frequencies well below the expected LO value, as observed in normal magnetophonon resonance in heterojunctions.

The results have also been discussed in the light of calculations using the momentum balance equation and qualitative agreement has been found. In order to make a more quantitative comparison more experiments at high magnetic fields are required and specific sample parameters need to be used in the calculation.

Additional series of oscillations have been found when electrons have insufficient energy to emit optic phonons. These are thought to provide evidence for an enhancement of energy loss by resonant inter-subband scattering. 


\section{CHAPTER 6}

\section{INTER-SUBBAND SCATTERING IN GaAs-GaAlAs HETEROJUNCTIONS}

6.1 Introduction

6.2 Occupation of Higher Electric Subbands

6.2.1 Onset of Occupation

6.2.2 Measurement of $\mathrm{E}_{01}$

6.2.3 Effect on the Mobility

6.3 Magnetoresistance Measurements in Perpendicular Fields

6.3.1 Shubnikov-deHaas Oscillations from Multiple Subbands 172

6.3.2 The High Field Oscillations

6.3.3 The Low Field Oscillations

6.4 Effective g-Factors

$\begin{array}{lll}\text { 6.4.1 Enhancement of the g-factor } & 178\end{array}$

6.4.2 $\mathrm{g*}$ Measured from Shubnikov-deHaas Oscillations 180

6.5 Magnetoresistance Measurements in Parallel Magnetic Fields

$\begin{array}{lll}\text { 6.5.1 Depopulation of Upper Subbands } & 184\end{array}$

6.5.2 Measurement of Inter-Subband Scattering Rates 188

6.6 Effects of Inter-Subband Scattering on Shubnikov-deHaas Oscillations 6.6.1 Modulation of the Oscillations at Increased Temperatures 191

$\begin{array}{ll}\text { 6.6.2 Explanation of the Modulation } & 194\end{array}$

6.6.3 Acoustic Phonon Mediated Inter-Subband Scattering 197

$\begin{array}{llr}6.7 & \text { Summary } & 199\end{array}$ 


\section{$\underline{6.1 \quad \text { INTRODUCTION }}$}

In the last three chapters polar electron-LO phonon scattering has been discussed at great length. However, this is not the only scattering mechanism to affect the magnetoresistance of the 2DEG, especially at low temperatures. In the next three chapters some of the other mechanisms will be mentioned, principally low energy acoustic phonon interactions and inter-subband scattering. In the previous chapter an example was given of how inter-subband scattering may provide a resonant energy loss mechanism in the warm electron regime. This chapter studies inter-subband scattering in more detail showing that at low temperatures it is influenced by acoustic phonons and providing some methods of measuring the associated scattering rate. Chapter 7 is concerned with the scattering times which result from different mechanisms. Finally, Chapter 8 will present measurements of electron energy loss rates that demonstate emission of acoustic phonons at the cyclotron energy.

In high quality $2 \mathrm{D}$ systems the electron mobility will be limited by acoustic phonon scattering at temperatures below $\sim 60 \mathrm{~K}$ (when optic phonon scattering will become the dominant effect) so information concerning this interaction is of real interest. This is especially so in devices operating in the warm electron regime, where the carriers are not in thermal equilibrium with the lattice and the energy relaxation mechanisms play a crucial role in determining the device performance. At low temperatures a large part of this energy loss will be by acoustic phonon emission.

Section 1.3 discussed quantization in heterostructures and showed that in the absence of magnetic fields the confinement of the electron wavefunctions perpendicular to an interface results in the electron energy being restricted to several electric subbands. At very low temperatures the number of populated subbands will depend on the relative sizes of the Fermi energy, $E_{F}$, and the subband energies, $E_{i}$, and since $E_{F} \propto n_{s}$ multiple subbands will only be occupied in high carrier density samples. However, this does not mean that inter-subband scattering is only important in high density samples: at elevated temperatures higher subbands will be thermally populated even in the most sparsely populated heterojunctions. In narrow quantum wells the energy separation of 
the two lowest subbands, $E_{01}$, is large, meaning that upper subbands are rarely occupied, so this chapter will concentrate on measurements made in GaAs-GaAlAs heterojunctions.

Two types of experiment are considered :-

(i) Firstly, observations of the Shubnikov-deHaas oscillations in heterojunctions between $0.5 \mathrm{~K}$ and $4.2 \mathrm{~K}$. These have been performed as a function of both temperature and electric field in high density samples and provide evidence that acoustic phonon scattering may strongly influence the inter-subband scattering (Sections 6.6). It is also possible to extract values of $E_{01}$ (Section 6.3) and the spin-splitting of each subband (Section 6.4).

(ii) With the magnetic field in the plane of the 2DEG upper subbands can be depopulated, which allows comparison of intra- and inter-subband scattering to be made in Section 6.5.

\subsection{OCCUPATION OF HIGHER ELECTRIC SUBBANDS}

\subsubsection{Onset of Occupation}

As mentioned above more than one subband will only be occcupied at low temperatures when the carrier concentration is greater than a critical value $\left(\mathrm{n}_{\text {crit }}\right)$ which depends on $E_{01}$ and hence on the shape of the potential. A rough calculation of the Fermi energy in GaAs assuming a parabolic band and ignoring corrections due to many body effects gives :

$$
\begin{aligned}
\mathrm{E}_{\mathrm{F}} & =\frac{\pi \hbar^{2} \mathrm{n}_{\mathrm{s}}}{\mathrm{m}^{*}} \\
& \approx 3.5 \mathrm{meV} \text { per } 10^{11} \text { electrons } / \mathrm{cm}^{-2}
\end{aligned}
$$

where $\mathrm{m}^{*}$ has been taken as $0.068 \mathrm{~m}_{\mathrm{e}}$. Non-parabolicity of the conduction band will reduce this value of $E_{F}$ by $\sim 1 \%$. In narrow band gap materials, such as the II-VIs studied by Singleton et al. (1986), $\mathrm{m}^{*}$ is much smaller, while $E_{\mathrm{i}}$ scales as $\mathrm{m}^{*-1 / 3}$ (cf Eqn.1.9), giving rise to 4 or 5 populated subbands at carrier concentrations $\sim 10^{12} \mathrm{~cm}^{-2}$. However, in the wider gap materials of interest to this thesis it is rare to have more than two higher subbands occupied. 
Accurate self-consistent calculations of the subband energies in a GaAs-GaAlAs heterojunction were first made by Ando (1982a), who found a very strong dependence on $\mathrm{n}_{s}$ and $\mathrm{n}_{\text {dep }}$, the depletion charge density and predicted occupation of the upper subband for $\mathrm{n}_{\mathrm{s}}>7 \times 10^{11} \mathrm{~cm}^{-2}$ or $3 \times 10^{11} \mathrm{~cm}^{-2}$, with $\mathrm{n}_{\text {dep }}=5 \times 10^{10} \mathrm{~cm}^{-2}$ and $1 \times 10^{9} \mathrm{~cm}^{-2}$ respectively. Stern \& Das Sarma (1984) studied the effects of changes in these, and other, parameters in more detail and their results are reproduced in Fig 6.1. In the Philips grown samples the depletion charge is $\sim 3.5 \times 10^{10} \mathrm{~cm}^{-2}$, reducing to $\sim 1.5 \times 10^{10} \mathrm{~cm}^{-2}$ on illumination with a red LED (Harris et al. 1987).

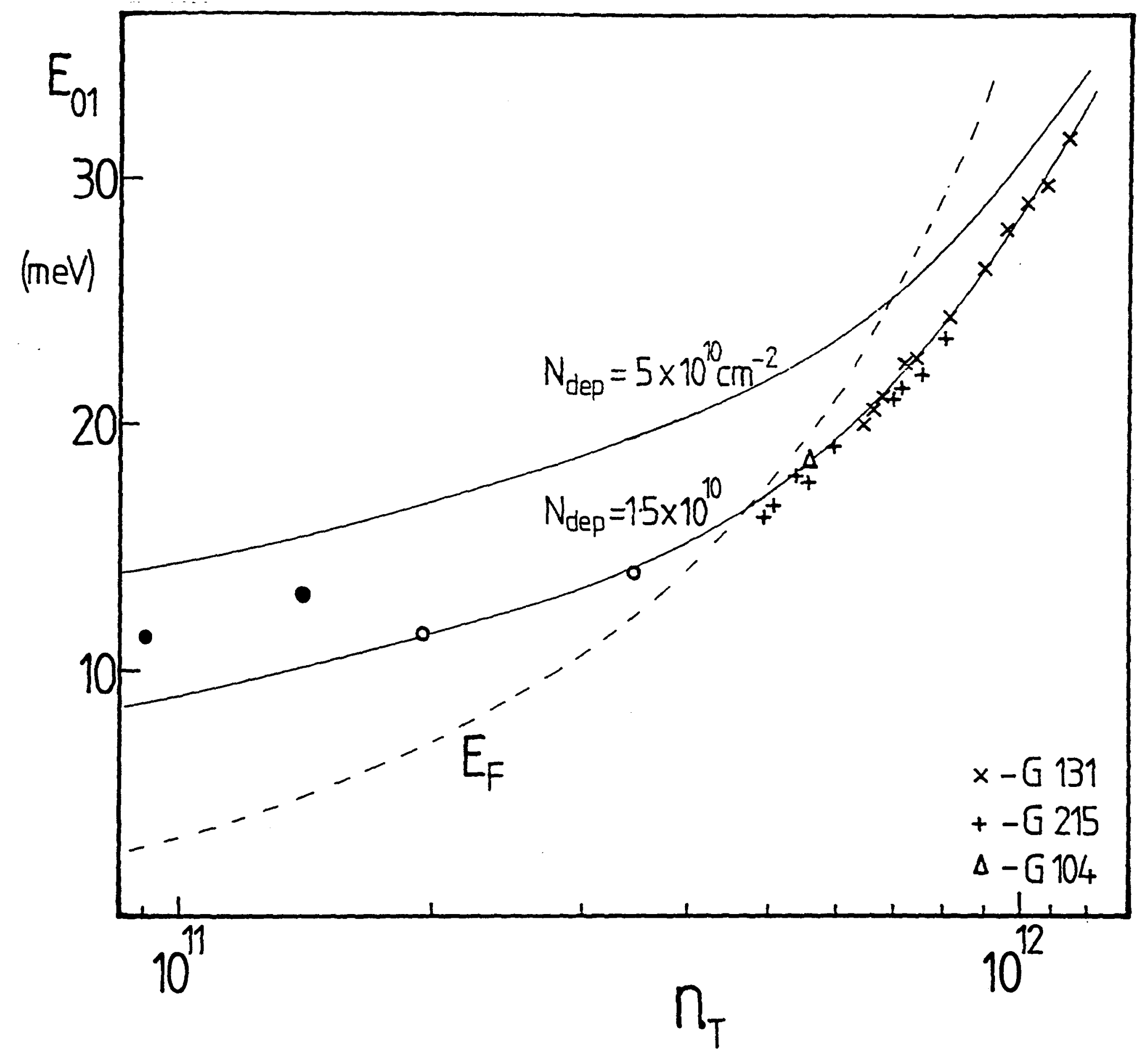

Figure 6.1 Separation of the first two electric subbands $\left(E_{01}\right)$ as a function of total carrier concentration. Solid lines are calculations of Stern \& Das Sarma. Dashed line represents the Fermi energy (Eqn.6.1). Circles are from cyclotron resonance measurements of Hopkins - open circles after illumination. 
Fig. 6.1 then suggests that as $n_{s}$ increases from $10^{11}$ to $10^{12} \mathrm{~cm}^{-2} E_{01}$ should increase from $\sim 10 \mathrm{meV}$ to $\sim 28 \mathrm{meV}$. On increasing the temperature Stern \& Das Sarma found the subband separation hardly changed, except at the highest carrier densities. However, they also calculated the fractional occupancy of each subband showing $\sim 10 \%$ of the electrons would be in the $E_{1}$ level at $77 \mathrm{~K}$ irrespective of $n_{s}$.

Walukiewicz et al. (1984) performed a variational calculation using a triangular potential well and found reasonable agreement with Ando (1982a). Their expression for the subband separation reduces to:-

$$
E_{01}=1.19 \times 10^{-7}\left(n_{s}+\frac{32}{11} n_{d e p}\right)^{0.69} \mathrm{meV}
$$

or approx.

$$
\mathrm{E}_{01} \propto \mathrm{n}_{\mathrm{s}}{ }^{2 / 3}
$$

For any carrier concentration greater than $\mathrm{n}_{\text {crit }}$ both subbands will be occupied. Then from Eqn. 6.1 and requiring equality of the Fermi level in each subband:-

$$
\frac{\pi \hbar^{2}}{\mathrm{~m}^{*}} \mathrm{n}_{0}=\frac{\pi \hbar^{2}}{\mathrm{~m}^{*}} \mathrm{n}_{1}+\mathrm{E}_{01}
$$

and by using the approx. form Eqn. 6.2a, writing $n_{T}=n_{0}+n_{1}$, and remembering $\mathrm{n}_{1}=0$ when $\mathrm{n}_{0}=\mathrm{n}_{\text {crit }}$ gives:

$$
\begin{aligned}
n_{1} & =\frac{1}{2} n_{T}\left(1-\left[\frac{n_{\text {crit }}}{n_{T}}\right]^{\frac{1}{3}}\right) \\
& \approx \frac{1}{6}\left[n_{T}-n_{\text {crit }}\right] \quad \text { for small } n_{1}
\end{aligned}
$$

\subsubsection{Measurements of $E_{01}$}

Experimentally $E_{01}$ has been measured by a variety of optical techniques. Abstreiter \& Ploog (1979) performed resonant Raman scattering on back-gated accumulation layers and found a peak at $13.7 \mathrm{meV}$ for a sample with an aluminium fraction of 0.36 . Increasing the gate voltage caused the peak to disappear as the upper subband depopulated. There have been several studies of luminescence (e.g. Pinczuk et al. 1979, Meseguer et al. 1987), but these have all been performed on quantum well structures 
where the subband energies are fixed by the well widths.

Subband-Landau level coupling can be seen in cyclotron resonance measurements as a discontinuity in the effective mass, together with an increase in linewidth, when the sample normal is tilted a few degrees from the magnetic field (Schlesinger et al. 1983). Since the tilt angles are only small the diamagnetic shift, (considered below) will be $\leqslant 0.1 \mathrm{meV}$, however there will still be a shift due to depolarisation and exitonic effects giving an energy $2 \sim 3 \mathrm{meV}$ greater than $E_{01}$ (Ando 1982a). Hopkins (1987) measured the subband separation in samples $G 139, \mathrm{G} 63$ and $\mathrm{G} 29$ both in the dark and after photoexcitation. The data is included on Fig. 6.1 showing a larger subband splitting in the dark, in good agreement with the calculations of Stern \& Das Sarma (1984) using the relevent depletion charge densities, mentioned above. Similar $n_{s}$ dependences in GaAs-GaAlAs hetrerojunctions were also measured by Rikken et al. (1986) who found an increase of $E_{01}$ with $n_{s}$ when the carrier concentration was varied by persistent photoconductivity, but the opposite behaviour when $n_{s}$ was changed with a back-gate voltage.

The dashed line of Fig. 6.1 represents the Fermi energy calculated for all electrons in one subband (Eqn. 6.1), so, guided by data of Hopkins to establish the depletior charge, population of the $E_{1}$ subband would be expected for $n_{s} \gtrsim 5 \times 10^{11} \mathrm{~cm}^{-2}$. In Section 6.3.2 measurements of $E_{01}$ from Shubnikov-deHaas oscillations will be presented and it is these that appear in Fig. 6.1.

\subsubsection{Effects on the Mobility}

Once the Fermi energy enters the upper subband an additional inter-subband scattering channel will become available and the mobility measured in zero-field will be reduced. Walukiewicz et al. (1984) found the inter- and intra-subband scattering rates were very similar in their calculations of phonon contributions to the d.c. mobility. Mori \& Ando (1980) calculated that $\mu$ should drop by a factor of 3 once $E_{1}$ was populated. This drop was first observed in a GaAs-GaAlAs heterojunction by Störmer et al. in 1981, but only by $30 \%$. Measurements for G131, shown in Fig. 6.2 (see also Harris et al. 1987 and Fletcher et al. 1988), clearly demonstrate an abrupt 
change in mobility, $\sim 15 \%$ in this case, with $\mathrm{n}_{\text {crit }} \sim 6 \times 10^{11 \mathrm{~cm}^{-2}}$. The much smaller reduction is due to a smearing of the band edge, as calculated by Cantrell \& Butcher (1985). Harris et al. (1987) found that changing the wavelength of the LED used to excite carriers caused $\mathrm{n}_{\text {crit }}$ to vary from 5.0 to $6.4 \times 10^{11} \mathrm{~cm}^{-2}$ in Sample G131. Taking this result, together with that of Rikken et al. (1986) discussed above, shows how sensitive $E_{01}$ is to the exact charge distribution. Once the upper subband starts to be populated with electrons there will be additional screening of the impurity potential and so $\mu$ will increase again.

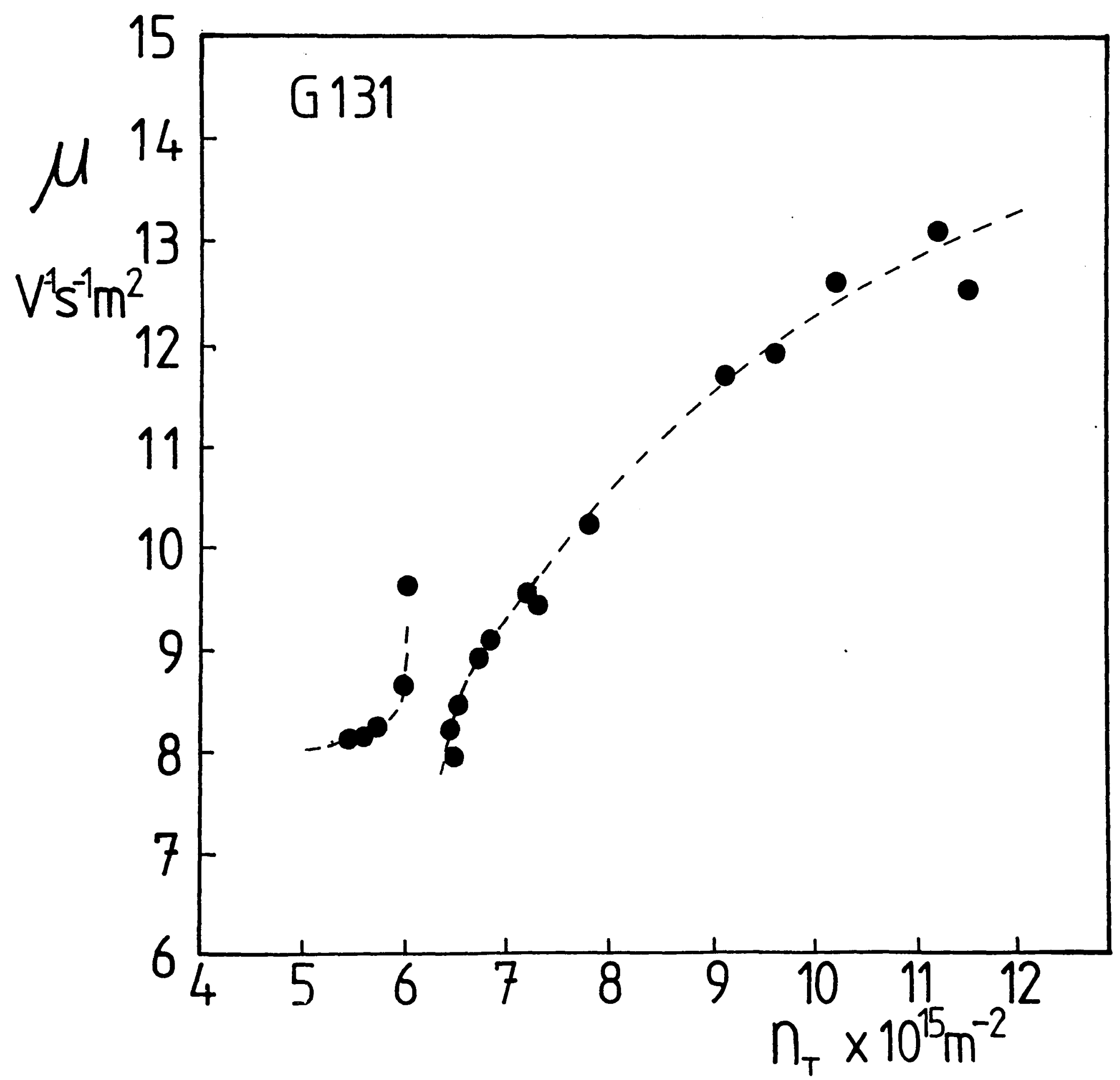

Figure 6.2 Carrier concentration dependence of the mobility in $\mathrm{G} 131$ at $4.2 \mathrm{~K}$, showing an abrupt change as the upper subband starts to be populated. 
Figure 6.3 Low field Shubnikov-deHaas oscillations from G215 showing two subbands.

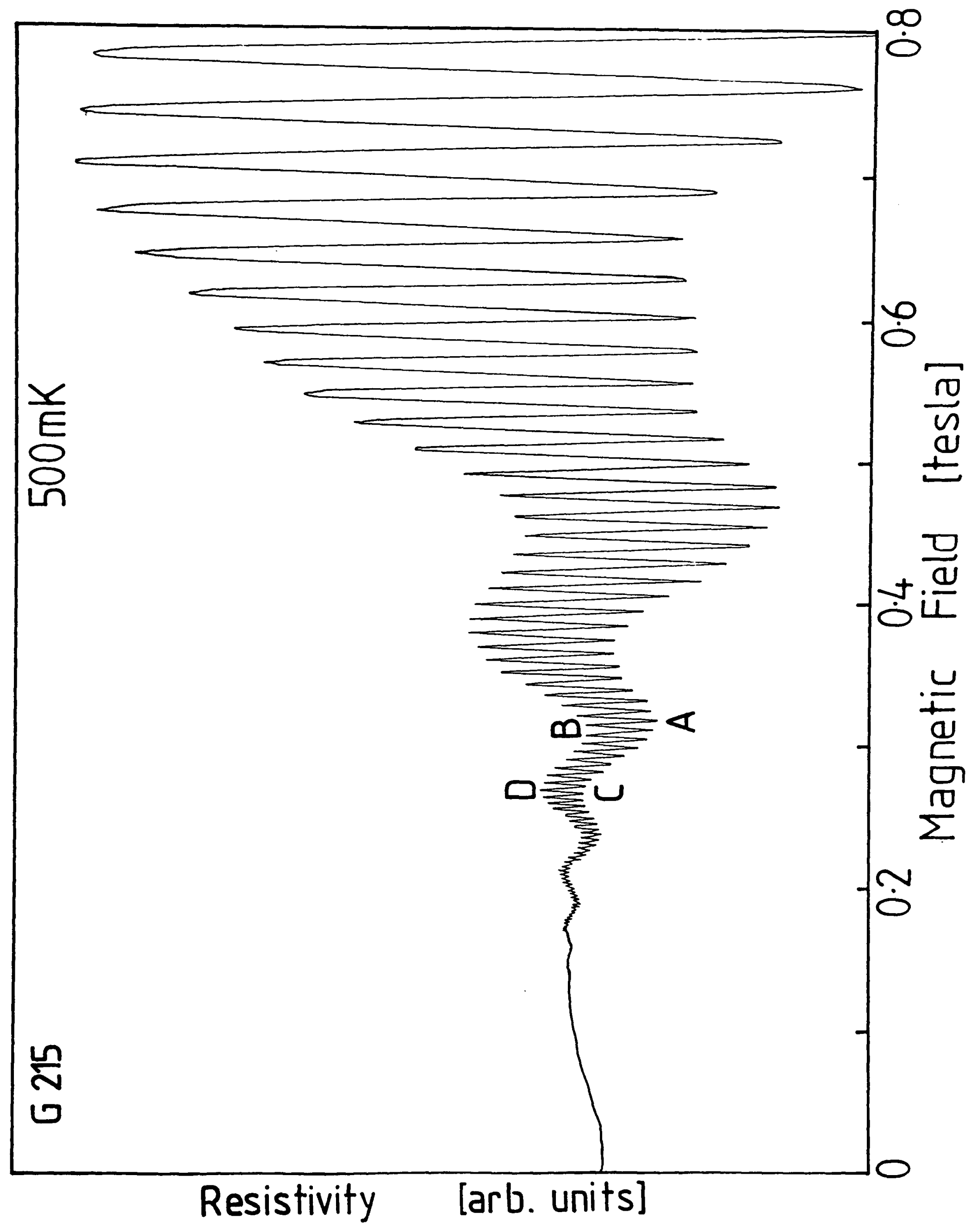




\subsection{MAGNETORESISTANCE MEASUREMENTS IN PERPENDICULAR FIELDS}

Population of the upper subband can also be detected from low temperature magnetoresistance measurements, in magnetic fields either perpendicular (this section) or parallel (Section 6.5) to the plane of the 2DEG.

\subsubsection{Shubnikov-deHaas Oscillations from Multiple Subbands}

In perpendicular magnetic fields the magnetoresistance shows Shubnikov-de Haas oscillations, as discussed in Section 2.2. At low fields $(<2 \mathrm{~T})$ there is a single series of oscillations periodic in $1 / B$, but on population of an upper subband an additional periodicity appears. A typical example is shown for a GaAs-GaAlAs heterojunction in Figure 6.3 - sample $\mathrm{G} 215$ at $500 \mathrm{mK}$ after full illumination. This shows what one would conventionally expect, namely that there are two independent series of oscillations present which contribute additively to the conductivity (and, since $\sigma_{x y} \gg \sigma_{x x}$ in the region of interest, also the resistivity). These will remain independent as long as the Landau levels are not sufficiently well separated to cause the Fermi energy to move. The two periodicities allow the carrier concentrations in each subband to be deduced, in this case $7 \%$ are in the upper level. Note that oscillations from the $\mathrm{E}_{0}$ subband with a Landau index of up to $\sim 90$ can be discerned.

\subsubsection{The High Field Oscillations}

At higher fields the oscillations become more complex, as shown in Figure 6.4 , as a result of the irregular movement of the Fermi level between the Landau levels of the two occupied subbands. An example of this is the appearance of spin-splitting, accompanied by a further weak structure on the high field side of each peak due to $E_{F}$ entering the lowest spin-split level of the upper subband. This peak asymmetry is apparent even before two series of oscillations can be seen, i.e. with very small upper subband population. Comparison of Fig. 6.4 with the fan diagram of Landau level energy in the magnetic field, Figure 6.5, shows how $E_{F}$ is unable to escape from the lowest upper subband levels. The spin-splitting of the upper subband levels can be seen in Figure 6.3 from the minimum in the high frequency oscillations at $0.6 \mathrm{~T}$, 


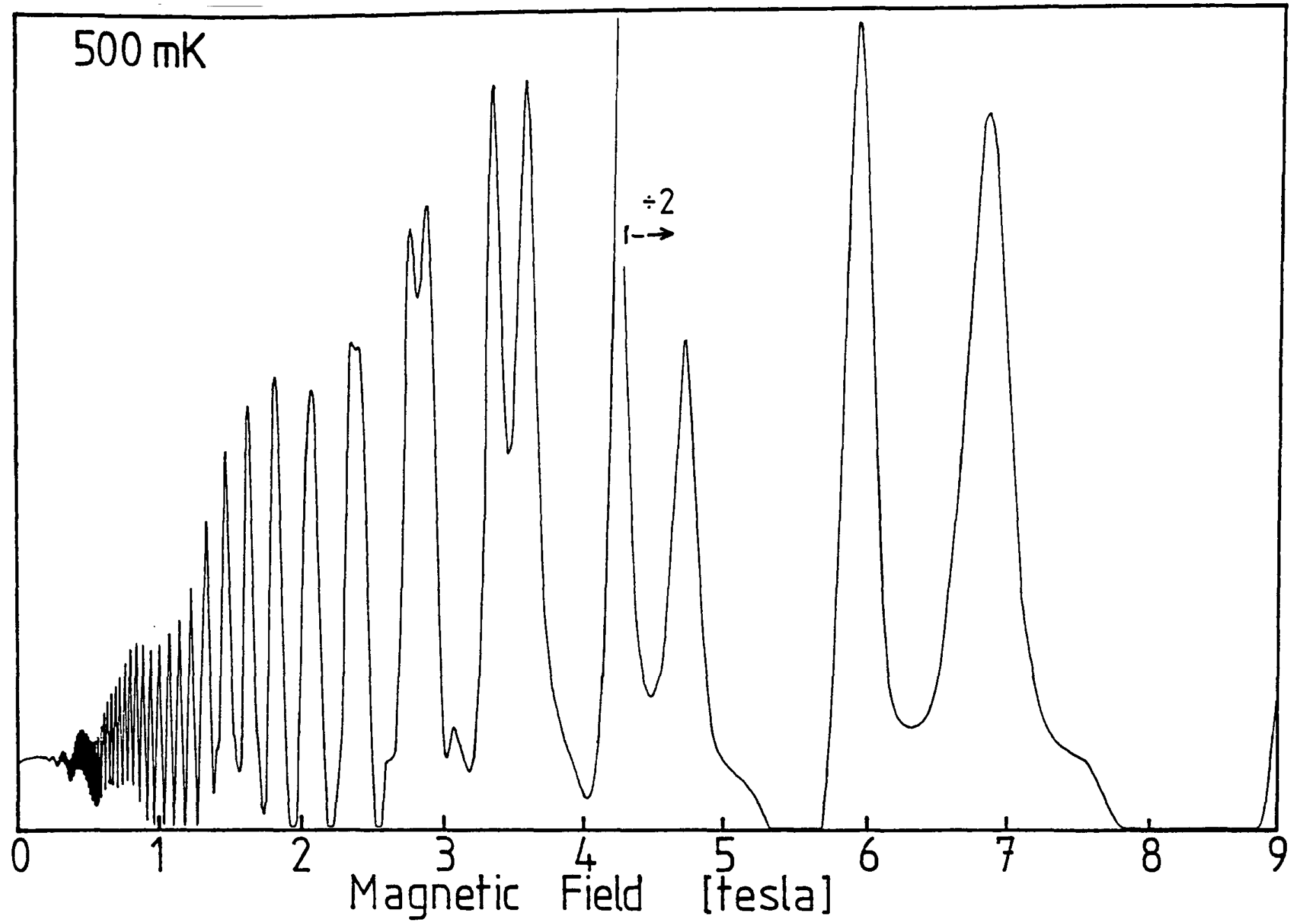

Figure 6.4 Shubnikov-deHaas oscillations at higher magnetic fields than Fig.6.3 showing spin splitting and peak asymmetry. Magnification is halved above $4 \mathrm{~T}$.

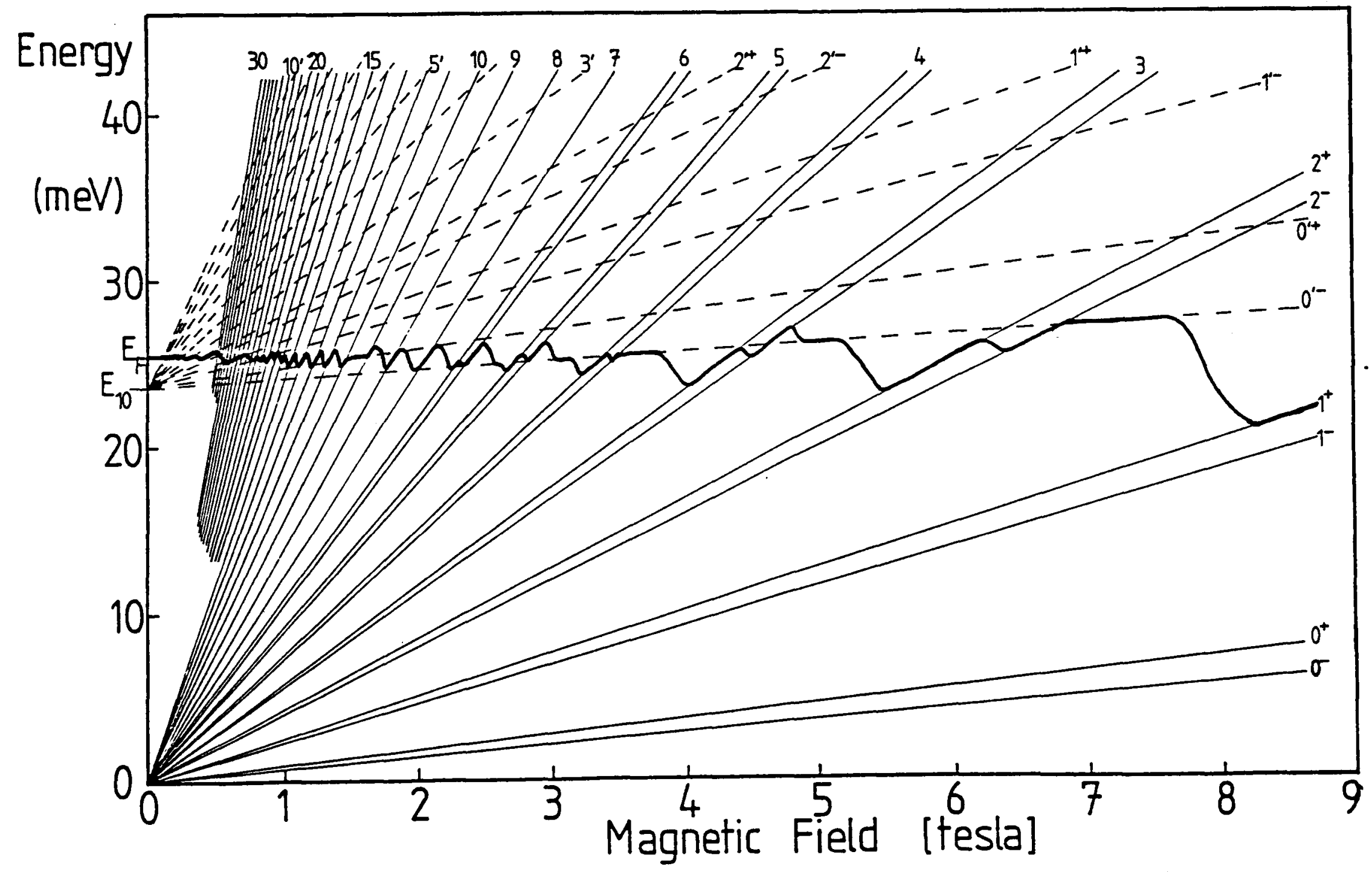

Figure 6.5 Movement of the Fermi energy through thye Landau levels of the lower (solid lines) and upper (dashed) subbands. Numbers represent the Landau index with spin splitting shown as \pm . 
although it is not seen in the lower levels until 2.4T. Smith et al. (1988) also saw spin-splitting in the upper subband at $1.1 \mathrm{~T}$ under similar conditions. They noted that this implies a significantly enhanced $\mathrm{g}$-factor and a long single particle relaxation time (of which more in Chapter 7) in the upper subband. In Section 6.4 the Shubnikov-deHaas oscillations will be used to deduce values for $\mathrm{g}^{*}$ in each subband and it is these values that have been used to draw Fig. 6.5.

\section{Measurements of $E_{01}$}

Comparison of the oscillations with fan diagrams for each subband can also be used to find values of $E_{01}$. At low magnetic fields $E_{F}$ will be approximately constant so it can be determined from the positions of high Landau index oscillations from the ground subband. Then the oscillations from the upper subband allow $E_{1}$ to be fixed relative to $E_{F}$, and hence $E_{10}$ is determined. The results are included in Fig. 6.1 and show extremely good agreement with theory.

\subsubsection{The Low Field Oscillations}

The periodicities of the low field oscillations allow the populations of the $E_{0}$ and $E_{1}$ subbands, $n_{0}$ and $n_{1}$, to be deduced from Eqn. 2.9. At higher fields, where truly flat bottomed minima are observed, all the carriers are in the lowest subband and the periodicity gives the total carrier concentration, $\mathrm{n}_{\mathrm{T}}$. Figure 6.6 shows how the upper subband population varies with $\mathrm{n}_{\mathrm{T}}$ in two GaAs-GaAlAs heterojunctions - G131 \&. G215. The data points show that it is possible to observe $\mathrm{SdH}$ oscillations from the upper subband provided $n_{1}>2 \times 10^{10} \mathrm{~cm}^{-2}$, although evidence for occupation was visible at lower $n_{T}$ from the peak asymmetry at higher fields. In both cases $n_{1} \propto\left(n_{T}-n_{\text {crit }}\right)$, with the constant of proportionality being 0.13 for $\mathrm{G} 215$ and 0.16 for G131 in good agreement with Eqn. 6.4a. The onset of upper subband population is found from extrapolation as $n_{\text {crit }}=4.0 \times 10^{11} \mathrm{~cm}^{-2}$ in $\mathrm{G} 215$ and $5.4 \times 10^{11 \mathrm{~cm}^{-2}}$ in $\mathrm{G} 131$. The reason for earlier population in G215 is that the potential confining the electrons to the interface is shallower as a result of the $\mathrm{n}^{-}$buffer layer. 


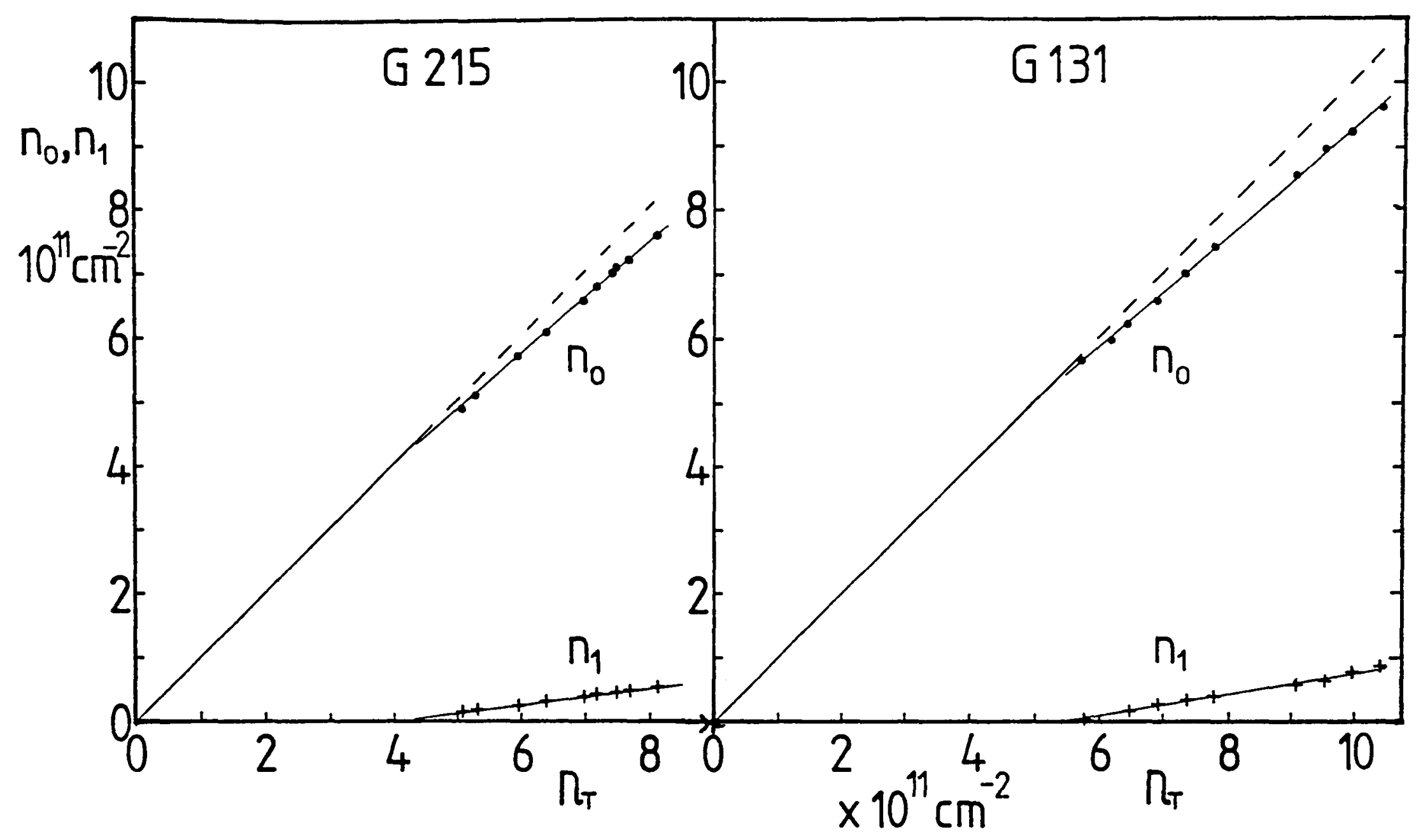

Figure 6.6 Population of the first two electric subbands as a function of total carrier concentration in two GaAs-GaAlAs heterojunctions.
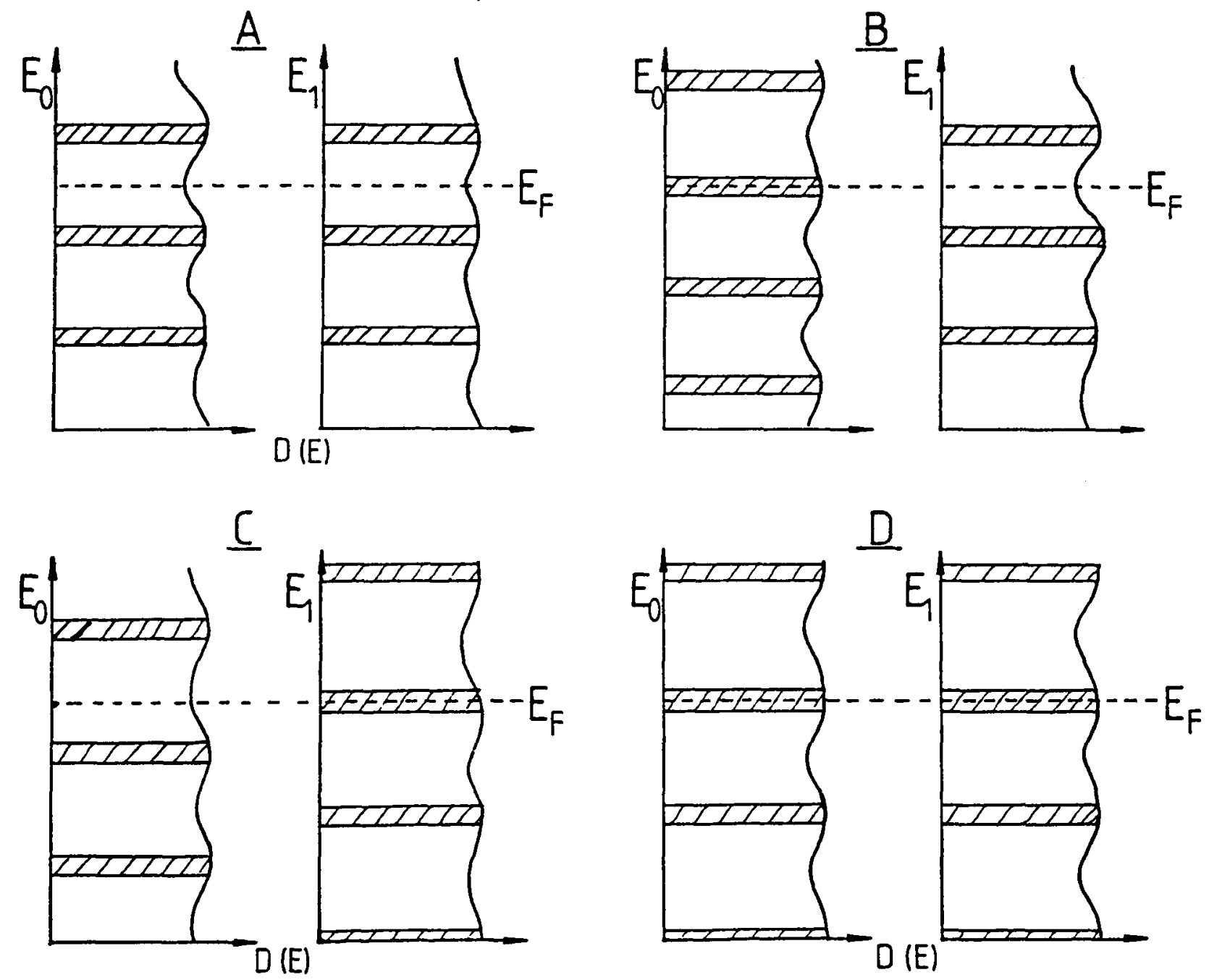

Figure 6.7 Schematic view of the Fermi level in the density of states of each subband corresponding to the positions $A, B, C \& D$ on Fig.6.3. Hatched regions represent the extended states, responsible for conduction. 


\section{Additive Series}

In these samples $\rho_{x y}>\rho_{x x}$ for $B>0.1 T$, so at all the magnetic fields where oscillations are seen the high field limit discussed in Section 2.2 applies ie. the resistivity is directly proportional to the scattering rate which depends on the density of states at $E_{F}$ and so oscillates in magnetic field. As there is a factor of $\sim 20$ between the carrier concentrations of the two subbands the Landau levels of $E_{o}$ move rapidly through $E_{F}$, giving the high frequency oscillations, while the $E_{1}$ Landau levels move only very slowly. This is illustrated by a comparison of the resistivity at points A,B,C\&D in Figure 6.3 with the schematic view of the densities of states at $E_{F}$ in each case presented in Figure 6.7: at point $A$ there is a minimum DoS at $E_{F}$ giving a minimum scattering rate and hence a minimum resistivity; $B$ and $C$ have $E_{F}$ lying in one subband only and at both positions the magnitude of $\rho_{x x}$ is similar - suggesting that it is only the total number of available conducting states that is important and in turn that the mobilities of each subband must be similar; at $\mathrm{D}$ there is a maximum DoS and so maximum $\rho_{\mathbf{x x}}$.

This addition of the oscillations may be shown from considerations of the contributions to the total conductivity from each subband. That we actually see oscillations from the upper subband means $\Delta \rho / \rho$ is not simply $\left(\Delta \sigma_{0}+\Delta \sigma_{1}\right) /\left(\sigma_{0}+\sigma_{1}\right)$ [since, with $\sim 20$ times more carriers in $E_{0}, \Delta \sigma_{1} \ll \Delta \sigma_{0}$ ], so we will perform a calculation. In the relaxation time approximation with a magnetic field, $\mathrm{B}$, perpendicular to the conducting layer the transport current is:

$$
j_{x}=e\left[\frac{n_{0} \mu_{0}}{1+\mu_{0}^{2} B^{2}}+\frac{n_{1} \mu_{1}}{1+\mu_{1}^{2} B^{2}}\right] E_{x}+e B\left[\frac{n_{0} \mu_{0}^{2}}{1+\mu_{0}^{2} B^{2}}+\frac{n_{1} \mu_{1}^{2}}{1+\mu_{1}^{2} B^{2}}\right] E_{y}
$$

where $n_{i}, \mu_{i}$ are the carrier density and mobility in each subband. From the relationship $j_{i}=\sigma_{i j} E_{j}$ the diagonal and off-diagonal components of the conductivity tensor, $\sigma_{\mathrm{xx}}$ and $\sigma_{\mathrm{xy}}$ can be identified and then $\rho_{\mathrm{ij}}$ calculated from inversion of the tensor relation Eqn. 2.1. In the high field limit $(\mu B \geqslant 1)$ Eqns. 6.5 \& 2.1 reduce after a little algebra to:

$$
\rho_{x x}=\frac{n_{0} \mu_{1}+n_{1} \mu_{0}}{\mathrm{e} \mu_{0} \mu_{1}\left(n_{0}+n_{1}\right)^{2}}
$$


Then writing the conductivity of the $\mathrm{i}^{\text {th }}$ subband as $\sigma_{\mathrm{i}}=\mathrm{en}_{\mathrm{i}} \mu_{\mathrm{i}}$ the oscillations in the resistivity, in the same limit, will be given by:

$$
\frac{\Delta \rho}{\rho}=\frac{-1}{n_{0} \mu_{1}+n_{1} \mu_{0}}\left[n_{0} \mu_{1} \frac{\Delta \sigma_{0}}{\sigma_{0}}+n_{1} \mu_{0} \frac{\Delta \sigma_{1}}{\sigma_{1}}\right]
$$

where it is assumed that the number of carriers in each subband remains constant, so $\Delta \sigma_{\mathrm{i}}=e \mathrm{n}_{\mathrm{i}} \Delta \mu_{\mathrm{i}}$. In a modulation doped heterostructure the carrier density in the 2DEG is fixed by the number of ionised donors in the GaAlAs, which should be determined by the surface potential, so this aproximation is reasonable. In a magnetic field the Fermi energy will move through the Landau levels of the 2DEG as shown in Fig. 6.5. Alternatively, one could assume a fixed Fermi energy - a better assumption for surface accumulation layers - in which case $E_{F}$ is pinned by the surface states and the carrier density must adjust itself accordingly.

\section{Deductions Regarding Level Broadening}

In the present situation $n_{0} / n_{1} \sim 20$ whereas the mobilities in each subband never differ by more than a factor of 4 , so the fact that oscillations from both subbands contribute with a similar weight suggests the fractional change in conductivity is much larger in the upper subband. This would mean less broadening of the $E_{1}$ Landau levels - a point which will be returned to in the next chapter.

It is interesting to consider the lowest magnetic field at which one might expect to observe Shubnikov-de Haas oscillations. For GaAs Eqn. 1.21 gives the separation of Landau levels as $\sim 20 K / T$, so in order to observe oscillations at a field B (in tesla) the half-width of the broadened levels, $\Gamma$ in kelvin, must be less than 20B. In high mobility material the broadening will be limitted by disorder at low temperature and is related to the single-particle relaxation time, $\tau_{s}$, by:-

$$
\Gamma=\frac{\hbar}{2 \tau_{s}}
$$

$T_{s}$ has been calculated by Das Sarma \& Stern (1985) and measured by several groups and will be discussed in detail in Chapter 7. Figure 6.3 shows well resolved oscillations from the lower subband down to $\sim 0.2 \mathrm{~T}$, ie. $\Gamma \sim 4 \mathrm{~K}$ and oscillations from the upper subband can be seen to smaller fields. This again suggests narrower levels in the upper 
subband. For G215 $\tau_{\mathrm{s}}$ is found in Chapter 7 to be $0.8 \mathrm{ps}$ and $2.1 \mathrm{ps}$ in the $\mathrm{E}_{0}$ and $\mathrm{E}_{1}$ subbands, giving $\Gamma_{0}=4.7 \mathrm{~K}$ and $\Gamma_{1}=1.8 \mathrm{~K}$ in reasonable agreement with the data.

Attempts at directly measuring the broadening of the density of states, eg. from deHaas-van Alphen effect by Eisenstein et al. (1985) and capacitance measurements Smith et al. (1985), all find a much larger $\Gamma \sim 0.9 \mathrm{meV}$ (ie. $\sim 9 \mathrm{~K}$ ). [See Section 1.5]. This could be explained by the superior quality of our samples (at least a factor of 2 in d.c. mobility), but it has been suggested that in transport measurements only the width of extended states will be revealed whereas in measurements of a thermodynamic quantity, such as magnetisation, all states will contribute equally. It would thus be interesting to determine $\Gamma$ by several different methods on the same sample.

\subsection{EFFECTIVE $\mathrm{g}$-FACTORS}

Figure 6.4 showed strong spin splitting of the Landau levels and it was found that the size of this splitting was a crucial quantity in drawing a realistic fan chart (Fig. 6.5). From Eqn. 1.24 the splitting is given by $\mathrm{g}^{*} \mu_{\mathrm{B}} \mathrm{B}$ and in the absence of electron-electron interactions $\mathrm{g}^{*}=\mathrm{g}$, the bare Landé $\mathrm{g}$-factor, however when the many-body interactions are acounted for a much enhanced value of $g^{*}$ may result.

\subsubsection{Enhancement of the g-Factor}

An exchange enhancement of the electronic $g$-factor in $2 D$ systems was first proposed by Janak (1969) to explain measurements on silicon MOSFETS where Fang \& Stiles (1968) found a considerably larger $g$ than the bulk value of 2 . Since then there have been many studies by a variety of workers [see Usher (1988) for a review], both using transport and optical measurements and theoretical studies of this interesting consequence of many-body interactions. However, the resulting values of the $g$-factor differ widely, with strongly enhanced values only being found in transport measurements.

Janak (1969) explained the observation of the bare $g$-factor in electron-spin-resonance experiments by considering the two stages of an electronic spin-flip process. Initially there is the actual change of spin, which is a rapid process. From the Pauli Exclusion Principle the spatial wavefunction then also has to change, which will 
influence the other electrons in the system which then have to rearrange themselves. This return to an equilibrium state is much slower. In ESR the photon absorption and cosequent spin flip is so rapid that surrounding electrons do not have time to respond to their new spin-flipped neighbour and so the photon energy is just $g \mu_{B} B$, with $g$ being the bare $g$-factor. By contrast, in transport measurements the magnetic field is swept so slowly that the electrons are always in their equilibrium configuration. Hence these experiments measure the energy involved in both the fast and slow processes and give the enhanced value, $\mathrm{g}^{*}$. Using ESR a bare $\mathrm{g}$-factor 0.44 was found by Herrmann \& Weisbuch (1977) in bulk GaAs and by Stein et al. (1983) in heterojunctions.

Englert et al. (1982) showed, using the work of Ando \& Uemura (1974), that the enhancement in the $\mathbf{N}^{\text {th }}$ Landau level is:

$$
\mathrm{g}^{*} \mu_{\mathrm{B}} \mathrm{B}=\mathrm{g} \mu_{\mathrm{B}} \mathrm{B}+\mathrm{E}_{\mathrm{ex}} \sum_{1}\left(\mathrm{n}_{\mathrm{i} \uparrow}-\mathrm{n}_{\mathrm{i} \downarrow}\right)
$$

where $E_{e x}$ is the exchange energy and $n_{i \uparrow}-n_{i \downarrow}$ are the populations of electrons in the $i^{\text {th }}$ Landau level with $\uparrow$ and $\downarrow$ spins. Thus if there is no difference in spin populations only the bare $g$-factor will be measured and to see a considerable enhancement there must be a large population difference between levels with spin $\uparrow$ and spin $\downarrow$.

$g^{*}$ may be evaluated from transport measurements by a variety of ways include tilted field coincidence measurements and activation studies, however it will only be observed in Shubnikov-deHaas measurements if $\mathrm{g}^{*} \mu_{\mathrm{B}} \mathrm{B}$ is greater than the broadening of the Landau levels and estimates (Nicholas et al. 1982, Englert et al. 1982) show that this requires an enhanced $g$-factor of around 5 . The spin-splitting depends on the total field value, whereas the Landau level separation is only sensitive to the component perpendicular to the 2DEG, so there will be a certain angle at which these are equal giving an easily recognisable pattern to the resistivity. However, this usually occurs at $\sim 80^{\circ}$, so high magnetic fields are required unless $\Gamma$ is fairly small. From this method Nicholas et al. (1988) found an enhanced $g^{*} \sim 2$ decreasing as the filling factor, $"$, increased. They also studied activated conduction between the two spin-split levels of a particular Landau level and from the temperature dependence of the minimum resistivity deduced a value for the energy gap and hence $g^{*}$. For a sample with $8 \times 10^{10} \mathrm{~cm}^{-2}$ 
carriers they found $\mathrm{g}^{*}=6.2$. Usher (1988) also studied activation energies and using samples G139, G156 and G148 found $\mathrm{g}^{*}=7.3$. Data at $\nu=1$ and $\nu=3$ gave the same value. At even integral filling factors there will be equal numbers of electrons in each spin state and so only the bare $\mathrm{g}$-factor is measured.

\subsection{2 $\mathrm{g}^{*}$ Measured from Shubnikov-deHaas Oscillations in $\perp^{\mathrm{r}}$ Fields}

It is reasonable to assume that the depth of a spin-split minimum will depend on the separation of spin-polarised levels in just the same way as the depth of minima at integral filling factors depends on the Landau level spacing. Thus the magnetic fields at which the percentage modulation of the resistivity by spin-splitting $\left(B_{1}\right)$ and by Landau level splitting $\left(B_{2}\right)$ are equal can be used to find a value of $g^{*}$, from the required equality of energy separation:

$$
\mathrm{g}^{*} \mu_{\mathrm{B}} \mathrm{B}_{1}=\frac{\hbar \mathrm{e}}{\mathrm{m}^{*}} \mathrm{~B}_{2}
$$

$$
\text { ie. } \quad \mathrm{g}^{*} \quad=29.4 \frac{\mathrm{B}_{2}}{\overline{\mathrm{B}}_{1}} \text { for GaAs }
$$

This follows from the same principle as the coincidence method, where $6.10 \mathrm{a}$ would be satisfied by rotating the sample until $B_{2} / B_{1}=\cos \theta$. Instead a field $B_{2}$ is found where $\Delta \rho / \rho$ is egual to the depth of the spin split minimum divided by the peak height at $B_{1}$. As the low field oscillations (around $B_{2}$ ) increase exponentially this method is quitesensetive. Values of $\mathrm{g}^{*}$ can be calculated in both subbands in the temperature regime where the contributions to the resistivity are additive. I find that for G215 with $\mathrm{n}_{0}=7.2 \times 10^{11} \mathrm{~cm}^{-2}$ and $\mathrm{n}_{1}=4.5 \times 10^{10} \mathrm{~cm}^{-2}$ the $\mathrm{g}$-factor is enhanced over the bare value by a factor of $\sim 8$ in the lower subband and by a colossal 32 for electrons in the upper subband! (ie. $\mathrm{g}^{*}=3.5$ and 14 respectively.)

As the results of Usher (1988) lie between the present values for the upper and lower subbands of G215 it may be that $g^{*}$ depends on carrier concentration, so it was measured for the lower subband in a number of samples with $\mathrm{n}_{0}$ from 0.9 to $8.7 \times 10^{11} \mathrm{~cm}^{-2}$, and in the upper subband of $\mathrm{G} 131$ for $0.3<\mathrm{n}_{1}<0.8 \times 10^{11} \mathrm{~cm}^{-2}$.

The results are given in Fig. 6.8 with the filling factor used indicated beside each 
Figure 6.8 Effective g-factor measured from Shubnikov-deHaas oscillations as a function of carrier concentration in several GaAs-GaAlAs heterojunctions. The dots are from the upper subband and the crosses from the lower subband. Numbers beside the points indicate the filling factor.

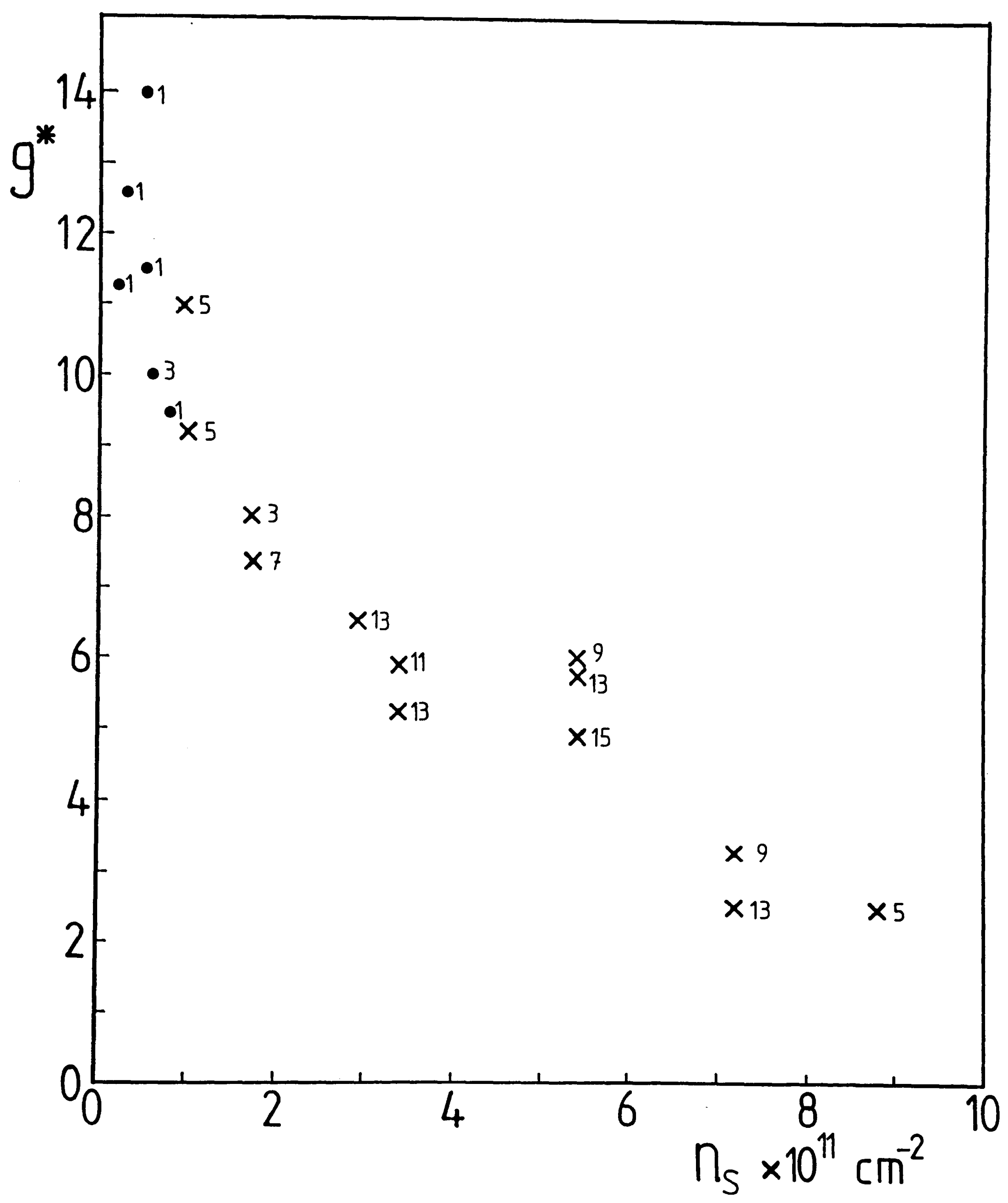


point. Although the absolute values deduced may be subject to fairly large errors, due to estimating the relative strengths of the minima, the general trend is striking. It shows a fairly smooth variation with carrier concentration and more enhanced $g^{*}$ at lower filling factors. The first point suggests that the very large $g^{*}$ found in the upper subband is merely a consequence of the low carrier density and not due to a fundemental difference in the exchange interaction between the subbands. In Fig. 6.9 the spin splitting, $\Delta \mathrm{E}=\mathrm{g}^{*} \mu_{\mathrm{B}} \mathrm{B}$, is plotted against magnetic field to investigate the variation with filling factor. It can be seen that the magnetic field dependence alone is not sufficient to account for the enhanced values of the $g$-factor in the various samples. Also in a given sample $\Delta \mathrm{E}$ increases linearly with $\mathrm{B}$ suggesting that in each case $\mathrm{g}^{*}$ does increase with magnetic field, but not very rapidly.

From Eqn. 6.9 there appear two ways in which the value of $\mathrm{g}^{*}$ could increase either by increasing the spin-population difference; or by having a larger exchange energy. However the problem must be solved self consistently as a larger exchange energy will result in a larger population difference. If $E_{e x}$ were the same in all samples then the population difference would just be fixed by the magnetic field. Since all these measurements are at odd integral filling factors below the magnetic quantum limit where only electrons of the last Landau level contribute to conduction, the same value of $\mathrm{g}^{*}$ should be measuread at a given magnetic field regardless of the carrier density. Fig. 6.9 shows that this is clearly not the case, and so it must be concluded that the exchange energy is greater at lower carrier concentrations.

The opposite behaviour might have been expected, since at higher $n_{s}$ there should be more overlap of the electronic wavefunctions and so a greater exchange energy. In a magnetic field the situation will be slightly different as the eigenstates of the system will be Landau levels and the wavefunctions in each level will be orthogonal. Therefore only the electrons in a given Landau level will contribute strongly to the exchange energy in that level. Thus at a given magnetic field and odd integral filling factor the total carrier concentration will not be important to first order. At low $n_{s}$ the Fermi wavevector will be smaller and the width of the 2DEG will be smaller. This means 
there will in fact be more overlap of the wavefunctions at low carrier concentrations and so the exchange energy will be greater.

As stated above $g^{*}$ depends on the exchange energy and this will be larger in high mobility samples than in disorded ones where the correlations are weakened, which explains why such high values are found here. The mobility variation of $E_{e x}$ may be similar to the quantum life time, $\tau_{s}$, as it depends on the overlap of electronic wavefunctions, but $\tau_{s}$ is calculated from a single particle scattering picture rather than including the many body effects expected to be important for $g^{*}$. It will be seen in Chapter 7 that $\tau_{s}$ shows little variation between most of the samples considered here, but is always much larger in the upper subband. This may be in some way related, but the exact mechanism is not clear.

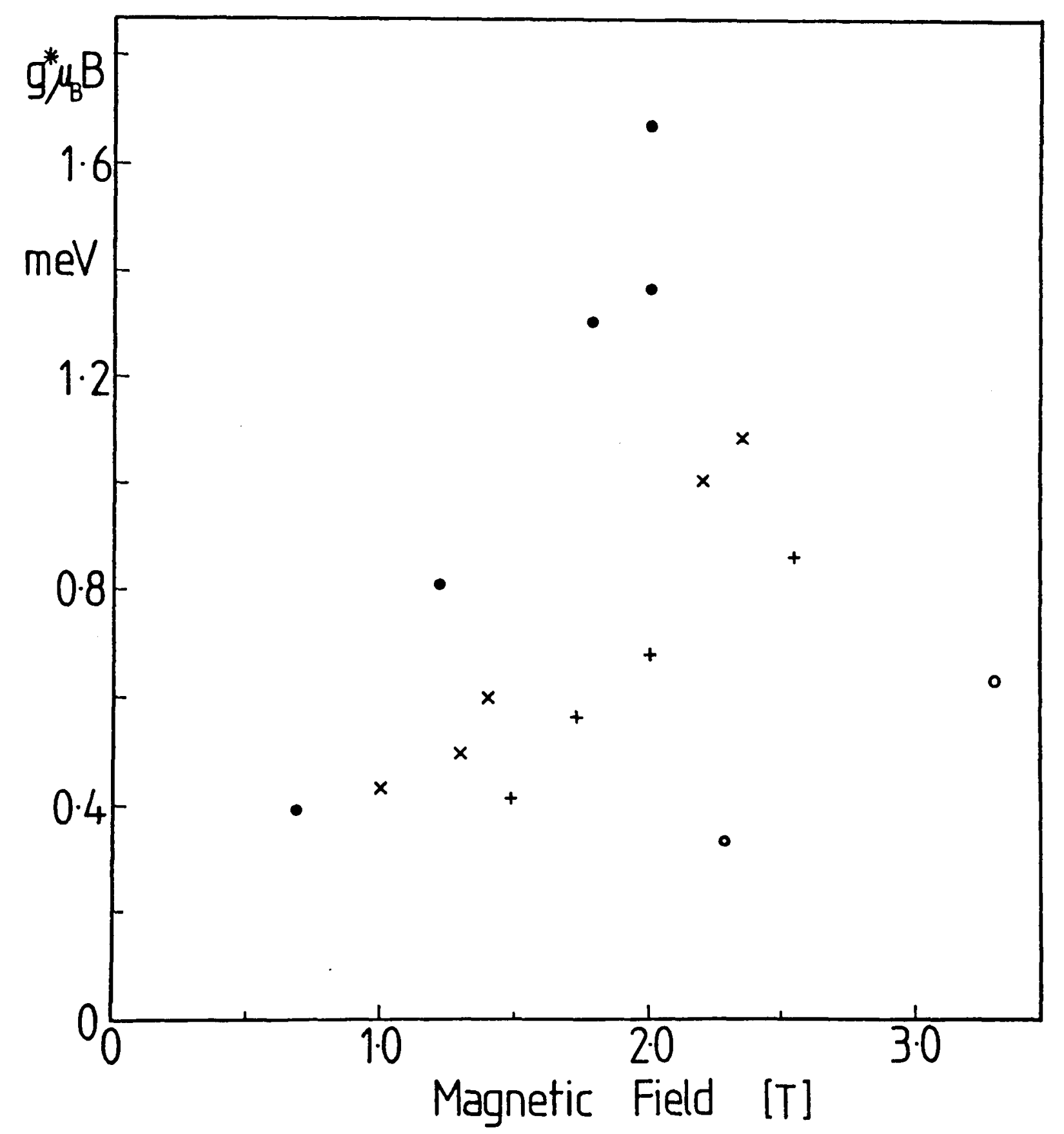

Figure 6.9 Spin splitting, $\Delta \mathrm{E}=\mathrm{g}^{*} \mu_{\mathrm{B}} \mathrm{B}$, as a function of magnetic field for several samples of different carrier concentrations: - $-G 215, \mathrm{n}_{\mathrm{s}}=5.5 \times 10^{10} \mathrm{~cm}^{-2}\left(\mathrm{E}_{1}\right.$ subband); - - G215, $7.2 \times 10^{11} \mathrm{~cm}^{-2}$ (E $E_{0}$ subband); $\times$ - G156, $1.7 \times 10^{111} \mathrm{~cm}^{-2}$; + - G104 $5.4 \times 10^{11} \mathrm{~cm}^{-2}$. 


\subsection{MAGNETORESISTANCE MEASUREMENTS IN PARALLEL MAGNETIC FIELDS}

\subsubsection{Depopulation of Subbands}

In Section 1.3.2 the energy states of a $2 \mathrm{DEG}$ in a magnetic field with components both perpendicular and parallel to the layers was shown to be

$$
E_{N, i}=\left(N+\frac{1}{2}\right) \frac{\hbar e B_{\perp}}{m^{*}}+E_{i}+\frac{e^{2} B_{\| 1}^{2}}{2 m^{*}}\langle\Delta z\rangle_{1}^{2}
$$

where $\langle\Delta z\rangle$ is the spread of the wavefunction perpendicular to the interface in the $i^{\text {th }}$ subband, which can be estimated as $200-400 \AA$ (Stern \& Howard 1967, Ando 1982, Stern \& Das Sarma 1984). The last term represents a positive diamagnetic shift in energy of each subband on application of a parallel field component giving a change in subband separation of :-

$$
\begin{aligned}
\Delta\left(E_{1}-E_{0}\right) & =\frac{e^{2} B_{\|}^{2}}{2 m^{*}}\left[\langle\Delta z\rangle_{1}^{2}-\langle\Delta z\rangle_{0}^{2}\right] \\
& \propto B_{\| 1}^{2}
\end{aligned}
$$

and as the spread of the higher subband wavefunction will be greater than that of the lower subband, $E_{01}$ will increase with parallel field. This diamagnetic shift was first detected by Beinvogl et al. (1976) from shifts in the resonance position of the $0 \rightarrow 1$ inter-subband transition in a FIR transmission experiment on $\mathrm{Si}$ surface charge accumulation layers.

On increasing $B_{\|}$there will be some field where $E_{01}>E_{F}$, and electrons are transfered to lower subbands. If $B_{\perp}=0$ then there will be a continuum of states in each subband and the density of states at the Fermi level will just depend on the number of accessible subbands. Depopulating a subband will give a decrease in resistivity, which is due to closing the inter-subband scattering channel. (This is not due to the absence of electrons in the upper subband as these have a much lower Fermi velocity than those in the lower subband $-v_{F} \propto n_{s}^{1 / 2}-$ so will never have made a great contribution to $\rho_{x x}$.) Englert et al. (1983) observed such a decrease in resistivity in a GaAs-GaAlAs heterojunction and from the mobility calculated either side of the depopulation event deduced that inter-subband scattering reduces the carrier 
mobility by as much as $50 \%$.

The value of magnetic field at which this depopulation occurs, $B_{d p}$, is a measure of the upper subband population, $n_{1}$, since the higher the Fermi level is in $E_{1}$ the greater the field that will be required to depopulate the subband. The difference between $E_{F}$ and the bottom of the $E_{1}$ subband, $E_{F 1}$, is given by Eqn. 6.1 with $n_{s}$ replaced by $n_{1}$, but depopulation will not be complete until $\Delta \mathrm{E}$ is twice this value since $\mathrm{E}_{\mathrm{F} 0}$ rises by an amount $E_{F 1}$ when the $n_{1}$ electrons are transferred to the lower subband.

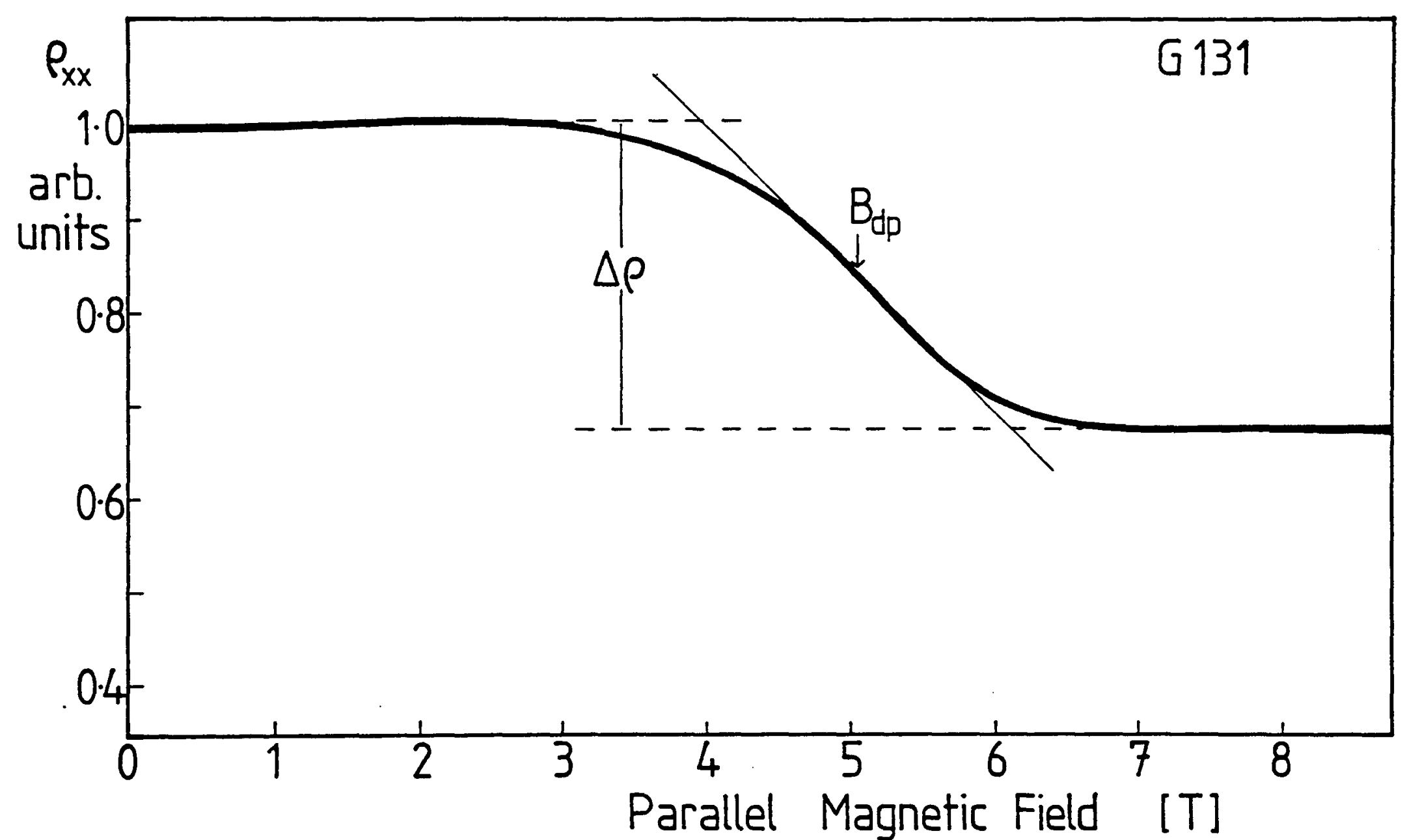

Figure 6.10 Depopulation of a subband by application of parallel magnetic field in a GaAs-GaAlAs heterojunction, shown by the sharp drop in resistivity.

A typical experimental recording showing subband depopulation is given in Fig. 6.10 for Sample G131. The drop in resistivity is not a sharp step due to broadening of the upper subband edge, so $B_{d p}$ will be taken as the position of maximum negative gradient. Notice that $\rho_{\mathbf{x x}}$ is constant for $B<B_{d p}$, in contrast to measurements on GaInAs-InP (results for S52a are shown in Fig. 6.11, also Razeghi et al. 1986), GaInAs-AlInAs (Portal et al. 1982, Newson et al. 1986) and $\mathrm{HgCdTe}$ (Singleton et al. 1986) where a significant increase in resistivity is seen just before depopulation. A magnetic field in the plane of the 2DEG has the effect of compressing the electronic wavefunction closer 


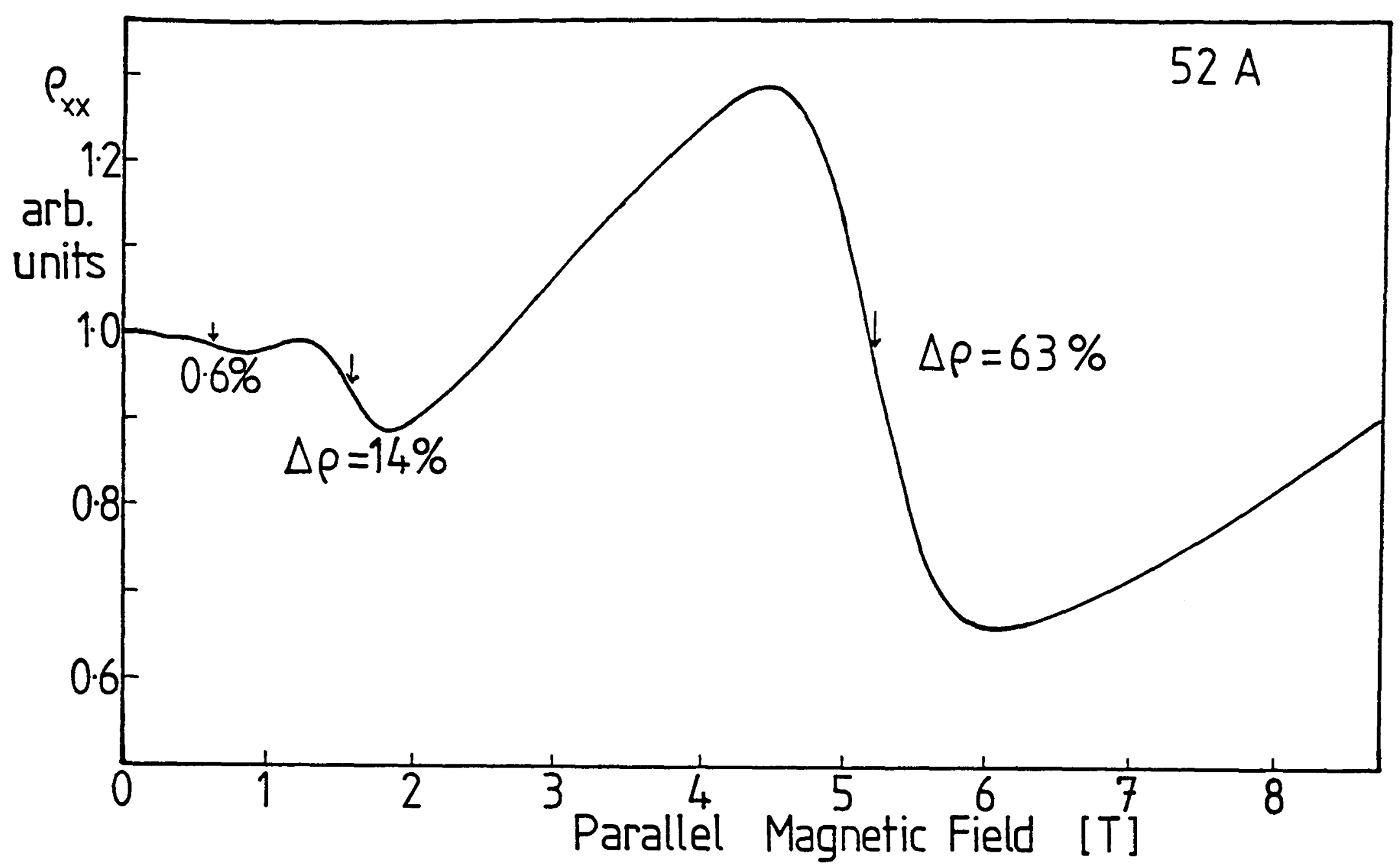

Figure 6.11 Parallel field depopulation of a GaInAs-InP heterojunction showing four subbands.

to the interface, making interface roughness scattering more important at higher fields. That it is absent in GaAs-GaAlAs is evidence of a much smoother interface than in GaInAs-InP. Above $B_{d p}$ there is again a much smaller increase in $\rho_{x x}$ than found in other systems, which varies between samples - suggesting variations in interface quality.

I have studied the magnetoresistance of Sample G131 in parallel fields, as a function of electron concentration, by use of the persistent photoconductivity induced by a red LED. Harris et al. (1987) extended these studies in similar samples to compare the effects of illumination by red and infrared diodes. It is worth noting that there was evidence of upper subband population from these parallel field measurements for much smaller $\mathrm{n}_{s}$ than was required to see Shubnikov-deHaas oscillations from the upper subband and at a similar point that peak asymmetry became apparant at high perpendicular fields.

The variation of $B_{d p}$ with upper subband population is shown in Fig. 6.12 from which the empirical law:

$$
\mathrm{B}_{\mathrm{dp}}=2.4 \times 10^{-6} \mathrm{n}_{1}^{0.6}
$$




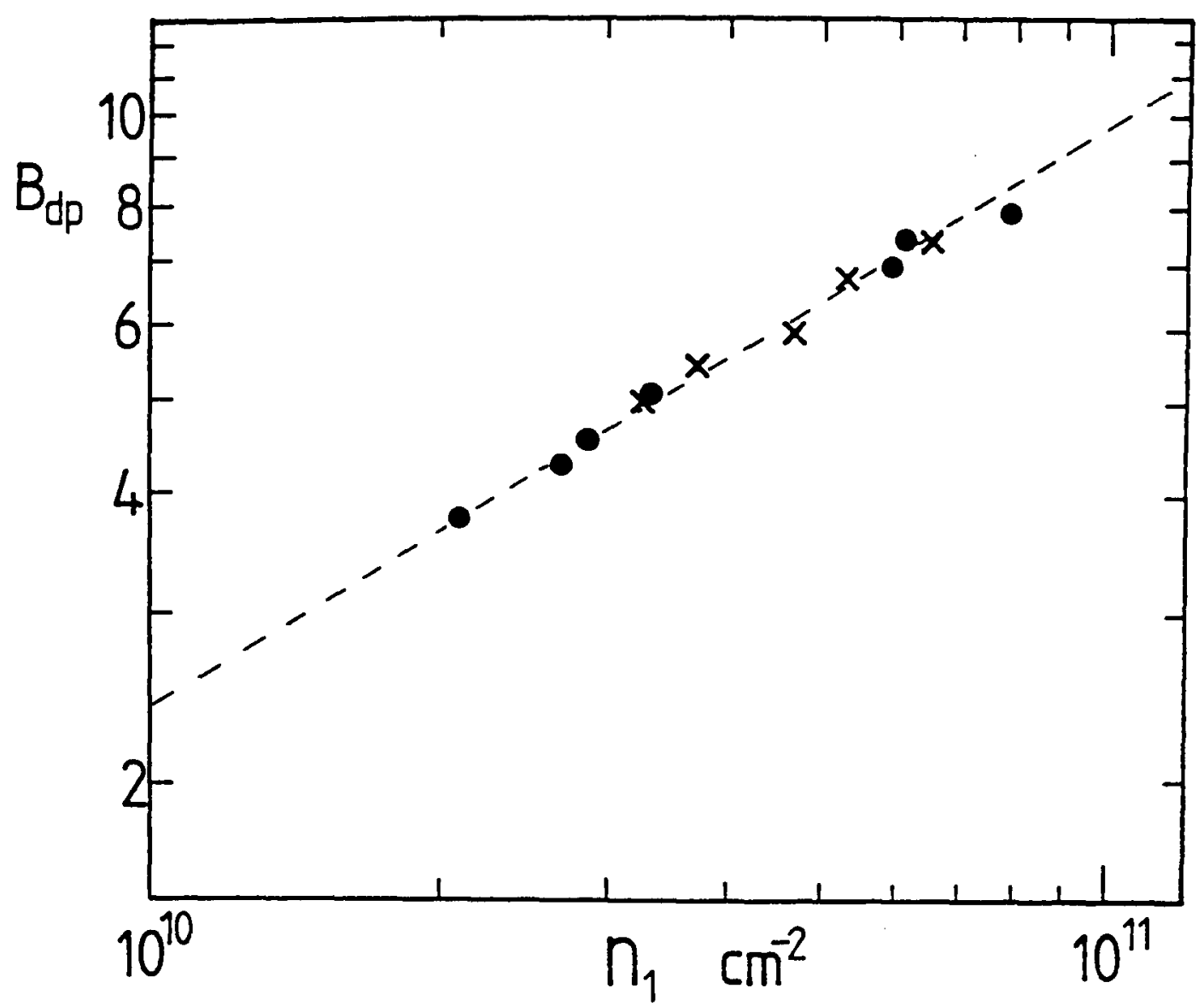

Figure 6.12 Variation of the parallel field required to depopulate the upper subband of G131 with upper subband population.

is deduced, with $\mathrm{n}_{1}$ in $\mathrm{cm}^{-2}$. This carrier concentration dependence is in reasonable agreement with Harris et al. who find an exponent of $2 / 3$, but not with the variational calculations (Ando 1982, Walukiewicz et al. 1984, Newson et al. 1986) which find the extent of the wavefunction scaling like $n^{-1 / 3}$ (cf. Eqn. 1.10) and hence $B_{d p} \propto n^{5 / 6}$. Studies on other materials (Zhao et al. 1985, Nasir et al. 1988) find an almost linear dependence of $B_{d p}$ on $n_{s}$, however they ignore the finite value of $n_{c r}$, which is only a good approximation in narrow gap materials. In the present case a plot of $B_{d p}$ against . $n_{s}$ is also linear, but with an offset not equal to $n_{c r}$ (in fact from plots similar to Fig. 6.12 fits can be obtained to show $B_{d p} \propto n_{s}^{1.5}$, or $B_{d p} \propto\left(n_{s}-5.5 \times 10^{11}\right)^{0.6}$ as predicted by Eqns. $6.4 \& 6.13$ ). However, the range of the data is not sufficient to produce an accurate fit to a power law.

As the calculations are not material specific, Nasir et al. (1988) plotted results of depopulation experiments from $\mathrm{HgCdTe}$ together with those from GaInAs-InP, GaInAs-AllnAs and the present results, in suitably reduced units of $\tilde{\mathrm{N}}=\mathrm{n}_{s} / \mathrm{a}^{*}$ and $\gamma=\hbar \omega_{c} / R^{*}$, where $a^{*}$ is the effective Bohr radius and $R^{*}$ the effecive Rydberg. This is shown in Fig. 6.13 and displays an approximately 'universal' behaviour for all these quasi-accumulation layers, as originally predicted by Zhao et al. (1985). 


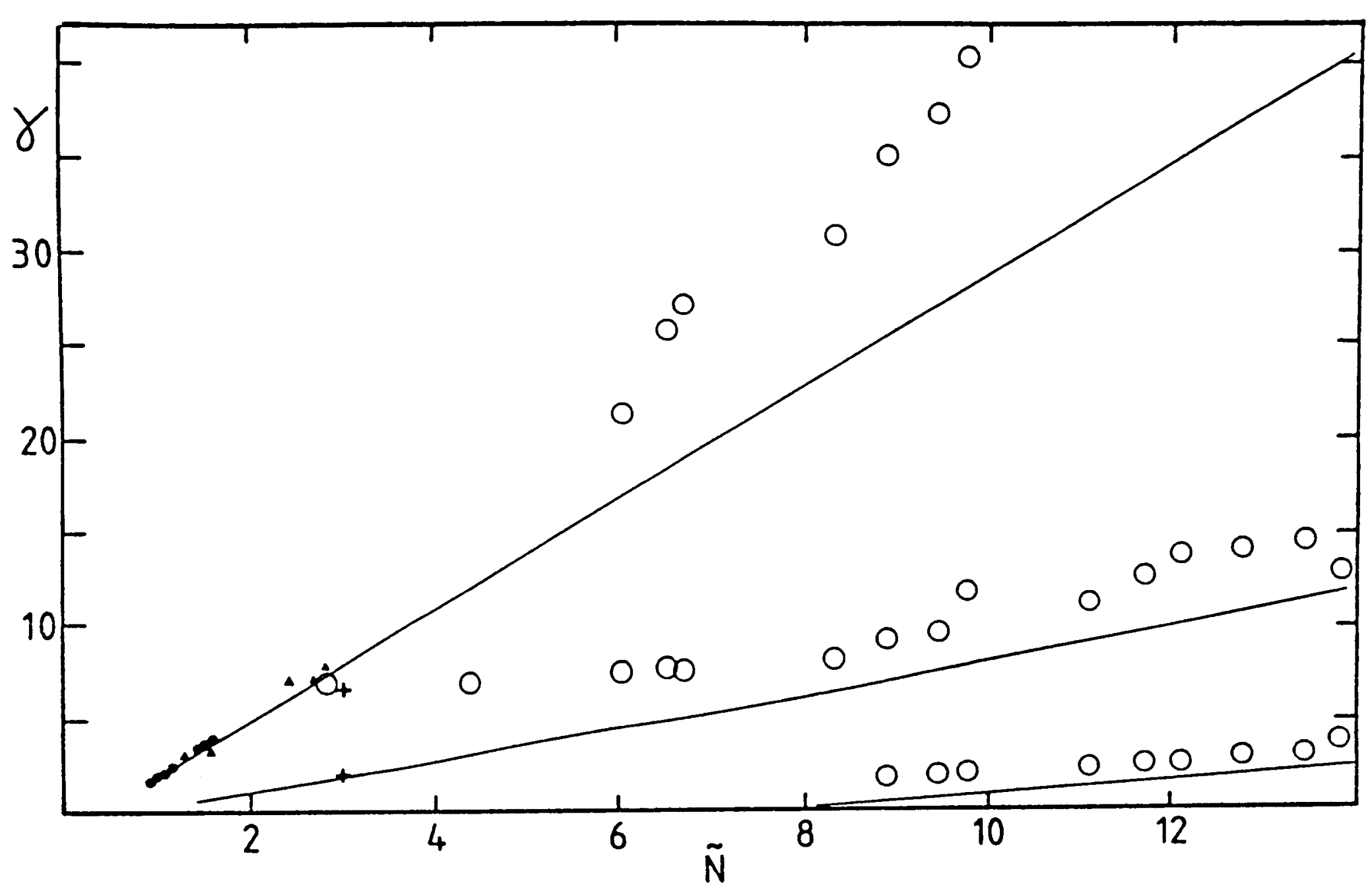

Figure 6.13 "Universal" plot of depopulation field against carrier concentration in reduced units for $2 \mathrm{D}$ systems.

- GaAs-GaAlAs (this work); O HgCdTe (Nasir et al.); + GaInAs-InP (Razeghi et al.); $\Delta$ GaInAs-InAlAs (Newson et al.); lines show the model of Reisinger \& Koch.

\subsubsection{Measurement of Inter-Subband Scattering Rate}

From the data on G131 it was also possible to measure how the change of mobility . on depopulation varied with $n_{s}$. Figure 6.14 demonstrates a linear dependence of $\Delta \mu / \mu$ with total carrier concentration showing a maximum observed change of $43 \%$. (Similar plots against $n_{1}$ or $B_{d p}$ are also linear but do not pass through the origin.) When one also considers Fig. 6.2, from which the increase in zero-field mobility with $n_{s}$ is seen to be approximately linear in the region of interest, it appears that Fig. 6.14 really shows the variation in intra-subband scattering from the lowest subband, with a constant inter-subband scattering rate. This suggestion was investigated by measuring the mobility both at $B=0$ and above the depopulation, to arrive at scattering rates $\left(\tau^{-1}=\mathrm{e} / \mathrm{m}^{*} \mu\right)$ which should differ by the inter-subband scattering rate ie.

$$
\frac{1}{\tau_{\text {total }}}=\frac{1}{\tau_{\text {intra- }}}+\frac{1}{\tau_{\text {inter- }}}
$$




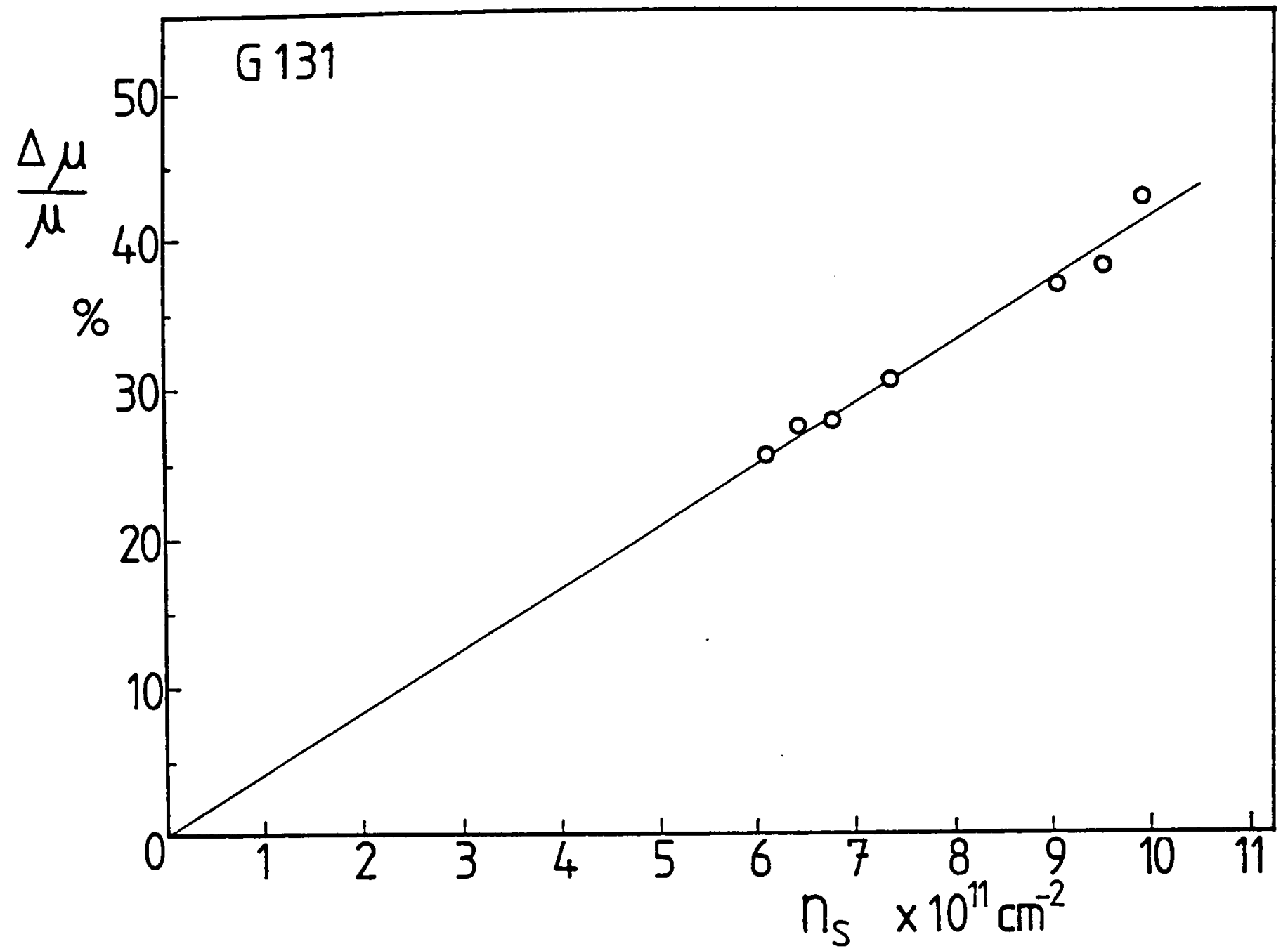

Figure 6.14 Relative change of mobility on depopulation of the upper subband in G131 as a function of total carrier concentration.

Before presenting the results it is necassary to consider two possible pitfalls: contributions to the mobility from upper subband electrons and magnetic field variation of the scattering rates. In the upper subband both $n_{s}$ and $k_{F}$ are much smaller than in the lower subband so there will be a minimal contribution to $\mu$. Secondly, as seen in Fig. 6.10, the resistance is constant both above and below the depopulation event,. showing the scattering rates do not depend on magnetic field parallel to the interface.

The constant value of $\tau_{\text {inter- }}{ }^{-1}$ found in Fig. 6.15 clearly demonstrates that inter-subband scattering switches on rapidly on population of the upper subband and that it is then independent of carrier concentration. This striking result may be explained by the constant density of available final states in the upper subband, with the shape of the initial increase being determined by the broadening of the subband edge. A more detailed study could be used to map out the DOS in the upper subband. Meanwhile, the intra-subband scattering decreases with increasing $n_{s}$ due to the increased wavevectors of the electrons, so that by the highest carrier concentrations studied the scattering rates from the two processes are comparable. 


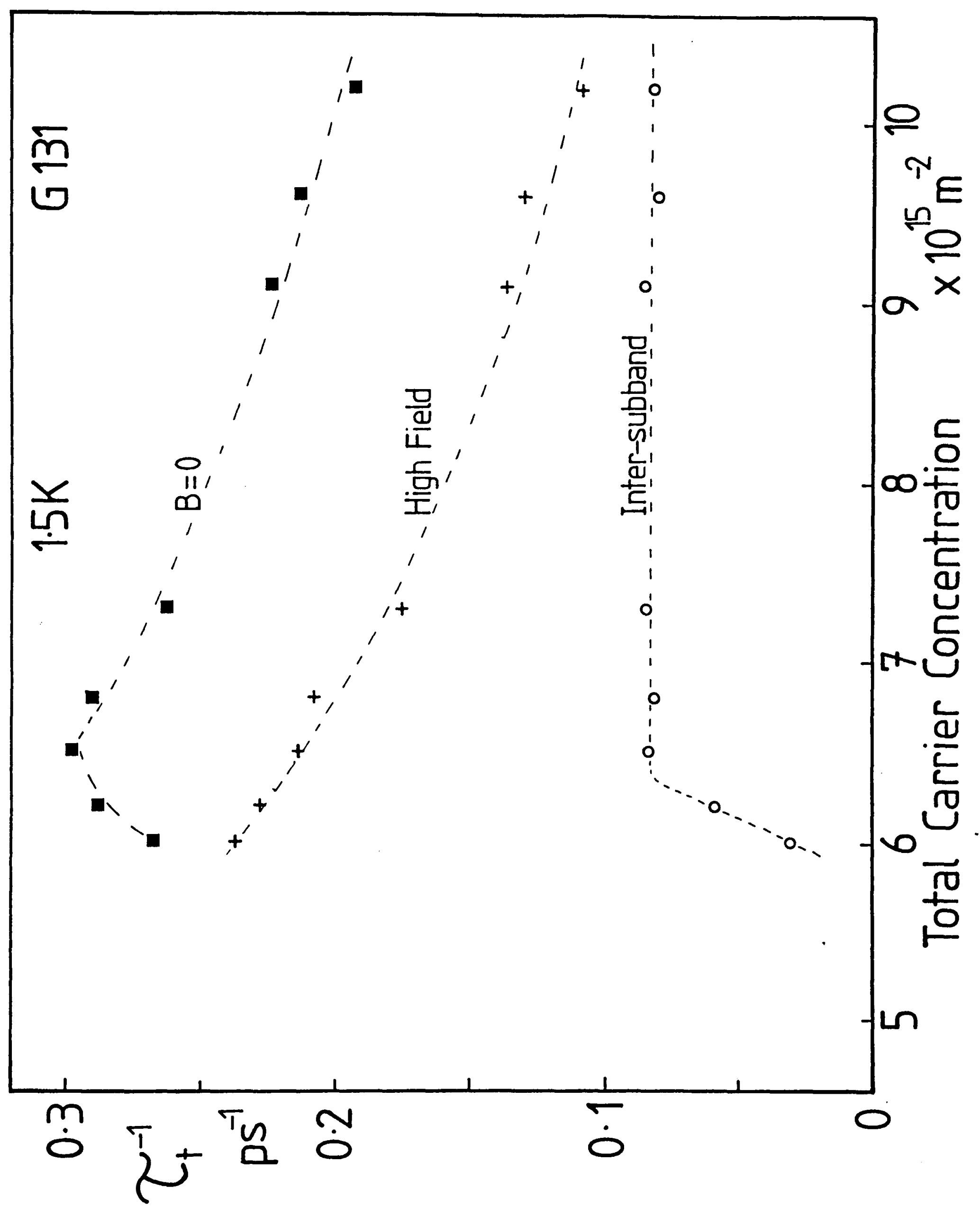

Figure 6.15 Scattering rates for electrons below $(\boldsymbol{\square})$ and above $(+)$ a depoplation event, from which an inter-subband scattering rate $(0)$ is deduced. 


\subsection{EFFECTS OF INTER-SUBBAND SCATTERING ON SdH OSCILLATIONS}

\subsubsection{Modulation of the Oscillations at Increased Temperatures}

On warming the lattice the Shubnikov-deHaas oscillation amplitude usually decreases in accordance with

$$
\frac{\Delta \rho}{\rho} \propto \frac{\chi}{\sinh \chi}
$$

where $\chi=2 \pi^{2} \mathrm{kT} / \hbar \omega_{\mathrm{c}}$ as discussed in Section 2.2.

\section{Increased Lattice Temperatures}

However, when measurements are made on samples with two populated subbands at temperatures above $1 \mathrm{~K}$, the low field oscillations begin to change their character, as shown in Fig. 6.16 (top trace). The two series are no longer additive, and the amplitude of the higher frequency series, associated with the movement of $E_{F}$ through the $E_{0}$ subband, becomes modulated by the period of the oscillations from the upper subband. In particular it is worth noting that the phase of the modulation corresponds

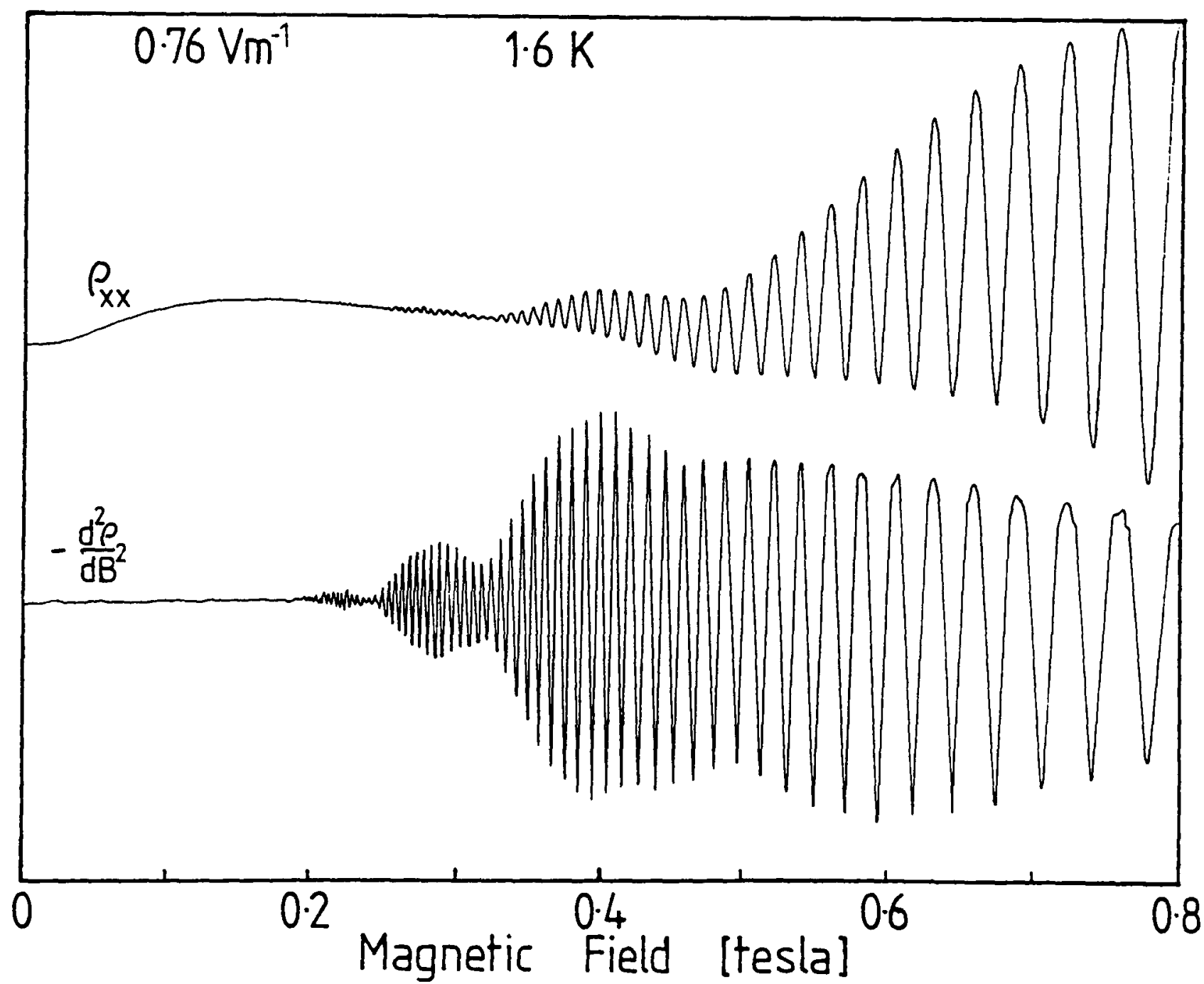

Figure 6.16 Shubnikov-deHaas oscillations from G215 with 2 populated subbands at $1.6 \mathrm{~K}$, showing a modulation of the amplitude of one series by the other at very low magnetic fields. Top trace is direct and the lower shows the second derivative where the beating is more obvious. 
to a minimum amplitude of the $E_{0}$ subband series at the minima of the oscillations from the upper subband. At the same time the overall oscillation amplitude decreases due to the conventional thermal damping described by Eqn. 6.15 , but the positions of the minima remain unchanged. The multiplicative effect is demonstrated very clearly when the oscillations are enhanced by the use of a second derivative, as shown in the lower trace. This suppresses the lower period series, due to its lower frequency, leaving a recording with an apparent beating effect caused by the amplitude modulation. By $4.2 \mathrm{~K}$ the thermal damping of the oscillations is sufficient to make most of the modulation effects too weak to detect, as can be seen in Fig. 6.17.

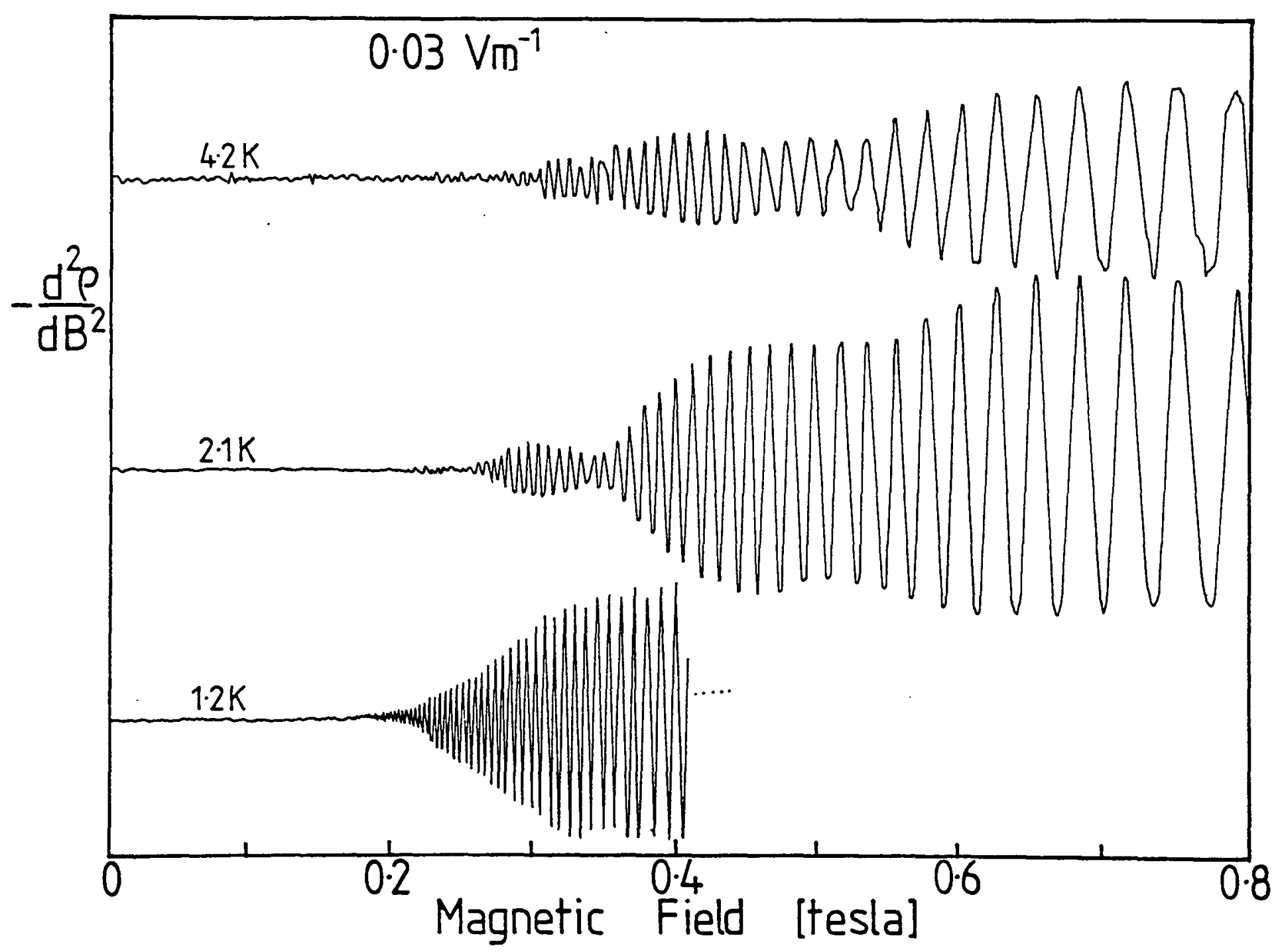

Figure 6.17 Second derivatives of the low field $\mathrm{SdH}$ showing the appearence of modulation as the temperature is increased.

\section{Increased Electron Temperature}

The modulation effect could also be made to re-appear at low temperatures by the application of an electric field in the plane of the 2DEG. The electric fields used had a negligible effect on the zero field resistivity, but was sufficient to heat the electron gas to temperatures of $1-3 \mathrm{~K}$, as shown in Fig. 6.18. The data at lower electric 


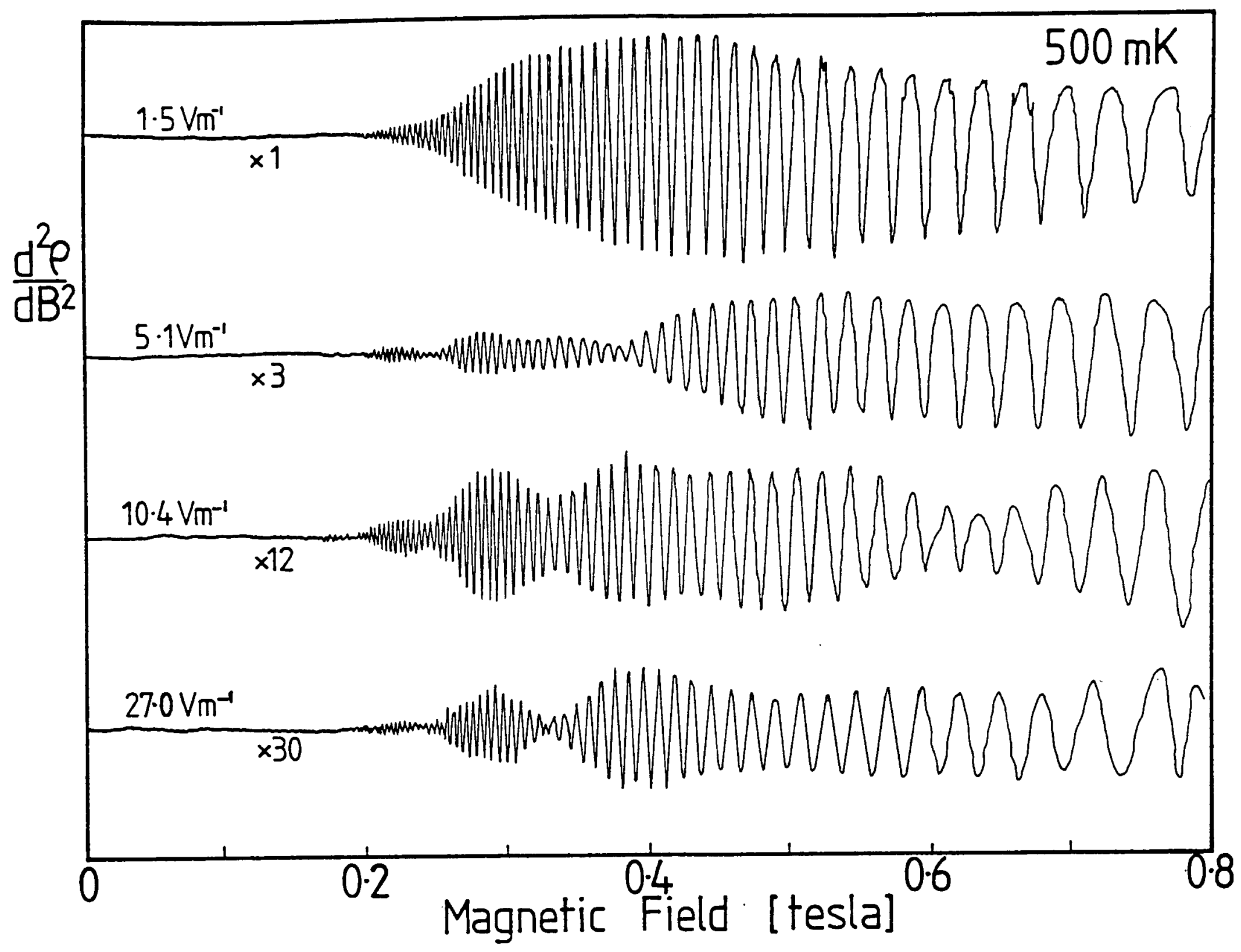

Figure 6.18 Second derivatives of low field magnetoresistance showing the effect of raising the electron temperature by application of an electric field at a lattice temperature of $500 \mathrm{mK}$. The multipliers indicate how the oscillation amplitudes decrease with increasing electron temperature.

fields are very similar to those shown in Fig. 6.16, however above $10 \mathrm{Vm}^{-1}$ significant differences begin to occur, particularly at higher fields (the steady decrease in amplitude above $0.4 \mathrm{~T}$ is a consequence of the frequency response of the differentiators). This is probably associated with the magnetic field dependence of the electron energy loss rate, and will be discussed more fully in Chapter 8. It appears that raising the electron temperature in this fashion causes more pronounced beats than by warming the lattice, but does not affect the minimum magnetic field at which oscillations may be observed. This last observation is evidence that Landau level width is only sensitive to lattice temperature (not electron temperature), which will be the case for acoustic phonon limited broadening.

The modulation of the two series could be seen in all samples studied when two 
subbands were occupied, and did not seem to be a strong function of the mobility. This is illustrated in Fig. 6.19, where the modulation effect is shown in both $G 131$ and G215 with respective mobilities of 12 and $67 \mathrm{~m}^{2} \mathrm{~V}^{-1} \mathrm{~s}^{-1}$ and similar electron concentrations $\left(10 \& 8 \times 10^{11} \mathrm{~cm}^{-2}\right)$. Although the oscillations continue to lower field in the higher mobility sample the magnitude of the modulation is very similar in both cases. It should be remarked that although the d.c. mobilities differ by a factor of 5 the Dingle Temperatures differ between the samples by less than a factor of 2 in the $E_{0}$ subbands and only by $\sim 30 \%$ in the upper subband. This suggests the modulation is more sensetive to the single particle relaxation time than the zero field momentum relaxation time.

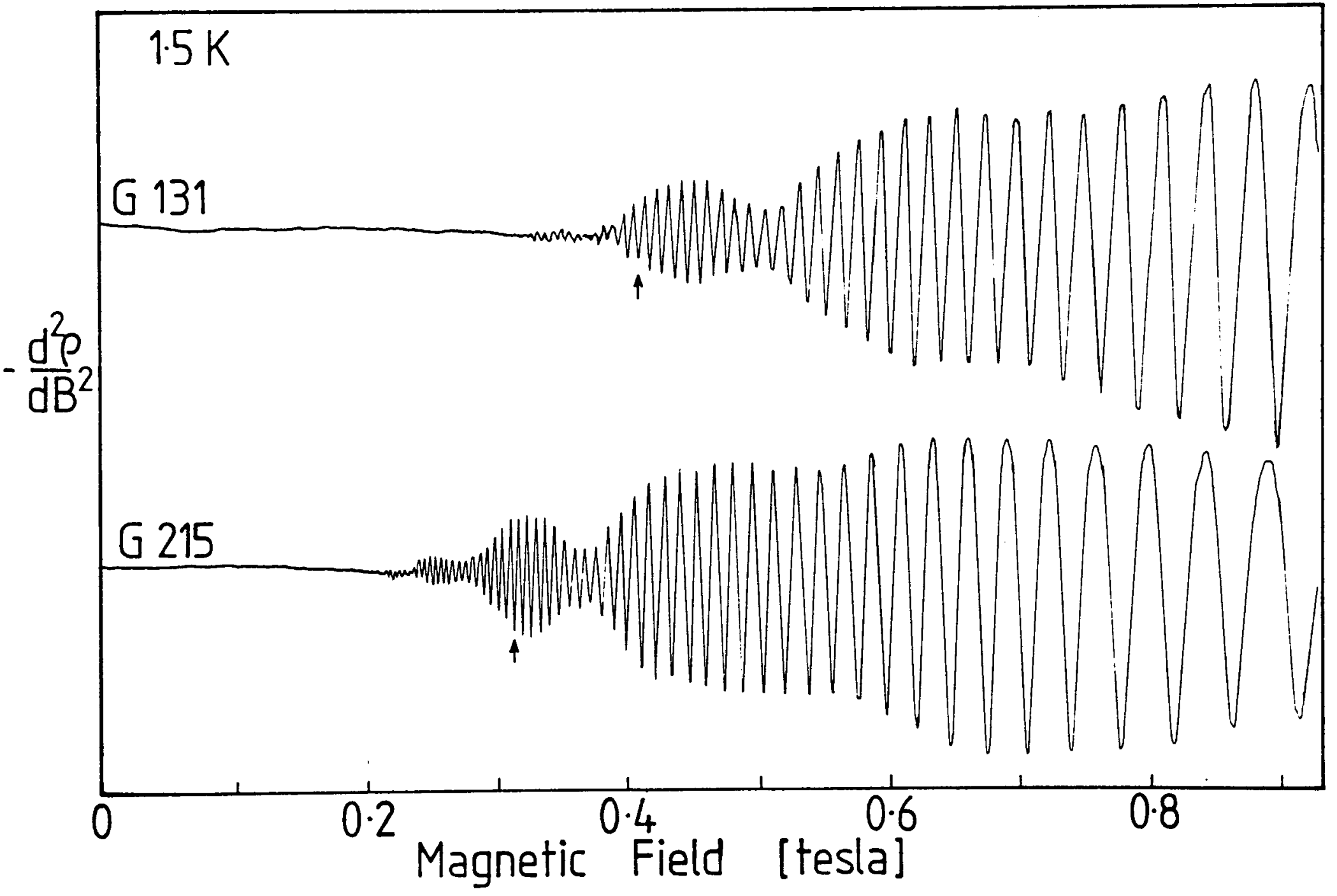

Figure 6.19 The modulation effect shown in a high mobitity (G215) and lower mobility (G131) sample. The arrows indicate oscillations of Landau index 50 .

\subsubsection{Explanation of the Modulation}

The preceeding section has described a dramatic change in the structure of Shubnikov-deHaas oscillations from samples containing more than one occupied subband. 
In discussing the origin of these phenomena a number of factors need to be born in mind:

(i) The appearance of the modulation requires the population of the second subband. No evidence of this effect was seen in these samples before the population of the second subband, nor has any been reported previously.

(ii) Temperature is clearly important, suggesting that phonon scattering may play a role.

(iii) The maximum amplitude of the $E_{0}$ series corresponds to a maximum in the resistivity of the $E_{1}$ series. This is the condition for resonant (elastic) inter-subband scattering, so the oscillations have not been suppressed by the increased scattering.

These factors suggest two possible mechanisms for the the appearance of the modulation effect:

(a) Oscillatory screening of the scattering mechanisms caused by the increased density of states when the Fermi level lies in the middle of a Landau level of the upper subband.

(b) Oscillations of the inter-subband scattering rate which lead to non-linear contributions to the total conductivity in a magnetic field.

Both processes will probably occur to some extent, however the second explanation is favoured as the main cause, since (a) would require an oscillatory screening of the acoustic phonon scattering as well as a very strong modulation of the damping. Values of the Landau level broadening deduced from $\tau_{s}$ in Section 6.3 imply only a small variation in the density of states at $E_{F}$ and any oscillatory screening, which is a second order effect, would thus be too small to acount for the observed modulations.

In more detail (b) can be shown to occur by using the diffusion picture for conductivity in high magnetic fields (Aoki 1987). In this case the conductivity $\left(\sigma_{\mathbf{x x}}\right)$ is given by

$$
\sigma_{x x} \approx e^{2} \mathrm{D}\left(E_{F}\right) D^{*}
$$


where $D\left(E_{F}\right)$ is the density of states at the Fermi energy and $D^{*}$ is the diffusion constant, given approximately by

$$
D^{*} \approx 1_{N} 2 / \tau
$$

where $1_{N}$ is the cyclotron radius of the appropriate Landau level, and $\tau$ is the scattering time which will also oscillate. For any number of occupied subbands we may write

$$
\sigma_{x x} \approx e^{2} \sum_{i j} D_{i}\left(E_{F}\right) \quad l_{N}^{i} 1_{N}^{j} / \tau_{i j}
$$

which will generate both independent $(i=j)$ and modulated $(i \neq j)$ series of oscillations, coming from the intra- $\left(1 / \tau_{\mathrm{ii}}\right)$ and inter $-\left(1 / \tau_{\mathrm{ij}}\right)$ subband scattering. At low fields we may approximate $D\left(E_{F}\right)$ and $1 / \tau$ to a $\sin ^{2}$ variation with field, with a frequency $\nu_{i}=n_{i} h / 2 e B$ where $n_{i}$ is the carrier concentration in that subband. Thus we may write

$$
\Delta \sigma_{\mathrm{xx}} \approx \sum_{\mathrm{ij}} \mathrm{A}_{\mathrm{ij}} \sin ^{2} \pi \nu_{\mathrm{i}} \sin ^{2} \pi v_{\mathrm{j}}
$$

where the coefficients $A_{i j}$ are related to the strength of the intra- $(i=j)$ and inter- $(i \neq j)$ subband scattering.

For the two subband systems here:-

$$
\Delta \sigma_{\mathrm{xx}} \approx \mathrm{A}_{00} \sin ^{4} \pi v_{0}+\mathrm{A}_{11} \sin ^{4} \pi v_{1}+2 \mathrm{~A}_{01} \sin ^{2} \pi v_{0} \sin ^{2} \pi v_{1}
$$

Using this formalism the results now have a ready explanation. At very low temperatures the inter-subband scattering is completely suppressed $\left(A_{01}=0\right)$, and there are two additive series of oscillations. By $\mathrm{T} \sim 1.5 \mathrm{~K}$, the inter-subband scattering has appeared, and the modulation now becomes significant due to its apparently dominant influence on the conductivity. This requires us to assume (i) that in this temperature range the dominant inter-subband scattering mechanism is by acoustic phonons, and (ii) that this process can also dominate the normal intra-subband scattering.

The considerable relative importance of inter-subband scattering required by the second assumption was discussed in Section 6.5, where changes in resistivity of a factor of two were found when the inter-subband scattering was suppressed. 


\subsubsection{Acoustic Phonon Mediated Inter-Subband Scattering}

Let us now consider the temperature dependence of the inter-subband scattering. There is a considerable difference between the wavevectors of electrons at the Fermi level in the two subbands, illustrated schematically in Fig. 6.20, and inter-subband transitions will only occur if this change in wavevector $(\Delta \mathbf{k})$ can be accounted for. Acoustic phonons could provide the appropriate momentum transfer. The minimum requirement would be for a phonon of wavevector $\Delta \mathbf{k}$ and energy $E_{\min }$ given by :-

$$
\Delta \mathbf{k}=\mathbf{k}_{\mathrm{F} 0}-\mathbf{k}_{\mathrm{F} 1}
$$

$\&$

$$
E_{\min }=\hbar v_{s} \Delta \mathbf{k}
$$

The Fermi wavevector in the $i^{\text {th }}$ subband is $k_{F i}=\left(2 \pi n_{i}\right)^{1 / 2}$ where $v_{s}$ is the appropriate sound velocity

$$
\begin{aligned}
\therefore \quad \mathrm{E}_{\min } & =\hbar \mathrm{v}_{\mathrm{s}} \sqrt{2 \pi}\left(\overline{\mathfrak{n}}_{\mathrm{o}}-\sqrt{\mathrm{n}_{1}}\right) \\
& \sim 6.1 \mathrm{~K}
\end{aligned}
$$

where a value of $5000 \mathrm{~ms}^{-1}$ has been used for the velocity of sound in GaAs (Vass 1987) and the carrier densities are from the SdH measurements.

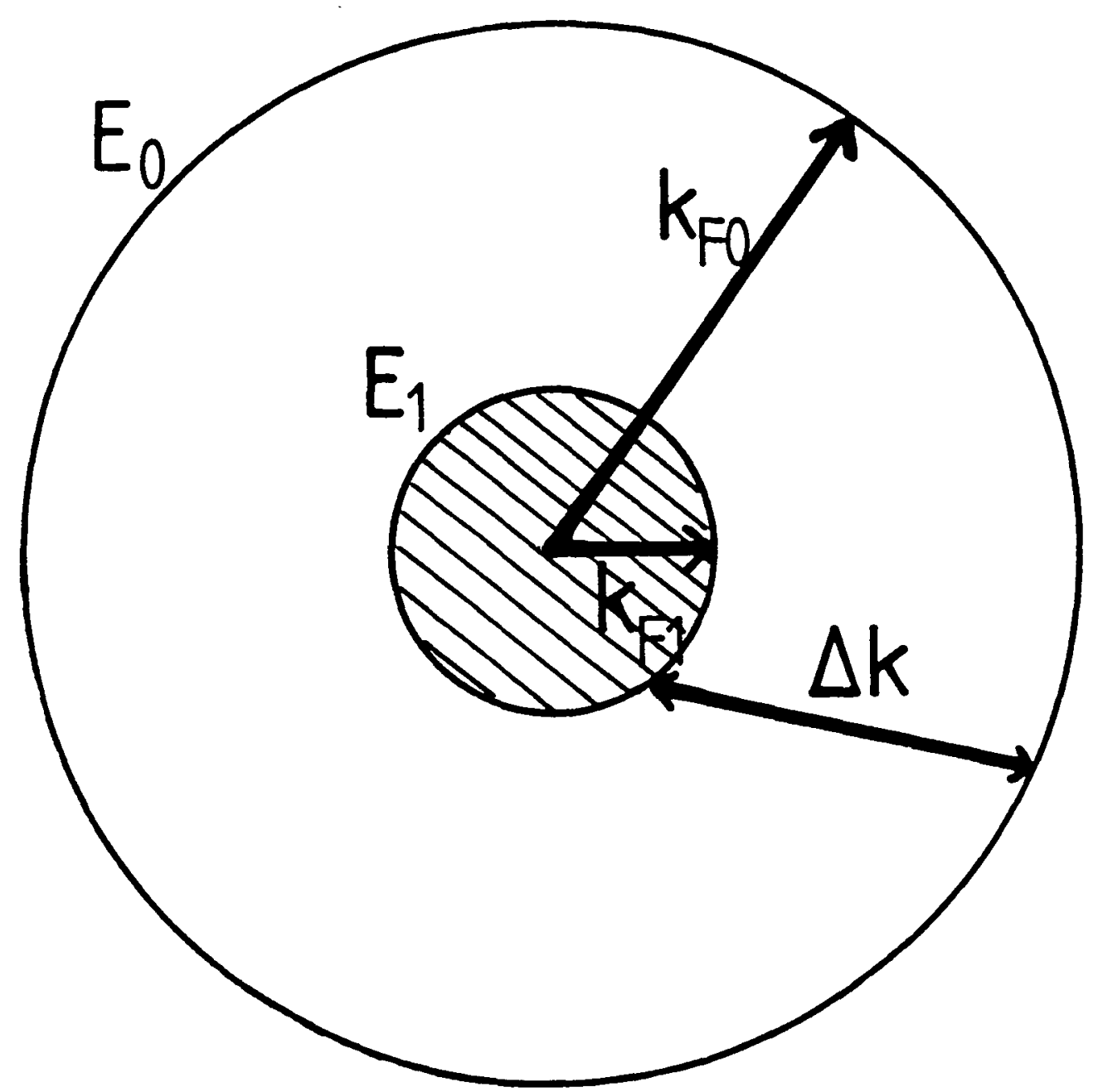

Figure 6.20 Schematic diagram showing wavevectors at the Fermi level in the $E_{0}$ and $E_{1}$ subbands and the required wavevector change for an inter-subband transition, $\mathbf{\Delta k}$. 
Taking account of the distribution of phonon modes, which Challis et al. (1987) found to be peaked at an energy $\sim 4 \mathrm{kT}$ (the value of the numerical factor is determined by the temperature dependence of the phonon scattering), it can be seen that acoustic phonon assisted inter-subband scattering will occur for $T \gtrsim 1.5 \mathrm{~K}$ and that cooling to $500 \mathrm{mK}$ will be sufficient to inhibit this process. (If the distribution of acoustic phonon modes is given by a Boltzman factor, $\exp (-\hbar \omega / \mathbf{k T})$, the population of $6 \mathrm{~K}$ phonons will decrease by four orders of magnitude between $2 \mathrm{~K}$ and $0.5 \mathrm{~K}$.)

At these lower lattice temperatures the mechanism can be re-activated by raising the electron temperature with the application of an electric field, when acoustic phonon emission processes will become important.

Our conclusion is therefore that inter-subband scattering can have a very strong influence on the magnetotransport in multiple subband systems, and that this causes non-additive contributions to the conductivity at temperatures such that acoustic phonon mediated scattering can still occur. Measurements on GaInAs-InP heterojunctions demonstrate that this effect may occur in other systems as shown in Fig. 6.21 for S52a (also seen by Kane et al. 1988).

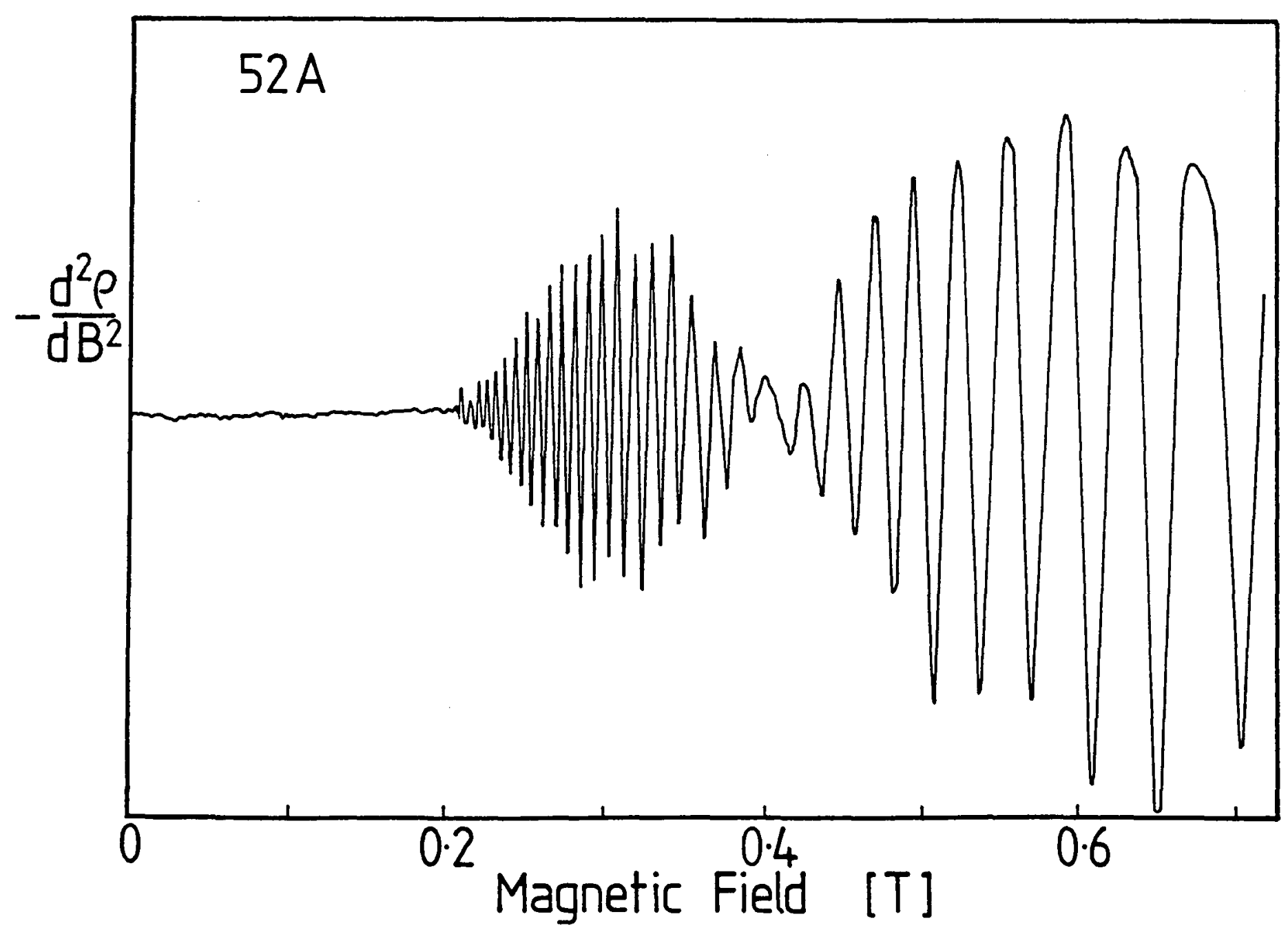

Figure 6.21 Strong modulation seen in a GaInAs-InP heterojunction at $1.6 \mathrm{~K}$ (Second derivative shown). 


\subsection{SUMMARY}

In this chapter magnetoresistance measurements at low temperatures have been used to study the principle scattering mechanisms in GaAs-GaAlAs heterojunctions. Inter-subband scattering has also been studied and found to have a very strong influence on the magnetotransport in multiple subband systems.

By depopulating the higher subbands with a magnetic field parallel to the 2DEG the changes in reistance of a factor of two were found due to suppression of the inter-subband scattering. Systematic studies as a function of carrier density show that opening of the inter-subband scattering channel to electrons of the lower subband has a greater effect on the mobility than the actual population of the upper subband and that the inter-subband scattering rate is independent of carrier concentration.

In perpendicular magnetic fields both subbands affect the Shubnikov-deHaas oscillations, but non-additive contributions are seen at electron temperatures $\gtrsim 2 K$, where acoustic phonon mediated inter-subband scattering is comperable to the intra-subband scattering.

At higher lattice temperatures in the previous chapter inter-subband scattering was seen to influence the magnetoresistance under warm electron conditions. It provides a resonant cooling channel for electrons with insufficient energy to emit optic phonons.

The Shubnikov-deHaas oscillations have also been used to find values for the subband separation and the effective $g$-factors. It transpires that $\mathrm{g}^{*}$ is much larger in the upper subband being enhanced 32 times above the bare Landé g-factor. From a variety of samples $\mathrm{g}^{*}$ is found to be much larger for low carrier concentrations. 


\section{CHAPTER 7}

\section{QUANTUM AND CLASSICAL RELAXATION TIMES \\ - THEIR RELATION TO SCATTERING MECHANSIMS IN MULTISUBBAND GaAs-AlGaAs HETEROJUNCTIONS}

$\begin{array}{lll}7.1 & \text { Introduction to } \tau_{s} \text { and } \tau_{t} & 201\end{array}$

7.2 Measurement of the Quantum Lifetime 202

7.3 Upper Subband Transport Mobility 208

7.4 Other Sources of Low Field Magnetoresistance 212

$\begin{array}{lll}7.5 & \text { Summary } & 216\end{array}$ 


\subsection{INTRODUCTION TO $\tau_{s}$ AND $\tau_{\mathrm{t}}$}

The mobility of conduction electrons depends on the time taken for momentum relaxation. There will generally be a number of scattering mechanism affecting the electrons of the 2DEG, each characterised by a different scattering time and range of interaction (Walukiewicz et al. 1984, Harrang et al. 1985) and changing with magnetic field in a diffent manner. Any given experimental measurement will be sensetive to several of these mechanisms, but not necessarily to the same extent as another method. This means that each type of measurement eg. zero-field resistivity, Shubnikov-deHaas, cyclotron resonance etc., will deduce a different value of mobility (Paalanen et al. 1983), which has led to much confusion in the past.

In studies of transport theory there are two characteristic times - a single particle relaxation time $\tau_{s}$ and a scattering time $\tau_{\mathfrak{t}}$, which are discussed by Das Sarma \& Stern (1985) and Harrang et al. (1985). $\tau_{t}$ is related to the Drude conductivity by the usual equation:-

$$
\sigma=n e^{2} \tau_{t} / m^{*}
$$

and hence this is the time related to the 'classical' zero-field transport mobility. $\tau_{t}$ can be calculated by solving the Boltzman transport equation as:-

$$
1 / \tau_{\mathrm{t}}=\int \mathrm{P}_{\mathrm{q}, \mathrm{q}^{\cdot}}(1-\cos \theta) \mathrm{dq^{ \prime }}
$$

where $P_{q, q^{\prime}}$ is the probability of scattering from state $q$ to state $q^{\prime}$, and $\theta$ is the scattering angle. Thus, it can be seen that $\tau_{t}$ is relatively insensitive to small-angle scattering.

$\tau_{s}$ may be regarded as the 'quantum' lifetime - it is a measure of the time for which the electronic wavefunction may be defined even in the presence of scattering, and is related to the Landau level collision broadening by $\Gamma=\hbar / 2 \tau_{s}$ (Eqn. 6.8). As it is a dephasing time, all scattering events will contribute equally to $\tau_{S}$ so it will be calculable from:-

$$
1 / \tau_{s}=\int P_{q, q^{\prime}} d q^{\prime}
$$


If the scattering is isotropic and the averaged value of $\cos \theta$ vanishes the relaxation time and scattering times will be equal. However, in modulation doped systems scattering from remote ionised impurities is important, so the distance between the plane of impurities and the 2DEG will enter into the scattering cross-section. This will introduce a $\theta$ dependence and the scattering will be peaked in the forward direction, making $\tau_{t}>\tau_{s}$

Measurements of a transport lifetime will always require a current path through the whole sample, so must always give a value averaged throughout the sample, this will be determined by the inhomogeneous broadening. By contrast lifetimes measured from cyclotron resonance will only depend on the conditions within a cyclotron radius and so will measure the homogeneous broadening. This will be the maximum $\tau$ measurable in any part of the sample. For example Hopkins et al. (1988) have measured a mobility from the cyclotron resonance linewidth of $\sim 7 \times 10^{8} \mathrm{~V}^{-1} \mathrm{~s}^{-1} \mathrm{~cm}^{-2}$ in sample G139, where the d.c. mobility is three orders of magnitude smaller.

\subsection{MEASUREMENT OF THE QUANTUM LIFETIME}

In Chapter 2 the amplitude of Shubnikov-deHaas oscillations was given as:

$$
\frac{\Delta \rho}{\rho}=f\left(\omega_{c} \tau\right) \frac{\chi}{\sinh \chi} \exp \left(-\frac{\pi}{\omega_{c^{\top} s}}\right)
$$

where the form of $f\left(w_{c} \tau\right)$ was discussed.

Dingle (1952) observed that in metals decreasing $\tau_{s}$ had the same effect on the oscillations as an increase in temperature. This allows an effective sample temperature $T_{\text {eff }}=T+T_{D}$ to be defined, where $T_{D}$ is the Dingle temperature related to $\tau_{s}$ by

$$
\tau_{s}=\frac{\hbar}{2 \pi \mathrm{kT}}
$$

In GaAs $\mathrm{m}^{*}=0.068$ which makes $\chi=1.00 \mathrm{~T} / \mathrm{B}$ - demonstrating its inherent suitability for this type of study! So Eqn. 7.4 can be approximated by:

$$
\frac{\Delta \rho}{\rho}=f\left(\omega_{c} \tau\right) \quad \frac{T}{\bar{B}} \exp \left[-\frac{T_{\text {eff }}}{B}\right]
$$

thus $T_{\text {eff }}$, and hence $\tau_{s}$, can be obtained from a plot of $\ln (\Delta \rho / \rho$ B) against $1 / B$, such 


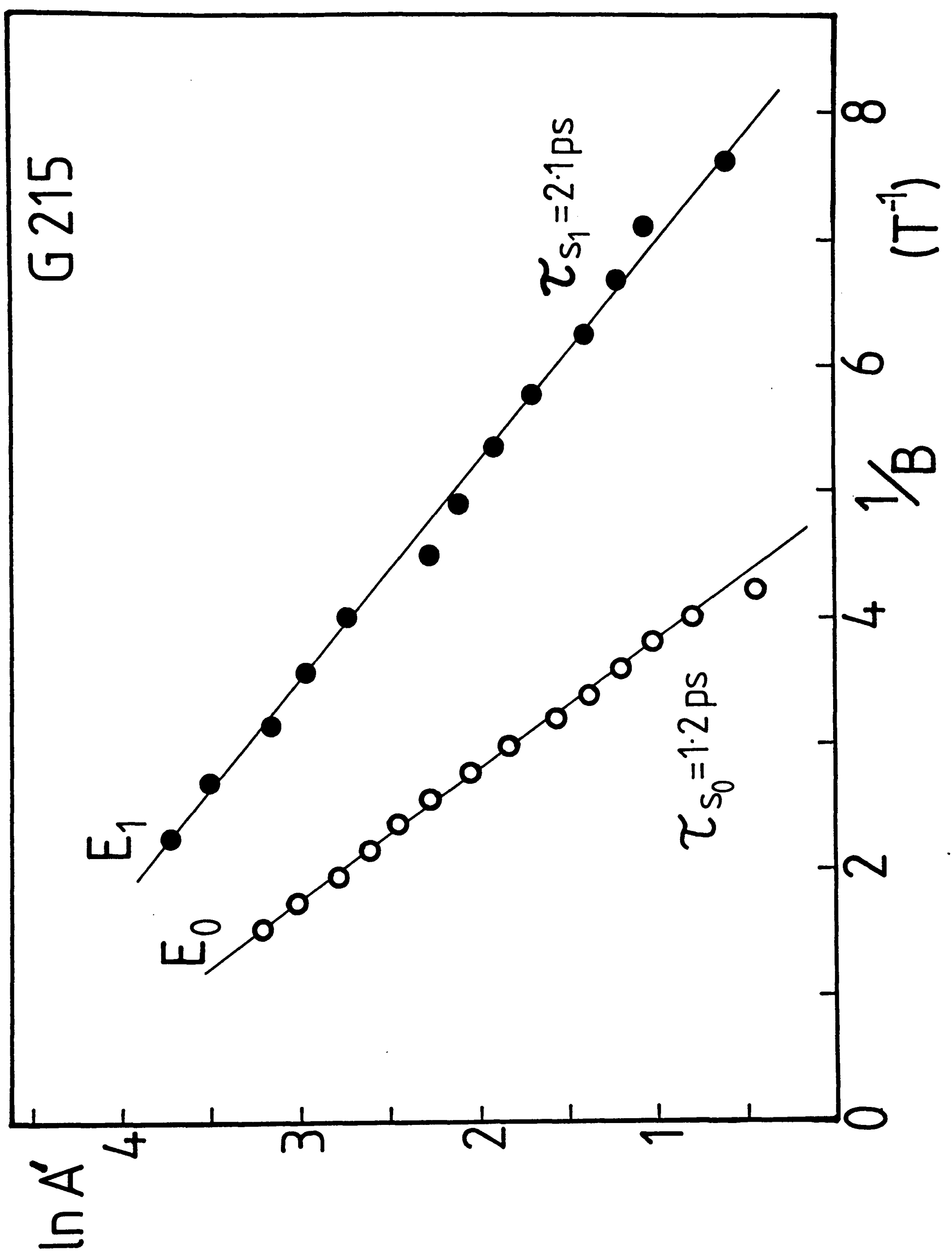

Figure 7.1 Plot of $\ln \mathrm{A}^{\prime}[=\ln (\mathrm{B} \Delta \rho / \rho)]$ against $1 / \mathrm{B}$, with arbitary origin, to determine the quantum lifetime $\left(\tau_{\mathrm{s}}\right)$ in each subband of G215. 
as Fig. 7.1 for sample G215 where the oscillations from both subbands have been considered. However, in all the work of Smith et al. (1988) $\ln \left(\Delta \rho / \rho B^{3}\right)$ has been used, which would only be the correct expression if conductivity oscillations were being observed, and leads them to deduce erroneously large values of $T_{D}$.

In the present case $\tau_{s}$ has been calculated using the full expression for $\chi / \sinh \chi$ both with and without $f\left(\omega_{c} \tau\right)$. If the time used in this prefactor is $\tau_{t}$ the results are indistinguisable from using a constant term and if $\tau_{s}$ is used (and calculated iteratively) they agree qualitatively but produce a $30 \%$ greater lifetime (Fletcher et al. 1988). The results are shown for a variety of samples at $500 \mathrm{mK}$ in Table 7.1 , and in Fig. 7.2 for G131 at $1.5 \mathrm{~K}$ as a function of electron concentration, using $\mathrm{f}=2$.

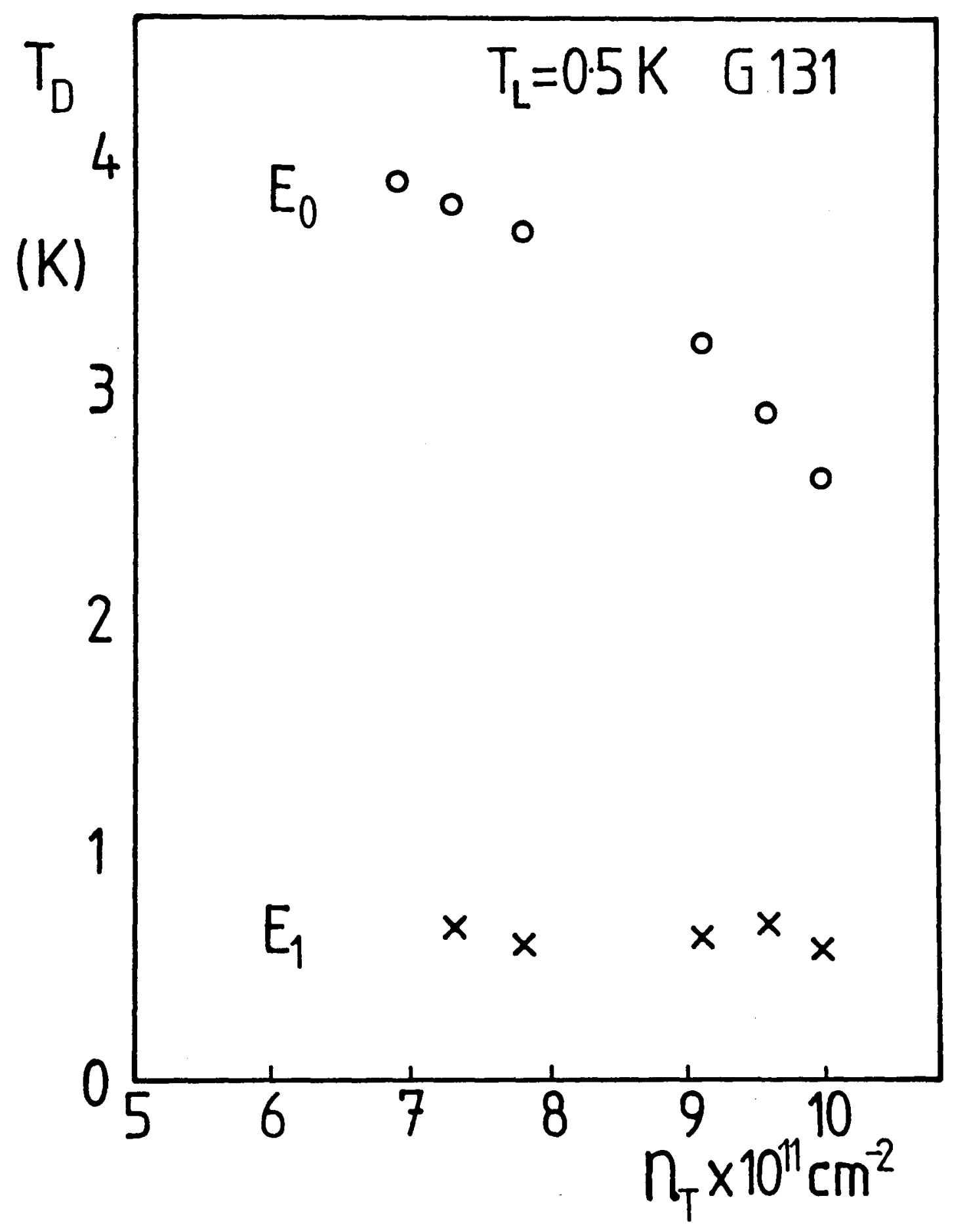

Figure 7.2 Change of Dingle temperature with electron concentration in the two subbands of G131. 


\begin{tabular}{|c|c|c|c|c|c|c|}
\hline Sample & $\underset{\dot{A}}{\text { Spacer }}$ & $\begin{array}{c}\mathrm{n}_{\mathrm{o}} \\
10^{1}\end{array}$ & & $\begin{array}{l}\tau_{\mathrm{t}} \\
\mathrm{ps}\end{array}$ & $\begin{array}{l}{ }^{\tau} s \\
\text { ps }\end{array}$ & $\frac{\tau_{S}}{\tau_{t}}$ \\
\hline G139 & 1600 & $0.92 *$ & $*$ & 36 & 1.3 & 0.036 \\
\hline G137 & $"$ & $0.95 *$ & * & 39 & 1.2 & 0.031 \\
\hline G142 & 800 & 1.2 & & 21 & 0.6 & 0.027 \\
\hline G156 & 800 & $1.7 *$ & $*$ & 40 & 1.6 & 0.040 \\
\hline G148 & 400 & 2.9 & & 50 & 0.8 & 0.016 \\
\hline G 29 & $"$ & $3.4 *$ & $*$ & 65 & 1.1 & 0.016 \\
\hline$\underset{n}{\mathrm{G} 104}$ & 200 & $\begin{array}{l}2.6 \\
5.4 *\end{array}$ & * & $\begin{array}{l}12 \\
35\end{array}$ & $\begin{array}{l}0.4 \\
1.4\end{array}$ & $\begin{array}{l}0.034 \\
0.040\end{array}$ \\
\hline $\begin{array}{l}\text { G215 } \\
\text { ( l ower } \\
\text { " } \\
\text { (upper }\end{array}$ & 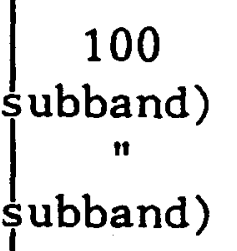 & $\begin{array}{l}4.8 \\
6.9 \\
7.6 * \\
0.55 *\end{array}$ & * & $\begin{array}{l}18 \\
24 \\
26 \\
6.6\end{array}$ & $\begin{array}{l}0.4 \\
0.8 \\
1.2 \\
2.1\end{array}$ & $\begin{array}{l}0.024 \\
0.032 \\
0.046 \\
0.32\end{array}$ \\
\hline $\begin{array}{c}\text { G131 } \\
\text { (1 ower } \\
\text { " } \\
\text { (upper) }\end{array}$ & $\begin{array}{c}17 \\
\text { ubband) } \\
" \\
"\end{array}$ & $\begin{array}{l}6.6 \\
8.5 \\
9.2 \quad * \\
0.8 \quad *\end{array}$ & * & $\begin{array}{l}3.3 \\
4.2 \\
4.5 \\
4.5\end{array}$ & $\begin{array}{l}0.31 \\
0.38 \\
0.46 \\
2.1\end{array}$ & $\begin{array}{l}0.094 \\
0.090 \\
0.090 \\
0.47\end{array}$ \\
\hline
\end{tabular}

Table 7.1

* indicates the sample was fully photoexcited.

From these data the following trends emerge:

(i) $\quad \tau_{s} / \tau_{t} \ll 1$ for all samples studied

(ii) this ratio is similar for all samples except G148, G29 and G131

(iii) $\tau_{S}$ is much larger after illumination than in the dark and varies little from sample to sample

(iv) when two subbands are populated $\tau_{\mathrm{S}}$ is much longer in the upper subband.

The first point shows that the predominant scattering is small angled, as would be expected in modulation doped structures where the mobility is dominated by remote impurity scattering. In their calculations Walukiewicz et al. (1984) found this to be true below $\sim 10 \mathrm{~K}$ and that at higher temperatures acoustic phonon scattering was more important.

The approximate constancy of $\tau_{s} \tau_{t}$ shows the scattering probability, $P_{k, k^{*}}$ of Eqns. 7.2 \& 7.3, has the same angular dependence in all the samples, indicating that 
Figure 7.3 $\tau_{t}$ and $\tau_{s}$ shown as a function of spacer layer thichness. Solid circles are measurements in the dark; open circles after photoexcitation. Lines are calculations of Das Sarma \& Stern. Additional experimental data pointa are from Paalanen et al. $-\mathrm{x}$ and Fang et al. $-\diamond, \diamond$.

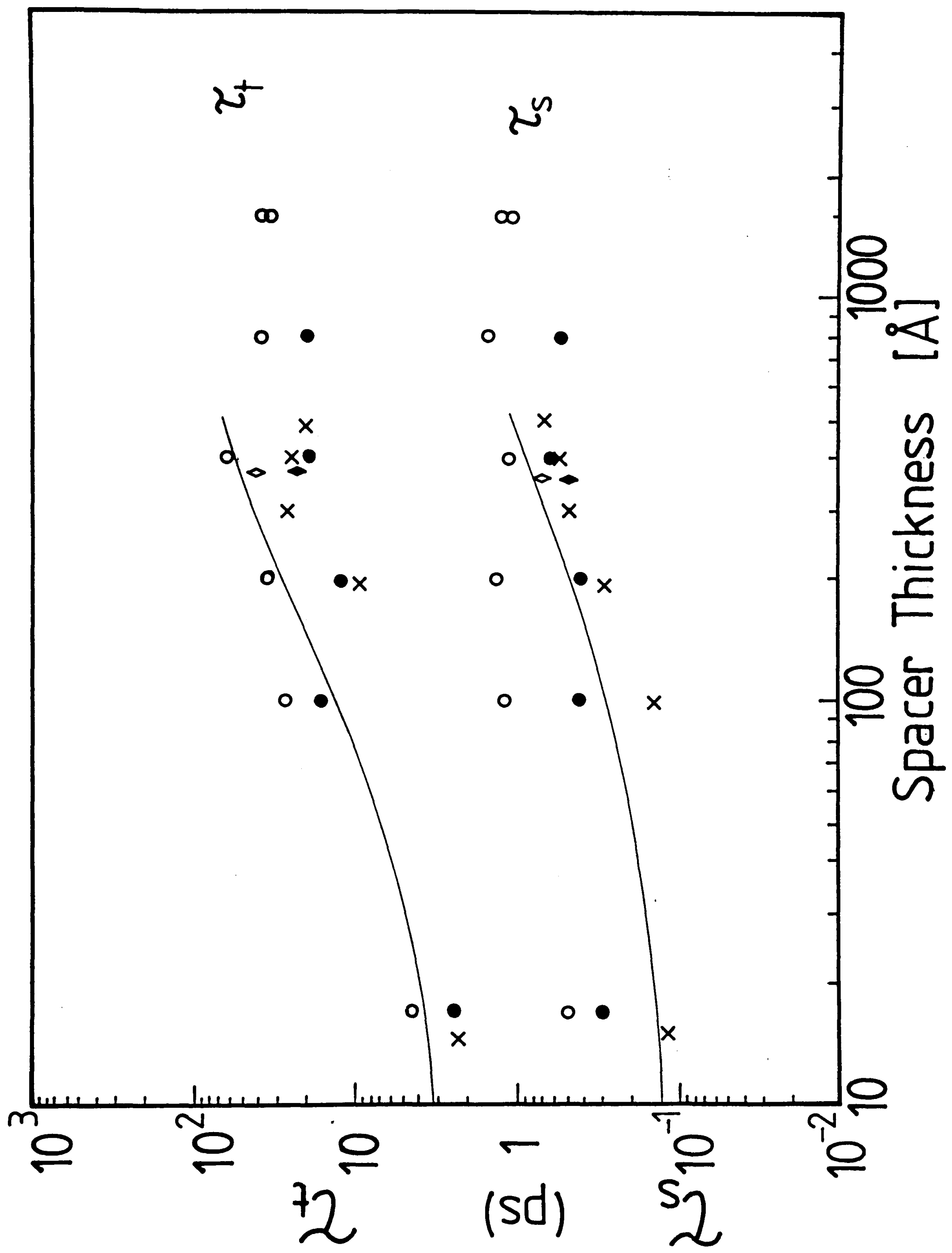


the dominant scattering mechanism is the same. That the value of this ratio is lower in G148 and G29 is a consequence of a more rapid change in $\tau_{t}$, as $\tau_{s}$ has a similar value to other samples as shown in Fig. 7.3. These samples both having a $400 \AA \dot{A}$ spacer layer which seems to produce the maximum transport mobility: thinner spacers mean the ionized impurities in the GaAlAs are nearer the electrons, but as the width increases there are less electrons in the 2DEG so their wavevectors are smaller and the long range scattering has less effect and there will also be less screening of the background impurities in the GaAs. Walukiewicz et al. also calculated the effect on the mobility of changing the spacer width, finding scattering from background impurities becomes more important than from remote impurities with wider spacer layers. Using a background impurity concentration of $\mathrm{N}_{\mathrm{i}}=1 \times 10^{15} \mathrm{~cm}^{-3}$ the maximum in $\mu$ occured with a spacer layer of $\sim 160 \dot{A}$. Our transport mobilities peak for $400 \dot{A}$ spacers indicative of a smaller background impurity density in these samples which can be estimated, by assuming the scattering rate scales with $N_{i}$, as $\sim 2 \times 10^{14} \mathrm{~cm}^{-3}$, which is in the range expected by Foxon \& Harris (1986).

In G131 there is a much thinner spacer layer resulting in a lower transport mobility, but here $\tau_{\mathrm{s}}$ is also smaller.

The changes of $\tau_{s}$ on increasing $n_{s}$ will depend on exactly how the carriers are produced as discussed in Section 1.2.4. For varying $\mathrm{n}_{\mathrm{s}}$ by increasing the GaAlAs doping level calculations have been made by Das Sarma \& Stern (1985) (in fact they change $\mathrm{k}_{\mathrm{F}} / \mathrm{q}_{\mathrm{TF}}$, where $\mathrm{q}_{\mathrm{TF}}$ is the Thomas-Fermi screening constant, which is equivalent). This produces more remote scattering centres and so causes $\tau_{s} \tau_{t}$ to decrease as the carrier density increases in a given sample. However, increasing the number of impurities affects the transport mobility adversely and so is not ideal in a practical situation.

Fig. 7.3 depicts the calculations of Das Sarma \& Stern together with experimental results of Paalanen et al. (1983), Fang et al. (1988) and those given in Table 7.1 as a function of spacer layer thickness. Although previous results and the calculations predict a decrease in both times as the spacer is narrowed, our results show $\tau_{s}$ to be constant in all samples after the same amount of illumination, with the exception of G131. It 
also appears that photoexcitation brings a considerable enhancement in the lifetimes.

Using persistent photoconductivity, carriers are produced by either freeing trapped charges from deep levels (typically DX centres Lang 1986, Higgins et al. 1987) or exciting electron-hole pairs in the GaAs. Mani \& Anderson (1988) have discussed the effect of DX centre ionisation on $\tau_{s} \tau_{t}$ and suggest that the excitation reduces the charge state of the traps, reducing the amount of small angle scattering and so increasing $\tau_{\mathbf{s}} \tau_{\mathrm{t}}$ However the behaviour of DX centres is not very well understood (Lang \& Logan 1977, Hjalmarson \& Drummond 1986, Maude et al. 1987) and it is possible that the charge state may increase. These measurements show $\tau_{s}$ more than doubles on photoexcitation, which supports the idea of less scattering after illumination. This may merely be evidence of the change in $\mathrm{k}_{\mathrm{F}}$ as the carrier concentration is increased, but would otherwise suggest the trap states are neutralised on giving up their electrons. In G131 Harris et al. (1987) claimed phototexcitation with a red LED was mostly by pair production in the GaAs, giving more screening in the 2DEG without the additional scattering centres.

The fourth point is that $\tau_{s}$ is always longer in the upper subband. Electrons in the upper subband will be further from the interface and so affected to a lesser extent by the ionized impurities, resulting in a longer $\tau_{s^{\prime}}$. They will also have a much smaller Fermi wavevector than those in the lower subband. So for a given scattering event, producing a wavevector change $\Delta \mathbf{k}$, the scattering angle will be larger making $\tau_{s} \tau_{t}$ closer to unity as observed in all cases and calculated by Das Sarma \& Stern (1985) as a function of $k_{\mathrm{F}} / \mathrm{q}_{\mathrm{TF}}$. Relative mobilities of electrons in the two subbands will be discussed further below.

\subsection{UPPER SUBBAND TRANSPORT MOBILITY}

The transport mobilities of each subband can be calculated in two ways:

(i) The Hall data, $\mathrm{n}_{\mathrm{H}}$ and $\mu_{\mathrm{H}}$, can be fitted (Stormer et al. 1982) - for $\mathrm{n}_{0} \gg \mathrm{n}_{1}$, $\mathrm{n}_{\mathrm{h}} \sim \mathrm{n}_{0}$ and:

$$
\mu_{0} \approx \mu_{\mathrm{H}} \quad \text { and } \quad \mu_{1} \approx \mu_{\mathrm{H}}-\left[\frac{\mathrm{n}_{0}+\mathrm{n}_{1}-\mathrm{n}_{\mathrm{H}}}{\mathrm{n}_{1}}\right]^{\frac{1}{2}}
$$


To achieve reliable values of $\mu_{1}$ when $n_{1}$ is small $\rho_{x y}$ must be measured to better than $0.1 \%$, so the errors tend to be rather large. Störmer et al. (1982) found $\mu_{1} \approx 2.8$ and $\mu_{0}=4.0 \mathrm{~V}^{-1} \mathrm{~s}^{-1} \mathrm{~m}^{2}$ in a sample with $\mathrm{n}_{\mathrm{s}}=9.3 \times 10^{11} \mathrm{~cm}^{-2}$.

(ii) Better is to fit the low field increase in $\rho_{\mathrm{xx}}$ to the classical formula for two independent carrier types (Section 2.2.3, Smith 1978):-

$$
\frac{\Delta \rho_{\mathrm{xx}}}{\rho_{\mathrm{xx}}}=\frac{\sigma_{0} \sigma_{1}\left(\mu_{0}-\mu_{1}\right)^{2 \mathrm{~B}^{2}}}{\sigma_{\mathrm{T}}{ }^{2}+\left[\mu_{0} \mu_{1}\left(\mathrm{n}_{0}+\mathrm{n}_{1}\right) \mathrm{eB}\right]^{2}}
$$

where $\sigma_{\mathrm{i}}=\mathrm{n}_{\mathrm{i}} \mathrm{e} \mu_{\mathrm{i}}$ and $\sigma_{\mathrm{T}}=\sigma_{0}+\sigma_{1}$. If $\mu_{0}=\mu_{1}$, or only one subband is populated, $\Delta \rho=0$, but there will be a positive magnetoresistance for both $\mu_{0} \geqslant \mu_{1}$ which will saturate in high fields at:

$$
\frac{\Delta \rho_{\max }}{\rho}=\frac{\mathrm{n}_{1}}{\mathrm{n}_{0}} \frac{\left(\mu_{0}-\mu_{1}\right)^{2}}{\mu_{0} \mu_{1}}
$$

However, if $\mu_{1}>\mu_{0}$ this saturation value will be reached at much lower fields, typically $<0.02 \mathrm{~T}$ as shown in Fig. 7.4 by the broken curve, compared with $0.2 \mathrm{~T}$ when the

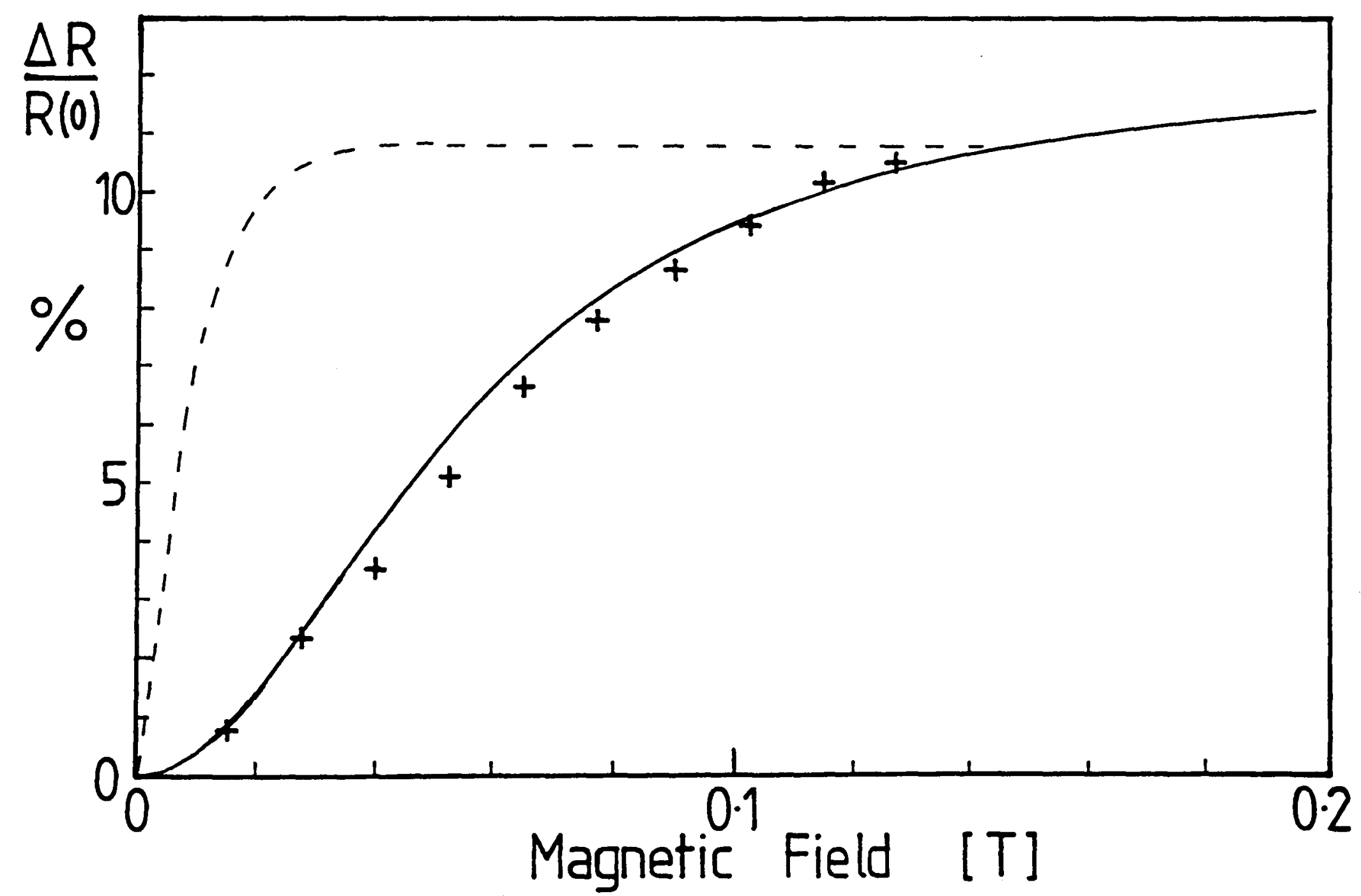

Figure 7.4 Change in resistance at low magnetic fields due to upper subband in G215. 
lower subband has the greater mobility - solid curve in Fig. 7.4, which shows far better agreement with the experimental data from G215.

For the carrier concentrations pertaining to Fig. 7.4 the respective mobilities of the $E_{0}$ and $E_{1}$ subbands are found to be 65 and $17 \mathrm{~V}^{-1} \mathrm{~s}^{-1} \mathrm{~m}^{2}$, in agreement with van Houten et al. (1988) and Smith et al. (1988) using similar quasi-accumulation type samples. The mobility of each subband exhibits a similar carrier concentration dependence, as shown by the systematic results of Fig. 7.5. In contrast G131 shows no positive magnetoresistance and so one must assume $\mu_{1}=\mu_{0} \approx 13 \mathrm{~V}^{-1} \mathrm{~s}^{-1} \mathrm{~m}^{2}$. Further in a lower quality sample Smith \& Fang (1988) found $\mu_{1}=16.5$ and $\mu_{0}=6.6 \mathrm{~V}^{-1} \mathrm{~s}^{-1} \mathrm{~m}^{2}$. Thus it appears that while the mobility of the lower subband varies over an order of magnitude there is relatively little change in the upper subband mobility. However, as shown above, all two subband samples studied show $\tau_{s}$ to be much greater for electrons in the upper subband (but still $<\tau_{\mathrm{t}}$ ).

There is always a much greater density of electrons in the lower subband whose distribution is peaked closer to the interface than for the upper subband. This may give a very effective screening of the upper subband electrons from scattering centres in the GaAlAs and associated with the interface. The scattering of $E_{1}$ electrons would then be determined more by the purity of the GaAs channel, since their wavefunctions extend further from the interface where there would be less screening provided by the lower subband electrons. It is reasonable to assume that there will be many less scattering centres in the GaAs so $\tau_{s}$ will always be greater in the upper subband. By the same arguement changes in spacer layer thickness and GaAlAs doping will have a much larger effect on $\tau_{t 0}$ than $\tau_{\mathrm{t} 1}$. Although this is sufficient to account for the constancy of $\tau_{\mathrm{t} 1}$, it does not necessarily explain how the electrons of the upper subband can have a larger mobility when their Fermi wavevector is so much smaller than those of the lower subband (taking typical values $\mathrm{k}_{\mathrm{F} 1} \sim \frac{1}{4} \mathrm{k}_{\mathrm{F} 0}$ ). The aditional screening of the $\mathrm{E}_{0}$ electrons will help, but exact values of $\tau_{\mathrm{tl}} / \tau_{\mathrm{t} 0}$ will depend on the detailed distribution of scattering centres. In samples such as $G 215$, which has an unintentionally $\mathrm{n}^{-}$doped buffer and as such is accumulation layer like, the electrons of the upper subband will be spread out to 
a greater extent over these impurities and not be as well screened as those in the lower subband, which results in $\mu_{1}$ being less than $\mu_{0}$ (the impurities are not remote so $\tau_{t 1}$ is much closer in value to $\left.\tau_{s 1}\right)$.

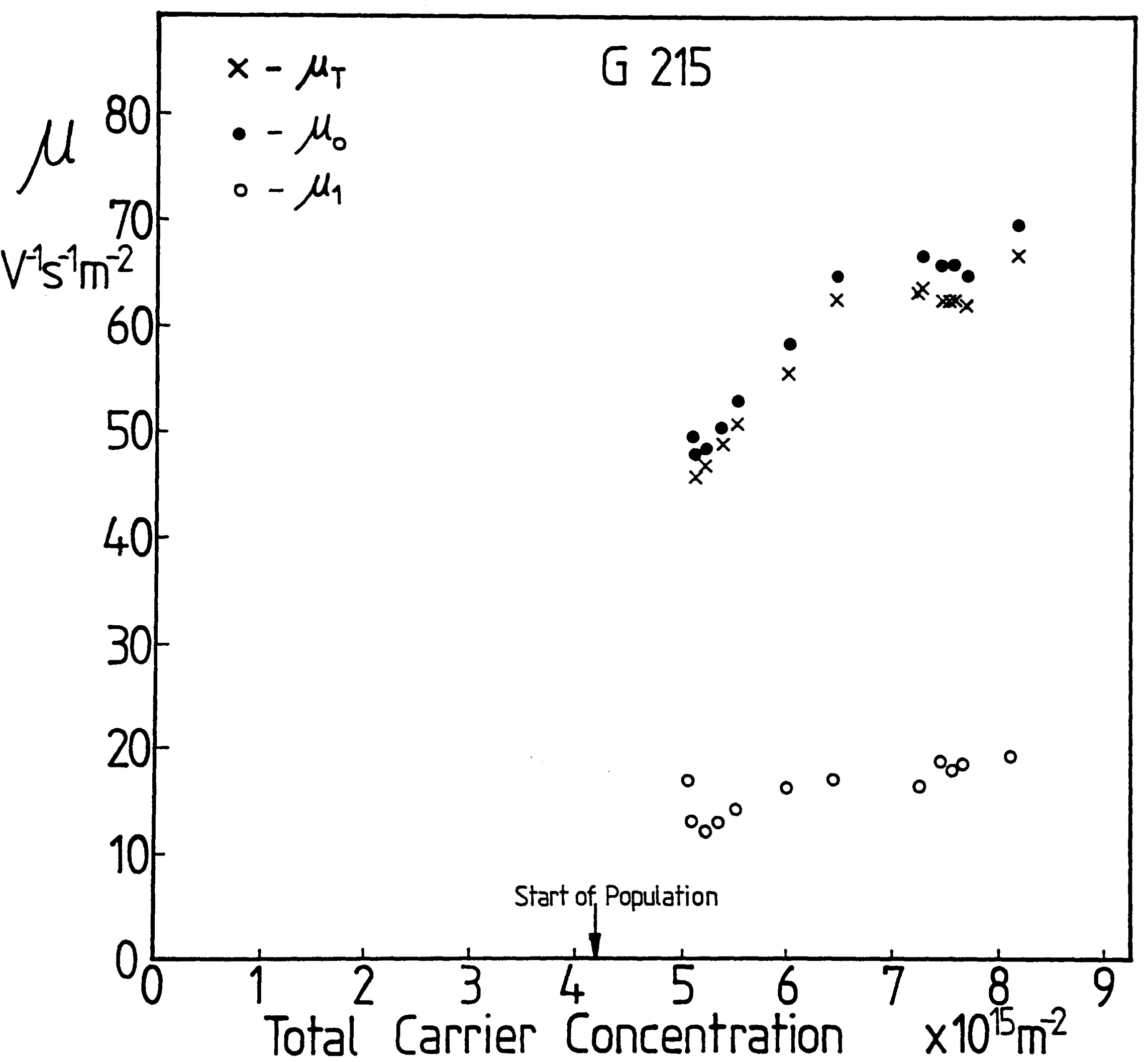

Figure 7.5 Mobility of upper and lower subbands in G215 as the carrier concentration is changed by photoexcitation with a red LED. 


\subsection{OTHER SOURCES OF LOW FIELD MAGNETORESISTANCE}

At low temperatures and weak magnetic fields there are other phenomena which affect the magnetoresistance (Paalanen et al. 1983 and refs. therin). One of these, the weak localisation correction, results from the properties of a single electron in a random potential, but only for $\omega_{c} \tau_{t} \ll 1$ and so would not influence our results. The other is due to the enhanced Coulomb interaction between diffusively moving electrons. This interaction correction gives a parabolic negative magnetoresistance at low fields before Shubnikov-deHaas oscillations appear.

$$
\frac{\Delta \rho}{\rho^{2}}=-\frac{\mathrm{e}^{2}}{2 \pi^{2} \hbar}\left[1-\left(\omega_{\mathrm{c}^{\top} t}\right)^{2}\right](1-\mathrm{F}) \ln \left[\frac{\mathrm{kT} \tau}{\hbar}\right]
$$

where $(1-F)$ is the interaction coefficient. Paalanen et al. (1983) used this to find a value of $\tau$ in a single subband sample in agreement with $\tau_{s}$ deduced from Shubnikov-deHaas oscillations at higher fields.

However, if this effect were also present in a two-subband sample it would affect values of $\mu_{0}$ and $\mu_{1}$ deduced from Eqn. 7.8, unless a suitable correction was applied which is not known to have been made in any of the published work and involves the non-trivial calculation of $\mathrm{F}$.

From repeated measurements on these GaAs-GaAlAs heterojunctions the amount of negative magnetoresistance seems to increase with thermal cycling and at the same time the zero field mobility deteriorates. These two observations suggest that $\tau_{t}$ should be measured at a magnetic field above the region of negative magnetoresistance - this would give a more constant mobility over the life of the sample. The reason for the decreased mobility as the sample ages is probably due to degradation of the surface, giving a less uniform charge distribution in the 2DEG. Other possible changes could include diffusive processes around the contact pads as a result of thermal cycling. The sample properties in high field should be less adversely affected as the sample is probably still homogeneous on the scale of the cyclotron radius. 
Figure 7.6 Magnetoresistance of G215 rotated in a constant field of 5.75T, showing an unexplained dip near the parallel field configuration and Shubnikov-deHaas oscillations on further rotation.

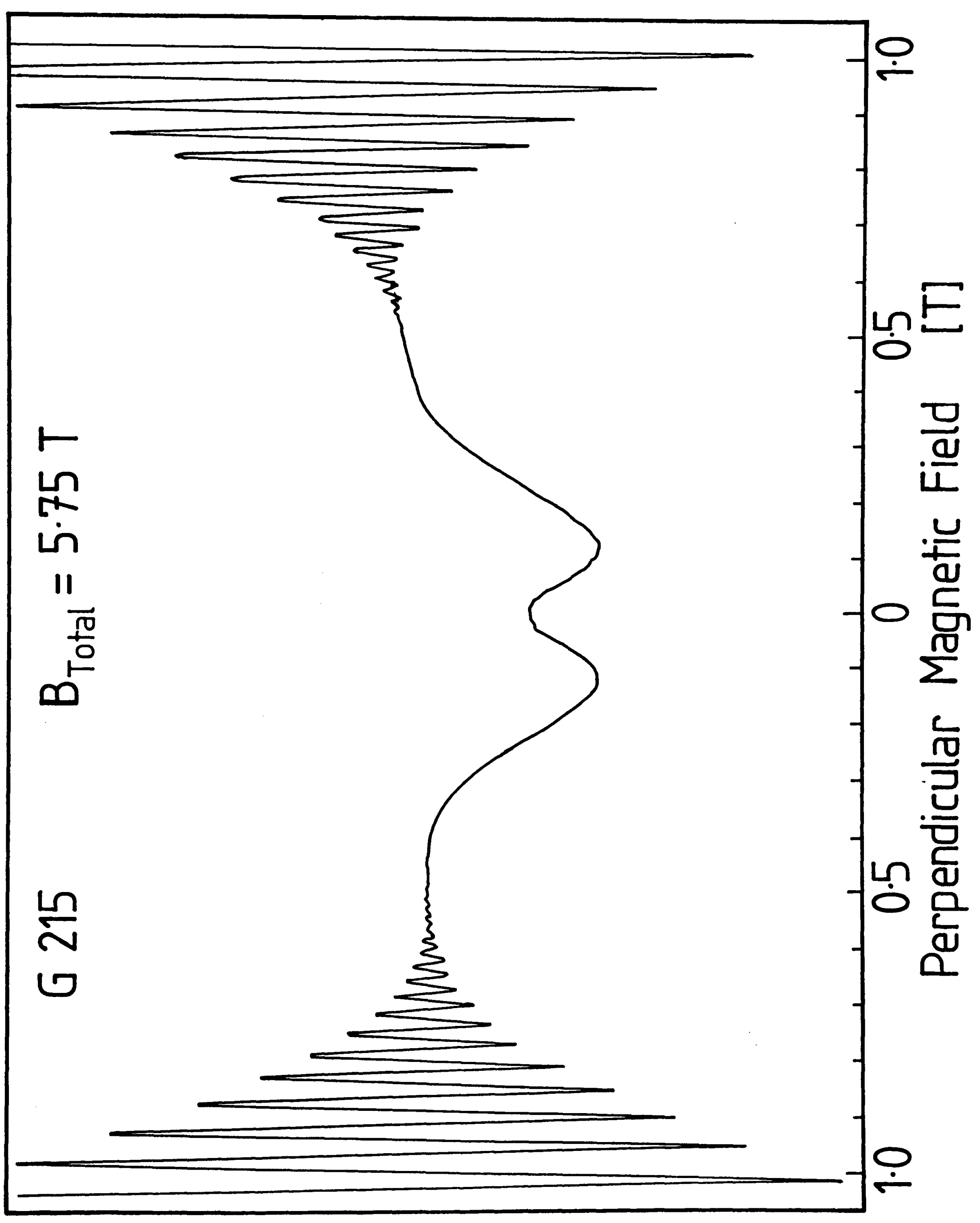




\section{Wierd Structure at High Tilt Angles}

Other evidence for problems with Eqn. 7.8 come from resistivity measurements in large parallel fields, where if a small perpendicular field is applied by tilting the sample, Shubnikov-deHaas oscillations are again seen. However, around $B_{\perp}=0$ (ie. $\theta=90^{\circ}$ where $\theta$ is the angle between the magnetic field and the normal to the 2DEG) there is a distinct dip in the resistivity as shown in Fig. 7.6. This was first observed in GaAs-GaAlAs by Englert et al. (1983) and can be seen in other materials eg. GaInAs-InP and InSb grain boundaries (Herrmann et al. 1985). At the parallel fields used all upper subbands will be depopulated so it is difficult to reconcile this result with that seen in small perpendicular fields with $B_{\perp}=0$ described by Eqn. 7.8.

Consequently this effect was studied in G215 at different values of $B_{\text {Total }}$ and was found to much stronger at higher fields and almost to dissappear at magnetic fields insufficient to totally depopulate the upper subband. Figure 7.7 shows the parallel field magnetoresistance with the effects of rotating the sample a few degrees away from this configuration at five values of total field superimposed. Herrmann et al. (1985) only show this structure at a parallel field of $15 \mathrm{~T}$ and not below 5T. It may be that the small amount of perpendicular field mixes the subbands and so reactivates the intersubband scattering. However, the full details require further study, both experimental and theoretical. Also, at higher parallel fields additional structure is seen around $\theta=90^{\circ}$, for which there is no satisfactory explaination. 
Figure 7.7 Parallel field magnetoresistance of G215 (thick line), with superimposed at various magnetic fields, the effects of a few degrees rotation at constant total field.

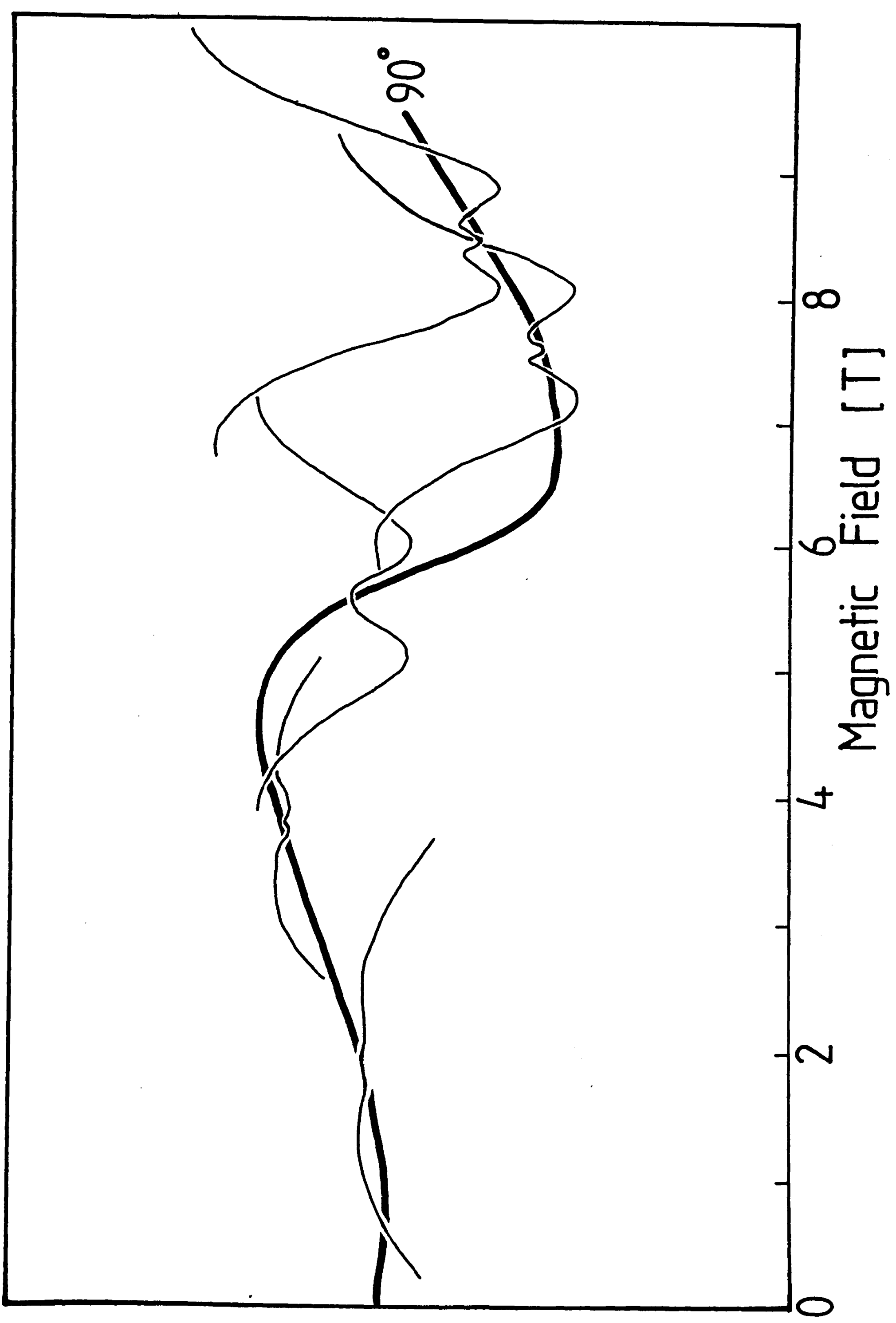




\subsection{SUMMARY}

The essential difference between $\tau_{t}$ - measured from the transport mobility - and the quantum lifetime, $\tau_{s}$ - measured from the damping in magnetic field of Shubnikov-deHaas oscillations - have been discussed. In all cases $\tau_{\mathfrak{t}}$ is much greater than $\tau_{s}$ showing that remote ionised impurity scattering is the dominant scattering mechanism affecting the $2 D E G$ at low temperatures.

Photoexciting more carriers in a given sample increases both times - a much larger change than between samples of different spacer widths, showing a reduction in scattering which may be caused by releasing electrons from negatively charged trap states.

When two subbands are populated $\tau_{s}$ is always much longer for electrons in the upper subband, which is taken as evidence for screening from the donors in the GaAlAs by the much larger lower subband population nearer the interface.

$\tau_{t}$ has been measured for each subband from the positive magnetoresistance below $0.2 \mathrm{~T}$ and is found to be greater for the upper subband in low mobility samples, and for the lower subband in high mobility samples. However, this sample variation mainly comes from the lower subband mobility, with a roughly constant value in the upper subband.

Finally, other sources of low field changes to the magnetoresistance were considered, including some very stiking results of the effects of small perpendicular fields in the presence of a large magnetic field parallel to the 2DEG plane. 


\section{CHAPTER 8}

\section{CYCLOTRON PHONON EMISSION AND ELECTRON ENERGY LOSS RATES IN GaAs-GaAlAs HETEROJUNCTIONS}

$\begin{array}{lll}8.1 & \text { Introduction } & 218 \\ 8.2 & \text { Measurement of Electron Temperature } & 220 \\ 8.3 & \text { Enhancement of Loss Rate in Small Magnetic Fields } & 226 \\ 8.4 & \text { Cyclotron Phonon Emission } & 229 \\ 8.5 & \text { Intra-Landau Level Energy Loss } & 231 \\ 8.6 & \text { Summary } & 233\end{array}$




\section{$\underline{8.1 \quad \text { INTRODUCTION }}$}

Electron energy loss has been studied in 2-D systems by a variety of both optical and electrical techniques in the temperature range $10 \mathrm{mK}-400 \mathrm{~K}$, which, in GaAs-GaAlAs heterostructures, show loss rates per electron ranging over 18 orders of magnitude (Fig. 8.1). All of these, from time-resolved studies of carriers cooling after optical excitation (Shah 1986, Hollering et al. 1988) through the luminescence (Yang et al. 1985, Shah et al. 1985) and FIR emission (Höpfel et al. 1985) of electrically heated systems to reaching the lowest temperatures for study of the Fractional Quantum Hall Effect (Gammel et al. 1988), address the problem of how hot electrons lose energy to the crystal lattice. In most cases the energy loss mechanism is dominated by either acoustic or optic phonon emission, although some recent calculations predict important cotributions from each mechanism well outside their expected temperature regimes (Hollering et al. 1988, Das Sarma 1988). Many of these studies have been made in magnetic fields between $\sim 2$ and $20 \mathrm{~T}$, but it is only at the highest fields (Hollering et al. 1988), and with electron temperatures $>50 \mathrm{~K}$, that any variation in the electron energy loss rates has been observed.

In this study, electron heating and energy loss in the low temperature region $(0.5 \mathrm{~K}-10 \mathrm{~K})$ was investigated, together with the influence of relatively weak magnetic fields. The chosen technique was to use the amplitude of the Shubnikov-deHaas. oscillations at various magnetic fields to deduce an electron temperature $\left(T_{e}\right)$ as a function of electric field. This method has been used in a variety of systems, eg. Kahlert \& Bauer (1971) in bulk GaSb; Neugebauer \& Landwehr (1980) and Dolgopolov et al. (1985) in Si inversion layers; Barlow et al. (1988) in GaInAs-InP heterostructures; and in GaAs-GaAlAs heterojunctions these results will be compared with those of Sakaki et al. (1984), Hirakawa \& Sakaki (1986) and Manion et al. (1987).

In contast to previous measurements, the electron energy loss rate is found to be strongly dependent on both the magnetic and electric field values (and hence the electron temperature). This is interpreted as a change in the relative importance of 
Figure 8.1 Eighteen orders of magnitude change of energy loss rate per electron as a function of temperature. The results and calculations are from this work and those indicated on the figure.

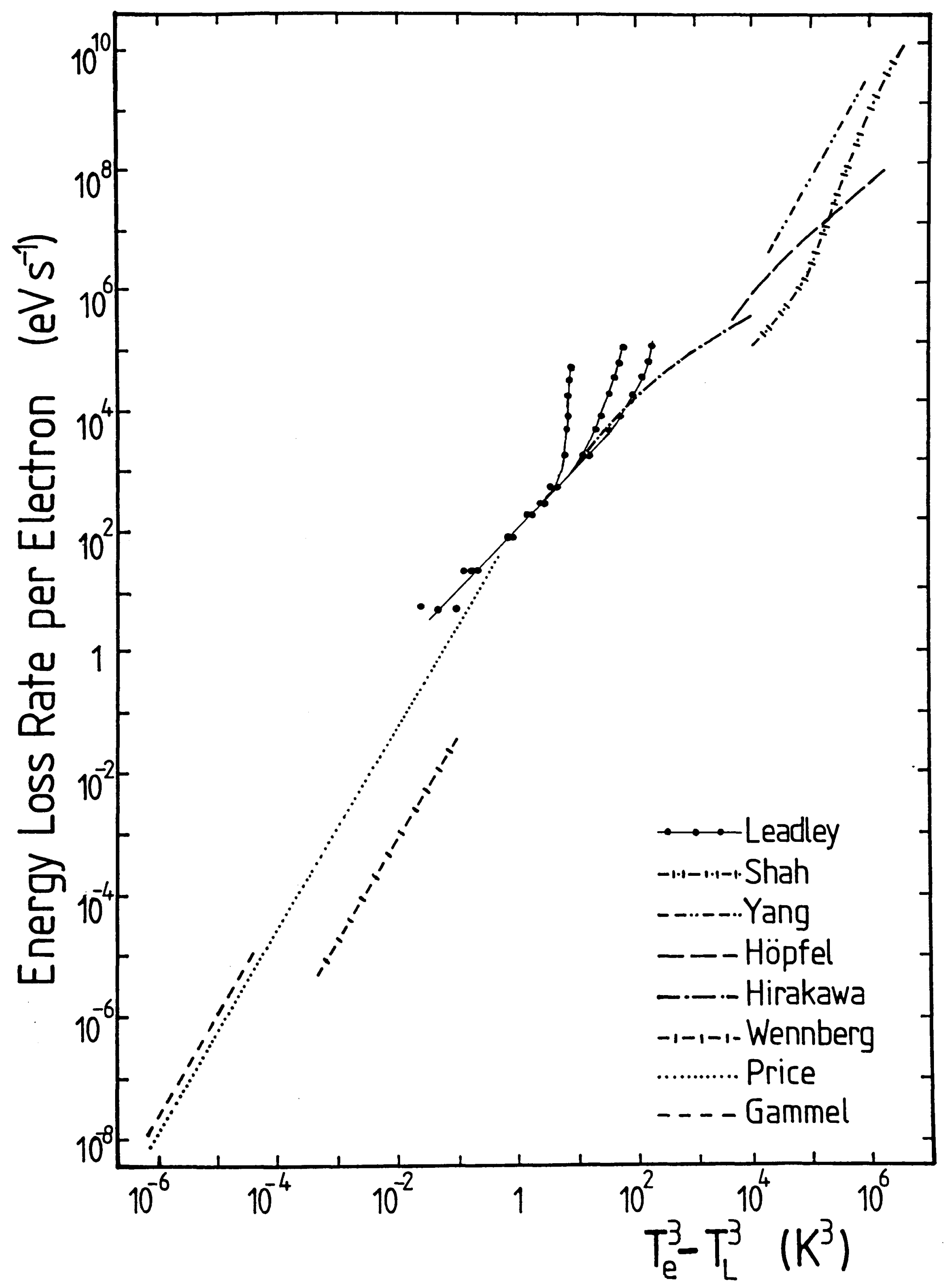


intra- and inter-Landau level phonon emission, and the dominance at high electron temperatures of the 'cyclotron phonon' emission process, first predicted by Slater \& Tremblay (1984).

\subsection{MEASUREMENT OF ELECTRON TEMPERATURE}

The samples chosen for study included structures with both single and double subband occupancy and were discussed in Chapter 2. All were photoexcited by a red LED to give the maximum possible mobilities, which are considerably higher than those used by Manion et al. (1987) and Hirakawa \& Sakaki (1986). This enables Shubnikov-deHaas oscillations to be discerned at much lower magnetic fields where the cyclotron energy may be less than $\mathrm{kT}_{\mathrm{e}}$. The relevent sample parameters as used in these experiments are shown in Table 8.1.

\begin{tabular}{|c|c|c|c|c|}
\hline Sample & $\begin{array}{c}\text { Spacer Layer } \\
\dot{\mathrm{A}}\end{array}$ & $\begin{array}{c}\mathrm{n}_{0} \\
10^{1} \mathrm{~cm}^{-2}\end{array}$ & $\begin{array}{c}\mathrm{n}_{1} \\
10^{1} \mathrm{~cm}^{-2}\end{array}$ & $\begin{array}{c}\mu_{4 \mathrm{~K}} \\
\mathrm{~m}^{2} \mathrm{~V}^{-1} \mathrm{~s}^{-1}\end{array}$ \\
\hline G156 & 800 & 1.7 & - & 103 \\
G104 & 200 & 5.4 & $<0.1$ & 151 \\
G215 & 100 & 7.2 & 0.45 & 75 \\
\hline
\end{tabular}

Table 8.1

A typical experimental recording is shown in Fig. 8.2 for G104 at low electric field. Notice that in the region where oscillations are seen $\mu \mathrm{B} \geqslant 1$, with $\mu$ being the d.c. mobility, so we are in the 'high-field' limit even below $0.1 \mathrm{~T}$.

As the electric field along the bar was increased the amplitude of the oscillations decreased, as shown in Fig. 8.3 for G156, which enabled an effective electron temperature to be deduced from

$$
\frac{\Delta \rho}{\rho} \propto \frac{\chi}{\sinh \chi} \exp \left(-\frac{\pi}{\omega_{c^{\top} s}}\right)
$$

where $\chi=\frac{2 \pi^{2} k T}{\hbar \omega_{c}}$

The discussion of Section 2.2 concerning the precise form of this equation and the exact value of $\tau$ will not affect the electron temperatures deduced, as only amplitude 


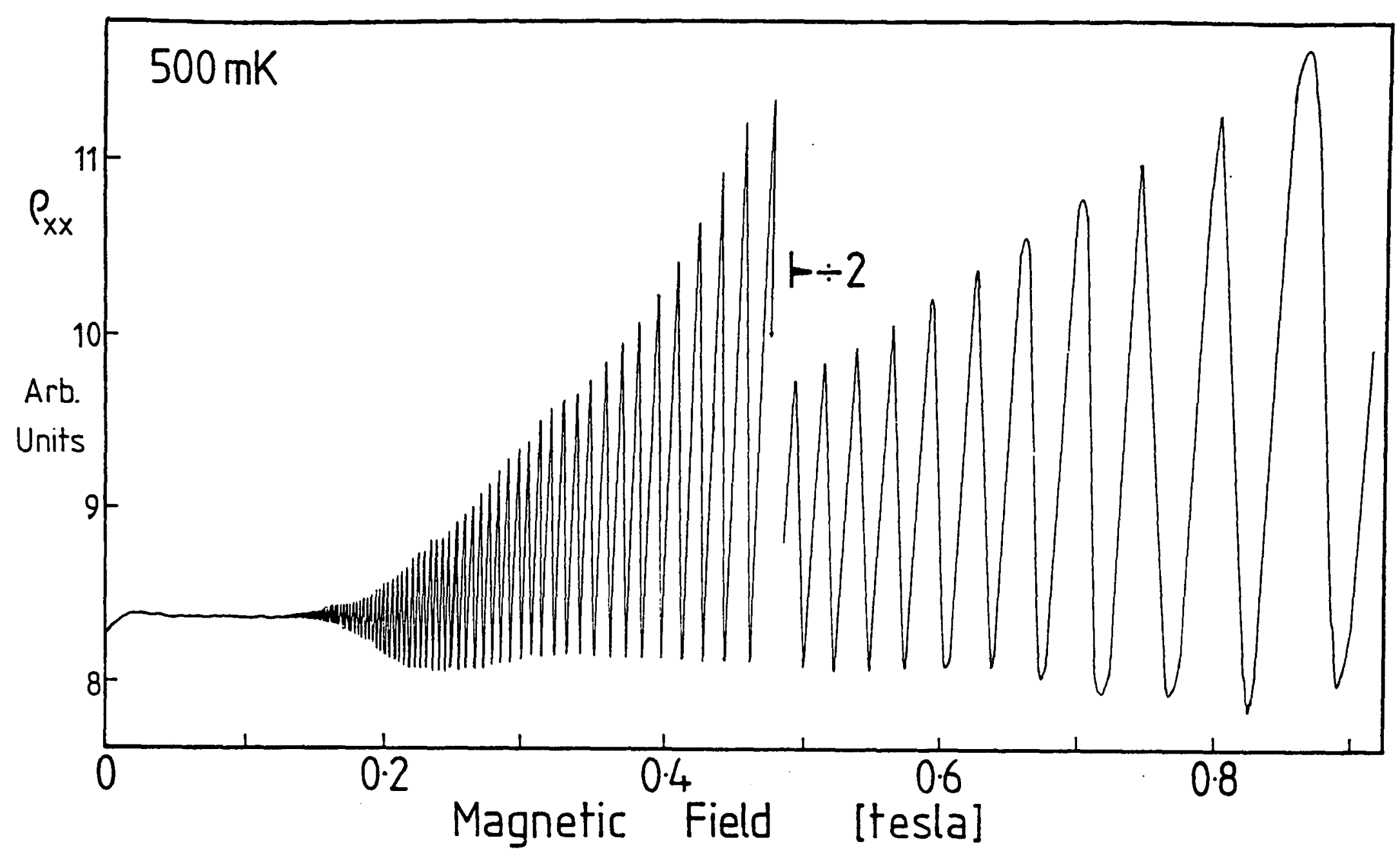

Figure 8.2 Typical Shubnikov-deHaas recording at low magnetic field for G104.

ratios at fixed magnetic field are used. However, at $500 \mathrm{mK}$ the oscillations become large fractions of the resistivity for magnetic fields $>0.6 \mathrm{~T}$. In previous studies (Hirakawa \& Sakaki 1986, Kahlert \& Bauer 1971, Neugebauer \& Landwehr 1980) the Dingle Temperature (ie. $\tau_{\mathrm{s}}$ ) has always been assumed not to vary with electric field and this has been experimentally verified by Mani \& Anderson (1988). Although there is a possibility that it may vary with magnetic field, this would not affect our measurements of ratios at fixed B. However, these considerations require the validity of Eqn. 8.1 to be checked. This was done by measuring the temperature dependence of the oscillations directly for $0.5<\mathrm{T}<4.2 \mathrm{~K}$ at electric fields small enough for the carriers to be in equilibrium with the lattice. The electron temperatures thus deduced were in agreement with the bath temperature, measured from the helium vapour pressure, to within $\pm 10 \%$ with a random variation over magnetic field of $<5 \%$, which must be regarded as the limit of experimental error. Manion et al. (1987) were sceptical of the validity of Eqn. 8.1 and so deduced their electron temperatures purely by comparison with low electric field measurements at higher lattice 


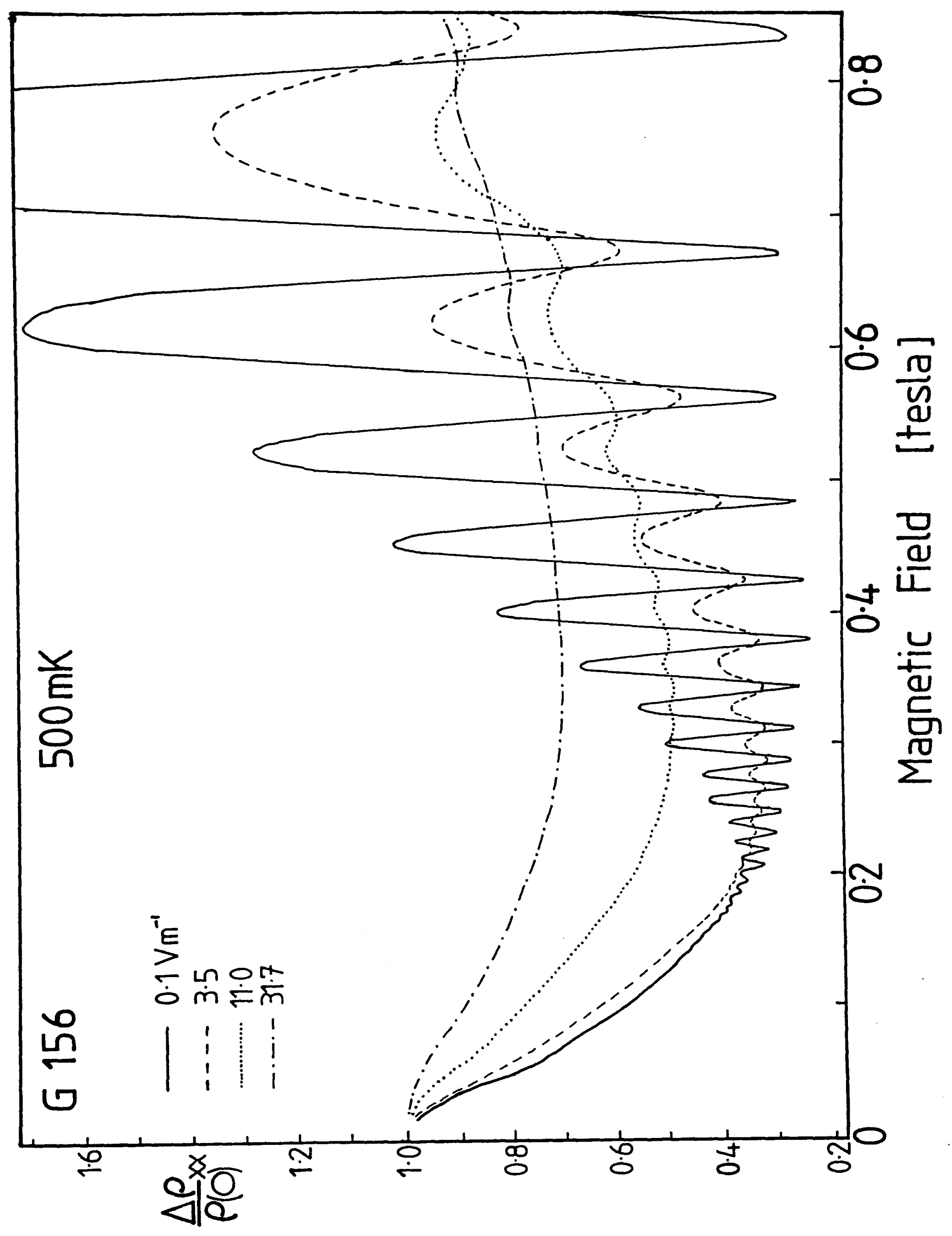

Figure 8.3 Shubnikov-deHaas oscillations from G156 at a lattice temperature of $500 \mathrm{mK}$ showing how the amplitude of the oscillations decreases with increasing electron temperature. 
temperatures. As will be seen shortly their results are in complete agreement with our measurements, provided $\mathrm{kT} / \hbar \omega_{\mathrm{c}}$ is not too large.

The magnetoresistance of Sample G156 was negative for $\mathrm{B}<0.2 \mathrm{~T}$ increasing monotonically thereafter, while the other two samples showed an approximately constant background. For this reason values of $T_{e}$ were deduced by taking the ratio of $\Delta \rho$ to both the zero-field resistivity and the mean value at the magnetic field of interest. There was no noticable difference in the general trends of the results, although the latter method gave more consistent quantitative data. It may be suggested that the electron temperature might fluctuate violently with filling factor, however it should be noted that when the density of states at the Fermi level is a maximum, both the heat capacity and the input power are also maxima giving a roughly constant electron temperature. It was possible to check this from measurements on Sample G215 at low electric fields: no difference was found between the temperatures deduced for electrons in the lowest subband with $E_{F}$ lying in or between upper subband Landau levels.

The majority of measurements were performed using a bath (lattice) temperature, $T_{L}$, of $500 \mathrm{mK}$. Similar results were obtained with $T_{L}=1.5 \mathrm{~K}$, but were less informative due to fewer oscillations being resolved. The electron temperature was deduced for a number of electric fields, using oscillations of differing Landau index to generate a plot of $\mathrm{T}_{e}$ as a function of magnetic field. Typical results are shown in Fig. 8.4 for Sample G215, with similar behaviour being seen for all the samples studied. Some small deviations occurred once the second subband became populated, due to oscillations in the inter-subband scattering rate, found in Section 6.6 (Leadley et al. 1989b). In practice these are hardly visible in plots such as Fig. 8.4, and the electron temperature deduced from both subbands seperately were always found to be in agreement within the limits of experimental error. The same data is plotted as a function of electric field for various magnetic fields in Fig 8.5. This shows clearly how the electron temperature saturates at a magnetic field dependent value of a few kelvin. If the energy relaxation rate were constant an increase 
proportional to $E^{2}$ would be expected, from Eqn. 8.3 below.

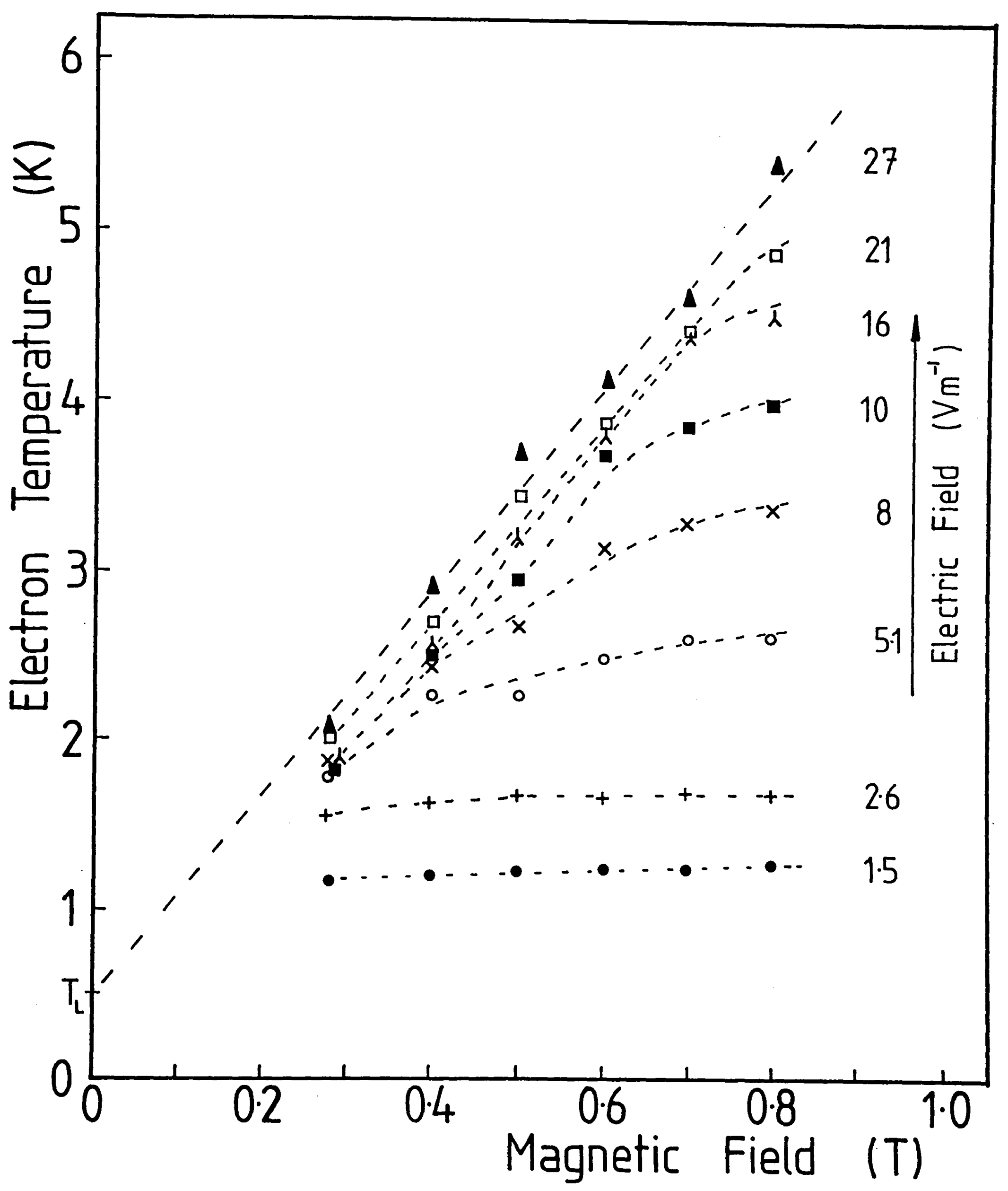

Figure 8.4 The electron temperature deduced from the amplitude of Shubnikov-deHaas oscillations in sample G215, as a function of magnetic field for several electric field strengths indicated on the figure. 
Figure 8.5 The electron temperature deduced from the amplitude of ShubnikovdeHaas oscillations in sample G215, as a function of electric field for several magnetic field values.

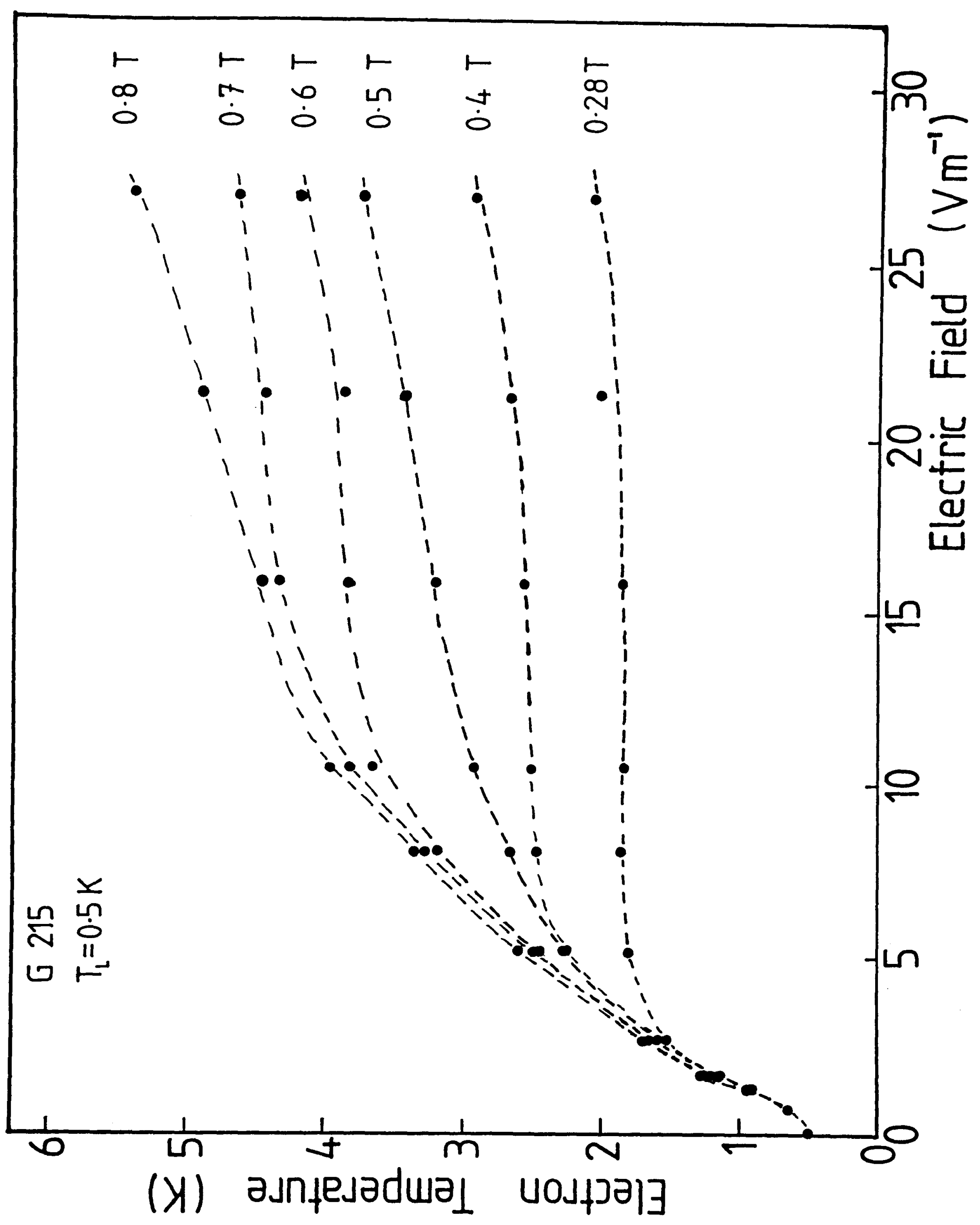




\subsection{ENHANCEMENT OF LOSS RATE IN SMALL MAGNETIC FIELDS}

From Fig. 8.4 it is clear that $\mathrm{T}_{\mathrm{e}}$ is only independent of magnetic field at low electric fields $\left(\underline{E}<3 \mathrm{Vm}^{-1}\right)$. At higher electric fields the electron temperature develops a strong magnetic field dependence, becoming almost linearly proportional to $\underline{B}$, in the large $\underline{E}$, small $\underline{B}$, limit with:-

$$
\lambda \mathrm{kT}_{\mathrm{e}}=\hbar \omega_{\mathrm{c}}
$$

The values of $\lambda$ for each sample are shown in Table 8.2. These results suggest that the magnetic field puts an upper bound on the electron temperature that may be reached, almost regardless of the input power. This behaviour is characteristic of a very strong emission process with a threshold energy, as found for example in L.O. phonon emission.

\begin{tabular}{|c|c|c|c|c|}
\hline Sample & $\begin{array}{c}\mathrm{n}_{\mathrm{s}} \\
10^{1} \mathrm{~cm}^{-2}\end{array}$ & $\lambda$ & $\begin{array}{c}\varphi \\
\mathrm{eVs}^{-1} \mathrm{~K}^{-3}\end{array}$ & $\begin{array}{c}\alpha=\tau^{\tau} \epsilon^{-1} / \mathrm{T} \\
\mathrm{s}^{-1} \mathrm{~K}^{-1}\end{array}$ \\
\hline G156 & 1.7 & 2.3 & 290 & 2.2 \\
G104 & 5.4 & 2.3 & 100 & 2.6 \\
G215 & 7.6 & 3.3 & 110 & 3.6 \\
\hline
\end{tabular}

\section{Table 8.2}

It is possible to investigate the temperature dependence of the energy loss and deduce corresponding electron-phonon scattering rates from these measurements. Assuming the situation is adequetely described by a two-bath model with a well defined electron temperature, due to thermalisation by electron-electron interactions occurring at a much higher rate than the electron-phonon couplings responsible for the energy loss, equilibrium will be reached when the power lost by electron-phonon relaxation, $\dot{Q}$ per electron, equals the input electrical power, $e \mu \mathrm{E}^{2}$, (Kahlert \& Bauer 1971, Wennberg et al. 1986, Hollering et al. 1988). The temperature reached will be determined by the relative sizes of the electron heat 
capacity and energy relaxation rate, $\tau_{\epsilon}^{-1}$,

ie.

$$
\mathrm{d} \dot{\mathrm{Q}}=\tau_{\epsilon}^{-1} \mathrm{C}_{\mathrm{el}} \mathrm{dT} \mathrm{e}
$$

The heat capacity per electron is given by:-

$$
\mathrm{C}_{\mathrm{el}}=\frac{\pi^{2} \mathrm{k}_{\mathrm{B}} \mathrm{T}}{3 \mathrm{~T}_{\mathrm{F}}}=\gamma \mathrm{T}
$$

where $\gamma=1.1 \times 10^{-9} / \mathrm{n}_{\mathrm{s}} J K^{-1}$, with $\mathrm{n}_{\mathrm{s}}$ being the surface electron density. Then assuming the power law $\tau_{\epsilon}^{-1}=\alpha \mathrm{T}^{\mathrm{p}}$ the energy loss rate is:-

$$
\dot{\mathrm{Q}}=\varphi\left(\mathrm{T}_{\left.\mathrm{e}^{\mathrm{p}+2}-\mathrm{T}_{\mathrm{L}}^{\mathrm{p}+2}\right)}\right.
$$

$$
\text { where } \quad \varphi=\frac{\alpha \gamma}{\mathrm{p}+2}
$$

From plots of $\ln \dot{Q}$ against $\ln \mathrm{T}_{e}$ at intermediate temperatures the limiting behaviour is found to be $\dot{\mathrm{Q}} \propto \mathrm{T}^{3}$; in contrast to the low temperature, zero-field behaviour of $\mathrm{T}^{5}$ calculated by Price (1982) and measured from the variation of resistivity with temperature by Wennberg et al. (1986) in a GaAs-GaAlAs heterojunction. (Similar results were found in thin metal films at millikelvin temperatures by Roukes et al. 1985 and Dorozhkin et al. 1986.) Consequently $\dot{Q}$ is plotted as a function of $T_{e}{ }^{3}-T_{L}^{3}$ in Fig. 8.6 for all three samples studied.

It can be seen that there are two regions:

(i) Where $\lambda \mathrm{kT}_{\mathrm{e}}<\hbar \omega_{\mathrm{c}}$ there is a well behaved power law dependence, indicated by the dashed line in Fig. 8.6, with :-

$$
\dot{\mathrm{Q}}=\varphi\left(\mathrm{T}_{e}^{3}-\mathrm{T}_{\mathrm{L}}^{3}\right)
$$

where the constant $\varphi$ is given for each sample in Table 8.2 .

This also seems to be the limiting behaviour at high magnetic fields.

(ii) At higher electron temperatures the loss rate increases extremely rapidly, with the transition to this regime occuring sooner at lower magnetic fields. 


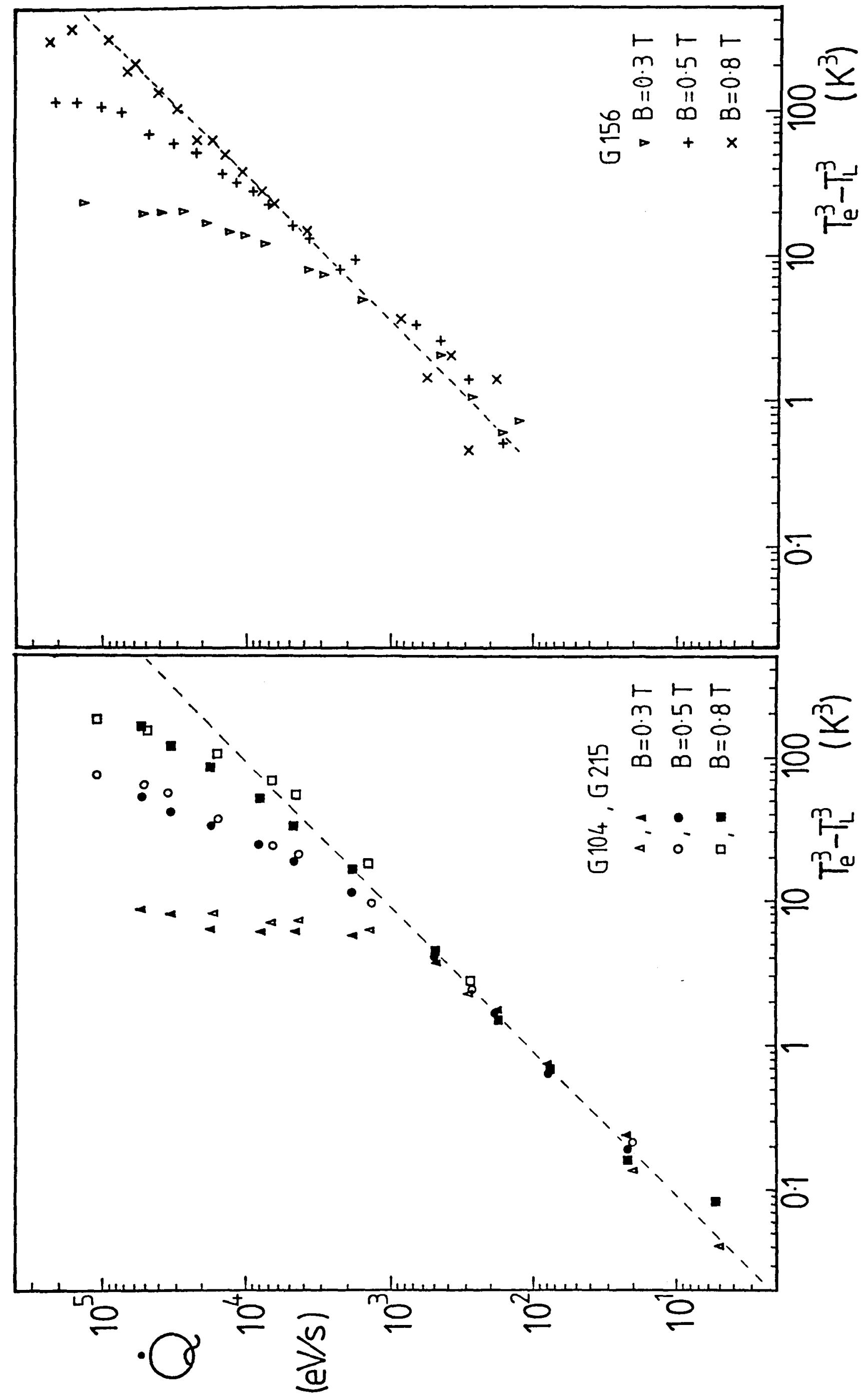

Figure 8.6 Variation of the energy loss rate per electron, $\dot{Q}$, with $\mathrm{T}_{e}{ }^{3}-\mathrm{T}_{\mathrm{L}}{ }^{3}$ for the three samples studied with a lattice temperature of $500 \mathrm{mK}$. The dashed lines are fitted to the low temperature points with unit gradient. Notice the rapid increase away from these lines at low magnetic fields, as cyclotron phonon emission becomes significant. 


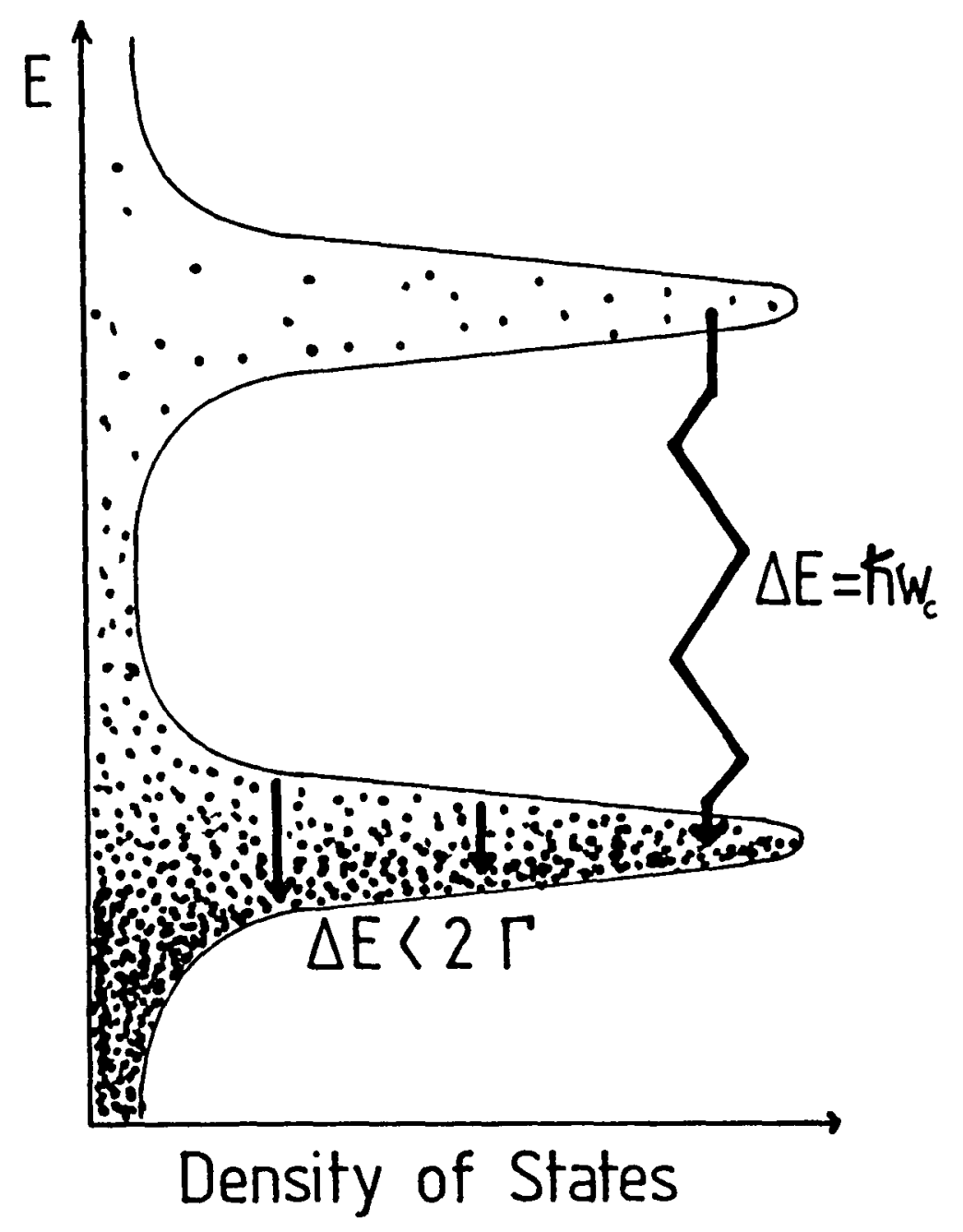

Figure 8.7 Schematic diagram of the distribution of electrons showing intra- and inter-Landau level transitions.

\subsection{CYCLOTRON PHONON EMISSION}

The following explanation is offered:-

In a magnetic field phonon emission may either be within a Landau level or between levels, Fig. 8.7, and these two processes will have very different temperature dependences.

For low electric fields there will not be a large population of electrons in Landau levels above the Fermi energy, so only the intra-Landau level scattering will take place. This should be similar to the process in zero field except for a factor from the enhanced densities of states in $2 \mathrm{D}$.

Once there is a significant population of a higher Landau level, energy relaxation may be by inter-Landau level scattering. This will occur at the fixed energy $\hbar \omega_{c}$ due to the emission of cyclotron phonons which were predicted by Slater \& Tremblay (1984); discussed by Slater (1985) and Toombs et al. (1987) and have been observed by Kent et al. (1987 \& 1988). The sharp peak in the densities of states at the centres of the levels will make this a very efficient process resulting in a rapid 
increase in the energy loss rate and effectively preventing electrons moving any higher in energy space.

The onset of cyclotron phonon emission, and hence the numerical value of $\lambda$, will depend on the distributions of electrons and phonons. Consideration of the statistics governing the available transitions shows that this will not vary greatly with the position of the Fermi level relative to the Landau levels. Challis et al. (1987) find the phonon distribution peaked at $\sim 4 \mathrm{kT}_{\mathrm{e}}$, but the exact value of the constant will vary with the temperature dependence of the phonon scattering and the measured $\lambda$ would need to be compared with a full theoretical calculation. The present situation, with a strong energy relaxation mechanism being activated once a certain electron temperature is reached, is analogous to the optic phonon emission found in the hot electron magnetophonon effect (reviews in Chapter 5, Barker 1978, Nicholas 1985), where it was found that the electron-electron scattering rate was not sufficient to maintain a Maxwellian carrier distribution. This would mean that the electron temperature is not terribly well defined and that a diffusion picture, as discussed by Yamada (1973), might give a better description.

It is interesting to consider why $\lambda$ is larger in G215 (the sample with a significant upper subband population), showing that cyclotron emission starts at lower electron temperatures. In Section 6.6 inter-subband scattering was shown to be acoustic phonon mediated, which means that the combination of an inter-subband transition with emission of a cyclotron phonon may bring a reduction in the threshold energy.

It is not particularly surprising that this sort of behaviour has not been seen before. In GaAs the cyclotron energy increases by $\sim 20 \mathrm{~K} / \mathrm{T}$, so to satisfy Eqn. 8.2 any measurements made at $2 \mathrm{~T}$ would require carrier temperatures in excess of $20 \mathrm{~K}$. This is about the upper limit of Hirakawa \& Sakaki's work, much hotter than all the other reported magnetoresistance measurements, and by these temperatures optic phonon emission is starting to dominate the situation (Das Sarma et al. 1988). However, it is intresting to note that Hollering et al. (1988) found an enhanced cooling rate which increased with magnetic field for $\mathrm{B}>8 \mathrm{~T}$, with carriers at 
temperatures above $50 \mathrm{~K}$ (using these values $\hbar \omega_{c}=3 \mathrm{kT}$ ), which they attributed to acoustic phonon emission. Also, Barlow et al. (1988) found an increased energy loss rate with $\mathrm{T}_{\mathrm{e}}>10 \mathrm{~K}$ at fields $\sim 2 \mathrm{~T}$ (exact value unspecified), which might be explained by cyclotron phonon emission.

A similar anomaly has recently been found by Blom et al. (1989) who used the amplitudes of Shubnikov-deHaas oscillations to deduce effective mass values from the Ando formula (Eqn. 8.1). They found that whenever $\mathrm{kT} / \hbar \omega_{\mathrm{c}}>0.3$ the values of $\mathrm{m}^{*}$ were smaller than those measured in cyclotron resonance. This appears to be the same result as obtained here and was also interpreted in terms of inter-Landau level scattering.

\subsection{INTRA-LANDAU LEVEL ENERGY LOSS}

The low electric field results, which obey the same temperature dependence as all those in higher magnetic fields, will now be discussed. Price (1982) predicts a $\mathrm{T}^{5}$ dependence for screened piezoelectric coupled acoustic phonon scattering at low temperatures, changing to $\mathrm{T}^{7}$ for deformation potential coupling at $\sim 2.5 \mathrm{~K}$ and eventually becoming linear at some unspecified high temperature. Other experimental results over the same temperature range show a similar behaviour to our data: those of Manion et al. agree almost exactly with those of Sample G156 i.e. $\dot{\mathrm{Q}} \propto \mathrm{T}^{3}$; while Hirakawa \& Sakaki see approx. $\mathrm{T}^{2}$; in GaInAs-InP systems under similar conditions Barlow et al. find an exponent between $3 \& 5$. There would appear to be three possible explainations for a $T^{3}$ dependence:-

(i) The temperature regime is somewhere between the low temperature ('Bloch-Gruneisen') and high temperature (equipartition) limits.

(ii) The major interaction is by unscreened piezoelectric coupling (Barlow et al. 1988, Price 1982).

(iii) There is an additional factor from the densities of states in magnetic field which needs to be included in the calculations.

The first is always a possibility which can only be properly discussed in the light of 
full numerical calculations. Hirakawa \& Sakaki have performed such a calculation and find a good fit to their data with the loss rate dominated by deformation potential acoustic phonon scattering. Ma et al. (1989) have performed a similar experiment and claim that piezoelectric and deformation potential coupling are of comperable importance, so a simple power law would not be expected at these temperatures.

In the second case we would also expect to see unscreened deformation potential scattering at moderate to higher temperatures, which would be associated with a $\mathrm{T}^{5}$ behaviour. However, considering the large densities of states found in Landau levels it is unlikely that any interaction should be unscreened.

Finally, it should be pointed out that all the calculations have been performed in the absence of a magnetic field. For Lorentzian broadened Landau levels the density of states $\triangle E$ away from the centre of a level is reduced by a factor of $1 / \Delta \mathrm{E}^{2}$. This leads to reduction of the transition rate like $q^{2}$, which leads to a reduction of $\mathrm{T}^{2}$ in the temperature dependence, but does not necessarily imply an overall reduction in the scattering rate because of the enhanced densities of states at the centres of the levels. Gammel et al. (1988) investigated the $p=5 / 2$ minimum in a field of $5 \mathrm{~T}$ and found an energy loss rate enhanced over the zero-field prediction (Price 1982), but following the same temperature dependence (Fig. 8.1). Their electron temperatures were $<33 \mathrm{mK}$, so all the excitations would be around the centre of the Landau level and may not see the effect of reduced densities of states in the wings of the levels.

Unfortunately, measurement of energy loss rates from Shubnikov-deHaas amplitudes can not be extended to sufficiently low magnetic fields for the transition to $B=0$ to be followed, as the oscillations will be unresolved outside the high field limit.

We now turn to a quantitative discussion of the scattering rates. By considering the energy balance expressed in Eqn. 8.3 a value for the energy relaxation rate for the intra-Landau level process, $\tau_{\epsilon}^{-1}$ may be deduced. These are typically $3 \times 10^{8} \mathrm{~s}^{-1} \mathrm{~K}^{-1}$ and are given for each sample in Table 8.2.

The loss rate $(\varphi)$ from Sample G156 agrees with that of Manion et al. who studied a sample of comparable carrier density but with a mobility a factor of 100 
lower, while those of Samples G104 and G215 are in agreement with Hirakawa \& Sakaki, who find no variation with $\mathrm{n}_{\mathrm{s}}$ or $\mu$. It is thus necessary to discuss how $\dot{Q}$ and $\tau_{\epsilon}^{-1}$ may depend on mobility and electron density.

In a low mobility sample $\mu$ will be mainly determined by impurities, which will lead to momentum relaxation of the carriers, but not necessarily energy relaxation to the lattice. It is thus quite possible that the d.c. mobility will not directly effect the electron energy loss rates. Further, all the energy transfer will only involve electrons within $\sim \mathrm{kT}$ of the Fermi energy and, within a given Landau level, this will be determined by the magnetic field and level broadening, but will be relatively insensitive to $\mathrm{n}_{s}$. This broadening depends on the single particle relaxation time, $\tau_{s}$, discussed in Chapter 7 , where it was shown that $\tau_{s}$ varied much less between samples than the transport mobility. It would be interesting to know how much $\tau_{s}$ varied between the samples studied by Hirakawa \& Sakaki, but it is probably fairly constant. Thus although some enhancement of the loss rate may be expected in high-mobility samples, these results do not support a very large enhacement and show no systematic variation with mobility.

Notice that the relaxation rates $\left(\tau \epsilon^{-1}\right)$ deduced for both the single subband samples are similar, while that of Sample G215 is greater. This may suggest that inter-subband scattering is important for energy loss or simply that the two subbands provide parallel channels for energy loss.

\subsection{SUMMARY}

In this chapter the decay in amplitude of Shubnikov-deHaas oscillations at elevated electron temperatures has been used to study electron energy loss rates at quite small magnetic fields. A strong field dependence is found, in contrast to earlier reports, which shows a greatly enhanced loss rate in the region $\mathrm{kT}_{e} \sim \hbar \omega_{c}$, which has been interpreted as evidence for cyclotron phonon emission. At lower electron temperatures or higher magnetic fields the energy loss rate is found to be proportional to $T_{e}{ }^{3}-T_{L}^{3}$ and reasons for this are explored. 


\section{REFERENCES}

Abstreiter G. and K. Ploog; Phys. Rev. Lett. 42, 1308 (1979).

Abstreiter G., R. Merlin and A. Pinczuk; J. Quant. Electron. 22, 1771 (1986).

Adams E.N. and T.D. Holstein; J. Phys. Chem. Solids 10, 254 (1959).

Ahrenkiel R.K. and F.C. Brown; Phys. Rev. A 136273 (1964).

Ando T.; J. Phys. Soc. Japan 37, 622 (1974a).

Ando T.; J. Phys. Soc. Japan 37, 1233 (1974b).

Ando T.; Solid State Commun. 21, 133 (1977).

Ando T.; J. Phys. Soc. Japan 51, 3893 (1982a).

Ando T.; J. Phys. Soc. Japan 51, 3900 (1982b).

Ando T., A.B. Fowler and F.Stern; Rev. Mod. Phys. 54, 437 (1982).

Ando T. and Y. Uemura; J. Phys. Soc. Japan 35, 1456 (1973).

Ando T. and Y. Uemura; J. Phys. Soc. Japan 36, 959 (1974).

Aoki H.; Rep. Prog. Phys. 50, 655 (1987).

Argyres P.N. and L.M. Roth; J. Phys. Chem. Solids 12, 89 (1959).

Barlow M.J., B.K.Ridley, M.J.Kane \& S.J.Bass; Solid State Electron. 31, 501 (1988).

Barker J.R.; Phys. Lett. 33A, 516 (1970).

Barker J.R.; J. Phys. C. 5, 1657 (1972).

Barker J.R. and C.J. McSheehy; Phys. Lett. 46A, 237 (1973).

Barker J.R.; Solid State Elect. 21, 197 (1978).

Barnes D.J., R.J. Nicholas, C.J.G.M. Langerak, J. Singleton, J.A.A.J. Perenboom, J.J. Harris and C.T. Foxon; to be published (1989).

Bass S.J. and M.L. Young; J. Crystal Growth 68, 311 (1984).

Bastard G.; Appl. Phys. Lett. 43, 591 (1983a).

Bastard G.; Acta Electronica 25, 147 (1983b).

Beinvogl W., Avid Kamgar and J.F. Koch; Phys. Rev. B 14, 4274 (1976).

Blakemore J.S. and J.A. Kennewell; J. Phys. C 8, 647 (1975).

Blom F.A.P., P.F. Fontein, J.H. Wolter, F.M. Peeters, X. Wu, F. Geerinckx and J.T. Devreese; Proc. EP2DS8 Grenoble to appear in Surf. Sci. (1989).

Braun M. and U. Rössler; J. Phys. C 18, 3365 (1985). 
Brodsky M. and G. Lucovsky; Phys. Rev. Lett. 21, 990 (1968).

Brummell M.A., R.J. Nicholas, J.C. Portal, K.Y. Cheng and A.Y. Cho; J. Phys. C 16, L579 (1983).

Brummell M.A., R.J. Nicholas, L.C. Brunel, S. Haunt, M.Baj, J.C.Portal, M.Razeghi, M.A. Di Forte-Poisson, K.Y. Cheng and A.Y. Cho; Surf. Sci. 142, 380 (1984).

Brummell M.A., R.J. Nicholas, M.A. Hopkins, J.J. Harris and C.T. Foxon; Phys. Rev. Lett. 58, 77 (1987a).

Brummell M.A., R.J. Nicholas, M.A. Hopkins, D.R. Leadley, J.J. Harris and C.T. Foxon; Phys. Rev. Lett. 59, 2820 (1987b).

Brummell M.A., D.R. Leadley, R.J. Nicholas, J.J. Harris and C.T. Foxon; Surf. Sci. 196, 451 (1988a)

Brummell M.A., D.R. Leadley, M.A. Hopkins and R.J. Nicholas; Physica Scripta T23, 73 (1988b).

Brunel L.C., S. Huant, R.J. Nicholas, M.A. Hopkins, M.A. Brummell, K. Karrai, J.C. Portal, M. Razeghi, K.Y. Cheng and A.Y. Cho; Surf. Sci. 170, 542 (1986).

Cantrell D.G. and P.N. Butcher; J. Phys. C 18, 5111 (1985).

Challis L.J., G.A. Toombs and F.W. Sheard; In Physics of Phonons (Ed. T. Paszhiewicz) Springer, 348 (1987).

Chang T.Y. and T.J. Bridges; Opt. Commun. 1, 423 (1970).

Cho A.Y. and J.R. Arthur; In Progress in Solid State Chemistry

(Ed. J.O. McCaldin and G. Somorjai), Pergamon, Oxford. 10, 157 (1976).

Coleridge P.T., R. Stoner and R. Fletcher; Phys. Rev. B 39, 1120 (1989).

Das Sarma S.; Phys. Rev. B 27, 2590 (1983).

Das Sarma S.; Phys. Rev. Lett. 52, 859 (1984).

Das Sarma S. and B.A.Mason; Phys. Rev. B 31, 5536 (1985).

Das Sarma S. and F. Stern; Phys. Rev. B 32, 8442 (1985).

Das Sarma S., J.K. Jain and R. Jalabert; Phys. Rev. B 37, 6290 (1988).

Das Sarma S. and X.C. Xie; Phys. Rev. Lett. 61, 738 (1988).

Devresse J.T. (Ed.); Polarons in Ionic Crystals, North Holland, Amsterdam (1972).

Devresse J.T. and F.M. Peeters (Eds.); Polarons and Excitons in Polar Semiconductors and Ionic Crystals, Plenum, New York (1984).

Dingle R.B.; Proc. Royal Soc. A211, 517 (1952).

Dingle R., H.L. Störmer, A.C. Gossard and W.Wiegmann; Appl. Phys. Lett. 33, 665 (1978).

Dolgopolov V.T., A.A. Shashkin, S.I. Dorozhkin and E.A. Vryodov; Sov. Phys. JETP 62, 1219 (1985). 
Dolling G. and J.L.T. Waugh; In Lattice Dynamics (Ed. R.F. Wallis)

Pergamon Press, Oxford. 19 (1965).

Dorozhkin S.I., F. Lell and W. Schoepe; Solid State Commun. 60, 245 (1986).

Drummond T.J., J. Klein, D. Arnold, R. Fisher, R.E. Thorne, W.G. Lyons and H. Morkoç; Appl. Pyhs. Lett. 42, 615 (1983).

Eaves L., R.A.Stradling, S.Askenazy, R.Barbaste, G.Carrère, J.Leotin, J.C.Portal and P.Ulmet; J. Phys. C 7, 1999 (1974).

Eaves L., R.A.Hoult, R.A.Stradling, R.J. Tidey, J.C. Portal and S.Askenazy; J. Phys. C 8, 1034 (1975).

Eaves L., R.A.Hoult, R.A.Stradling, S.Askenazy, R.Barbaste, G.Carrere, J.Leotin, J.C.Portal and P.Ulmet; J. Phys. C 10, 2831 (1977).

Eaves L. and J.C. Portal; J. Phys. C 12, 2809 (1979).

Eisenstein J.P., H.L. Stormer, V. Narayanamurti, A.Y. Cho, A.C. Gossard and C.W. Tu; Phys. Rev. Lett. 55, 875 (1985).

Englert Th., D.C. Tsui, A.C. Gossard and Ch. Uihlein; Surf. Sci. 113, 295 (1982a)

Englert Th., D.C. Tsui, J.C. Portal, J. Beerens and A.C. Gossard;

Solid State Commun. 44, 1301 (1982b).

Englert Th., J.C.Maan, D.C.Tsui \& A.C.Gossard; Solid State Commun. 45, 989 (1983)

Fang F.F. and W.E. Howard; Phys. Rev. Lett. 16, 797 (1966).

Fang F.F. and P.J. Stiles; Phys. Rev. 174, 823 (1968).

Fang F.F., T.P. Smith III and S.L. Wright; Surf. Sci. 196, 310 (1988).

Fletcher R., E. Zaremba, M. D'Iorio, C.T. Foxon and J.J. Harris;

Phys. Rev. B 38, 7861 (1988).

Fowler A.B., F.F. Fang, W.E.Howard and P.J.Stiles; Phys. Rev. Lett. 16, 901 (1966).

Foxon C.T. and J.J. Harris; Philips J. Res. 41, 313 (1986).

Foxon C.T., J.J. Harris, R.G. Wheeler and D.E. Lacklison;

J. Vac. Sci. Technol. B4, 511 (1986).

Foxon C.T. and J.J. Harris; Private Communication (1989).

Frölich H.; Adv. Phys. 3, 325 (1954).

Fujisawa I.; Jap. J. Appl. Phys. 17, 667 (1978).

Gammel P.L., D.J. Bishop, J.P. Eisenstein, J.H. English, A.C. Gossard, R. Ruel and H.L. Stormer; Phys. Rev. B 38, 10128 (1988).

Gauthier D., L. Dmowski, J.C. Portal, D.R. Leadley, M.A. Hopkins, M.A.Brummell, R.J. Nicholas, M.Razeghi and P. Maurel; Superlatt. \& Microstruct. 4, 201 (1988).

Gavrilov M.G. and I.V. Kukushkin; JETP Lett. 43, 79 (1986). 
Gerhadts R.R.; Z. Physik B21, 275 (1975).

Gerhadts R.R. and V. Gudmundsson; Phys. Rev. B 34, 2999 (1986).

Goetz K.H., D. Bimberg, H. Jurgenson, F. Selders, A.V. Sotomonov, G.F. Glinskii and M. Razeghi; J. Appl. Phys. 54, 4543 (1983).

Gornik E., R. Lassnig, G. Stasser, H.L. Störmer, A.C. Gossard and W. Wiegmann; Phys. Rev. Lett. 54, 1820 (1985).

Grégoris G.; PhD Thesis, Toulouse (1985).

Grégoris G., J. Beerens, S. Ben Amor, L. Dmowski, J.C. Portal, F. Alexandre, D.L. Sivco and A.Y. Cho; Phys. Rev. B 37, 1262 (1988).

Grimes C.C.; Surf. Sci. 73, 379 (1978).

Gudmundsson V. and R.R. Gerhardts; Phys. Rev. B 35, 8005 (1987).

Gurevich V.L. and Y.A. Firsov; Sov. Phys. JETP 13, 137 (1961).

Hamaguchi C., S. Mori, H. Murata and K. Taniguchi; Springer Solid State Science 87, 541 (1988).

Harper P.G., J.W. Hodby and R.A. Stradling; Rep. Prog. Phys. 36, 1 (1973).

Harrang J.P., R.J. Higgins, R.K. Goodall, P.R. Jay, M. Laviron and P. Delescluse; Phys. Rev. B 32, 8126 (1985).

Harris J.J., C.T. Foxon, D.E. Lacklison and K.W.J. Barnham; Superlattice \& Microstructures 2, 563 (1986).

Harris J.J., D.E. Lacklison, C.T. Foxon, F.M. Selton, A.M. Suckling, R.J. Nicholas and K.W.J. Barnham; Semicond. Sci. Technol. 2, 783 (1987).

Harris J.J., J.M. Lagemaat, S.J. Battersby, C.M. Hellon, C.T. Foxon and D.E. Lacklison; Semicond. Sci. Technol. 3, 773 (1988).

Herrmann R. and C. Weisbuch; Phys. Rev. B 15, 823 (1977).

Herrmann R., W.Kraak, G.Nachtwei and Th.Schurig; Phys. Stat. Sol. b129 415 (1985).

Hess K. and P. Vogl; Solid State Commun. 30, 807 (1979).

Hirakawa K. and H. Sakaki; Appl. Phys. Lett. 49, 889 (1986).

Hjalmarson H.P. and T.J. Drummond; Appl. Phys. Lett. 48, 656 (1986).

Hollering R.W.J., T.T.J.M. Berendschhot, H.J.A. Bluyssen, H.A.J.M. Reinen, P. Wyder and F. Roozeboom; Phys. Rev. B 38, 13323 (1988).

Höpfel R.A. and G. Weimann; Appl. Phys. Lett. 46, 291 (1985).

Hopkins M.A., R.J. Nicholas, M.A. Brummell, J.J. Harris and C.T. Foxon; Superlattices \& Microstuctures 2, 319 (1986).

Hopkins M.A.; D.Phil thesis, Oxford (1987). 
Hopkins M.A., R.J. Nicholas, M.A. Brummell, J.J. Harris and C.T. Foxon; Phys. Rev. B 36, 4789 (1987).

Hopkins M.A., R.J. Nicholas, D.J. Barnes, M.A. Brummell, J.J. Harris and C.T. Foxon; Phys. Rev. B 39, (1989).

Horst M., U.Merkt and J.P. Kotthaus; Phys. Rev. Lett. 50, 754 (1983).

Horst M., U.Merkt, W.Zawadzki, J.C.Mann and K.Ploog; Solid State Commun. 53, 403 (1985).

Hoult R.A.; D.Phil Thesis, Oxford (1974).

Howell D.F.; D.Phil thesis, Oxford (1989).

Hudson P.A.; Proc. 4th Int. Con Magnet Technology, Brookhaven, (USAEC Conf-720904) 444 (1972).

Hudson P.A. and H. Jones; IEEE Trans. Magn. 17, 1649 (1981).

Hudson P.A., H. Jones and H.M. Whitworth; Journal de Physique 45, 55 (1984)

Inoue, M., H. Hida, M. Inayama, Y. Inuishi, K. Nanbu and S. Hiyamizu; Physica 117b, 720 (1983).

Isihara A. and L. Smrčka; J. Phys. C 19, 6777 (1986).

Janak J.F.; Phys. Rev. 178, 1416 (1969).

Jones, H. and H.M. Whitworth; IEEE Trans. Magn. 22, 443 (1986).

Joyce, B.A.; Rep. Prog. Phys. 48, 1637 (1985).

Kahlert H. and G. Bauer; Phys. Stat. Sol. b46, 535 (1971).

Kane E.O.; J. Phys. Chem. Solids 1, 249 (1957).

Kane M.J., D.A. Anderson, L.L.Taylor and S.J. Bass; J. Appl. Phys. 60, 657 (1986).

Kane M.J., D.R. Leadley and D. Gauthier; Private communication.

Karrai K., S.Huant, G.Martinez and L.C.Brunel; Solid State Commun. 66355 (1988).

Kent A.J., V.W. Rampton, M.I. Newton, P.J.A. Carter, G.A. Hardy, P. Hawker, P.A. Russell and L.J. Challis; Surf. Sci. 196, 410 (1988).

Kido G., N. Miura, H. Ohno and H. Sakaki; J. Phys. Soc. Japan 51, 2168 (1982).

Kido G. and N. Miura; J. Phys. Soc. Jap. 52, 1734 (1983).

Kim O.K. and W.G. Spitzer; J. Appl. Phys. 50, 4362 (1979).

Klitzing K. von, G. Dorda and M. Pepper; Phys. Rev. Lett. 45494 (1980).

Knight, D.J.E.; "Ordered List of FIR Lines" NPL Report QU45 (1981).

Kubo R.; J. Phys. Soc. Japan 12, 570 (1957). 
Kubo R., S.J. Miyake and N. Hashitsume; Solid State Phys. 17, 279 (1965).

Lacklison D.E,, Harris J.J., C.T. Foxon, J. Hewett, D. Hilton and C. Roberts;

Semicond. Sci. Technol. 3, 633 (1988).

Lang D.V. and R.A. Logan; Phys. Rev. Lett. 39, 635 (1977).

Lang D.V., R.A. Logan and M. Jaros; Phys. Rev. B 19, 1015 (1979).

Langerak C.J.G.M., J. Singleton, P.J.van der Wel, J.A.A.J.Perenboom, D.J. Barnes, R.J. Nicholas, M.A. Hopkins and C.T. Foxon; Phys. Rev. B 38, 13133 (1988).

Larsen D.M.; In Polarons in Ionic Crystals and Polar Semiconductors

(Ed. J.T. Devreese) North Holland (1972).

Larsen D.M.; Phys. Rev. B 30, 4595 (1984).

Lassnig R. and W. Zawadzki; J. Phys. C 16, 5435 (1983).

Lassnig R. and W. Zawadzki; Surf. Sci. 142, 361 (1984a).

Lassnig R. and W. Zawadzki; Surf. Sci. 142, 388 (1984b).

Lassnig R.; Surf. Sci. 170, 549 (1986).

Leadley D.R., R.J. Nicholas, L.L. Taylor, S.J. Bass and M.S. Skolnick; Springer Solid State Science 87, 545 (1988a).

Leadley D.R., M.A. Brummell, R.J. Nicholas, J.J. Harris and C.T. Foxon; Solid State Electron. 31, 781 (1988b).

Leadley D.R., R.J. Nicholas, J.J. Harris and C.T. Foxon; Semicond. Sci. Technol. 4 (1989a).

Leadley D.R., R.J. Nicholas, J.J. Harris and C.T. Foxon; Semicond. Sci. Technol. 4 (1989b).

Leadley D.R., R.J. Nicholas, J.J. Harris and C.T. Foxon; Proc. $6^{\text {th }}$ Int. Conf. on Hot Carriers in Semiconductors, (1989c) to appear in Solid State Electronics.

Linde D. von der, J. Kuhl and H. Klingenberg; Phys. Rev. Lett. 44, 1505 (1980).

Lindemann G., R.Lassnig, W.Seidenbusch and E.Gornik; Phys. Rev. B 284693 (1983).

Ma Y., R. Fletcher, E. Zaremba, M. D'Iorio, C.T. Foxon and J.J. Harris;

Proc. EP2DS8 Grenoble to appear in Surf. Sci. (1989).

Maciel A.C., L.C. Campelo Cruz and J.F. Ryan; J. Phys. C 20, 3041 (1987).

Mani R.G. and J.R. Anderson; Phys. Rev. B 37, 4299 (1988).

Manion S.J., M. Artaki, M.A. Emanuel, J.J. Coleman and K. Hess; Phys. Rev. B 35, 9203 (1987).

Maude D.K., J.C. Portal, L. Dmowski, T. Foster, L. Eaves, M.Nathan, M. Heiblum, J.J. Harris and R.B. Beall; Phys. Rev. Lett. 59, 815 (1987).

Mears A.L., R.A. Stradling and E.K. Inall; J. Phys. C 1, 821 (1968). 
Mears A.L. and R.A. Stradling; J. Phys. C 4, L22 (1971).

Meseguer F., J.C. Maan and K. Ploog; Phys. Rev. B 35, 2505 (1987).

Mooradian A. and G.B. Wright; Solid State Commun. 4, 431 (1966).

Mori S. and T. Ando; J. Phys. Soc. Japan 48, 865 (1980).

Mori S., K. Taniguchi, C. Hamaguchi, S. Sasa and S. Hiyamizu; J. Phys. C 21, 1791 (1988a).

Mori S., H. Murata, K. Taniguchi and C. Hamaguchi; Phys. Rev. B 38, 7622 (1988b).

Mowbray D.J., W. Hayes, J.A.C. Bland, M.S. Skolnik and S.J. Bass;

Semicond. Sci. Technol. 2, 822 (1987).

Nash K.J., M.S. Skolnick and S.J. Bass; Semicond. Sci. Technol. 2, 329 (1987)

Nasir F., J. Singleton and R.J. Nicholas; Semicond. Sci. Technol. 3, 654 (1988).

Nelson R.J.; Appl. Phys. Lett. 31, 351 (1977).

Neugebauer T. and G. Landwehr; Phys. Rev. B 21, 702 (1980).

Newson D.J., K-F. Beerggren, M. Pepper, H.W. Myron, G.J. Davies and E.G. Scott; J. Phys. C 19, L403 (1986).

Nicholas R.J. and R.A. Stradling; J. Phys. C 9, 1253 (1976).

Nicholas R.J. and R.A. Stradling; J. Phys. C 12, 2829 (1979).

Nicholas R.J., R.A.Stradling, J.C.Portal and S.Askenazy; J. Phys. C 12, 1653 (1979).

Nicholas R.J., S.J. Sessions and J.C. Portal; Appl. Phys. Lett. 37, 178 (1980).

Nicholas R.J., L.C. Brunel and J.C. Portal; Surf. Sci. 113, 290 (1982).

Nicholas R.J.; Prog. Quant. Elect. 10, 1 (1985).

Nicholas R.J., L.C.Brunel, S.Huant, K.Karrai, J.C.Portal, M.A.Brummell, M.Razeghi, K.Y.Cheng and A.Y.Cho; Phys. Rev. Lett. 55, 883 (1985).

Nicholas R.J., M.A. Brummell, M.A. Hopkins and D.R. Leadley; Plenum Physics Series B 179, 243 (1987).

Nicholas R.J., R.J.Haug, K.von Klitzing \& G.Weimann; Phys. Rev. B 37, 1294 (1988).

Nicholas R.J., D.J. Barnes, D.R. Leadley, C.J.G.M. Langerak, J. Singleton, P.J. van der Wel, J.A.A.J. Perenboom, J.J. Harris and C.T. Foxon; Proc. NATO A.S.I., Venice (1989a).

Nicholas R.J., S. Ben Amor, J.C. Portal, D.L. Sivco and A.Y. Cho; Semicond. Sci. Technol. 4, 116 (1989b).

Nicholas R.J. and H. Sigg; to be published.

Ougezzaden A., R. Mellet, H. Thibierge, Y. Gao and A. Mircea; Proc. $3^{\text {rd }}$ Euro. Workshop on MOVPE, Montpelleir, (1989). 
Paalanen M.A., D.C. Tsui and J.C.M. Hwang; Phys. Rev. Lett. 51, 2226 (1983).

Palik E.D., G.S. Picus, S. Teitler and R.F. Wallis; Phys. Rev. 122, 475 (1961).

Pearsall T.P., R. Carles and J.C. Portal; Appl. Phys. Lett. 42, 436 (1983).

Peierls R.E.; Ann. Phys., Lpz. 10, 97 (1931).

Peeters F.M. and J.T. Devreese; Phys. Rev. B 23, 1936 (1981).

Peeters F.M. and J.T. Devreese; Phys. Rev. B 31, 3689 (1985).

Peeters F.M., X. Wu and J.T. Devreese; Physica Scripta T13, 282 (1986a).

Peeters F.M., X. Wu and J.T. Devreese; Phys. Rev. B 33, 4338 (1986b).

Peeters F.M., X. Wu and J.T. Devreese; Phys. Rev. B 36, 7518 (1987).

Peeters F.M., X. Wu and J.T. Devreese; Surf. Sci. 196, 437 (1988).

Peeters F.M., P. Warmenbol and J.T. Devreese; Preprint (1989).

Perterson R.L.; In Semiconductors and Semimetals

(Eds. R.K. Willardson and A.C. Beer) 10, 221 (1975).

Pickering C.J.; Electron. Mater. 10, 901 (1981).

Pinczuk A., J.M. Worlock, H.L. Stormer, R. Dingle, W. Wiegmann and A.C. Gossard; Solid State Commun. 36, 43 (1980).

Ploog K. and M.A. Brummell; Private Communication (1988).

Portal J.C., R.J. Nicholas, E. Kress-Rogers, A. Chevy, J. Besson, J. Galibert and P. Perrier; J. Phys. Soc. Japan 49, Suppl. A, 879 (1980)

Portal J.C., R.J. Nicholas, M.A. Brummell, A.Y. Cho, K.Y. Chang and T.P. Pearsall; Solid State Commun. 43, 907 (1982)

Portal J.C., J. Cisowski, R.J. Nicholas, M.A. Brummell, M. Razeghi and M.A. Poisson; J. Phys. C 16, L573 (1983).

Portal J.C., G. Gréorgis, M.A. Brummell, R.J. Nicholas, M. Razeghi, M.A. Di Forte-Poisson, K.Y. Cheng and A.Y. Cho; Surf. Sci. 142, 368 (1984).

Price P.J.; J. Appl. Phys. 53, 6863 (1982).

Puri S.M. and T.H. Geballe; Bull. Am. Phys. Soc 8, 309 (1963).

Ramage J.C., R.J.Nicholas, R.A.Stradling and F.Kuchar; J. Phys. C 10, L611 (1977).

Razeghi M., M.A. Poisson, J.P. Larivain, B. deCemoux, J.P. Duchemin and M. Voos; Electron. Lett. 43, 293 (1982).

Razeghi M., J.P. Hirtz, U.D. Ziemelis, C. Delalande, B. Etienne and M. Voos; Appl. Phys. Lett. 43, 585 (1983). 
Razeghi M., J.P. Duchemin, J.C. Portal, L. Dmowski, G. Remeni, R.J. Nicholas and A. Briggs; Appl. Phys. Lett. 48, 712 (1986).

Rikken G.L.J.A., H. Sigg, C.J.G.M. Langerak, H.W. Myron, P.A.A.J. Perenboom and G. Weimann; Phys. Rev. B 34, 5590 (1986).

Rode D.L.; In Semiconductors and Semimetals

(Ed. R.K. Willardson \& A.C. Beer) 10, 1 (1975).

Roukes M.L., M.R. Freeman, R.S. Germain, R.C. Richardson and M.B. Ketchen; Phys. Rev. Lett. 55, 422 (1985).

Sakaki H., K. Hirakawa, J. Yoshino, S.P. Svensson, Y. Sekiguchi, T. Hotta, S. Nishii and N. Miura; Surf. Sci. 142, 306 (1984).

Sakaki H., T. Noda, K. Hirakawa, M. Tanaka and T. Matsusue; Appl. Phys. Lett. 51, 1934 (1987).

Sallen R.P. and E.L. Key; IRE Trans. on Circuit Theory 2, 74 (1955).

Sarkar C.K. and R.J. Nicholas; Surf. Sci. 113, 326 (1982).

Sarkar C.K., R.J. Nicholas, J.C. Portal, M. Razeghi, J. Chevrier and J. Massies; J. Phys. C 18, 2667 (1985).

Sawaki N.; Surf. Sci. 170, 537 (1986).

Schiff L.I.; "Quantum Mechanics" McGraw-Hill, New York, pp37-42 (1968).

Seeger K.; "Semiconductor Physics" Springer-Verlag 117 (1973).

Seidenbusch W., B.Lindemann, R.Lassnig, J.Edlinger and E.Gornik; Surf. Sci. 142, 375 (1984).

Senda K., K. Shimomae, K. Kasai and C. Hamaguchi; J. Phys. Jap. 47, 551 (1979).

Shah J., R.F.Leheny, R.E.Nahory and M.A.Pollack; Appl. Phys. Lett. 37, 475 (1980).

Shah J., A.Pincuzuk, A.C.Gossard and W.Wiegmann; Appl. Phys. Lett. 42, 55 (1983).

Shah J., A.Pincuzuk, A.C.Gossard and W.Wiegmann; Phys. Rev. Lett. 54, 2045 (1985)

Shah J.; IEEE J. Quant. Electron. 22, 1728 (1986).

Shubnikov L. and W.J. de Haas; K. Akad. Amsterdam, Proc. 33, 4, 363 (1930).

Sigg H., P.Wyder and J.A.A.J.Perenboom; Phys. Rev. B 31, 5253 (1985).

Singleton J., R.J.Nicholas and F.Nasir; Solid State Commun. 58, 883 (1986a).

Singleton J., R.J. Nicholas, F. Nasir and C.K. Sarkar; J. Phys. C 19, 35 (1986b).

Singleton J., R.J. Nicholas, D.C. Rogers and C.T. Foxon; Surf. Sci. 196, 429 (1988).

Skolnick M.S., P.R. Tapster, S.J. Bass, N. Apsley, A.D. Pitt, N.G. Chew,

A.G. Cullis, S.P. Aldred and C.A. Warwick; Appl. Phys. Lett. 48, 1455 (1986a). 
Skolnick M.S., P.R. Tapster, S.J. Bass, A.D. Pitt, N. Apsley and S.P. Aldred; Semicond. Sci. Technol. 1, 29 (1986b).

Skolnick M.S., K.J. Nash, P.R. Tapster, D.J. Mowbray, S.J. Bass and A.D. Pitt; Phys. Rev. B 35, 5925 (1987).

Skolnick M.S., K.J. Nash, S.J. Bass, P.E. Simmonds and M.J. Kane; Solid State Commun. 67, 637 (1988).

Slater G.W. and A.M.S. Tremblay; Phys. Rev. B 29, 2289 (1984).

Slater G.W.; J. Phys. C 18, 2483 (1985).

Smith D.T.; D.Phil. thesis, Oxford (1967).

Smith R.A.; Semiconductors p104ff C.U.P. (1978).

Smith T.P., B.B.Goldberg, P.J. Stiles and M. Heiblum; Phys. Rev. B 32, 2696 (1985).

Smith T.P., W.I. Wang and P.J. Stiles; Phys. Rev. B 34, 2995 (1986).

Smith T.P. III and F.F. Fang; Phys. Rev. B 37, 4303 (1988).

Smith T.P. III, F.F.Fang, U.Meirav and M. Heiblum; Phys. Rev. B 38, 12744 (1988).

Stahl E., D.Weiss, G.Weimann, K.vonKlitzing and K.Ploog; J. Phys. C 18, 783 (1985)

Stein D., K. von Klitzing and G. Weimann; Phys. Rev. Lett. 51, 130 (1983).

Stern, F.; Phys. Rev. B 5, 4891 (1972).

Stern F. and W.E. Howard; Phys. Rev. 163, 816 (1967).

Stern F. and S. Das Sarma; Phys. Rev. B 30, 840 (1984).

Stinchcombe R.B.; Proc. Phys. Soc. 78, 275 (1961).

Stinchcombe R.B.; In Transport Phenomena (Ed. G. Kircenow \& J. Marro), Springer Lecture Notes in Physics 31, 368 (1974).

Störmer H.L., R. Dingle, A.C. Gossard, W. Wiegmann and M.D. Sturge; Solid State Commun. 29, 705 (1979).

Störmer H.L., A.C. Gossard and W. Wiegmann; Solid State Commun. 41, 707 (1982).

Stradling R.A. and R.A. Wood; J. Phys. C 1, 1711 (1968).

Stradling R.A. and R.A. Wood; J. Phys. C 3, L94 (1970a).

Stradling R.A. and R.A. Wood; J. Phys. C 3, 2425 (1970b).

Swierkowski L., W. Zawadzki, Y. Guldner and C. Rigaux; Solid State Commun. 27, 1245 (1978).

Thornber K.K. and R.P. Feynman; Phys. Rev. B 1, 4099 (1970).

Toombs G.A., F.W. Sheard, D. Neilson and L.J. Challis; Solid State Commun. 64, 577 (1987). 
Tsui D.C.; Solid State Commun. 9, 1789 (1971).

Tsui D.C., Th. Englert, A.Y.Cho and A.C. Gossard; Phys. Rev. Lett. 44, 341 (1980).

Usher A.; D.Phil Thesis, Oxford (1988).

Van Houten H., J.G. Williamson, M.E.I. Broekaart, C.T. Foxon and J.J. Harris; Phys. Rev. B 37, 2756 (1988).

Van Royen J., J. De Sitter and J.T. Devreese; Phys. Rev. B 30, 7154 (1984).

Vass E.; Solid State Commun. 61, 127 (1987).

Walukiewicz W., H.E.Ruda, J.Lagowski and H.C.Gatos; Phys. Rev. B 30, 4571 (1984).

Warmenbol P., F.M. Peeters and J.T. Devreese; Phys. Rev. B 37, 4694 (1988).

Warmenbol P., F.M. Peeters and J.T. Devreese; Phys. Rev. B 39, 7821 (1989).

Warmenbol P.; Proc. NATO A.S.I., Venice (1989).

Wei H.P., A.M. Chang, D.C. Tsui and M. Razeghi; Phys. Rev. B 32, 7016 (1985).

Weiss D, K. von Klitzing and V. Mosser; Springer Solid State Science 67, 204 (1986).

Wendler L. and R. Haupt; Phys. Stat. Sol. b1 43, 487 (1987).

Wennberg A.K.M., S.N. Ytterboe, C.M. Gould, H.M. Bozler, J. Kelm and H. Morkoc; Phys. Rev. B 34, 4409 (1986).

Wood R.A., R.A. Stradling and I.P. Molodyan; J. Phys. C 3, L154 (1970).

Wu X., F.M. Peeters and J.T. Devreese; Phys. Rev. B 32, 6982 (1985).

Wu X., F.M. Peeters and J.T. Devreese; Phys. Rev. B 34, 8800 (1986).

Wu X., F.M. Peeters and J.T. Devreese; Phys. Stat. Sol. b143, 581 (1987).

Yamada E.; Solid State Commun. 13, 503 (1973).

Yamada E. and T. Kurosawa; J. Phys. Soc. Japan 34, 603 (1973).

Yang C.H., J.M. Carlson-Swindle, S.A. Lyon and J.M. Worlock; Phys. Rev. Lett. 55, 2359 (1985).

Yuasa T. and M. Ishii; Phys. Rev. B 37, 7001 (1988).

Zawadzki W. and P. Pfeffer; Springer Solid State Science 71, 523 (1986).

Ziesmann M., D. Heitmann and L.L. Chang; Phys. Rev. B 35, 4541 (1987).

Zhao W.Q., J.F. Koch, J. Ziegler and H. Maier; Phys. Rev. B 31, 2416 (1985). 